\title{
An Aliphatic Bischler-Napieralski reaction: Dihydropyridones by \\ Cyclocarbonylation of 3-Allylimidazolidin-4-ones
}

Mostafa M. Amer, Olatz Olaizola, Jennifer Carter, Hossay Abas, and Jonathan Clayden*

\section{Supporting Information}

\section{Contents}

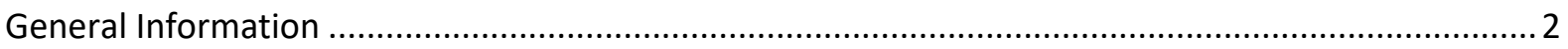

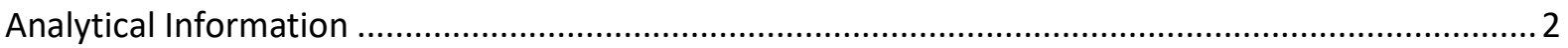

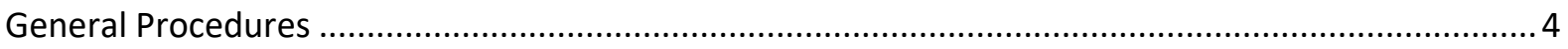

Optimisation of alkylation of trans $\mathrm{N}$-chloroformylimidazolidinone ................................................. 6

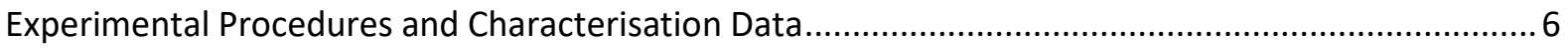

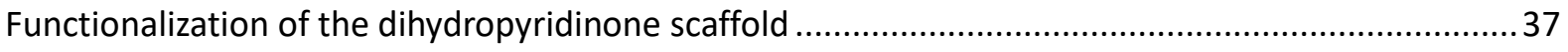

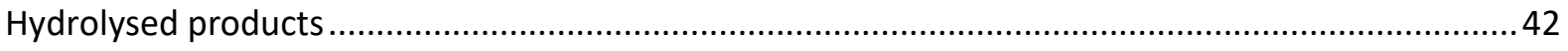

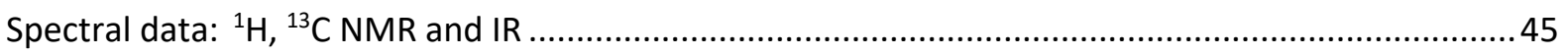

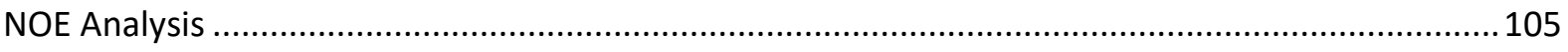

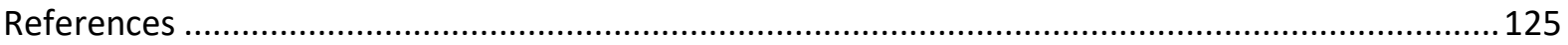




\section{General Information}

Where specified, reactions requiring anhydrous conditions were executed under dry nitrogen atmospheres in glassware that was dried using either a combination of vacuum and heat-gun, oven, or flame drying. Reaction mixtures were stirred magnetically. Reaction carried out in a microwave reactor were completed on a Biotage Initiator+. Air- and moisture-sensitive liquids and solutions were transferred via syringe into the reaction vessels through rubber septa. All reagents were purchased at highest commercial quality and used as received (unless specified otherwise). Solvents were purchased at the highest commercial quality and used as received (unless specified otherwise). Anhydrous $\mathrm{DCM}, \mathrm{Et}_{2} \mathrm{O}, \mathrm{THF}$ and $\mathrm{MeCN}$ were collected under argon from an Anhydrous Engineering alumina-column drying system. Anhydrous pyridine stored over $3 \AA ̊$ molecular sieves was purchased from Acros. All reaction temperatures described below $-10{ }^{\circ} \mathrm{C}$ were achieved using acetone/dry ice cooling baths.

\section{Analytical Information}

$\mathbf{R}_{\boldsymbol{f}}$ : TLC was performed on aluminium backed silica plates $\left(0.2 \mathrm{~mm}, 60 \mathrm{~F}_{254}\right)$ which were developed using standard visualising agent: UV fluorescence $(254 \& 366 \mathrm{~nm})$, potassium permanganate stain.

Chromatography: Flash chromatography was performed on an automated Biotage Isolera ${ }^{\mathrm{TM}}$ Spektra Four using gradient elution on pre-packed silica gel Biotage ${ }^{\circledR}$ SNAP Ultra columns.

mp: Melting points $(\mathrm{mp})$, expressed in ${ }^{\circ} \mathrm{C}$, were measured on a Kofler hotstage melting point apparatus and are uncorrected.

IR: IR spectra were recorded from neat compounds applied as thin films on a Perkin Elmer (Spectrum One) FT-IR spectrometer (ATR sampling accessory). Only absorbances of interest ( $v_{\max }$ expressed in $\mathrm{cm}^{-1}$ ) are reported.

NMR: Spectra were recorded on Varian VNMR $(400 \mathrm{MHz}$ or $500 \mathrm{MHz}$ ) instruments, and Bruker (400 $\mathrm{MHz}$ or $500 \mathrm{MHz}$ ). Chemical shifts $(\delta)$ are quoted in parts per million (ppm) downfield of trimethylsilane. Spectra were calibrated using the residual solvent peaks for $\mathrm{CDCl}_{3}\left(\delta_{H}: 7.26 \mathrm{ppm} ; \delta_{\mathrm{C}}\right.$ : $77.16 \mathrm{ppm})$ or $\mathrm{D}_{2} \mathrm{O}\left(\delta_{\mathrm{H}}: 4.79 \mathrm{ppm}\right)$ as appropriate. Coupling constants $(J)$ are quoted in $\mathrm{Hz}$ and are rounded to the nearest $0.1 \mathrm{~Hz}$. Splitting patterns are abbreviated to: singlet (s), doublet (d), triplet $(t)$, quartet (q), quintet (quin.), multiplet (m), broad (br.) or some combination thereof. 2D NMR experiments COSY, HSQC and HMBC were used in assigning NMR spectra where necessary. 
MS: Low resolution mass spectra were recorded by the technical staff at the University of Bristol on a Bruker Daltronics MicrOTOF 2 mass spectrometer (ESI), with only molecular ions of interest ([M]+, $[\mathrm{M}+\mathrm{H}]^{+},[\mathrm{M}+\mathrm{Na}]^{+}$) reported.

HRMS: High resolution mass spectra were recorded by the technical staff at the University of Bristol on a Bruker Daltronics MicrOTOF 2 mass spectrometer (ESI).

Optical Rotations: $\left([\alpha]_{\mathbb{D}}^{\mathrm{T}}\right)$ Optical rotations were measured on a Bellingham and Stanley Ltd. ADP220 polarimeter using a cell with a pathlength of $0.25 \mathrm{dm}$ with the solvent quoted and concentration $(c)$ given in $\mathrm{g} / 100 \mathrm{~mL}$.

X-Ray Sample Preparation: Crystals suitable for X-ray were grown by dissolving the sample in a dense solvent (DCM), in which it is reasonably soluble, which is then placed inside a second chamber containing a less dense solvent (Hexane or $n$-Pentane). The solvents slowly mixed together, altering the polarity of the solution in which the sample is dissolved, leading to crystallisation (Figure 1). The resulting crystals are isolated by filtration washing with ice cold DCM, before drying under high vacuum.

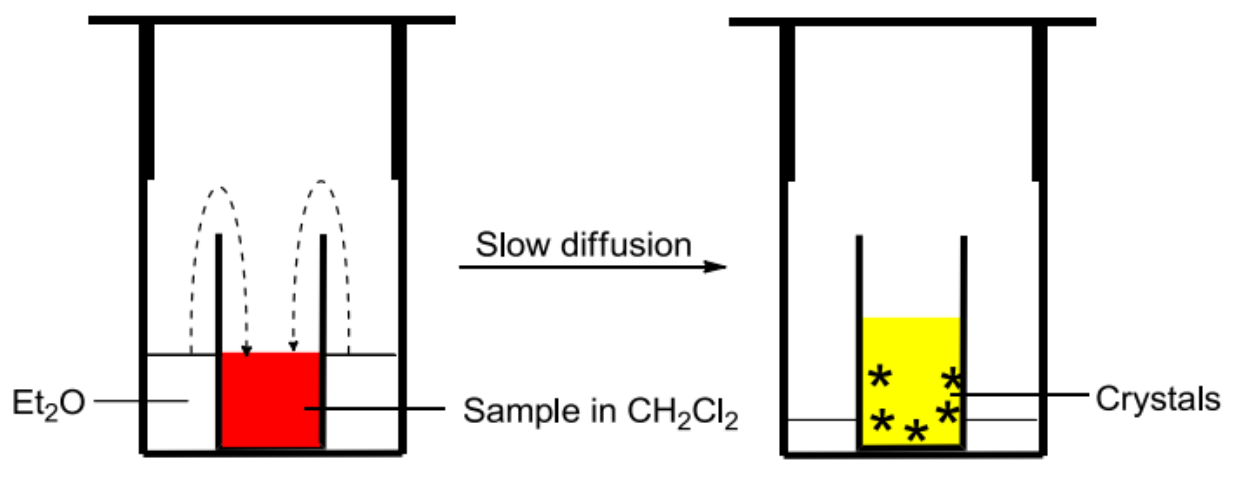

Figure 1: Crystallisation by slow diffusion. 


\section{General Procedures}

General Procedure 1 (GP1): Alkylation of trans N-chloroformylimidazolidinone

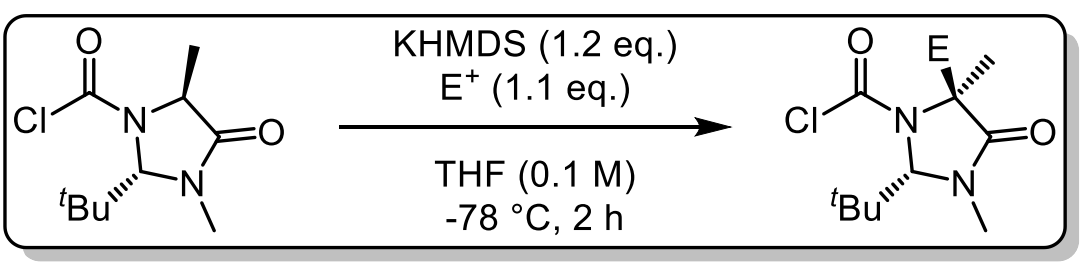

(2S,5S)-2-(Tert-butyl)-3,5-dimethyl-4-oxoimidazolidine-1-carbonyl chloride 1a (1.0 eq.) was dissolved in dry THF (0.1 M) under a nitrogen atmosphere and the solution was cooled to $-78{ }^{\circ} \mathrm{C}$. KHMDS $(1.0$ $\mathrm{M}$ in THF, 1.1 to 1.2 eq.) was added dropwise the reaction was left to stir for 2 min at $-78{ }^{\circ} \mathrm{C}$. The electrophile (1.1 eq.) was added and the reaction mixture was left to stir at $-78{ }^{\circ} \mathrm{C}$ for $2 \mathrm{~h}$. The reaction mixture was quenched with aqueous $\mathrm{HCl}(1.0 \mathrm{M})$. After warming up to room temperature, the reaction mixture was partitioned between $\mathrm{H}_{2} \mathrm{O}$ and EtOAc. The aqueous phase was further extracted with EtOAc $(\mathrm{x} 2)$ and the combined organic phases were washed with brine, dried over $\mathrm{MgSO}_{4}$, filtered and the solvent was removed under reduced pressure. The crude product was purified by flash column chromatography to yield the desired alkylated product.

General Procedure 2 (GP2): Lactamisation of alkylated trans N-chloroformylimidazolidinone utilising 2,6-lutidine

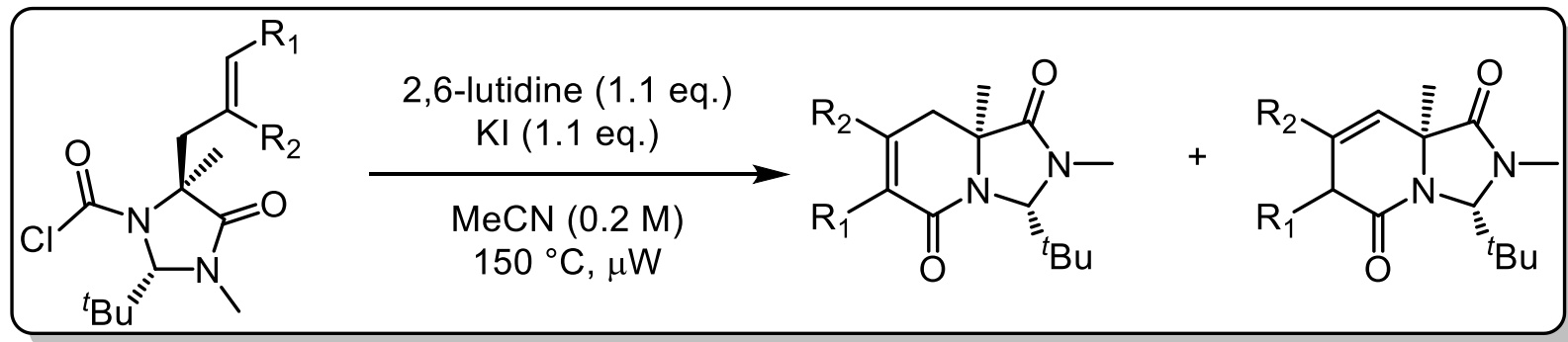

$\mathrm{N}$-Chloroformylimidazolidinone (1.0 eq.) was dissolved in dry $\operatorname{MeCN}(0.2 \mathrm{M})$ in a microwave vial before the addition of potassium iodide (1.1 eq.) and 2,6-lutidine (1.1 eq.). The sealed vial was heated in a microwave reactor to $150{ }^{\circ} \mathrm{C}$ for $2 \mathrm{~h}$. The reaction mixture was quenched with aqueous $\mathrm{HCl}(1.0 \mathrm{M})$. The reaction mixture was partitioned between $\mathrm{H}_{2} \mathrm{O}$ and EtOAc. The aqueous phase was further extracted with EtOAc (x2) and the combined organic phases were washed with brine, dried over $\mathrm{MgSO}_{4}$, filtered and the solvent was removed under reduced pressure. The crude product was purified by flash column chromatography to yield the desired lactam. 
General Procedure 3 (GP3): Lactamisation of alkylated trans $N$-chloroformylimidazolidinone utilising DBU

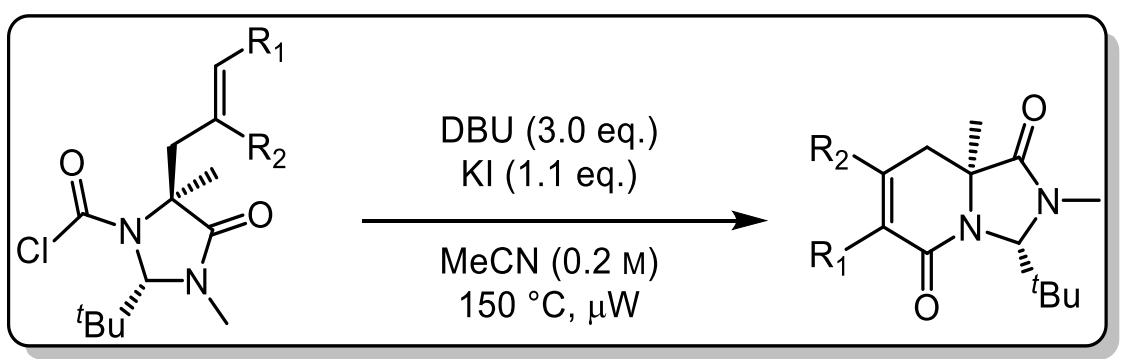

$\mathrm{N}$-Chloroformylimidazolidinone (1 eq.) was dissolved in dry $\operatorname{MeCN}(0.2 \mathrm{M})$ in a microwave vial before the addition of potassium iodide (1.1 eq.) and DBU (3.0 eq.). The sealed vial was heated in a microwave reactor to $150{ }^{\circ} \mathrm{C}$ for $2 \mathrm{~h}$. The reaction mixture was quenched with aqueous $\mathrm{HCl}(1.0 \mathrm{M})$. The reaction mixture was partitioned between $\mathrm{H}_{2} \mathrm{O}$ and EtOAc. The aqueous phase was further extracted with EtOAc (x2) and the combined organic phases were washed with brine, dried over $\mathrm{MgSO}_{4}$, filtered and the solvent was removed under reduced pressure. The crude product was purified by flash column chromatography to yield the desired lactam.

General Procedure 4 (GP4): Isomerisation of unconjugated isomer 4 to conjugated isomer 3.

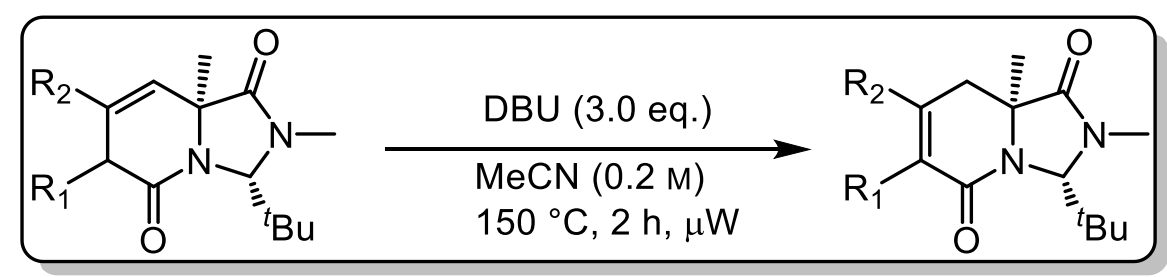

The unconjugated isomer 4 (1 eq.) was dissolved in dry $\mathrm{MeCN}(0.2 \mathrm{M})$ in a microwave vial before the addition of DBU (3.0 eq.). The sealed vial was heated in a microwave reactor to $150{ }^{\circ} \mathrm{C}$ for $2 \mathrm{~h}$. The reaction mixture was quenched with aqueous $\mathrm{HCl}(1.0 \mathrm{M})$. The reaction mixture was partitioned between $\mathrm{H}_{2} \mathrm{O}$ and EtOAc. The aqueous phase was further extracted with EtOAc $(\times 2)$ and the combined organic phases were washed with brine, dried over $\mathrm{MgSO}_{4}$, filtered and the solvent was removed under reduced pressure. The crude product was purified by flash column chromatography to yield the desired lactam $\mathbf{3}$. 


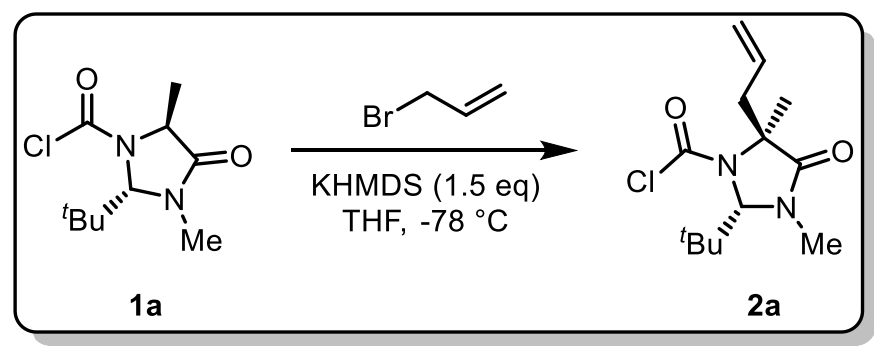

\begin{tabular}{l|ll}
\multicolumn{1}{l}{ Entry } & Addition of electrophile & Yield/ \% \\
\hline $\mathbf{1}$ & 10 mins after KHMDS & 37 \\
$\mathbf{2}$ & Before KHMDS & - \\
$\mathbf{3}$ & 5 mins after KHMDS & 49 \\
$\mathbf{4}^{*}$ & 5 mins after KHMDS & 65 \\
$\mathbf{5}^{*}$ & 2 mins after KHMDS & 82
\end{tabular}

\section{Experimental Procedures and Characterisation Data}

Synthesis of (2S,5S)-2-(tert-butyl)-3,5-dimethyl-4-oxoimidazolidine-1-carbonyl chloride 1a Step 1:

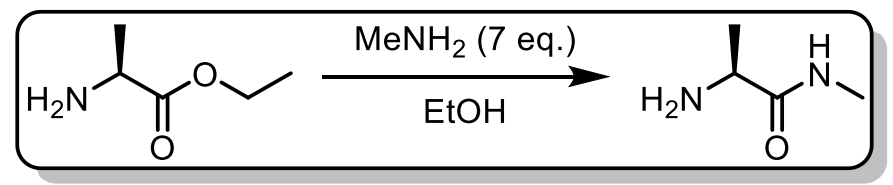

(S)-2-Amino-N-methylpropanamide S1

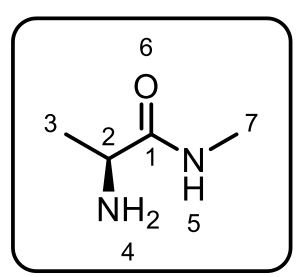

L-Alanine ethyl ester hydrochloride $(8.00 \mathrm{~g}, 52.1 \mathrm{mmol}, 1.0 \mathrm{eq}$.) was dissolved in $\mathrm{MeNH}_{2}$ solution ( $33 \%$ in $\mathrm{EtOH}, 45.4 \mathrm{~mL}, 365 \mathrm{mmol}, 7.0$ eq.) and the reaction mixture stirred at RT for $48 \mathrm{~h}$. The solvent was removed under reduced pressure and the solid residue dissolved in $\mathrm{CHCl}_{3}(100 \mathrm{~mL})$ and washed with aqueous $\mathrm{NaHCO}_{3}$ (sat. aq.) (15 ml). The aqueous layer was extracted with $\mathrm{CHCl}_{3}$ $(3 \times 100 \mathrm{~mL})$. The combined organic phases were washed with brine, dried over $\mathrm{MgSO}_{4}$, filtered and the solvent was removed under reduced pressure to yield the title compound $(2.48 \mathrm{~g}, 47 \%)$ as a yellow oil, which was subsequently used without further purification. ${ }^{1} \mathrm{H}$ NMR (400 $\left.\mathrm{MHz}, \mathrm{CDCl}_{3}\right) \delta 3.48$ (q, J= $7.0 \mathrm{~Hz}, 1 \mathrm{H}, \mathrm{H}-2$ ), 2.80 (d, J=5.0 Hz, 3H, H-3), 1.48 (s, 2H, H-4), 1.32 (d, J = 29.2, 3H, H-7). ${ }^{13} \mathrm{C}$ NMR (101 MHz, CDCl 3 ) $\delta 176.27$ (C-1), 50.77 (C-2), 25.79 (C-7), 21.82 (C-3). Data in agreement with reported values. ${ }^{1}$ 
Step 2:

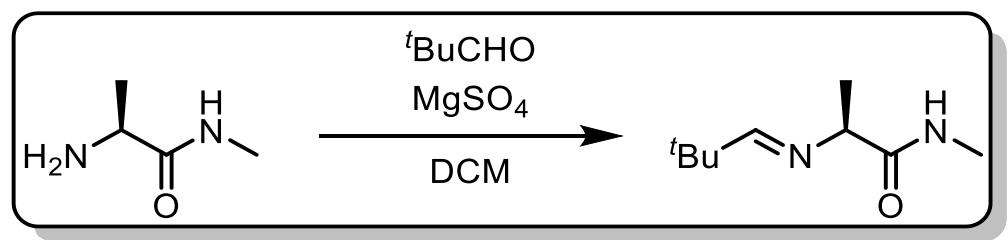

(S)-2-((2,2-Dimethylpropylidene)amino)- $N$-methylpropanamide S2

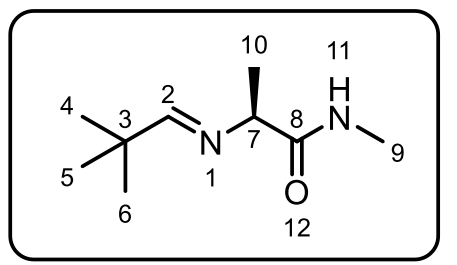

To a solution of $\mathrm{N}$-methylamide $\mathbf{S 1}(2.48 \mathrm{~g}, 24.3 \mathrm{mmol}, 1.0$ eq.) in dry $\mathrm{DCM}(16.2 \mathrm{~mL}, 1.0 \mathrm{M})$ was added $\mathrm{MgSO}_{4}(2.92 \mathrm{~g}, 24.3 \mathrm{mmol}, 1.0$ eq.) followed by pivaldehyde (3.93 mL, $36.4 \mathrm{mmol}, 1.5$ eq.) and the reaction mixture was left to stir at $\mathrm{RT}$ for $16 \mathrm{~h}$. The reaction mixture was filtered and concentrated under reduced pressure to afford the title compound (3.97 $\mathrm{g}, 96 \%)$ as a yellow oil which was subsequently used without further purification. ${ }^{1} \mathrm{H}$ NMR $\left(400 \mathrm{MHz}, \mathrm{CDCl}_{3}\right) \delta 7.50(\mathrm{~s}, 1 \mathrm{H}, \mathrm{H}-2), 6.90(\mathrm{~s}, 1 \mathrm{H}, \mathrm{H}-11), 3.66(\mathrm{q}, J=7.0 \mathrm{~Hz}, 1 \mathrm{H}$, $\mathrm{H}-7$ ), 2.82 (d, J = 5.0 Hz, 3H, H-9), 1.29 (d, J = 7.0 Hz, 3H, H-10), 1.05 (s, 9H, H-4 + H-5 + H-6); ${ }^{13} \mathrm{C} \mathrm{NMR}$ (126 MHz; CDCl 8174.78 (C-2), 173.14 (C-8), 67.58 (C-7), 36.51 (C-3), 26.79 (C-4 + C-5 + C-6), 25.96 (C-9), 21.46 (C-10). Data in agreement with reported values. ${ }^{1}$

Step 3:

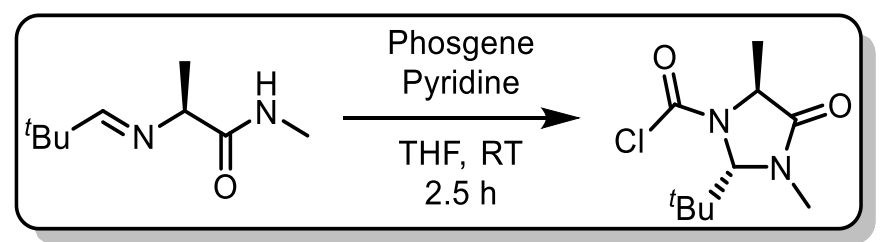

(2S,5S)-2-(Tert-butyl)-3,5-dimethyl-4-oxoimidazolidine-1-carbonyl chloride 1a

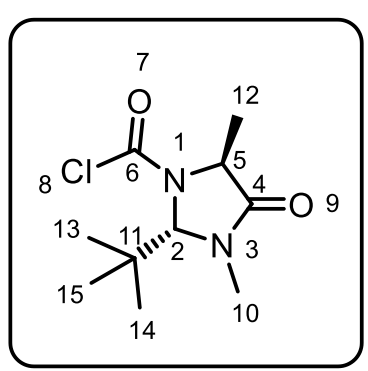

Imine S2 (3.97 g, $23.3 \mathrm{mmol}, 1.0$ eq.) was dissolved in dry THF ( $35 \mathrm{~mL}, 0.65$ $\mathrm{M})$, and phosgene solution (15\% wt. in toluene, $26.7 \mathrm{~mL}, 35.0 \mathrm{mmol}, 1.5$ eq.) was added dropwise over $30 \mathrm{~min}$ (an off-white suspension was observed after $5 \mathrm{~min}$ ). The reaction mixture was stirred at RT for $2.5 \mathrm{~h}$. To the reaction mixture, pyridine $(3.76 \mathrm{~mL}, 46.6 \mathrm{mmol}, 2.0$ eq.) was added and the reaction was left to stir for $16 \mathrm{~h}$. The reaction mixture was quenched with aqueous $\mathrm{HCl}(1.0 \mathrm{M})$. The reaction mixture was partitioned between $\mathrm{H}_{2} \mathrm{O}$ and EtOAc. The aqueous phase was further extracted with EtOAc ( $\mathrm{x} 2$ ) and the combined organic phases were washed with brine ( $\mathrm{x} 2)$, dried over $\mathrm{MgSO}_{4}$, filtered and the solvent was removed under reduced pressure. The crude product was chromatographed $\left(\mathrm{SiO}_{2}\right.$, pet.ether/EtOAc, 5:1) and the title compound was isolated as a mixture of diastereomers (97:3 trans:cis diastereomeric ratio by ${ }^{1} \mathrm{H}$ NMR). The product was further recrystallised from $\mathrm{EtO}_{2} /$ pet. ether to afford the title trans compound $(3.80 \mathrm{~g}, 70 \%)$ as a pale yellow solid. $\mathbf{R}_{f}=0.32$ (pet.ether/EtOAc $\left.=5: 1\right) ;[\alpha]_{\mathrm{D}}^{23}=-24\left(c=0.5\right.$ in $\left.\mathrm{CHCl}_{3}\right) ; \mathbf{I R} \mathbf{v}_{\max }=2968,2934(\mathrm{C}-\mathrm{H})$, 1745, $1709(\mathrm{C}=0) ;{ }^{1} \mathbf{H}$ NMR $\left(400 \mathrm{MHz}, \mathrm{CDCl}_{3}\right.$ ) (mixture of rotamers $\mathrm{A}: \mathrm{B}$ in a 0.42:0.58 ratio) $\delta 5.12$ (s, $1 \mathrm{H}, \mathrm{H}-5$, rot. A), $5.10(\mathrm{~s}, 1 \mathrm{H}, \mathrm{H}-5$, rot. B), 4.10 (br s, $1 \mathrm{H}, \mathrm{H}-2$, rot. $\mathrm{A}+\operatorname{rot} . \mathrm{B}), 3.01$ (br s, $3 \mathrm{H}, \mathrm{H}-10$, rot. A + rot. $B), 1.70(\mathrm{~s}, 3 \mathrm{H}, \mathrm{H}-12$, rot. A), $1.56(\mathrm{~d}, 3 \mathrm{H}, \mathrm{H}-12, \mathrm{~J}=5.2 \mathrm{~Hz}$, rot. B), $1.02(\mathrm{~s}, 9 \mathrm{H}, \mathrm{H}-13+\mathrm{H}-14+\mathrm{H}-$ 15, rot. B), 0.97 (s, $9 \mathrm{H}, \mathrm{H}-13+\mathrm{H}-14+\mathrm{H}-15$, rot. A); $\left.{ }^{13} \mathrm{C} \mathrm{NMR} \mathrm{(126} \mathrm{MHz,} \mathrm{CDCl}\right) \delta 171.30$ (C-4, rot. B), 171.06 (C-4, rot. A), 147.70 (C-6, rot. A), 145.36 (C-6, rot. B), 83.88 (C-5, rot. A), 82.72 (C-5, rot. B), 58.52 (C-2, rot. B), 57.46 (C-2, rot. A), 41.50 (C-11, rot. A), 41.21 (C-11, rot. A), 32.35 (C-10, rot. A + rot. B), 26.85 (C-13 + C-14 + C-15, rot. B), 26.45 (C-13 + C-14 + C-15, rot. A), 19.82 (C-12, rot. A), 15.98 (C-12, rot. B). Data in agreement with reported values. ${ }^{1}$ 


\section{Alkylated $\mathbf{N}$-chloroformylimidazolidinones}

\section{(2S,5S)-5-Allyl-2-(tert-butyl)-3,5-dimethyl-4-oxoimidazolidine-1-carbonyl chloride 2a}
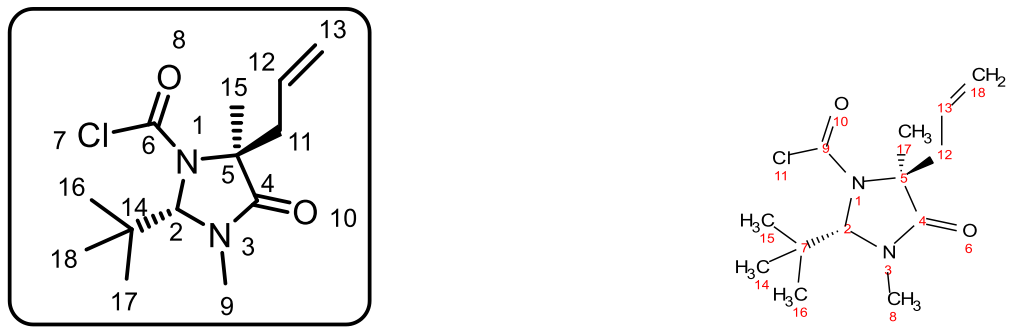

Following GP1, trans $N$-chloroformylimidazolidinone $1 \mathrm{a}$ (510 mg, $2.19 \mathrm{mmol}, 1.0$ eq.) was dissolved in dry THF $(22 \mathrm{~mL}, 0.1 \mathrm{M})$ and the solution was cooled to $-78{ }^{\circ} \mathrm{C}$ before the dropwise addition of KHMDS ( $2.62 \mathrm{ml}, 1.0 \mathrm{M}$ in THF, 1.2 eq.). The reaction was left to stir for $2 \mathrm{~min}$ at $-78^{\circ} \mathrm{C}$. Allyl bromide $\left(0.208 \mathrm{ml}, 2.40 \mathrm{mmol}, 1.1 \mathrm{eq}\right.$.) was added and the reaction mixture stirred at $-78{ }^{\circ} \mathrm{C}$ for $2 \mathrm{~h}$. The reaction mixture was quenched with aqueous $\mathrm{HCl}(1.0 \mathrm{M})$ and extracted with EtOAc $(\times 2)$. The combined organic layers were washed with brine, dried over $\mathrm{MgSO}_{4}$, filtered and the solvent was removed under reduced pressure. The crude material was purified by flash column chromatography $\left(\mathrm{SiO}_{2}\right.$, pet.ether/EtOAc, 7:3) to afford the title compound ( $488 \mathrm{mg}, 82 \%$ ) as a pale yellow oil. $\mathbf{R}_{f}=0.28$ (pet.ether/EtOAc $=7: 3) ;[\alpha]_{\mathrm{D}}^{23}=-72\left(c=1\right.$ in $\left.\mathrm{CHCl}_{3}\right)$.

IR $\mathbf{V}_{\max }=2972,2901(\mathrm{C}-\mathrm{H}), 1740,1715(\mathrm{C}=\mathrm{O}) ;{ }^{1} \mathbf{H}$ NMR $\left(400 \mathrm{MHz}, \mathrm{CDCl}_{3}\right)$ (mixture of rotamers A: $\mathrm{B}$ in a 0.49:0.51 ratio) $\delta 5.46-5.29(\mathrm{~m}, 1 \mathrm{H}, \mathrm{H}-12$, rot. A + rot. B), $5.27-5.06(\mathrm{~m}, 3 \mathrm{H}, \mathrm{H}-13+\mathrm{H}-2$, rot. A + rot. B), 3.17 (ddd, $J=50.9,14.3,8.6 \mathrm{~Hz}, 1 \mathrm{H}, \mathrm{H}-11$, rot. B), 3.02 (d, J=4.6 Hz, 3H, H-9, rot. A + rot. B), 2.59 (ddd, $J=69.1,14.3,6.0 \mathrm{~Hz}, 1 \mathrm{H}, \mathrm{H}-11$, rot. A), 1.65 (d, $J=27.8 \mathrm{~Hz}, 3 \mathrm{H}, \mathrm{H}-15$, rot. A + rot. B), 1.05 (d, $J=34.3 \mathrm{~Hz}, 9 \mathrm{H}, \mathrm{H}-16+\mathrm{H}-17+\mathrm{H}-18$, rot. A + rot. B). ${ }^{13} \mathrm{C}$ NMR (101 MHz, CDCl 3 ) $\delta 172.26$ (C-4), 149.07 (C-6), 130.87 (C-12, rot. A), 130.53 (C-12, rot. B), 121.35 (C-13, rot. A), 121.13 (C-13, rot. B), 83.39 (C-2, rot. B), 83.04 (C-2, rot. A), 69.23 (C-5, rot. B), 66.92 (C-5, rot. A), 42.78 (C-11, rot. B), 39.89 (C-11, rot. A), 39.67 (C-14, rot. A), 39.23 (C-14, rot. B), 31.93 (C-9, rot. A), 31.58 (C-9, rot. B), 27.64 (C-16 + C-17 + C-18, rot. B), 26.97 (C-16 + C-17 + C-18, rot. A), 23.19 (C-15, rot. A), 22.46 (C-15, rot. B); HRMS $\mathrm{C}_{13} \mathrm{H}_{22} \mathrm{ClN}_{2} \mathrm{O}_{2}[\mathrm{M}+\mathrm{H}]^{+}$calculated: 273.1364 , found: 273.1375 . Data in agreement with reported values. ${ }^{2}$

\section{$(2 S, 5 S)-2$-(Tert-butyl)-3,5-dimethyl-4-oxo-5-((E)-3-(trimethylsilyl)allyl)imidazolidine-1-carbonyl chloride $2 \mathrm{k}$}
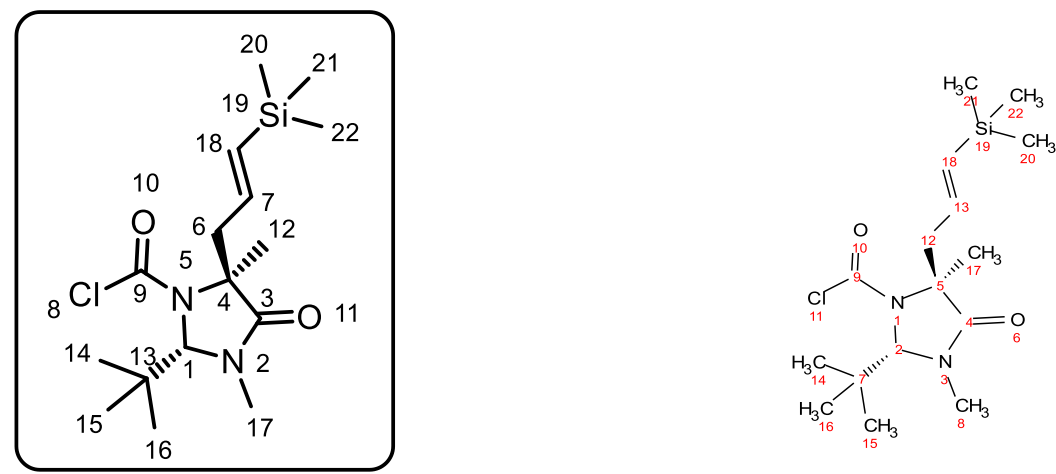
Following GP1, trans $\mathrm{N}$-chloroformylimidazolidinone $1 \mathrm{a}$ (100 $\mathrm{mg}, 0.430 \mathrm{mmol}, 1.0 \mathrm{eq}$.) was dissolved in dry THF (4.30 mL, $0.1 \mathrm{M})$ and the solution was cooled to $-78{ }^{\circ} \mathrm{C}$ before the dropwise addition of KHMDS (0.473 ml, 1.0 M in THF, 1.1 eq.). The reaction was left to stir for $2 \mathrm{~min}$ at $-78{ }^{\circ} \mathrm{C}$. (E)-(3Bromoprop-1-en-1-yl)trimethylsilane $(91.3 \mathrm{mg}, 0.473 \mathrm{mmol}, 1.1 \mathrm{eq}$.$) was added and the reaction$ mixture stirred at $-78{ }^{\circ} \mathrm{C}$ for $2 \mathrm{~h}$. The reaction mixture was quenched with aqueous $\mathrm{HCl}(1.0 \mathrm{M})$ and extracted with EtOAc (x2). The combined organic layers were washed with brine, dried over $\mathrm{MgSO}_{4}$, filtered and the solvent was removed under reduced pressure. The crude material was purified by flash column chromatography $\left(\mathrm{SiO}_{2}\right.$, pet.ether/EtOAc, 7:3) to afford the title compound $(93.3 \mathrm{mg}$, $63 \%$ ) as a pale yellow oil. $\mathbf{R}_{f}=0.51$ (pet.ether/EtOAc $\left.=7: 3\right) ;[\alpha]_{\mathrm{D}}^{23}=-32\left(c=1\right.$ in $\left.\mathrm{CHCl}_{3}\right)$.

IR (neat, cm ${ }^{-1}$ ): $v_{\max } 2958(\mathrm{C}-\mathrm{H}), 1743(\mathrm{C}=\mathrm{O}), 1715(\mathrm{C}=\mathrm{H}), 1699(\mathrm{C}=\mathrm{O}) ;{ }^{1} \mathbf{H}$ NMR (400 MHz, CDCl $)_{3}$ (mixture of rotamers A:B in a 0.45:0.55 ratio) $\delta 5.87(\mathrm{t}, J=17.7 \mathrm{~Hz}, 1 \mathrm{H}, \mathrm{H}-18), 5.65-5.49(\mathrm{~m}, 1 \mathrm{H}, \mathrm{H}-$ 7), 5.00 (d, $J=1.9 \mathrm{~Hz}, 1 \mathrm{H}, \mathrm{H}-1$ ), 3.19 (ddd, $J=54.8,13.9,8.4 \mathrm{~Hz}, 1 \mathrm{H}, \mathrm{H}-6), 3.00$ (d, $J=5.2 \mathrm{~Hz}, 3 \mathrm{H}, \mathrm{H}-$ 17), 2.63 (ddd, $J=61.5,14.1,5.7 \mathrm{~Hz}, 1 \mathrm{H}, \mathrm{H}-6), 1.64$ (d, J = 26.6 Hz, 3H, H-12), 1.04 (d, J = 34.2 Hz, 9H, $\mathrm{H}-14+\mathrm{H}-15+\mathrm{H}-16), 0.02(\mathrm{~d}, J=4.1 \mathrm{~Hz}, 9 \mathrm{H}, \mathrm{H}-20+\mathrm{H}-21+\mathrm{H}-22) ;{ }^{13} \mathrm{C} \mathrm{NMR}\left(101 \mathrm{MHz}, \mathrm{CDCl}_{3}\right) \delta 144.40$ (C-3, rot. A + rot. B), 138.67 (C-7, rot. B), 138.49 (C-7, rot. A), 138.10 (C-18, rot. B), 138.00 (C-18, rot. A), 83.63 (C-1, rot. B), 83.22 (C-1, rot. A), 70.37 (C-4, rot. A), 69.62 (C-4, rot. B), 45.28 (C-6, rot. A), 42.42 (C-6, rot. B), 39.69 (C-13, rot. A), 39.28 (C-13, rot. B), 31.97 (C-17, rot. A), 31.63 (C-17, rot. B), 27.58 (C-14 + C-15 + C-16, rot. B), 26.92 (C-14 + C-15 + C-16, rot. A), 23.17 (C-12, rot. A), 22.42 (C-12, rot. B), -1.15 (C-20 + C-21 + C-22, rot. B), $-1.29\left(\left(\mathrm{C}-20+\mathrm{C}-21+\mathrm{C}-22\right.\right.$, rot. A); HRMS $\mathrm{C}_{16} \mathrm{H}_{29} \mathrm{ClN}_{2} \mathrm{O}_{2} \mathrm{Si}[\mathrm{M}$ $+\mathrm{Na}]^{+}$calculated: 367.1579 , found: 367.1597 .

\section{(2S,5S)-2-(Tert-butyl)-3,5-dimethyl-4-oxo-5-(2-phenylallyl)imidazolidine-1-carbonyl chloride 2b}
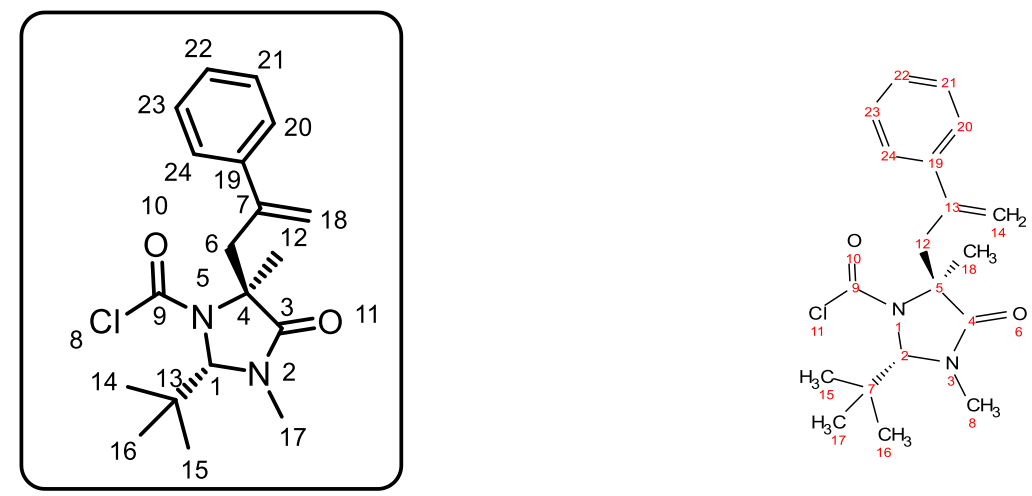

Following GP1, trans $N$-chloroformylimidazolidinone $1 \mathrm{a}(161 \mathrm{mg}, 0.692 \mathrm{mmol}, 1.0$ eq.) was dissolved in dry THF (6.92 mL, $0.1 \mathrm{M})$ and the solution was cooled to $-78^{\circ} \mathrm{C}$ before the dropwise addition of KHMDS (0.761 ml, $1.0 \mathrm{M}$ in THF, 1.1 eq.). The reaction was left to stir for $2 \mathrm{~min}$ at $-78{ }^{\circ} \mathrm{C}$. (3Bromoprop-1-en-2-yl)benzene (150 mg, $0.761 \mathrm{mmol}, 1.1$ eq.) was added and the reaction mixture stirred at $-78^{\circ} \mathrm{C}$ for $2 \mathrm{~h}$. The reaction mixture was quenched with aqueous $\mathrm{HCl}(1.0 \mathrm{M})$ and extracted with EtOAc (x2). The combined organic layers were washed with brine, dried over $\mathrm{MgSO}_{4}$, filtered and the solvent was removed under reduced pressure. The crude material was purified by flash column chromatography $\left(\mathrm{SiO}_{2}\right.$, pet.ether/EtOAc, 7:3) to afford the title compound (121.0 $\left.\mathrm{mg}, 50 \%\right)$ as a pale yellow oil. $\mathbf{R}_{f}=0.39$ (pet.ether/EtOAc $\left.=7: 3\right) ;[\alpha]_{\mathrm{D}}^{23}=-76\left(c=1\right.$ in $\left.\mathrm{CHCl}_{3}\right)$.

IR $v_{\max }=2968,2930(\mathrm{C}-\mathrm{H}), 1733,1701(\mathrm{C}=\mathrm{O}) ;{ }^{1} \mathrm{H}$ NMR $\left(400 \mathrm{MHz}, \mathrm{CDCl}_{3}\right)$ (mixture of rotamers A: $\mathrm{B}$ in a 0.46:0.54 ratio) $\delta 7.36-7.19(\mathrm{~m}, 5 \mathrm{H}, \mathrm{H}-20+\mathrm{H}-21+\mathrm{H}-22+\mathrm{H}-23+\mathrm{H}-24$, rot. $\mathrm{A}+\operatorname{rot} . \mathrm{B}), 5.32(\mathrm{~d}, J=$ 
$60.5 \mathrm{~Hz}, 1 \mathrm{H}, \mathrm{H}-18$, rot. A), 5.21 (t, $J=2.0 \mathrm{~Hz}, 1 \mathrm{H}, \mathrm{H}-18$, rot. B), 4.70 (d, $J=14.0 \mathrm{~Hz}, 1 \mathrm{H}, \mathrm{H}-1$, rot. A + rot. B), $3.41(t, J=1.1 \mathrm{~Hz}, 1 \mathrm{H}, \mathrm{H}-6$, rot. $B), 3.41-3.14(\mathrm{~m}, 1 \mathrm{H}, \mathrm{H}-6$, rot. $A), 2.11(\mathrm{~d}, J=22.4 \mathrm{~Hz}, 3 \mathrm{H}, \mathrm{H}-$ 17, rot. $A+\operatorname{rot} . B), 1.67(d, J=26.9 \mathrm{~Hz}, 3 \mathrm{H}, \mathrm{H}-12$, rot. $A+\operatorname{rot} . \mathrm{B}), 0.94(\mathrm{~d}, J=38.9 \mathrm{~Hz}, 9 \mathrm{H}, \mathrm{H}-14+\mathrm{H}-15$ + H-16, rot. A + rot. B); ${ }^{13} \mathrm{C}$ NMR (101 MHz, CDCl $) \delta 166.29$ (C-3), 148.83 (C-9, rot. A), 143.83 (C-9, rot. B), 128.29, 127.94, 127.75, 127.01, (C-7, C-20, C-21, C-22, C-23, C-24, rot. A + rot. B) 122.37 (C18, rot. B), 122.01 (C-18, rot. A), 82.87 (C-1, rot. A), 82.50 (C-1, rot. B), 68.03 (C-4, rot. A), 65.04 (C-4, rot. B), 44.34 (C-6, rot. B), 41.88 (C-6, rot. A), 39.67 (C-13, rot. A), 39.45 (C-13, rot. B), 31.00 (C-17, rot. B), 30.77 (C-17, rot. A), 27.71 (C-14 + C-16 + C-17, rot. A ), 27.03 (C-14 + C-16 + C-17, rot. B), 24.37 (C-12, rot. B), 23.29 (C-12, rot. A); HRMS $\mathrm{C}_{19} \mathrm{H}_{26} \mathrm{ClN}_{2} \mathrm{O}_{2}[\mathrm{M}+\mathrm{H}]^{+}$calculated: 349.1677 , found: 349.1673.

(2S,5S)-2-(Tert-butyl)-3,5-dimethyl-5-(2-(naphthalen-2-yl)allyl)-4-oxoimidazolidine-1-carbonyl chloride $2 f$
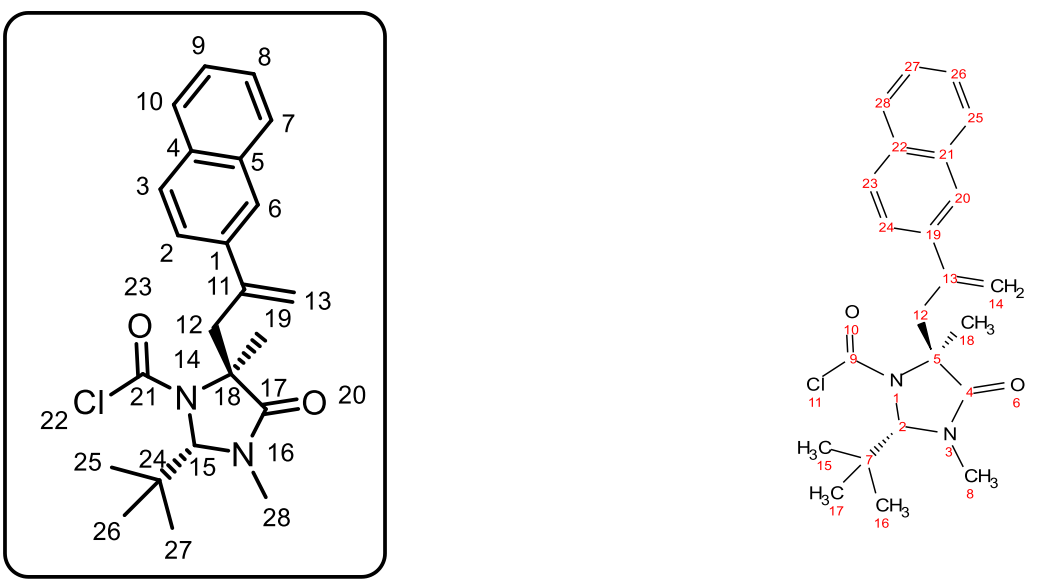

Following GP1, trans $N$-chloroformylimidazolidinone 1 a $(80.0 \mathrm{mg}, 0.0 .344 \mathrm{mmol}, 1.0$ eq.) was dissolved in dry THF $(3.44 \mathrm{~mL}, 0.1 \mathrm{M})$ and the solution was cooled to $-78{ }^{\circ} \mathrm{C}$ before the dropwise addition of KHMDS $\left(0.378 \mathrm{ml}, 1.0 \mathrm{M}\right.$ in THF, 1.1 eq.). The reaction was left to stir for $2 \mathrm{~min}$ at $-78{ }^{\circ} \mathrm{C}$. 2-(3-Bromoprop-1-en-2-yl)naphthalene $(93.5 \mathrm{mg}, 0.0 .378 \mathrm{mmol}, 1.1 \mathrm{eq}$.) was added and the reaction mixture stirred at $-78{ }^{\circ} \mathrm{C}$ for $2 \mathrm{~h}$. The reaction mixture was quenched with aqueous $\mathrm{HCl}(1.0 \mathrm{M})$ and extracted with EtOAc (x2). The combined organic layers were washed with brine, dried over $\mathrm{MgSO}_{4}$, filtered and the solvent was removed under reduced pressure. The crude material was purified by flash column chromatography $\left(\mathrm{SiO}_{2}\right.$, pet.ether/EtOAc, 7:3) to afford the title compound $(83.0 \mathrm{mg}$, $61 \%$ ) as a pale yellow oil. $\mathbf{R}_{f}=0.36$ (pet.ether/EtOAC $\left.=7: 3\right) ;[\alpha]_{\mathrm{D}}^{23}=-44\left(c=1\right.$ in $\left.\mathrm{CHCl}_{3}\right)$.

IR $V_{\max }=2971,2938(\mathrm{C}-\mathrm{H}), 1737,1712\left(\mathrm{C}=\mathrm{O} ;{ }^{1} \mathbf{H}\right.$ NMR $\left(400 \mathrm{MHz}, \mathrm{CDCl}_{3}\right)$ (mixture of rotamers A:B in a 0.45:0.55 ratio) $\delta 7.91-7.69(\mathrm{~m}, 4 \mathrm{H}, \mathrm{H}-6+\mathrm{H}-3+\mathrm{H}-7+\mathrm{H}-10$, rot. $\mathrm{A}+\operatorname{rot} . \mathrm{B}), 7.52-7.35(\mathrm{~m}, 3 \mathrm{H}, \mathrm{H}-2+$ $\mathrm{H}-8+\mathrm{H}-9$, rot. $\mathrm{A}+\operatorname{rot} . \mathrm{B}), 5.42(\mathrm{~d}, J=82.3 \mathrm{~Hz}, 2 \mathrm{H}, \mathrm{H}-13$, rot. $\mathrm{A}+\operatorname{rot} . \mathrm{B}), 4.68(\mathrm{~d}, J=15.7 \mathrm{~Hz}, 1 \mathrm{H}, \mathrm{H}-15$, rot. $A+$ rot. B), $3.58-3.28(\mathrm{~m}, 2 \mathrm{H}, \mathrm{H}-12$, rot. $A+\operatorname{rot} . B), 1.76(\mathrm{~d}, J=66.8 \mathrm{~Hz}, 3 \mathrm{H}, \mathrm{H}-28$, rot. $A+$ rot. $B)$, $1.69(\mathrm{~d}, J=31.7 \mathrm{~Hz}, 3 \mathrm{H}, \mathrm{H}-19$, rot. A + rot. B), 0.89 (d, J = 52.8 Hz, 9H H-25 + H-26 + H-27, rot. A + rot. B); ${ }^{13} \mathrm{C}$ NMR (126 MHz, CDCl 3 ) $\delta 171.33$ (C-17 rot. B), 171.12 (C-17, rot. A), 148.95 (C-21 rot. B), 
146.81 (C-21, rot. A), 143.90, 143.79, 137.81, 137.52, 133.33, 133.28, 133.04, 132.98, 128.20, $128.19,127.74,127.71,127.61,126.48,126.40,126.17,126.05,125.64,125.58,125.54,125.52$ (C-1 to $C-11$, rot. $A$ + rot. B), $122.82(C-13$, rot. B), 122.43 (C-13, rot. A), 82.92 (C-15, rot. A), 82.55 (C-15, rot. B), 68.08 (C-18, rot. A), 65.01 (C-18, rot. B), 44.50 (C-12, rot. B), 42.02 (C-12, rot. A), 39.59 (C-24, rot. B), 39.40 (C-24, rot. A), 30.82 (C-28, rot. B), 30.70 (C-28, rot. A), 27.65 (C-25 + C-26 + C-27, rot. A), 26.97 (C-25 + C-26 + C-27, rot. B), 24.40 (C-19, rot. B), 23.33 (C-19, rot. A); HRMS $\mathrm{C}_{23} \mathrm{H}_{27} \mathrm{CIN}_{2} \mathrm{O}_{2}$ [M $+\mathrm{H}]^{+}$calculated: 399.1834 found: 399.1841 .

(2R,5S)-2-(tert-butyl)-5-(2-(3-methoxyphenyl)allyl)-3,5-dimethyl-4-oxoimidazolidine-1-carbonyl chloride $2 \mathrm{~g}$
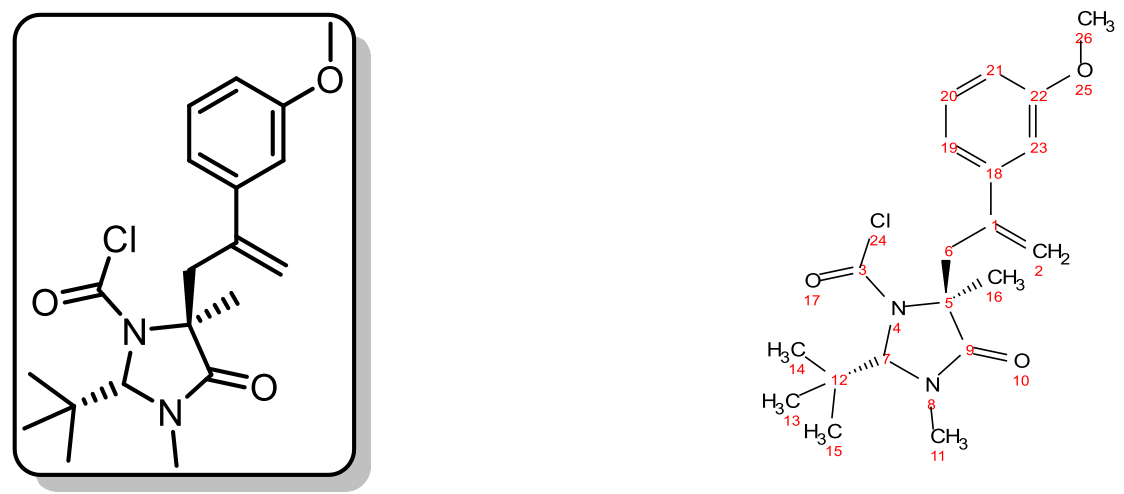

Following GP1, The title compound $\mathbf{2 g}$ was prepared from (2S,4R)-2-(tert-butyl)-1,4-dimethyl-5oxopyrrolidine-3-carbonyl chloride (150 mg, $0.64 \mathrm{mmol}, 1.0$ equiv.) and 1-(3-bromoprop-1-en-2-yl)3-methoxybenzene $(0.18 \mathrm{~mL}, 1.1 \mathrm{mmol}, 1.2$ equiv.). Colourless oil (170 mg, $73 \%)$. $\mathbf{R}_{\mathbf{f}} 0.25$ ( $\mathrm{SiO}_{2}$; pet. Ether/ EtOAc 4:1). $[\alpha]_{\mathrm{D}}^{\mathbf{2 6}}=-92^{\circ}\left(c=1\right.$ in $\left.\mathrm{CHCl}_{3}\right)$.

IR (neat, cm ${ }^{-1}$ ): $v_{\max } 2970(\mathrm{C}-\mathrm{H}), 1739(\mathrm{C}=\mathrm{O}), 1715(\mathrm{C}=\mathrm{O}), 1602 ;{ }^{1} \mathrm{H}$ NMR (500 MHz, $\left.\mathrm{CDCl}_{3}\right) \delta 7.25-$ $7.20(\mathrm{~m}, 1 \mathrm{H}, \mathrm{H}-\mathrm{Ar}$, rot. A+B), $6.90-6.82(\mathrm{~m}, 1 \mathrm{H}, \mathrm{H}-\mathrm{Ar}$, rot. A+B), 6.79 (tt, J = 2.7, $1.6 \mathrm{~Hz}, 1 \mathrm{H}, \mathrm{H}-\mathrm{Ar}$, rot $A+B), 6.77-6.76(m, 1 \mathrm{H}, \mathrm{H}-\mathrm{Ar}$, rot $\mathrm{A}+\mathrm{B}), 5.39-5.36(\mathrm{~m}, 0.92 \mathrm{H}, \mathrm{H}-2$, rot. B), $5.24-5.21(\mathrm{~m}, 1.08 \mathrm{H}, \mathrm{H}-$ 2, rot. B), $4.70(\mathrm{~s}, 0.46 \mathrm{H}, \mathrm{H}-7$, rot. B), $4.67(\mathrm{~s}, 0.54 \mathrm{H}, \mathrm{H}-7$, rot. A), $3.84(\mathrm{~s}, 3 \mathrm{H}, \mathrm{H}-26$, rot. A+B), $3.40-$ $3.39\left(\mathrm{~m}, 0.46 \mathrm{H}, \mathrm{H}-6_{B}\right.$, rot. $\left.B\right), 3.39-3.37\left(\mathrm{~m}, 0.54 \mathrm{H}, \mathrm{H}-6_{B}\right.$, rot. $\left.A\right), 3.36-3.34\left(\mathrm{~m}, 0.54 \mathrm{H}, \mathrm{H}-6_{A}\right.$, rot. $\left.A\right)$, $3.19-3.13\left(\mathrm{~m}, 0.46 \mathrm{H}, \mathrm{H}-6_{\mathrm{A}}\right.$, rot. B), $2.22(\mathrm{~s}, 1.38 \mathrm{H}, \mathrm{H}-11$, rot. B), 2.16 (s, 1.62H, H-11, rot. A), 1.69 (s, $1.62 \mathrm{H}, \mathrm{H}-16$, rot. A), 1.63 (s, 1.38H, H-16, rot. B), 0.98 (s, 4.14H, H-13, H-14, H-15, rot. B), 0.89 (s, 4.86H, H-13, H-14, H-15, rot. A). ${ }^{13} \mathrm{C}$ NMR (126 MHz, CDCl 3 ) $\delta 171.3$ (C-9, rot. A), 171.0 (C-9, rot. B), 159.3 (C-3, rot. B), 159.4 (C-3, rot. A), 148.6 (C-22, rot. A), 146.6 (C-22, rot. B), 143.6 (C-18, rot. B), 143.5 (C-18, rot. A), 141.7 (C-1, rot. B), 141.4 (C-1, rot. A), 129.2 (CH-Ar, rot. B), 129.1 (CH-Ar, rot. A), 122.1 (CH-Ar, rot. B), 121.8 (C-2, rot. A), 119.3 (C-2, rot. B), 119.3 (CH-Ar, rot. B), 114.1 (CH-Ar, rot. A), 113.7 (CH-Ar, rot. B), 112.1 (CH-Ar, rot. B), 111.7 (CH-Ar, rot. A), 82.8 (C-7, rot. B), 82.5 (C-7, rot. A), 67.8 (C-5, rot. B), 64.9 (C-5, rot. A), 55.3 (C-26, rot. A+B), 44.3 (C-6, rot. A), 41.8 (C-6, rot. B), 39.5 (C-12, rot. A), 39.3 (C-12, rot. B), 30.9 (C-11, rot. A), 30.7 (C-11, rot. B), 27.6 (C-13, C-14, C-15, rot. B), 26.9 (C-13, C-14, C-15, rot. A), 24.2 (C-16, rot. A), 23.1 (C-16, rot. B). HRMS $\mathrm{C}_{20} \mathrm{H}_{27} \mathrm{ClN}_{2} \mathrm{O}_{3}[\mathrm{M}+\mathrm{H}]^{+}$ calculated: 379.1783 , found 379.1774 . 
(2R,5S)-2-(tert-butyl)-5-(2-(4-fluorophenyl)allyl)-3,5-dimethyl-4-oxoimidazolidine-1-carbonyl chloride 2c
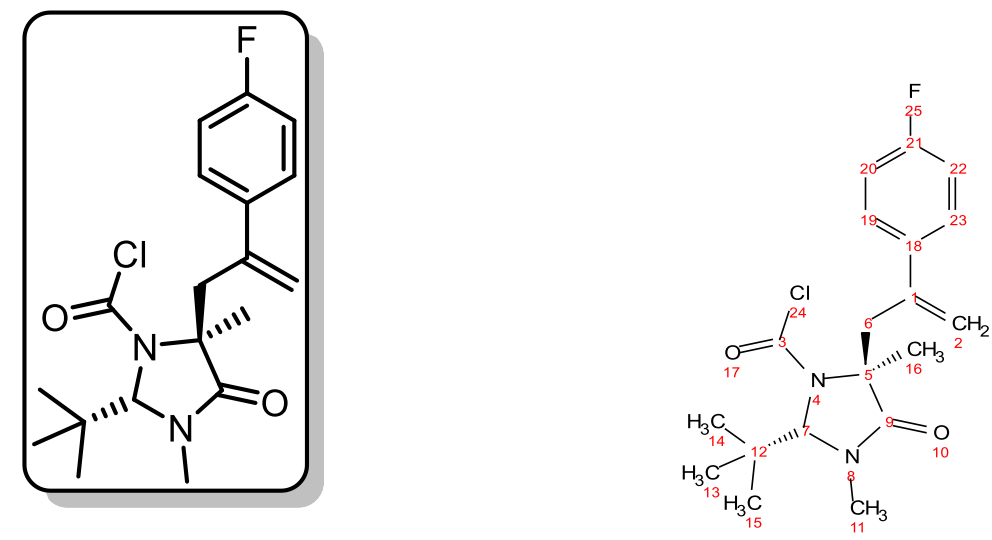

Following GP1, The title compound 2c was prepared from (2S,4R)-2-(tert-butyl)-1,4-dimethyl-5oxopyrrolidine-3-carbonyl chloride (200 mg, $1.0 \mathrm{mmol}, 1.0$ equiv.) and 1-(3-bromoprop-1-en-2-yl)-4fluorobenzene ( $0.22 \mathrm{~mL}, 1.2$ equiv.). Colourless oil ( $180 \mathrm{mg}, 58 \%) . \mathbf{R}_{\mathbf{f}} 0.34\left(\mathrm{SiO}_{2}\right.$; pet. Ether/ EtOAc $7 /$ 3). $[\alpha]_{\mathbf{D}}^{26}=-100^{\circ}\left(c=1\right.$ in $\left.\mathrm{CHCl}_{3}\right)$.

IR (neat, cm ${ }^{-1}$ ): $v_{\max } 2971(\mathrm{C}-\mathrm{H}), 1735$ (C=O), 1710 (C=O), 1605; ${ }^{1} \mathbf{H}$ NMR (500 MHz, CDCl $\left.)_{3}\right) \delta .28-$ $7.22(\mathrm{~m}, 2 \mathrm{H}, \mathrm{H}-19, \mathrm{H}-23$, rot. A+B), $7.01(\mathrm{t}, J=8.7 \mathrm{~Hz}, 2 \mathrm{H}, \mathrm{H}-20, \mathrm{H}-22$, rot. $\mathrm{A}+\mathrm{B}), 5.42-5.21(\mathrm{~m}, 1 \mathrm{H}, \mathrm{H}-$ $2_{A}$, rot. $\left.A+B\right), 5.19-5.13\left(m, 1 H, H-2_{B}\right.$ rot. $\left.A+B\right), 4.73(s, 0.45 \mathrm{H}, \mathrm{H}-7$, rot. $B), 4.69(\mathrm{~s}, 0.55 \mathrm{H}, \mathrm{H}-7$, rot. A), $3.37-3.35\left(\mathrm{~m}, 1 \mathrm{H}, \mathrm{H}-6_{A+B}\right.$, rot. A), $3.45-3.31\left(\mathrm{~m}, 0.55 \mathrm{H}, \mathrm{H}-6_{A+B}\right.$, rot. A), 3.13 (dd, J = 14.5, $1.3 \mathrm{~Hz}$, $0.45 \mathrm{H}, \mathrm{H}-6_{A+B}$, rot. B), 2.25 (s, 1.35H, H-11), $2.18(\mathrm{~s}, 1.65 \mathrm{H}, \mathrm{H}-11), 1.70(\mathrm{~s}, 1.65 \mathrm{H}, \mathrm{H}-16), 1.63(\mathrm{~s}, 1.35 \mathrm{H}$, $\mathrm{H}-16), 1.00$ (s, 4.05H, H-13, H-14, H-15, rot. B), 0.90 (s, 4.95H, H-13, H-14, H-15, rot. A). ${ }^{13} \mathrm{C}$ NMR (126 $\mathrm{MHz}, \mathrm{CDCl}_{3}$ ) $\delta 171.3$ (C-9, rot. A), 171.0 (C-9, rot. B), 163.5 (C-3, rot. A), 163.5 (C-3, rot. B), 161.6 (C-3, rot. A), 161.5 (C-3, rot. B), 148.9 (C-21, rot. A), 146.7 (C-21, rot. B), 142.8 (C-18, rot. B), 142.7 (C-18, rot. A), 136.6 (C-1, rot. A), 136.5 (C-1, rot. B), 136.3 (C-1, rot. A), 136.2 (C-1, rot. A), 128.9 (C-19/C-23, rot. B), 128.8 (C-19/C-23, rot. A+B), 128.7 (C-19/C-23, rot. A+B), 128.6 (C-19/C-23, rot. B), 122.5 (C-2, rot. A), 122.0 (C-2, rot. B), 115.2 (C-20/C-22, rot. A), 115.1 (C-20/C-22, rot. B), 115.0 (C-20/C-22, rot. A), 114.9 (C-20/C-22, rot. B), 82.9 (C-7, rot. B), 82.5 (C-7, rot. A), 68.0 (C-5, rot. B), 65.0 (C-5, rot. A), 44.3 (C-6, rot. A), 41.8 (C-6, rot. B), 39.6 (C-12, rot. A), 39.4 (C-12, rot. B), 31.0 (C-11, rot. A), 30.8 (C11, rot. B), 27.7 (C-13, C-14, C-15, rot. A), 27.0 (C-13, C-14, C-15, rot. A), 24.4 (C-16, rot. A), 23.3 (C16, rot. B). HRMS $\mathrm{C}_{19} \mathrm{H}_{24} \mathrm{ClFN}_{2} \mathrm{O}_{2}[\mathrm{M}+\mathrm{H}]^{+}$calculated: 367.1583 , found 367.1575 . 
(2R,5S)-5-(2-(4'-bromo-[1,1'-biphenyl]-4-yl)allyl)-2-(tert-butyl)-3,5-dimethyl-4-oxoimidazolidine-1carbonyl chloride $2 \mathrm{e}$
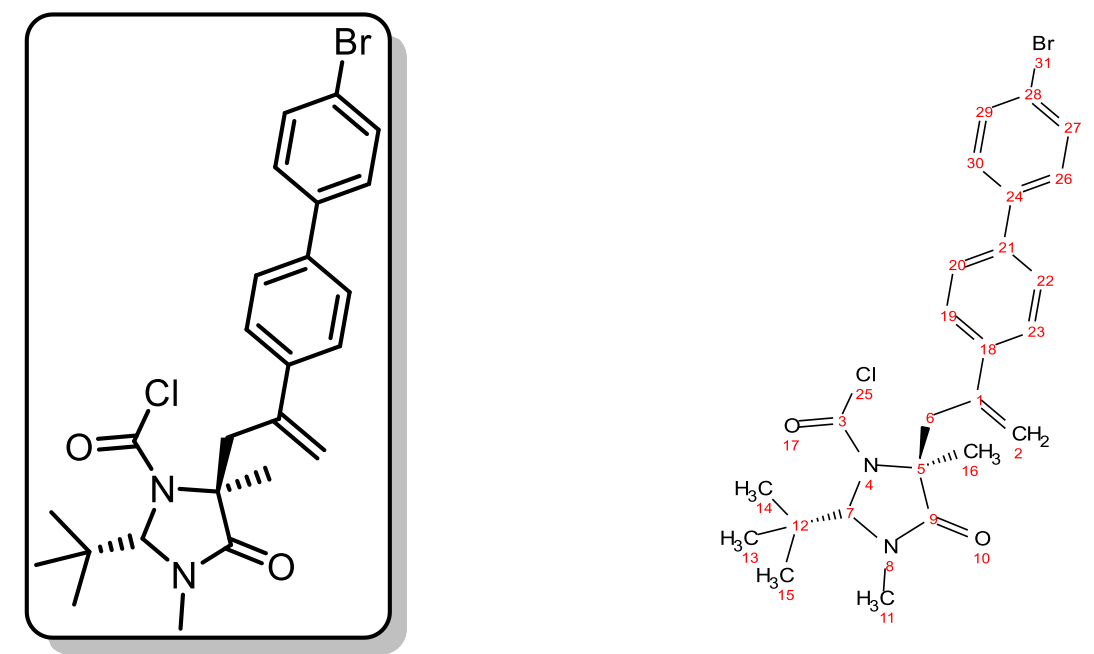

Following GP1, the title compound $\mathbf{2 e}$ was prepared from (2S,4R)-2-(tert-butyl)-1,4-dimethyl-5oxopyrrolidine-3-carbonyl chloride ( $200 \mathrm{mg}, 0.86 \mathrm{mmol}, 1.0$ equiv.) and 4-bromo-4'-(3-bromoprop-1en-2-yl)-1,1'-biphenyl (0.36 mL, $1.1 \mathrm{mmol}, 1.2$ equiv.). Colourless oil (320 mg, $0.79 \mathrm{mmol}, 80 \%) . \boldsymbol{R}_{\boldsymbol{f}}$ $0.47\left(\mathrm{SiO}_{2}\right.$; pet. Ether/ EtOAc 4:1). $[\alpha]_{\mathrm{D}}^{26}=-32^{\circ}\left(c=1\right.$ in $\left.\mathrm{CHCl}_{3}\right)$.

IR (neat, $\mathrm{cm}^{-1}$ ): $v_{\max } 2970(\mathrm{C}-\mathrm{H}), 1735(\mathrm{C}=0), 1713(\mathrm{C}=\mathrm{O}), 1602 ;{ }^{1} \mathrm{H}$ NMR (500 MHz, CDCl $)_{3}$ ) (mixture of rotamers $A: B$ in a 0.53:0.47 ratio) $\delta 7.58-7.51(\mathrm{~m}, 4 \mathrm{H}, \mathrm{H}-\mathrm{Ar}), 7.47-7.42(\mathrm{~m}, 2 \mathrm{H}, \mathrm{H}-\mathrm{Ar}), 7.38-7.33$ (m, $2 \mathrm{H}, \mathrm{H}-\mathrm{Ar}), 5.45-5.25(\mathrm{~m}, 2 \mathrm{H}, \mathrm{H}-2$, rot. $\mathrm{A}+$ rot. $\mathrm{B}), 4.74(\mathrm{~s}, 0.47 \mathrm{H}, \mathrm{H}-7$, rot. B), $4.72(\mathrm{~s}, 0.53 \mathrm{H}, \mathrm{H}-7$, rot. A), $3.45-3.43\left(\mathrm{~m}, 1 \mathrm{H}, \mathrm{H}-6_{A+B}\right.$, rot. $\left.A\right), 3.41\left(\mathrm{~d}, J=14.5 \mathrm{~Hz}, 0.55 \mathrm{H}, \mathrm{H}-6_{A+B}, \operatorname{rot} . A\right), 3.20(\mathrm{~d}, J=14.2$ $\mathrm{Hz}, 0.45 \mathrm{H}, \mathrm{H}-6_{\mathrm{A}+\mathrm{B}}$, rot. B), 2.19 (s, 1.41H, H-11, rot. B), 2.10 (s, 1.59H, H-11, rot. A), $1.72(\mathrm{~s}, 1.59 \mathrm{H}, \mathrm{H}-$ 16, rot. A), 1.65 (s, 1.41H, H-16, rot. B), 0.99 (s, 4.23H, H-13, H-14, H-15, rot. B), 0.89 (s, 4.77H, H-13, H-14, H-15, rot. A). ${ }^{13} \mathrm{C}$ NMR (126 MHz, CDCl 3 ) $\delta 171.3$ (C-9, rot. A), 171.1 (C-9, rot. B), 148.9 (C-3, rot. A), 146.8 (C-3, rot. B), 143.4 (C-Ar, rot. B), 143.2 (C-Ar, rot. A), 139.9 (C-Ar, rot. A), 139.6 (C-Ar, rot. B), 139.6 (C-Ar, rot. A), 139.5 (C-Ar, rot. B), 139.4 (C-Ar, rot. A), 139.2 (C-Ar, rot. B), 132.1 (CH-Ar, rot. A), 132.1 (CH-Ar, rot. B), 128.6 (CH-Ar, rot. B), 128.5 (CH-Ar, rot. A), 127.6 (CH-Ar, rot. A), 127.6 (CHAr, rot. B), 126.6 (CH-Ar, rot. B), 126.6 (CH-Ar, rot. A), 122.6 (C-Ar, rot. A), 122.1 (C-Ar, rot. B), 121.9 (C-Ar, rot. A), 121.8 (C-Ar, rot. B), 82.9 (C-7, rot. B), 82.5 (C-7, rot. A), 68.1 (C-5, rot. B), 65.0 (C-5, rot. A), 44.4 (C-6, rot. A), 41.8 (C-6, rot. B), 39.6 (C-12, rot. A), 39.4 (C-12, rot. B), 30.9 (C-11, rot. A), 30.7 (C-11, rot. B), 27.7 (C-13, C-14, C-15, rot. B), 27.0 (C-13, C-14, C-15, rot. A), 24.4 (C-16, rot. A), 23.3 (C-16, rot. B). HRMS $\mathrm{C}_{25} \mathrm{H}_{28} \mathrm{BrClN}_{2} \mathrm{O}_{2}[\mathrm{M}+\mathrm{H}]^{+}$calculated: 503.1095, found 503.1092. 
(2R,5S)-2-(tert-butyl)-5-(2-(3,4-dichlorophenyl)allyl)-3,5-dimethyl-4-oxoimidazolidine-1-carbonyl chloride 2d
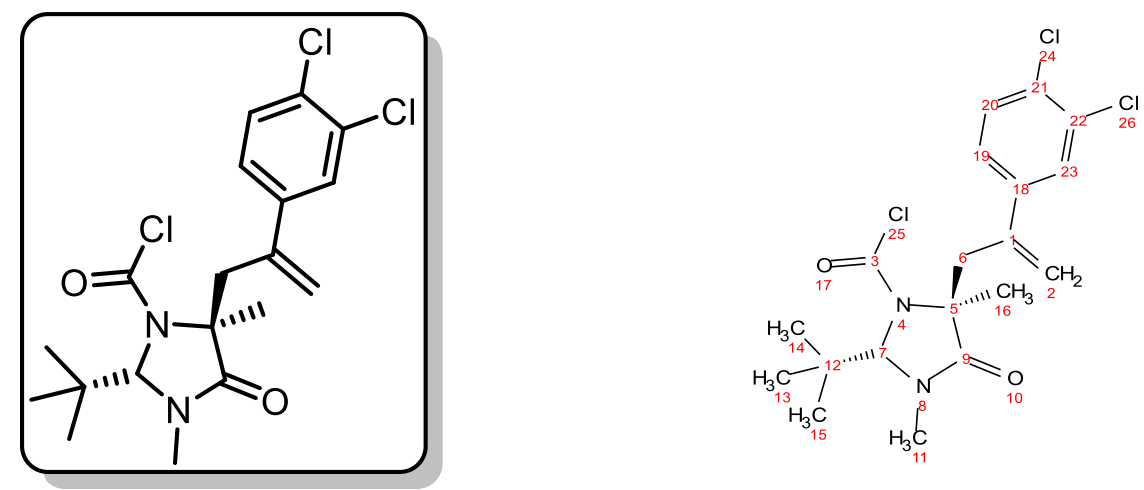

Following GP1, the title compound $\mathbf{2 d}$ was prepared from (2S,4R)-2-(tert-butyl)-1,4-dimethyl-5oxopyrrolidine-3-carbonyl chloride (150 mg, $0.64 \mathrm{mmol}, 1.0$ equiv.) and 4-(4-bromobut-1-en-2-yl)1,2-dichlorobenzene $\left(0.21 \mathrm{~mL}, 0.77 \mathrm{mmol}, 1.2\right.$ equiv.). Colourless oil (180 mg, 68\%). $\mathbf{R}_{\mathbf{f}} 0.30\left(\mathrm{SiO}_{2}\right.$; pet. Ether/ EtOAc 4:1).

$[\alpha]_{\mathrm{D}}^{26}=-60^{\circ}\left(c=1\right.$ in $\left.\mathrm{CHCl}_{3}\right)$. IR (neat, $\left.\mathrm{cm}^{-1}\right): v_{\max } 2988(\mathrm{C}-\mathrm{H}), 1730(\mathrm{C}=\mathrm{O}), 1710(\mathrm{C}=\mathrm{O}), 1601$.

${ }^{1} \mathrm{H}$ NMR $\left(500 \mathrm{MHz}, \mathrm{CDCl}_{3}\right) \delta 7.39$ (d, $J=8.3 \mathrm{~Hz}, 1 \mathrm{H}, \mathrm{H}-20$, rot. A+B), 7.35 (dd, $J=3.3,2.1 \mathrm{~Hz}, 1 \mathrm{H}, \mathrm{H}-23$, rot. A+B), 7.10 (ddd, $J=10.4,8.3,2.1 \mathrm{~Hz}, 1 \mathrm{H}, \mathrm{H}-19$, rot. A+B), 5.43 (d, J = $1.1 \mathrm{~Hz}, 0.49 \mathrm{H}, \mathrm{H}-2_{B}$, rot. B), $5.28\left(\mathrm{~d}, J=1.5 \mathrm{~Hz}, 0.51 \mathrm{H}, \mathrm{H}-2_{\mathrm{A}}\right.$, rot. A), $5.24\left(\mathrm{~d}, J=1.2 \mathrm{~Hz}, 1 \mathrm{H}, \mathrm{H}-2_{\mathrm{A}+\mathrm{B}}\right.$, rot. $\left.\mathrm{A}+\mathrm{B}\right), 4.75(\mathrm{~s}, 0.51 \mathrm{H}, \mathrm{H}-7$, rot. A), $4.72(\mathrm{~s}, 0.49 \mathrm{H}, \mathrm{H}-7$, rot. $B), 3.41\left(\mathrm{dd}, J=22.4,14.8 \mathrm{~Hz}, 1 \mathrm{H}, \mathrm{H}-6_{A+B}, \operatorname{rot} . \mathrm{A}+\mathrm{B}\right), 3.33-3.27(\mathrm{~m}$, $0.49 \mathrm{H}, \mathrm{H}-6_{B}$, rot. B), 3.07 (dd, J = 14.6, $1.3 \mathrm{~Hz}, 0.51 \mathrm{H}, \mathrm{H}-6_{\mathrm{A}}$, rot. A), $2.33(\mathrm{~s}, 1.53 \mathrm{H}, \mathrm{H}-11$, rot. A), 2.25 (s, 1.47H, H-11, rot. B), 1.70 (s, 1.47H, H-16, rot. B), 1.63 (s, 1.53H, H-16, rot. A), 1.01 (s, 4.59H, H-13, $\mathrm{H}-14, \mathrm{H}-15$, rot. A), 0.91 (s, 4.41H, H-13, H-14, H-15, rot. B). ${ }^{13} \mathrm{C}$ NMR (126 MHz, CDCl 3 ) $\delta 171.1$ (C-9, rot. B), 170.9 (C-9, rot. A), 148.7 (C-3, rot. A), 146.6 (C-3, rot. B), 141.9 (C-1, rot. A), 141.7 (C-1, rot. B), 140.6 (C-18, rot. A), 140.2 (C-18, rot. B), 132.3 (C-Ar, rot. A/B), 132.2 (C-Ar, rot. A/B), 131.9 (C-Ar, rot. $A / B$ ), 131.7 (C-Ar, rot. $A / B$ ), 130.0 (C-20, rot. B), 130.1 (C-20, rot. A), 128.8 (C-23, rot. A), 128.9 (C-23, rot. $B$ ), 126.5 (C-19, rot. $A+B), 123.4$ (C-2 $2_{A+B}$, rot. $\left.A / B\right), 122.9$ (C-2 $2_{A+B}$, rot. $\left.A / B\right), 83.0$ (C-7, rot. A), 82.6 (C-7, rot. B), 68.0 (C-5, rot. A), 65.0 (C-5, rot. B), 44.0 (C-6, rot. B), 41.4 (C-6, rot. A), 39.7 (C12, rot. B), 39.4 (C-12, rot. A), 31.0 (C-11, rot. B), 30.9 (C-11, rot. A), 27.7 (C-13, C-14, C-15, rot. A), 27.0 (C-13, C-14, C-15, rot. B), 24.3 (C-16, rot. B), 23.2 (C-16, rot. A). HRMS $\mathrm{C}_{19} \mathrm{H}_{23} \mathrm{Cl}_{3} \mathrm{~N}_{2} \mathrm{O}_{2}[\mathrm{M}+\mathrm{H}]^{+}$ calculated: 417.0898 , found 417.0897 . 


\section{$\underline{\text { Lactams }}$}

(3S,8aS)-3-(tert-butyl)-2,8a-dimethyl-2,3,8,8a-tetrahydroimidazo[1,5-a]pyridine-1,5-dione 3a:
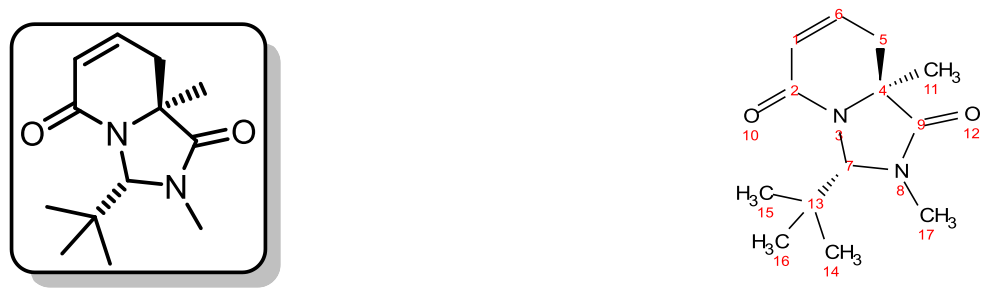

and (3S,8aS)-3-(Tert-butyl)-2,8a-dimethyl-2,3,6,8a-tetrahydroimidazo[1,5-a]pyridine-1,5-dione 4a:
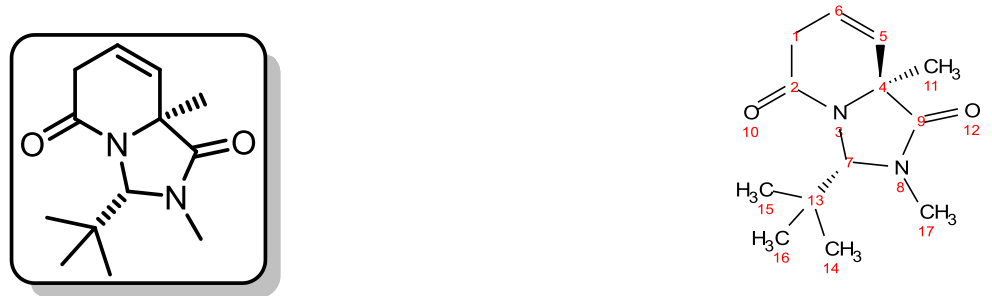

Following GP2, $N$-chloroformylimidazolidinone 1a (1.00 g, $3.67 \mathrm{mmol}, 1.0$ eq.) was dissolved in dry MeCN (15.0 mL, $0.2 \mathrm{M}$ ) before the addition of potassium iodide $(670 \mathrm{mg}, 4.03 \mathrm{mmol}, 1.1 \mathrm{eq}$.$) and$ 2,6-lutidine ( $468 \mu \mathrm{L}, 4.03 \mathrm{mmol}, 1.1$ eq.). The reaction was heated to $150{ }^{\circ} \mathrm{C}$ in a microwave reactor for $2 \mathrm{~h}$. The reaction was quenched with aqueous $\mathrm{HCl}(1.0 \mathrm{M})$. The reaction mixture was partitioned between $\mathrm{H}_{2} \mathrm{O}$ and EtOAc. The aqueous phase was further extracted with EtOAc (x2) and the combined organic phases were washed with brine, dried over $\mathrm{MgSO}_{4}$, filtered and the solvent was removed under reduced pressure. The crude product (43:57, 3a:4a isomeric ratio by $\left.{ }^{1} \mathrm{H} N M R\right)$ was purified by flash column chromatography $\left(\mathrm{SiO}_{2}\right.$, pet.ether/EtOAc, 4:6) to yield;

\section{Conjugated isomer $3 a$}

The title compound 3a was prepared from (2S,4R)-4-allyl-2-(tert-butyl)-1,4-dimethyl-5oxopyrrolidine-3-carbonyl chloride (41 $\mathrm{mg}, 0.15 \mathrm{mmol}, 1.0$ equiv.) according to the general procedure. The reaction was finished after $2 \mathrm{~h}$. White solid $(13 \mathrm{mg}, 0.06 \mathrm{mmol}, 39 \%)$. m.p. $=103-$ $106^{\circ} \mathrm{C} . \mathbf{R}_{\mathbf{f}} 0.40\left(\mathrm{SiO}_{2}\right.$; pet. Ether/ EtOAc 4/6). $[\boldsymbol{\alpha}]_{\mathrm{D}}^{23}=-156^{\circ}\left(c=1\right.$ in $\left.\mathrm{CHCl}_{3}\right)$.). IR (neat, $\left.\mathrm{cm}^{-1}\right): v_{\max } 2978$

(C-H), 1704 (C=O), 1672 (C=O), 1389. ${ }^{1} \mathrm{H}$ NMR (400 MHz, Chloroform-d) $\delta 6.44$ (ddd, $J=9.8,6.4,2.3$ $\mathrm{Hz}, 1 \mathrm{H}, \mathrm{H}-6), 5.95$ (ddd, J = 9.8, 3.1, $0.6 \mathrm{~Hz}, 1 \mathrm{H}, \mathrm{H}-1$ ), 5.15 (s, 1H, H-7), 2.96 (s, 3H, H-17), 2.45 (ddd, J $=17.4,6.4,0.6 \mathrm{~Hz}, 1 \mathrm{H}, \mathrm{H}-5), 2.31$ (dddd, $J=17.4,3.1,2.3,0.8 \mathrm{~Hz}, 1 \mathrm{H}, \mathrm{H}-5), 1.51(\mathrm{~s}, 3 \mathrm{H}, \mathrm{H}-11), 1.02$ (s, 9H, H-14 + H-15 + H-16). ${ }^{13} \mathrm{C}$ NMR (101 MHz, CDCl 3 ) $\delta 173.7$ (C-9), 163.0 (C-2), 136.3 (C-6), 125.2 (C1), 80.9 (C-7), 60.5 (C-4), 37.8 (C-13), 34.2 (C-5), 31.2 (C-17), 27.5 (C-14 + C-15 + C-16), 22.8 (C-11). HRMS $\left(\mathrm{EI}^{+}\right): \mathrm{m} / z$ calcd. for $\mathrm{C}_{13} \mathrm{H}_{20} \mathrm{~N}_{2} \mathrm{O}_{2} \mathrm{Na}[\mathrm{M}+\mathrm{Na}]^{+} 259.1416$, found 259.1409 .

Data in agreement with reported values. ${ }^{2}$

and; 


\section{Unconjugated 4a}

The title compound $4 a$ was prepared from (2S,4R)-2-(tert-butyl)-1,4-dimethyl-4-(2-methylallyl)-5oxopyrrolidine-3-carbonyl chloride (41 $\mathrm{mg}, 0.15 \mathrm{mmol}, 1.0$ equiv.) according to the general procedure. The reaction was finished after $2 \mathrm{~h}$. (17 mg, $0.07 \mathrm{mmol}, 50 \%)$. m.p. $=107-109^{\circ} \mathrm{C} \cdot \boldsymbol{R}_{f} 0.70$ $\left(\mathrm{SiO}_{2}\right.$; pet. Ether/ EtOAc 4/6). $[\alpha]_{\mathbf{D}}^{24}=-28^{\circ}\left(c=1\right.$ in $\left.\mathrm{CHCl}_{3}\right)$. IR (neat, $\left.\mathbf{c m}^{-1}\right): v_{\max } 2978(\mathrm{C}-\mathrm{H}), 1706$

(C=O), 1677 (C=O). ${ }^{1} \mathrm{H}$ NMR (400 MHz, CDCl $) \delta 6.11$ (dd, $\left.J=9.2,3.5 \mathrm{~Hz}, 1 \mathrm{H}, \mathrm{H}-5\right), 5.82$ (ddd, J = 9.2, 6.0, $2.1 \mathrm{~Hz}, 1 \mathrm{H}, \mathrm{H}-6), 5.13(\mathrm{~s}, 1 \mathrm{H}, \mathrm{H}-7), 3.03-2.79(\mathrm{~m}, 5 \mathrm{H}, \mathrm{H}-1+\mathrm{H}-17), 1.48(\mathrm{~s}, 3 \mathrm{H}, \mathrm{H}-11), 1.02(\mathrm{~s}, 9 \mathrm{H}$, $\mathrm{H}-14+\mathrm{H}-15+\mathrm{H}-16) .{ }^{13} \mathrm{C}$ NMR (101 MHz, CDCl $\left.{ }_{3}\right) \delta 171.4$ (C-9), 170.2 (C-2), 131.1 (C-5), 121.4 (C-6), 80.8 (C-7), 64.5 (C-4), 37.2 (C-13), 33.3 (C-1), 31.3 (C-17), 27.5 (C-14 + C-15 + C-16), 24.8 (C-11). HRMS $\left(\mathrm{EI}^{+}\right): \mathrm{m} / z$ calcd. for $\mathrm{C}_{13} \mathrm{H}_{20} \mathrm{~N}_{2} \mathrm{O}_{2} \mathrm{Na}[\mathrm{M}+\mathrm{Na}]^{+} 259.1416$, found 259.1414 .

\section{Isomerisation of 4a to 3a}

Following GP4, 4a (250.0 mg, $1.06 \mathrm{mmol}, 1.0$ eq.) was dissolved in dry MeCN (5.0 mL, $0.2 \mathrm{M}$ ) before the addition of $\mathrm{DBU}\left(475 \mu \mathrm{L}, 3.17 \mathrm{mmol}, 3.0\right.$ eq.). The reaction was heated to $150^{\circ} \mathrm{C}$ in a microwave reactor for $2 \mathrm{~h}$. The reaction was quenched with aqueous $\mathrm{HCl}(1.0 \mathrm{M})$. The reaction mixture was partitioned between $\mathrm{H}_{2} \mathrm{O}$ and EtOAc. The aqueous phase was further extracted with EtOAc $(x 2)$ and the combined organic phases were washed with brine, dried over $\mathrm{MgSO}_{4}$, filtered and the solvent was removed under reduced pressure. The crude product was purified by flash column chromatography $\left(\mathrm{SiO}_{2}\right.$, pet.ether/EtOAc, 4:6) to yield 3a $(162.6 \mathrm{mg}, 65 \%)$ as a white solid. Data in agreement with those which are reported above.

(3S,7aS)-6-((E)-benzylidene)-3-(tert-butyl)-2,7a-dimethyltetrahydro-1H-pyrrolo[1,2-c]imidazole1,5(6H)-dione: $E-7 \mathrm{i}$
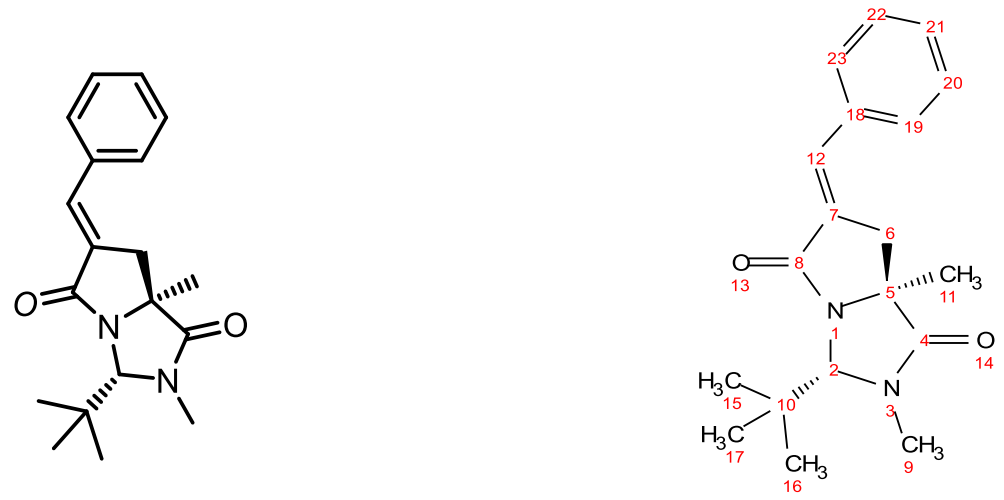

Following GP2, the title compound $\boldsymbol{E}$-7i was prepared from (2S,5S)-2-(tert-butyl)-5-cinnamyl-3,5dimethyl-4-oxoimidazolidine-1-carbonyl chloride $2 \mathbf{i}(52 \mathrm{mg}, 0.15 \mathrm{mmol}, 1.0$ equiv.). The reaction was finished after $2 \mathrm{~h}$. White solid (30 mg, $0.1 \mathrm{mmol}, 65 \%)$. m.p. $=99-103{ }^{\circ} \mathrm{C} . \boldsymbol{R}_{f} 0.50\left(\mathrm{SiO}_{2} ;\right.$ pet. Ether/ EtOAc 7/3). [ $\alpha]_{\mathbf{D}}^{23}=+116^{\circ}\left(c=1\right.$ in $\mathrm{CHCl}_{3}$ ). IR (neat, $\left.\mathrm{cm}^{-1}\right)$ : $v_{\max } 2997(\mathrm{C}-\mathrm{H}), 1699(\mathrm{C}=\mathrm{O}), 1649(\mathrm{C}=\mathrm{O})$,

1334. ${ }^{1} \mathrm{H}$ NMR $\left(400 \mathrm{MHz}, \mathrm{CDCl}_{3}\right) \delta 7.60-7.11(\mathrm{~m}, 6 \mathrm{H}, \mathrm{H}-\mathrm{Ar}+\mathrm{H}-12), 4.77$ (s, 1H, H-2), 3.31 (dd, $J=$ 17.6, 3.5 Hz, 1H, H-6), 3.03 (dd, $J=17.6,2.2 \mathrm{~Hz}, 1 \mathrm{H}, \mathrm{H}-6), 2.93$ (s, 3H, H-9), 1.51 (s, 3H, H-11), 1.06 (s, 9H, H-15 + H-16 + H-17). ${ }^{13} \mathrm{C}$ NMR (101 MHz, Chloroform-d) $\delta 176.2$ (C-8), 174.8 (C-4), 135.0 (C-18), 133.5 (C-12), 129.9 (C-7), 129.8 (C-19 + C-23), 129.2 (C-21), 128.8 (C-20 + C-22), 84.4 (C-2), 63.5 (C-5), 40.2 (C-6), 36.4 (C-10), 31.2 (C-9), 27.3 (C-11), 27.2 (C-15 + C-16 + C-17). HRMS (El+): $\mathrm{m} / \mathrm{z}$ calcd. for $\mathrm{C}_{19} \mathrm{H}_{24} \mathrm{~N}_{2} \mathrm{O}_{2} \mathrm{Na}[\mathrm{M}+\mathrm{Na}]^{+} 335.1729$, found 335.1740 . 
(3S,7aS)-6-((Z)-benzylidene)-3-(tert-butyl)-2,7a-dimethyltetrahydro-1H-pyrrolo[1,2-c]imidazole1,5(6H)-dione: Z-7i
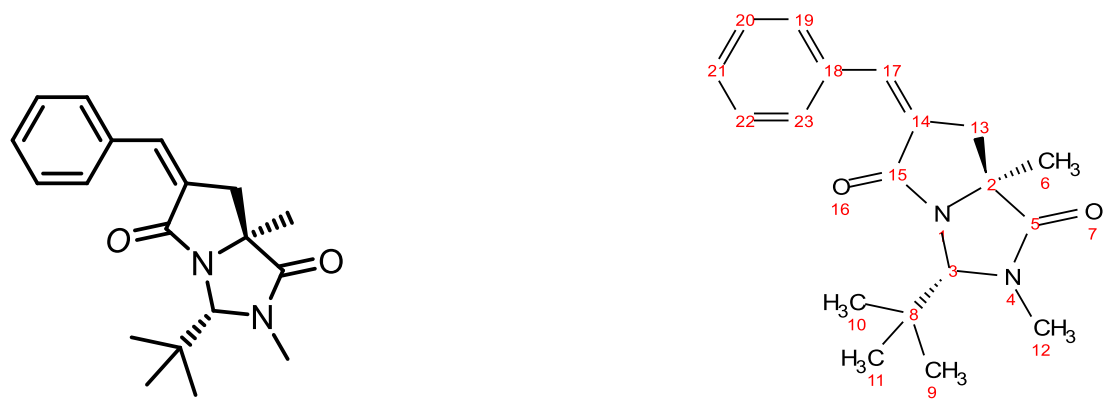

Following GP2, the title compound $\mathbf{Z}-7 \mathbf{i}$ was prepared from $\mathbf{2 i}(52 \mathrm{mg}, 0.15 \mathrm{mmol}, 1.0$ equiv.). The reaction was finished after $2 \mathrm{~h}$. White solid (13 mg, $0.04 \mathrm{mmol}, 28 \%$ ). m.p. $=105-110{ }^{\circ} \mathrm{C} . \boldsymbol{R}_{f} 0.40$ $\left(\mathrm{SiO}_{2}\right.$; pet. Ether/ EtOAc $\left.7 / 3\right)$. $[\alpha]_{\mathrm{D}}^{\mathbf{2 4}}=-12^{\circ}\left(c=1\right.$ in $\left.\mathrm{CHCl}_{3}\right)$.

IR (neat, $\left.\mathrm{cm}^{-1}\right): \mathrm{v}_{\max } 2973(\mathrm{C}-\mathrm{H}), 1697(\mathrm{C}=\mathrm{O}), 1648(\mathrm{C}=\mathrm{O}), 1256 .{ }^{1} \mathrm{H}$ NMR $\left(400 \mathrm{MHz}, \mathrm{CDCl}_{3}\right) \delta 7.88-$ $7.63(\mathrm{~m}, 2 \mathrm{H}, \mathrm{H}-19+\mathrm{H}-23), 7.46-7.13(\mathrm{~m}, 3 \mathrm{H}, \mathrm{H}-20+\mathrm{H}-21+\mathrm{H}-22), 6.68(\mathrm{dd}, J=3.2,1.2 \mathrm{~Hz}, 1 \mathrm{H}, \mathrm{H}-$ 17), $4.80(\mathrm{~s}, 1 \mathrm{H}, \mathrm{H}-3$ ), 3.15 (dd, $J=15.7,3.1 \mathrm{~Hz}, 1 \mathrm{H}, \mathrm{H}-13$ ), 2.93 (s, 3H, H-12), 2.79 (dd, $J=15.7,1.4$ $\mathrm{Hz}, 1 \mathrm{H}, \mathrm{H}-13), 1.54$ (s, 3H, H-6), 1.02 (s, 9H, H-9 + H-10 + H-11). ${ }^{13} \mathrm{C}$ NMR (101 MHz, Chloroform-d) $\delta$ 174.6 (C-5), 172.6 (C-15), 135.6 (C-17), 134.2 (C-18), 130.2 (C-19 + C-23), 130.0 (C-14), 128.9 (C-21), 128.2 (C-20 + C-22), 83.2 (C-3), 62.4 (C-2), 44.9 (C-13), 36.5 (C-8), 31.2 (C-12), 27.0 (C-9 + C-10 + C11), 25.5 (C-6). HRMS $\left(\mathrm{EI}^{+}\right): \mathrm{m} / \mathrm{z}$ calcd. for $\mathrm{C}_{19} \mathrm{H}_{24} \mathrm{~N}_{2} \mathrm{O}_{2} \mathrm{Na}[\mathrm{M}+\mathrm{Na}]^{+} 335.1729$, found 335.1718 .

(3S,7aS)-3-(tert-butyl)-6-ethylidene-2,7a-dimethyltetrahydro-1H-pyrrolo[1,2-c]imidazole-1,5(6H)dione: $7 \mathrm{j}$<smiles>CC=C1C[C@]2(C)C(=O)N(C)[C@@H](C(C)(C)C)N2C1=O</smiles><smiles>CN=[IH]1N[Te]2(C)(=O)N(C)[C@H](C3(C)CC3)N2[Y]1=O</smiles>

The title compound $7 \mathbf{j}$ was prepared from (2S,5S)-5-((E)-but-2-en-1-yl)-2-(tert-butyl)-3,5-dimethyl-4oxoimidazolidine-1-carbonyl chloride (43 $\mathrm{mg}, 0.15 \mathrm{mmol}, 1.0$ equiv.) according to the general procedure. The reaction was finished after $2 \mathrm{~h}$. White solid (14 mg, $0.05 \mathrm{mmol}, 36 \%)$. m.p.= $105-$ $113^{\circ} \mathrm{C} . \boldsymbol{R}_{f} 0.43\left(\mathrm{SiO}_{2}\right.$; pet. Ether/ EtOAc 5/5). $[\alpha]_{\mathrm{D}}^{24}=-28^{\circ}\left(c=1\right.$ in $\left.\mathrm{CHCl}_{3}\right)$.

IR (neat, cm $\left.{ }^{-1}\right)$ : $v_{\max } 2972(\mathrm{C}-\mathrm{H}), 1696(\mathrm{C}=\mathrm{O}), 1642(\mathrm{C}=\mathrm{O}), 1246 .{ }^{1} \mathrm{H}$ NMR (400 MHz, Chloroform-d) $\delta$ 6.52 (qdd, $J=7.1,3.5,2.2 \mathrm{~Hz}, 1 \mathrm{H}, \mathrm{H}-17$ ), $4.70(\mathrm{~s}, 1 \mathrm{H}, \mathrm{H}-3), 2.90$ (s, 3H, H-6), $2.89-2.82(\mathrm{~m}, 1 \mathrm{H}, \mathrm{H}-13$ ), 2.67 (ddq, $J=17.0,2.7,1.4 \mathrm{~Hz}, 1 \mathrm{H}, \mathrm{H}-13$ ), 1.72 (ddd, $J=7.2,2.5,1.3 \mathrm{~Hz}, 3 \mathrm{H}, \mathrm{H}-18$ ), 1.48 (s, 3H, H-8), 1.02 (s, 9H, H-10+ H-11 + H-12). ${ }^{13} \mathrm{C}$ NMR (101 MHz, Chloroform-d) $\delta 175.0$ (C-5), 174.9 (C-15), 131.8 (C-14), 131.7 (C-17), 84.0 (C-3), 63.0 (C-2), 37.6 (C-13), 36.3 (C-9), 31.1 (C-6), 27.1 (C-10 + C-11 + C12), 27.0 (C-8), 15.1 (C-18). HRMS (El+): $m / z$ calcd. for $\mathrm{C}_{14} \mathrm{H}_{23} \mathrm{~N}_{2} \mathrm{O}_{2}[\mathrm{M}+\mathrm{H}]^{+}$251.1754, found 251.1751. 
(3S,7aS)-3-(tert-butyl)-2,7a-dimethyl-6-vinyltetrahydro-1H-pyrrolo[1,2-c]imidazole-1,5(6H)-dione 8j:

\section{And,}

(3S,8aS)-3-(tert-butyl)-2,6,8a-trimethyl-2,3,8,8a-tetrahydroimidazo[1,5-a]pyridine-1,5-dione 3j:<smiles>C=C[C@H]1C[C@]2(C)C(=O)N(C)[C@H](C(C)(C)C)N2C1=O</smiles><smiles>C=CN1N[C@@]2(C)C(=O)N(C)[C@H](C(C)(C)C)N2N1C</smiles><smiles>CC1=CC[C@@]2(C)C(=O)N(C)[C@@H](C(C)(C)C)N2C1=O</smiles><smiles>CC1=NN[C@@]2(C)C(=O)N(C)N(C)[C@@]2(C(C)(C)C)N1C</smiles>

Following GP2, the title compounds $\mathbf{8} \mathbf{j}$ and $\mathbf{3} \mathbf{j}$ were prepared from (2S,5S)-5-((E)-but-2-en-1-yl)-2(tert-butyl)-3,5-dimethyl-4-oxoimidazolidine-1-carbonyl chloride (43 $\mathrm{mg}, 0.15 \mathrm{mmol}, 1.0$ equiv.) according to the general procedure. The reaction was finished after $2 \mathrm{~h}$. Isolated as a mixture. Colourless oil (19 mg, $0.08 \mathrm{mmol}, 47 \%) . \boldsymbol{R}_{\boldsymbol{f}} 0.54\left(\mathrm{SiO}_{2}\right.$; pet. Ether/ EtOAc 5/5). ${ }^{1} \mathrm{H}$ NMR spectra for compound 8j (400 MHz, Chloroform-d) $\delta 5.83$ (ddd, $J=17.1,10.5,6.4 \mathrm{~Hz}, 1 \mathrm{H}, \mathrm{H}-17$ ), 5.17 (dt, $J=$ $10.5,1.3 \mathrm{~Hz}, 1 \mathrm{H}, \mathrm{H}-18$ ), 5.08 (dt, $J=17.3,1.4 \mathrm{~Hz}, 1 \mathrm{H}, \mathrm{H}-18$ ), 4.71 (s, $1 \mathrm{H}, \mathrm{H}-3$ ), 3.48 (dddt, $J=13.4,7.7$, 6.3, $1.6 \mathrm{~Hz}, 1 \mathrm{H}, \mathrm{H}-14), 2.90(\mathrm{~s}, 3 \mathrm{H}, \mathrm{H}-6), 2.33(\mathrm{dd}, J=12.4,7.4 \mathrm{~Hz}, 1 \mathrm{H}, \mathrm{H}-13), 2.08-1.88(\mathrm{~m}, 1 \mathrm{H}, \mathrm{H}-$ 13), $1.56-1.51$ (s, 3H, H-8), 0.97 (s, 9H, H-10 + H-11 + H-12). ${ }^{13} \mathrm{C}$ NMR (101 MHz, Chloroform-d) $\delta$ 180.5 (C-5), 174.6 (C-15), 133.2 (C-17), 118.1 (C-18), 82.3 (C-3), 63.2 (C-2), 45.5 (C-14), 41.4 (C-13), 36.5 (C-9), 31.1 (C-6), 26.7 (C-10 + C-11 + C-12), 23.8 (C-8). ${ }^{1}$ H NMR spectra for compound 3j (400 $\mathrm{MHz}$, Chloroform-d) $\delta 5.96$ (qdd, $J=7.3,3.0,1.4 \mathrm{~Hz}, 1 \mathrm{H}, \mathrm{H}-14), 4.72(\mathrm{~s}, 1 \mathrm{H}, \mathrm{H}-3), 2.90(\mathrm{~m}, 4 \mathrm{H}, \mathrm{H}-6+\mathrm{H}-$ 13), $2.65-2.55(\mathrm{~m}, 1 \mathrm{H}, \mathrm{H}-13), 2.04(\mathrm{~m}, 3 \mathrm{H}, \mathrm{H}-18), 1.46(\mathrm{~s}, 3 \mathrm{H}, \mathrm{H}-8), 1.01(\mathrm{~s}, 9 \mathrm{H}, \mathrm{H}-10+\mathrm{H}-11+\mathrm{H}-12)$. ${ }^{13} \mathrm{C}$ NMR (101 MHz, Chloroform-d) $\delta 174.9$ (C-5), 174.3 (C-16), 134.2 (C-14), 129.9 (C-15), 83.3 (C-3), 62.5 (C-2), 42.2 (C-13), 36.4 (C-9), 31.2 (C-6), 27.0 (C-10 + C-11 + C-12), 25.8 (C-8), 13.9 (C-18). HRMS $\left(\mathrm{EI}^{+}\right): \mathrm{m} / z$ calcd. for $\mathrm{C}_{14} \mathrm{H}_{23} \mathrm{~N}_{2} \mathrm{O}_{2}[\mathrm{M}+\mathrm{H}]^{+} 251.1754$, found 251.1754 . 
<smiles>CC1=CC(=O)N2[C@@H](C(C)(C)C)N(C)C(=O)[C@]2(C)C1</smiles><smiles>CC1=CC(=O)N2C(N([13CH3])C(=O)C2(C)C)N1C(N)=O</smiles>

Following GP2, the title compound $3 \mathrm{~h}$ was prepared from (2S,4R)-4-allyl-2-(tert-butyl)-1,4-dimethyl5-oxopyrrolidine-3-carbonyl chloride $(43 \mathrm{mg}, 0.15 \mathrm{mmol}, 1.0$ equiv.). The reaction was finished after 2 h. White solid (9 mg, $0.04 \mathrm{mmol}, 24 \%)$. m.p. $=134-139{ }^{\circ} \mathrm{C} . \boldsymbol{R}_{f} 0.23\left(\mathrm{SiO}_{2}\right.$; pet. Ether/ EtOAc 4/6). $[\alpha]_{\mathrm{D}}^{24}=-168^{\circ}\left(c=1\right.$ in $\left.\mathrm{CHCl}_{3}\right)$.

IR (neat, cm $\left.{ }^{-1}\right): v_{\max } 2974(\mathrm{C}-\mathrm{H}), 1705(\mathrm{C}=\mathrm{O}), 1672(\mathrm{C}=\mathrm{O}), 1380 .{ }^{1} \mathrm{H}$ NMR (400 MHz, Chloroform-d) $\delta$ 5.78 (dd, $J=2.7,1.4 \mathrm{~Hz}, 1 \mathrm{H}, \mathrm{H}-1), 5.17(\mathrm{~s}, 1 \mathrm{H}, \mathrm{H}-7), 3.01$ (s, 3H, H-18), 2.41 (d, J=16.0 Hz, 1H, H-5), $2.29(\mathrm{~d}, J=16.9 \mathrm{~Hz}, 1 \mathrm{H}, \mathrm{H}-5), 1.93(\mathrm{t}, J=1.4 \mathrm{~Hz}, 3 \mathrm{H}, \mathrm{H}-12), 1.54(\mathrm{~s}, 3 \mathrm{H}, \mathrm{H}-14), 1.07(\mathrm{~s}, 9 \mathrm{H}, \mathrm{H}-15+\mathrm{H}-16$ + H-17). ${ }^{13} \mathrm{C}$ NMR (101 MHz, Chloroform-d) $\delta 173.8$ (C-9), 164.3 (C-2), 147.7 (C-6), 119.9 (C-1), 80.9 (C-7), 60.4 (C-4), 39.5 (C-5), 37.8 (C-13), 31.2 (C-18), 27.5 (C-15 + C-16 + C-17), 23.2 (C-12), 22.8 (C14). HRMS (EI+): $m / z$ calcd. for $\mathrm{C}_{14} \mathrm{H}_{22} \mathrm{~N}_{2} \mathrm{O}_{2} \mathrm{Na}[\mathrm{M}+\mathrm{Na}]^{+} 273.1573$, found 273.1580 .

(3S,8aS)-3-(tert-butyl)-2,7,8a-trimethyl-2,3,6,8a-tetrahydroimidazo[1,5-a]pyridine-1,5-dione 4h:<smiles>CC1=C[C@]2(C)C(=O)N(C)[C@H](C(C)(C)C)N2C(=O)C1</smiles><smiles>CC1=N[C@@]2(C)C(=O)N([13CH3])[C@H](C(C)(C)C)N2C(=O)C1</smiles>

Following GP2, the title compound $\mathbf{4 h}$ was prepared from $\mathbf{2 h}$ ( $43 \mathrm{mg}, 0.15 \mathrm{mmol}, 1.0$ equiv.). The reaction was finished after $2 \mathrm{~h}$. White solid ( $18 \mathrm{mg}, 0.07 \mathrm{mmol}, 47 \%) . \mathbf{m . p .}=90-94{ }^{\circ} \mathrm{C} . \boldsymbol{R}_{f} 0.60\left(\mathrm{SiO}_{2}\right.$; pet. Ether/ EtOAc 4/6). $[\alpha]_{\mathrm{D}}^{24}=-48^{\circ}\left(c=1\right.$ in $\left.\mathrm{CHCl}_{3}\right)$.

IR (neat, $\left.\mathbf{c m}^{-1}\right): V_{\max } 2979(\mathrm{C}-\mathrm{H}), 1705(\mathrm{C}=\mathrm{O}), 1679(\mathrm{C}=\mathrm{O}), 1264,782,768 .{ }^{1} \mathrm{H}$ NMR $(400 \mathrm{MHz}$, Chloroform-d) $\delta 5.84$ (ddd, $J=3.3,1.6,0.6 \mathrm{~Hz}, 1 \mathrm{H}, \mathrm{H}-5$ ), 5.15 (s, 1H, H-7), 3.09 (ddd, $J=18.2,3.3,1.6$ $\mathrm{Hz}, 1 \mathrm{H}, \mathrm{H}-1), 2.98(\mathrm{~s}, 3 \mathrm{H}, \mathrm{H}-18), 2.72(\mathrm{~d}, J=18.2 \mathrm{~Hz}, 1 \mathrm{H}, \mathrm{H}-1), 1.78(\mathrm{t}, J=1.5 \mathrm{~Hz}, 3 \mathrm{H}, \mathrm{H}-12), 1.50(\mathrm{~s}, 3 \mathrm{H}$, $\mathrm{H}-14), 1.07$ (s, 9H, H-15 + H-16 + H-17). ${ }^{13} \mathrm{C}$ NMR (101 MHz, Chloroform-d) $\delta 172.0$ (C-9), 171.0 (C-2), 130.5 (C-6), 124.6 (C-5), 80.8 (C-7), 64.3 (C-4), 38.2 (C-1), 37.2 (C-13), 31.2 (C-18), 27.4 (C-15 + C-16 + C-17), 24.9 (C-14), 21.7 (C-12). HRMS (EI+): $m / z$ calcd. for $\mathrm{C}_{14} \mathrm{H}_{23} \mathrm{~N}_{2} \mathrm{O}_{2}[\mathrm{M}+\mathrm{H}]^{+} 251.1754$, found 251.1758 . 
(3S,8aS)-3-(tert-butyl)-2,8a-dimethyl-7-methylenehexahydroimidazo[1,5-a]pyridine-1,5-dione 6h:<smiles>C=C1CC(=O)N2[C@@H](C(C)(C)C)N(C)C(=O)[C@]2(C)C1</smiles><smiles></smiles>

Following GP2, the title compound $\mathbf{6}$ h was prepared from $(2 \mathrm{~S}, 4 \mathrm{R})$-4-allyl-2-(tert-butyl)-1,4-dimethyl5-oxopyrrolidine-3-carbonyl chloride ( $43 \mathrm{mg}, 0.15 \mathrm{mmol}, 1.0$ equiv.). The reaction was finished after 2 h. Colourless oil ( $5 \mathrm{mg}, 0.02 \mathrm{mmol}, 13 \%) . \boldsymbol{R}_{f} 0.45\left(\mathrm{SiO}_{2}\right.$; pet. Ether/ EtOAc 4/6).

$[\alpha]_{\mathbf{D}}^{24}=-52^{\circ}\left(c=1\right.$ in $\left.\mathrm{CHCl}_{3}\right)$. IR (neat, $\left.\mathrm{cm}^{-1}\right): v_{\max } 2961(\mathrm{C}-\mathrm{H}), 1706(\mathrm{C}=\mathrm{O}), 1674(\mathrm{C}=\mathrm{O}), 1363$.

${ }^{1} \mathrm{H}$ NMR (400 MHz, Chloroform-d) $\delta 5.27(\mathrm{~s}, 1 \mathrm{H}, \mathrm{H}-7$ ), $5.11-4.97(\mathrm{~m}, 2 \mathrm{H}, \mathrm{H}-12), 3.30$ (dq, J = 20.2, 1.8 $\mathrm{Hz}, 1 \mathrm{H}, \mathrm{H}-1$ ), 3.20 (dq, J = 20.2, 2.1 Hz, 1H, H-1), 3.02 (s, 3H, H-18), 2.67 (d, J=13.9 Hz, 1H, H-5), 2.18 (d, J = $13.9 \mathrm{~Hz}, 1 \mathrm{H}, \mathrm{H}-5), 1.47(\mathrm{~s}, 3 \mathrm{H}, \mathrm{H}-14), 1.04(\mathrm{~s}, 9 \mathrm{H}, \mathrm{H}-15+\mathrm{H}-16+\mathrm{H}-17) .{ }^{13} \mathrm{C}$ NMR (101 MHz, Chloroform-d) $\delta 173.5$ (C-9), 170.7 (C-2), 135.9 (C-6), 113.9 (C-12), 80.7 (C-7), 61.4 (C-14), 43.0 (C-5), 38.4 (C-1), 37.5 (C-13), 31.2 (C-18), 27.2 (C-15 + C-16 + C-17), 22.6 (C-14). HRMS (EI+): $\mathrm{m} / \mathrm{z}$ calcd. for $\mathrm{C}_{14} \mathrm{H}_{22} \mathrm{~N}_{2} \mathrm{O}_{2} \mathrm{Na}[\mathrm{M}+\mathrm{Na}]^{+} 273.1573$, found 273.1573.

(3S,8aS)-3-(Tert-butyl)-2,8a-dimethyl-7-phenyl-2,3,8,8a-tetrahydroimidazo[1,5-a]pyridine-1,5dione $3 b$

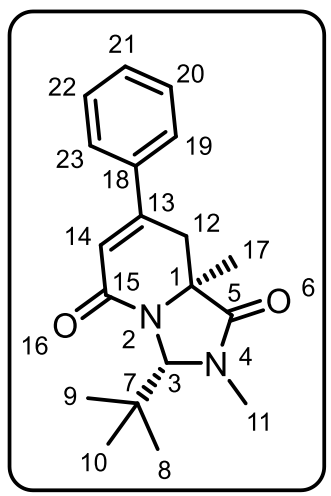<smiles></smiles>

and (3S,8aS)-3-(Tert-butyl)-2,8a-dimethyl-7-phenyl-2,3,6,8a-tetrahydroimidazo[1,5-a]pyridine-1,5dione $4 b$

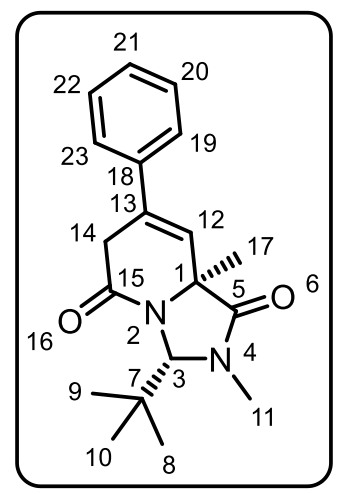<smiles>CCCCN1C(=O)N2C(=O)CC(c3cncnn3)=C[C@]2(C)C1C(C)(C)C</smiles> 
Following GP2, $N$-chloroformylimidazolidinone $\mathbf{2 b}(36.3 \mathrm{mg}, 0.105 \mathrm{mmol}, 1.0 \mathrm{eq}$.) was dissolved in dry MeCN $(0.53 \mathrm{~mL}, 0.2 \mathrm{M}$ ) before the addition of potassium iodide (19.2 mg, $0.116 \mathrm{mmol}, 1.1 \mathrm{eq}$.) and 2,6-lutidine ( $13.4 \mu \mathrm{L}, 0.116 \mathrm{mmol}, 1.1 \mathrm{eq}$.). The reaction was finished after $2 \mathrm{~h}$. The reaction was quenched with aqueous $\mathrm{HCl}(1.0 \mathrm{M})$. The reaction mixture was partitioned between $\mathrm{H}_{2} \mathrm{O}$ and EtOAc. The aqueous phase was further extracted with EtOAc (x2) and the combined organic phases were washed with brine, dried over $\mathrm{MgSO}_{4}$, filtered and the solvent was removed under reduced pressure. The residue was chromatographed $\left(\mathrm{SiO}_{2}\right.$, pet.ether/EtOAc, 4:6) to yield; $\mathbf{3 b}$ (9.5 mg, 29\%) as a yellow solid. $\mathbf{R}_{f}=0.36$ (pet.ether/EtOAc $\left.=4: 6\right) ;[\alpha]_{\mathrm{D}}^{23}=-164\left(c=1\right.$ in $\left.\mathrm{CHCl}_{3}\right) ; \mathbf{m p ~ 1 3 7 - 1 3 8}{ }^{\circ} \mathrm{C}$.

IR (neat, $\mathrm{cm}^{-1}$ ): $v_{\max }$ 2975, 2931 (C-H), 1705, 1661 (C=O); ${ }^{1} \mathbf{H}$ NMR (400 MHz, CDCl 3 ) $\delta 7.55-7.38(\mathrm{~m}$, $5 \mathrm{H}, \mathrm{H}-19+\mathrm{H}-20+\mathrm{H}-21+\mathrm{H}-22+\mathrm{H}-23), 6.32(\mathrm{~d}, \mathrm{~J}=2.8 \mathrm{~Hz}, 1 \mathrm{H}, \mathrm{H}-14), 5.26(\mathrm{~s}, 1 \mathrm{H}, \mathrm{H}-3), 3.07(\mathrm{~s}, 3 \mathrm{H}, \mathrm{H}-$ 11), $2.99(\mathrm{~d}, J=16.7 \mathrm{~Hz}, 1 \mathrm{H}, \mathrm{H}-12), 2.74(\mathrm{dd}, J=16.7,2.8 \mathrm{~Hz}, 1 \mathrm{H}, \mathrm{H}-12), 1.58(\mathrm{~s}, 2 \mathrm{H}, \mathrm{H}-17), 1.11$ (s, 9H, $\mathrm{H}-8+\mathrm{H}-9+\mathrm{H}-10) ;{ }^{13} \mathrm{C}$ NMR (101 MHz, CDCl 3 ) $\delta 173.92$ (C-5), 164.68 (C-15), 146.90 (C-18), 137.34 (C13), 130.00 (C-19 to 23), 129.06 (C-19 to 23), 126.08 (C-19 to 23), 119.08 (C-14), 81.19 (C-3), 60.77 (C-1), 38.02 (C-7), 37.03 (C-12), 31.51 (C-11), 27.64 (C-8 + C-9 + C-10), 22.85 (C-17); HRMS $\mathrm{C}_{19} \mathrm{H}_{24} \mathrm{~N}_{2} \mathrm{O}_{2}$ $[\mathrm{M}+\mathrm{H}]^{+}$calculated: 313.1911, found: 313.1924 .

and;

4b (15.8 mg, 48\%) as a yellow oil. $\mathbf{R}_{f}=0.50$ (pet.ether/EtOAc $\left.=4: 6\right) ;[\boldsymbol{\alpha}]_{\mathrm{D}}^{23}=-44\left(c=1\right.$ in $\left.\mathrm{CHCl}_{3}\right)$; IR (neat, $\left.\mathrm{cm}^{-1}\right): v_{\max }$ 2978, $2936(\mathrm{C}-\mathrm{H}), 1705,1674(\mathrm{C}=\mathrm{O})$; ${ }^{1} \mathrm{H}$ NMR $\left(400 \mathrm{MHz}, \mathrm{CDCl}_{3}\right) \delta 7.42-7.28(\mathrm{~m}, 5 \mathrm{H}$, $\mathrm{H}-19+\mathrm{H}-20+\mathrm{H}-21+\mathrm{H}-22+\mathrm{H}-23), 6.48(\mathrm{~d}, J=3.1 \mathrm{~Hz}, 1 \mathrm{H}, \mathrm{H}-12), 5.22(\mathrm{~s}, 1 \mathrm{H}, \mathrm{H}-3), 3.47$ (dd, J= 17.8, $3.1 \mathrm{~Hz}, 1 \mathrm{H}, \mathrm{H}-14), 3.37$ (d, J=17.8 Hz, 1H, H-14), 3.04 (s, 3H, H-11), 1.62 (s, 3H, H-17), 1.12 (s, 9H, H-8 + H-9 + H-10). ${ }^{13} \mathrm{C}$ NMR (101 MHz, CDCl ${ }_{3}$ ) $\delta 170.90$ (C-5), 137.68 (C-15), 133.07 (C-13), 128.82 (C-19 to 23), 128.46 (C-18), 125.96 (C-12), 125.48 (C-19 to 23), 81.06 (C-3), 64.86 (C-1), 37.45 (C-7), 36.09 (C-14), 31.52 (C-11), 27.60 (C-8 + C-9 + C-10), 24.85 (C-17); HRMS $\mathrm{C}_{19} \mathrm{H}_{24} \mathrm{~N}_{2} \mathrm{O}_{2}[\mathrm{M}+\mathrm{H}]^{+}$calculated: 313.1911, found: 313.1925.

Following GP3, $N$-chloroformylimidazolidinone $1 \mathbf{b}(21.7 \mathrm{mg}, 0.062 \mathrm{mmol}, 1.0$ eq.) was dissolved in dry $\operatorname{MeCN}(0.31 \mathrm{~mL}, 0.2 \mathrm{M}$ ) before the addition of potassium iodide $(11.4 \mathrm{mg}, 0.069 \mathrm{mmol}, 1.1 \mathrm{eq})$. and DBU $\left(28.0 \mu \mathrm{L}, 0.187 \mathrm{mmol}, 3.0\right.$ eq.). The reaction was heated to $150{ }^{\circ} \mathrm{C}$ in a microwave reactor for $2 \mathrm{~h}$. The reaction was quenched with aqueous $\mathrm{HCl}(1.0 \mathrm{M})$. The reaction mixture was partitioned between $\mathrm{H}_{2} \mathrm{O}$ and EtOAc. The aqueous phase was further extracted with EtOAc $\left(x_{2}\right)$ and the combined organic phases were washed with brine, dried over $\mathrm{MgSO}_{4}$, filtered and the solvent was removed under reduced pressure. The residue was chromatographed $\left(\mathrm{SiO}_{2}\right.$, pet.ether/EtOAc, 4:6) to yield $\mathbf{3 b}(9.2 \mathrm{mg}, 47 \%)$ as a yellow solid and $\mathbf{4 b}(2.6 \mathrm{mg}, 13 \%)$ as a white solid. Data in agreement with those which are reported above. 

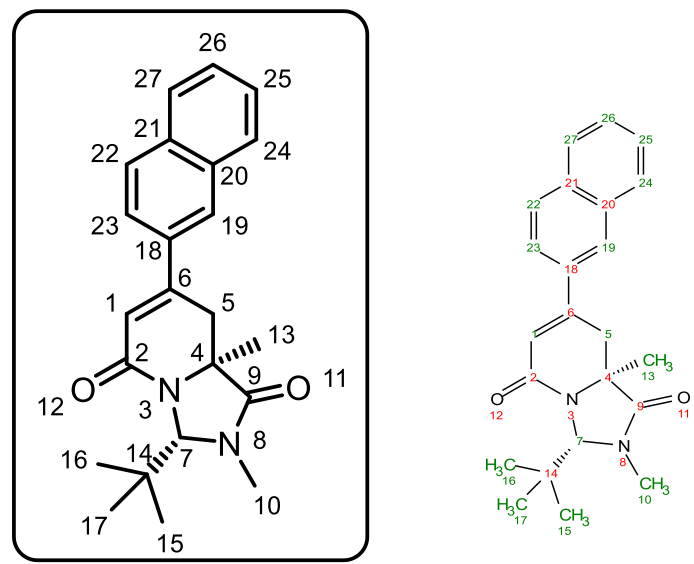

and (3S,8aS)-3-(Tert-butyl)-2,8a-dimethyl-7-(naphthalen-2-yl)-2,3,6,8a-tetrahydroimidazo[1,5a]pyridine-1,5-dione $4 f$
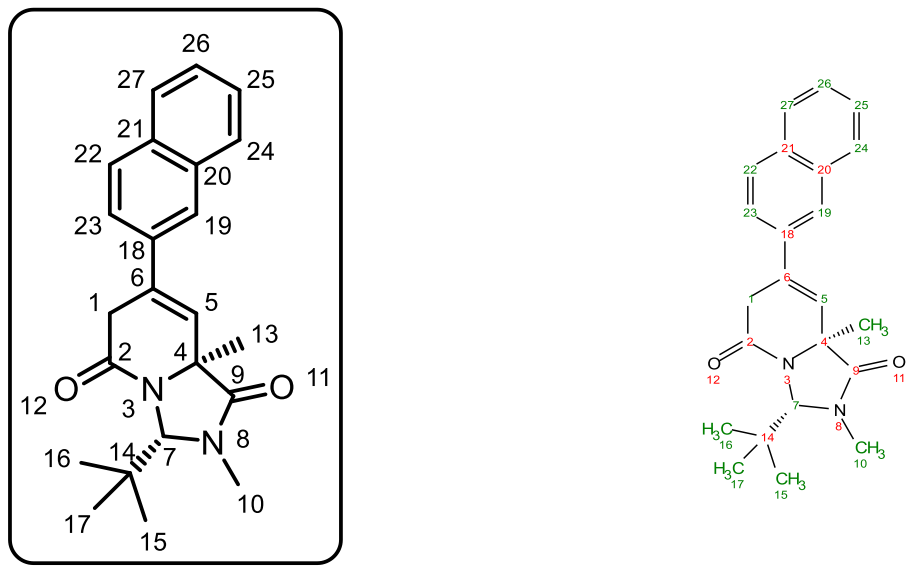

Following GP2, $N$-chloroformylimidazolidinone $2 \mathrm{f}(20.0 \mathrm{mg}, 0.050 \mathrm{mmol}, 1.0$ eq.) was dissolved in dry $\operatorname{MeCN}(0.25 \mathrm{~mL}, 0.2 \mathrm{M})$ before the addition of potassium iodide $(9.15 \mathrm{mg}, 0.116 \mathrm{mmol}, 1.1 \mathrm{eq}$. and 2,6-lutidine $\left(6.39 \mu \mathrm{L}, 0.055 \mathrm{mmol}, 1.1\right.$ eq.). The reaction was heated to $150{ }^{\circ} \mathrm{C}$ in a microwave reactor for $2 \mathrm{~h}$. The reaction was quenched with aqueous $\mathrm{HCl}(1.0 \mathrm{M})$. The reaction mixture was partitioned between $\mathrm{H}_{2} \mathrm{O}$ and EtOAc. The aqueous phase was further extracted with EtOAc $(\times 2)$ and the combined organic phases were washed with brine, dried over $\mathrm{MgSO}_{4}$, filtered and the solvent was removed under reduced pressure. The residue was chromatographed $\left(\mathrm{SiO}_{2}\right.$, pet.ether/EtOAc, $4: 6)$ to yield;

3f $(8.0 \mathrm{mg}, 44 \%)$ as a white solid. $\mathbf{R}_{f}=0.28$ (pet.ether/EtOAc $\left.=4: 6\right) ;[\boldsymbol{\alpha}]_{\mathrm{D}}^{23}=-172\left(c=1\right.$ in $\left.\mathrm{CHCl}_{3}\right)$; $\mathbf{m p}$ 146-147 ${ }^{\circ} \mathrm{C}$; IR (neat, $\mathrm{cm}^{-1}$ ): $\mathrm{v}_{\max }$ 2962, 2925 (C-H), 1701, 1655 (C=O); ${ }^{1} \mathbf{H}$ NMR (400 MHz, CDCl 3 ) $\delta$ $7.96(\mathrm{~d}, J=1.8 \mathrm{~Hz}, 1 \mathrm{H}, \mathrm{H}-19), 7.86(\mathrm{td}, J=8.0,6.2,3.5 \mathrm{~Hz}, 3 \mathrm{H}, \mathrm{H}-22+\mathrm{H}-24+\mathrm{H}-27), 7.66$ (dd, $J=8.0$, $1.8 \mathrm{~Hz}, 1 \mathrm{H}, \mathrm{H}-23), 7.53(\mathrm{dt}, J=6.2,3.5 \mathrm{~Hz}, 2 \mathrm{H}, \mathrm{H}-25+\mathrm{H}-26), 6.47$ (d, J = 2.7 Hz, 1H, H-1), $5.29(\mathrm{~s}, 1 \mathrm{H}$, H-7), 3.17 (d, J = 16.5 Hz, 1H, H-5), 3.09 (s, 3H, H-10), 2.83 (dd, J=16.5, 2.7 Hz, 1H, H-5), 1.66 (s, 3H, $\mathrm{H}-13), 1.13$ (s, 9H, H-15 + H-16 + H-17); ${ }^{13} \mathrm{C}$ NMR (101 MHz, CDCl $)$ ) 174.01 (C-9), 164.76 (C-2), 
146.50 (C-6), 134.32 (C-18 to C-27), 134.00 (C-18 to C-27), 133.27 (C-18 to C-27), 128.84 (C-18 to C27), 128.78 (C-18 to C-2), 127.81 (C-18 to C-27), 127.40 (C-18 to C-27), 126.99 (C-18 to C-27), 126.20 (C-18 to C-27), 123.11 (C-18 to C-27), 119.24 (C-1), 81.26 (C-7), 60.81 (C-4), 38.04 (C-14), 36.93 (c-5), 31.55 (C-10), 27.65 (C-15 + C-16 + C-17), 22.90 (C-13); HRMS $\mathrm{C}_{23} \mathrm{H}_{26} \mathrm{~N}_{2} \mathrm{O}_{2}[\mathrm{M}+\mathrm{H}]^{+}$calculated: 363.2067, found: 363.2074 .

4f $(10.5 \mathrm{mg}, 58 \%)$ as a white semi-solid. $\mathbf{R}_{\boldsymbol{f}}=0.49$ (pet.ether/EtOAc $\left.=4: 6\right) ;[\boldsymbol{\alpha}]_{\mathrm{D}}^{24}=-36\left(c=1\right.$ in $\left.\mathrm{CHCl}_{3}\right)$; IR (neat, cm $\left.{ }^{-1}\right)$ : $v_{\max } 2974,2926(\mathrm{C}-\mathrm{H}), 1707,1675$ (C=O); ${ }^{1} \mathbf{H}$ NMR (400 MHz, CDCl $) \delta 7.87-7.75(\mathrm{~m}$, $4 \mathrm{H}, \mathrm{H}-19+\mathrm{H}-22+\mathrm{H}-24+\mathrm{H}-27), 7.60-7.44(\mathrm{~m}, 3 \mathrm{H}, \mathrm{H}-23+\mathrm{H}-25+\mathrm{H}-26), 6.63(\mathrm{~d}, J=1.9 \mathrm{~Hz}, 1 \mathrm{H}, \mathrm{H}-5)$, $5.25(\mathrm{~s}, 1 \mathrm{H}, \mathrm{H}-7), 3.55$ (d, J = 2.6 Hz, 2H, H-1), 3.05 (s, 3H, H-10), 1.67 (s, 3H, H-13), 1.14 (s, 9H, H-15 + $\mathrm{H}-16+\mathrm{H}-17) ;{ }^{13} \mathrm{C} \mathrm{NMR}\left(101 \mathrm{MHz}, \mathrm{CDCl}_{3}\right.$ ) $\delta 134.80$ (C-9), 133.39 (C-2), 133.22 (C-6), 132.92 (C-18 to C27), 128.54 (C-18 to C-27), 128.39 (C-18 to C-27), 127.74 (C-18 to C-27), 126.69 (C-18 to C-27), 126.57 (C-18 to C-27), 126.39 (C-5), 124.53 (C-18 to C-27), 123.37 (C-18 to C-27), 81.12 (C-7), 64.95 (C-14), 37.47 (C-14), 36.03 (C-1), 31.54 (C-10), 27.62 (C-15 + C-16 + C-17), 24.91 (C-13); HRMS $\mathrm{C}_{23} \mathrm{H}_{26} \mathrm{~N}_{2} \mathrm{O}_{2}[\mathrm{M}+\mathrm{H}]^{+}$calculated: 363.2067 , found: 363.2076 .

Following GP3, $N$-chloroformylimidazolidinone $\mathbf{4 f}(20.0 \mathrm{mg}, 0.050 \mathrm{mmol}, 1.0$ eq.) was dissolved in dry $\operatorname{MeCN}(0.25 \mathrm{~mL}, 0.2 \mathrm{M}$ ) before the addition of potassium iodide $(9.15 \mathrm{mg}, 0.055 \mathrm{mmol}, 1.1 \mathrm{eq}$.) and $\mathrm{DBU}\left(22.5 \mu \mathrm{L}, 0.150 \mathrm{mmol}, 3.0\right.$ eq.). The reaction was heated to $150{ }^{\circ} \mathrm{C}$ in a microwave reactor for $2 \mathrm{~h}$. The reaction was quenched with aqueous $\mathrm{HCl}(1.0 \mathrm{M})$. The reaction mixture was partitioned between $\mathrm{H}_{2} \mathrm{O}$ and EtOAc. The aqueous phase was further extracted with EtOAc $\left(x_{2}\right)$ and the combined organic phases were washed with brine, dried over $\mathrm{MgSO}_{4}$, filtered and the solvent was removed under reduced pressure. The residue was chromatographed $\left(\mathrm{SiO}_{2}\right.$, pet.ether/EtOAc, 4:6) to yield 147 (13.6 mg, 75\%) as a yellow solid and $\mathbf{3 f}(2.4 \mathrm{mg}, 13 \%)$ as a white solid. Data in agreement with those which are reported above.

(3S,8aS)-3-(tert-butyl)-7-(3,4-dichlorophenyl)-2,8a-dimethyl-2,3,6,8a-tetrahydroimidazo[1,5a]pyridine-1,5-dione $4 \mathrm{~d}$ :
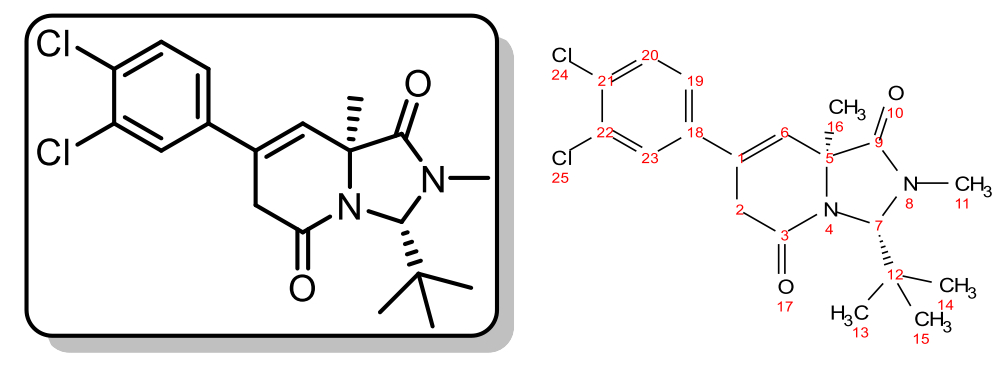

Following GP2, the title compound $\mathbf{4 d}$ was prepared from $\mathbf{2 d}(151 \mathrm{mg}, 0.36 \mathrm{mmol}, 1.0$ equiv.) according to the general procedure. The reaction was finished after $2 \mathrm{~h}$. Colourless oil (43 $\mathrm{mg}, 31 \%$ ). $\mathbf{R}_{\mathbf{f}} 0.21\left(\mathrm{SiO}_{2}\right.$; pet. Ether/ EtOAc 4:1). $[\boldsymbol{\alpha}]_{\mathrm{D}}^{26}=-20^{\circ}\left(c=1\right.$ in $\left.\mathrm{CHCl}_{3}\right)$.

IR (neat, cm ${ }^{-1}$ ): $v_{\max } 2970(\mathrm{C}-\mathrm{H}), 1708$ (C=O), 1674 (C=O), 1605; ${ }^{1} \mathrm{H}$ NMR (500 MHz, CDCl $\left.)_{3}\right) \delta 7.47$ (d, J $=2.2 \mathrm{~Hz}, 1 \mathrm{H}, \mathrm{H}-\mathrm{Ar}$ ), 7.42 (d, J = 8.4 Hz, 1H, H-Ar), 7.20 (dd, J = 8.4, $2.2 \mathrm{~Hz}, 1 \mathrm{H}, \mathrm{H}-\mathrm{Ar}$ ), 6.49 (dd, J = 3.3, $0.8 \mathrm{~Hz}, 1 \mathrm{H}, \mathrm{H}-6), 5.21$ (s, $1 \mathrm{H}, \mathrm{H}-7$ ), 3.43 (dd, J=17.7, $3.3 \mathrm{~Hz}, 1 \mathrm{H}, \mathrm{H}-2$ ), 3.28 (dd, J=17.8, $0.7 \mathrm{~Hz}, 1 \mathrm{H}, \mathrm{H}-$ 2), $3.03(\mathrm{~s}, 3 \mathrm{H}, \mathrm{H}-11), 1.62(\mathrm{~s}, 3 \mathrm{H}, \mathrm{H}-16), 1.12(\mathrm{~s}, 9 \mathrm{H}, \mathrm{H}-13, \mathrm{H}-14, \mathrm{H}-15) .{ }^{13} \mathrm{C} \mathrm{NMR}\left(126 \mathrm{MHz}, \mathrm{CDCl}_{3}\right) \delta$ 171.3 (C-9), 170.2 (C-3), 137.6 (C-Ar), 133.1 (C-Ar), 132.5 (C-Ar), 131.2 (C-Ar), 130.7 (CH-Ar), 127.6 (CH-Ar), 127.5 (CH-Ar), 124.7 (CH-Ar), 81.2 (C-7), 64.8 (C-5), 37.4 (C-12), 35.8 (C-2), 31.5 (C-11), 27.6 (C-13, C-14, C-15), 24.8 (C-16). HRMS $\mathrm{C}_{19} \mathrm{H}_{22} \mathrm{Cl}_{2} \mathrm{~N}_{2} \mathrm{O}_{2}[\mathrm{M}+\mathrm{H}]^{+}$calculated: 381.1131, found 381.1138. 
(3S,8aS)-3-(tert-butyl)-7-(3,4-dichlorophenyl)-2,8a-dimethyl-2,3,8,8a-tetrahydroimidazo[1,5a]pyridine-1,5-dione $3 \mathrm{~d}$ :
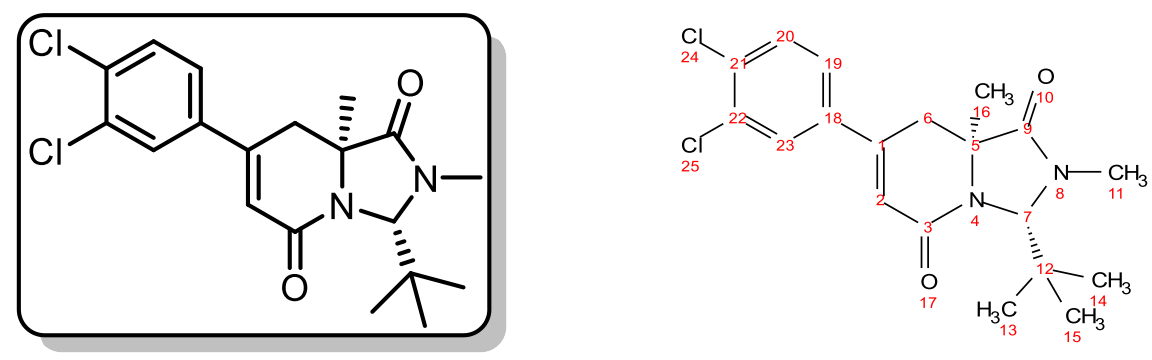

Following GP2, the title compound $\mathbf{3} \mathbf{d}$ was prepared from $\mathbf{2 d}(151 \mathrm{mg}, 0.48 \mathrm{mmol}, 1.0$ equiv.). The reaction was finished after $2 \mathrm{~h}$. Colourless oil (73 mg, 53\%). $\mathbf{R}_{f} 0.09$ ( $\mathrm{SiO}_{2}$; pet. Ether/ EtOAc 4:1).

$[\alpha]_{\mathrm{D}}^{26}=-192^{\circ}\left(c=1\right.$ in $\left.\mathrm{CHCl}_{3}\right)$. IR (neat, $\left.\mathrm{cm}^{-1}\right): v_{\max } 2972(\mathrm{C}-\mathrm{H}), 1703(\mathrm{C}=\mathrm{O}), 1671(\mathrm{C}=\mathrm{O}), 1605$.

${ }^{1} \mathrm{H}$ NMR $\left(500 \mathrm{MHz}, \mathrm{CDCl}_{3}\right) \delta 7.57$ (d, $J=2.2 \mathrm{~Hz}, 1 \mathrm{H}, \mathrm{H}-\mathrm{Ar}$ ), 7.48 (d, $J=8.5 \mathrm{~Hz}, 1 \mathrm{H}, \mathrm{H}-\mathrm{Ar}$ ), 7.33 (dd, $J=$ 8.5, $2.2 \mathrm{~Hz}, 1 \mathrm{H}, \mathrm{H}-\mathrm{Ar}$ ), 6.30 (d, J = $2.7 \mathrm{~Hz}, 1 \mathrm{H}, \mathrm{H}-2), 5.24(\mathrm{~s}, 1 \mathrm{H}, \mathrm{H}-7), 3.06(\mathrm{~s}, 3 \mathrm{H}, \mathrm{H}-11), 2.88$ (d, $J=$ $16.6 \mathrm{~Hz}, 1 \mathrm{H}, \mathrm{H}-6$ ), 2.71 (dd, J = 16.6, 2.8 Hz, 1H, H-6), 1.60 (s, 3H, H-16), 1.10 (s, 9H, H-13, H-14, H15). ${ }^{13} \mathrm{C}$ NMR (126 MHz, CDCl ${ }_{3}$ ) $\delta 173.4$ (C-3), 163.9 (C-9), 144.3 (C-Ar), 137.1 (C-Ar), 134.0 (C-Ar), 133.4 (C-Ar), 130.9 (CH-Ar), 127.9 (CH-Ar), 125.0 (CH-Ar), 120.2 (C-2), 81.1 (C-7), 60.5 (C-5), 37.9 (C12), 36.7 (C-6) , 31.4 (C-11), 27.5 (C-13, C-14, C-15), 22.7 (C-16). HRMS $\mathrm{C}_{19} \mathrm{H}_{22} \mathrm{Cl}_{2} \mathrm{~N}_{2} \mathrm{O}_{2}[\mathrm{M}+\mathrm{H}]^{+}$ calculated: 381.1131 , found 381.1131 .

\section{Isomerisation of $4 \mathrm{~d}$ to $3 \mathrm{~d}$}

Following GP4, 4d (92.0 mg, $0.29 \mathrm{mmol}, 1.0$ eq.) was dissolved in dry MeCN (1.14 mL, $0.25 \mathrm{M})$ before the addition of DBU (130 $\mu \mathrm{L}, 0.72 \mathrm{mmol}, 3.0$ eq.). The reaction was heated to $150{ }^{\circ} \mathrm{C}$ in a microwave reactor for $2 \mathrm{~h}$. The reaction was quenched with aqueous $\mathrm{HCl}(1.0 \mathrm{M})$. The reaction mixture was partitioned between $\mathrm{H}_{2} \mathrm{O}$ and EtOAc. The aqueous phase was further extracted with EtOAc (x2) and the combined organic phases were washed with brine, dried over $\mathrm{MgSO}_{4}$, filtered and the solvent was removed under reduced pressure. The crude product was purified by flash column chromatography $\left(\mathrm{SiO}_{2}\right.$, pet.ether/EtOAc, 4:6) to yield $\mathbf{3 d}(69 \mathrm{mg}, 75 \%)$ as a white solid. Data in agreement with those which are reported above. 

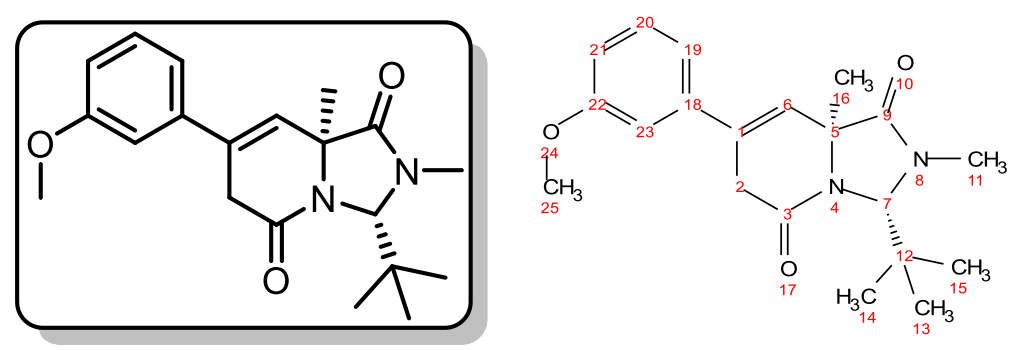

Following GP2, the title compound $\mathbf{4 g}$ was prepared from $\mathbf{2 d}(132 \mathrm{mg}, 0.35 \mathrm{mmol}, 1.0$ equiv.). The reaction was finished after $2 \mathrm{~h}$. Colourless oil (64 mg, 53\%). $\mathbf{R}_{\mathbf{f}} 0.07$ ( $\mathrm{SiO}_{2}$; pet. Ether/ EtOAc 4:1).

$[\alpha]_{\mathrm{D}}^{26}=-24^{\circ}\left(c=1\right.$ in $\left.\mathrm{CHCl}_{3}\right)$. IR (neat, $\left.\mathrm{cm}^{-1}\right): v_{\max } 2968(\mathrm{C}-\mathrm{H}), 1702(\mathrm{C}=\mathrm{O}), 1680(\mathrm{C}=\mathrm{O}), 1610$.

${ }^{1} \mathrm{H}$ NMR $\left(500 \mathrm{MHz}, \mathrm{CDCl}_{3}\right) \delta 7.32(\mathrm{t}, J=8.0 \mathrm{~Hz}, 1 \mathrm{H}, \mathrm{H}-20), 7.08$ (dd, J = 7.7, $\left.0.9 \mathrm{~Hz}, 1 \mathrm{H}, \mathrm{H}-23\right), 7.00$ (t, J $=2.1 \mathrm{~Hz}, 1 \mathrm{H}, \mathrm{H}-21), 6.93(\mathrm{dd}, J=7.8,2.1 \mathrm{~Hz}, 1 \mathrm{H}, \mathrm{H}-19), 6.30$ (d, J=2.7 Hz, 1H, H-6), $5.24(\mathrm{~s}, 1 \mathrm{H}, \mathrm{H}-7$ ), $3.82(\mathrm{~s}, 3 \mathrm{H}, \mathrm{H}-25), 3.05$ (s, 3H, H-11), 2.95 (d, J = 16.6 Hz, 1H, H-2), $2.72(\mathrm{dt}, J=16.6,1.7 \mathrm{~Hz}, 1 \mathrm{H}, \mathrm{H}-2)$, 1.60 (s, 3H, H-16), 1.10 (s, 9H, H-13, H-14, H-15). ${ }^{13} \mathrm{C}$ NMR (126 MHz, CDCl ${ }_{3}$ ) $\delta 173.8$ (C-9), 164.6 (C3), 160.0 (C-Ar), 146.8 (C-Ar), 138.8 (C-1), 130.0 (C-20), 119.2 (C-6), 118.5 (C-23), 115.6 (C-19), 111.5 (C-21), 81.1 (C-7), 60.7 (C-5), 55.5 (C-25), 38.0 (C-12), 37.1 (C-2), 31.5 (C-11), 27.6 (C-13, C-14, C-15), 22.8 (C-16). HRMS $\mathrm{C}_{20} \mathrm{H}_{26} \mathrm{~N}_{2} \mathrm{O}_{3}[\mathrm{M}+\mathrm{H}]^{+}$calculated: 343.2016, found 343.2014.

(3S,8aS)-3-(tert-butyl)-7-(3-methoxyphenyl)-2,8a-dimethyl-2,3,8,8a-tetrahydroimidazo[1,5a]pyridine-1,5-dione 3g:
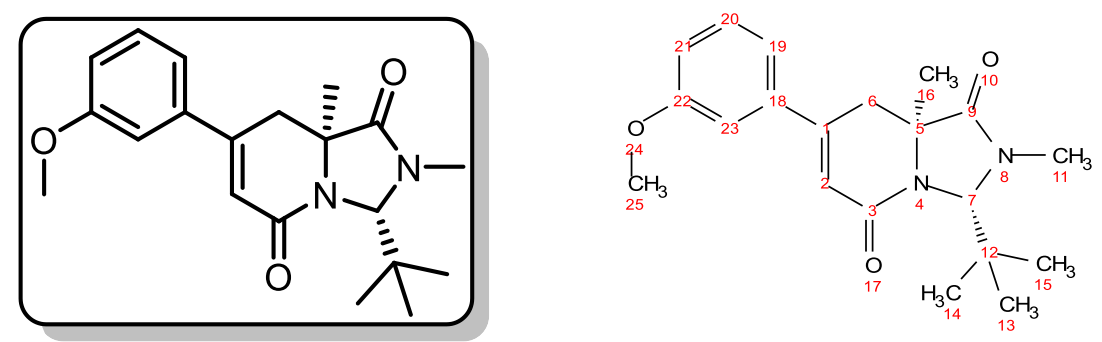

Following GP2, the title compound $\mathbf{3 g}$ was prepared from $\mathbf{2 g}(132 \mathrm{mg}, 0.35 \mathrm{mmol}, 1.0$ equiv.). The reaction was finished after $2 \mathrm{~h}$. Colourless oil $(50 \mathrm{mg}, 0.02 \mathrm{mmol}, 42 \%) . \mathbf{R}_{\mathbf{f}} 0.14$ ( $\mathrm{SiO}_{2}$; pet. Ether/ EtOAc 4:1).

$[\alpha]_{\mathrm{D}}^{26}=-144^{\circ}\left(c=1\right.$ in $\left.\mathrm{CHCl}_{3}\right)$. IR (neat, $\left.\mathrm{cm}^{-1}\right): v_{\max } 2978(\mathrm{C}-\mathrm{H}), 1709(\mathrm{C}=\mathrm{O}), 1668(\mathrm{C}=0), 1604$.

${ }^{1} \mathrm{H}$ NMR $\left(500 \mathrm{MHz}, \mathrm{CDCl}_{3}\right) \delta 7.26(\mathrm{t}, J=8.0 \mathrm{~Hz}, 1 \mathrm{H}, \mathrm{H}-20), 6.96$ (ddd, $J=7.7,1.7,0.9 \mathrm{~Hz}, 1 \mathrm{H}, \mathrm{H}-23$ ), $6.89(\mathrm{t}, J=2.1 \mathrm{~Hz}, 1 \mathrm{H}, \mathrm{H}-21$ ), 6.84 (ddd, $J=8.0,2.6,0.9 \mathrm{~Hz}, 1 \mathrm{H}, \mathrm{H}-19), 6.46$ (d, $J=3.7 \mathrm{~Hz}, 1 \mathrm{H}, \mathrm{H}-2$ ), 5.21 (s, 1H, H-7), 3.80 (s, 3H, H-25), 3.45 (dd, J=17.8, 3.3 Hz, 1H, H-6), 3.35 (dd, $J=17.8,3.3 \mathrm{~Hz}, 1 \mathrm{H}$, $\mathrm{H}-6), 3.02$ (s, 3H, H-11), 1.61 (s, 3H, H-16), 1.11 (s, 9H, H-13, H-14, H-15). ${ }^{13} \mathrm{C}$ NMR (126 MHz, CDCl 3 ) $\delta$ 171.7 (C-3), 170.8 (C-9), 159.9 (C-22), 139.1 (C-1), 132.9 (C-18), 129.8 (C-20), 126.1 (C-2), 117.9 (C23), 114.1 (C-19), 110.9 (C-21), 81.0 (C-7), 64.8 (C-5), 55.4 (C-25), 37.4 (C-12), 36.1 (C-6), 31.5 (C-11), 27.5 (C-13, C-14, C-15), 24.8 (C-16). HRMS $\mathrm{C}_{20} \mathrm{H}_{26} \mathrm{~N}_{2} \mathrm{O}_{3}[\mathrm{M}+\mathrm{H}]^{+}$calculated: 343.2016, found 343.2013. 


\section{Isomerisation of $4 \mathrm{~g}$ to $3 \mathrm{~g}$}

Following GP4, 4d (63.0 mg, $0.30 \mathrm{mmol}, 1.0$ eq.) was dissolved in dry MeCN (1.20 mL, $0.25 \mathrm{M})$ before the addition of DBU (130 $\mu \mathrm{L}, 0.73 \mathrm{mmol}, 3.0$ eq.). The reaction was heated to $150{ }^{\circ} \mathrm{C}$ in a microwave reactor for $2 \mathrm{~h}$. The reaction was quenched with aqueous $\mathrm{HCl}(1.0 \mathrm{M})$. The reaction mixture was partitioned between $\mathrm{H}_{2} \mathrm{O}$ and EtOAc. The aqueous phase was further extracted with EtOAc (x2) and the combined organic phases were washed with brine, dried over $\mathrm{MgSO}_{4}$, filtered and the solvent was removed under reduced pressure. The crude product was purified by flash column chromatography $\left(\mathrm{SiO}_{2}\right.$, pet.ether/EtOAc, 4:6) to yield $\mathbf{3 g}(55.44 \mathrm{mg}, 88 \%)$ as a white solid. Data in agreement with those which are reported above.

(3S,8aS)-3-(tert-butyl)-7-(4-fluorophenyl)-2,8a-dimethyl-2,3,8,8a-tetrahydroimidazo[1,5a]pyridine-1,5-dione $3 \mathrm{c}$ :
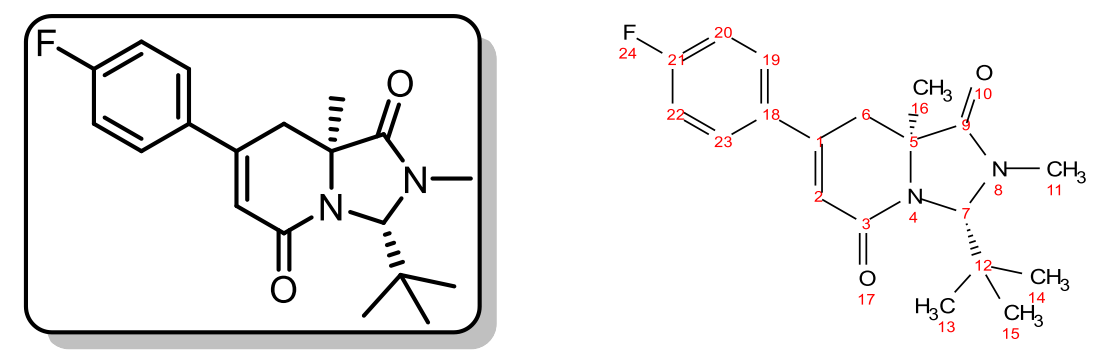

Following GP2, the title compound 3c was prepared from 2c (175 mg, $0.48 \mathrm{mmol}, 1.0$ equiv.) according to the general procedure. The reaction was finished after $2 \mathrm{~h}$. Colourless oil (39 mg, 25\%). $\mathbf{R}_{\mathbf{f}} 0.13\left(\mathrm{SiO}_{2}\right.$; pet. Ether/ EtOAc 4:1). $[\boldsymbol{\alpha}]_{\mathbf{D}}^{26}=-28^{\circ}\left(c=1\right.$ in $\left.\mathrm{CHCl}_{3}\right)$. IR (neat, $\left.\mathbf{c m}^{-1}\right): v_{\max } 2962(\mathrm{C}-\mathrm{H})$,

1705 (C=O), 1670 (C=O), 1600; ${ }^{1} \mathrm{H}$ NMR (500 MHz, CDCl $)$ $\delta 7.51-7.46(\mathrm{~m}, 2 \mathrm{H}, \mathrm{H}-19, \mathrm{H}-23), 7.10$ (dd, $J=8.9,8.3 \mathrm{~Hz}, 2 \mathrm{H}, \mathrm{H}-20, \mathrm{H}-22), 6.25(\mathrm{~d}, J=2.7 \mathrm{~Hz}, 1 \mathrm{H}, \mathrm{H}-2), 5.24(\mathrm{~s}, 1 \mathrm{H}, \mathrm{H}-7), 3.06(\mathrm{~s}, 3 \mathrm{H}, \mathrm{H}-11), 2.93$ (d, J = 16.5 Hz, 1H, H-6), 2.71 (ddd, $J=16.6,2.8,0.9 \mathrm{~Hz}, 1 \mathrm{H}, \mathrm{H}-6$ ), 1.60 (d, $J=0.8 \mathrm{~Hz}, 3 \mathrm{H}, \mathrm{H}-16$ ), 1.10 (s, 9H, H-13, H-14, H-15). ${ }^{13} \mathrm{C}$ NMR (126 MHz, CDCl $)$ $\delta 173.8$ (C-3), 164.8 (C-21), 164.5 (C-9), 162.8 (C21), 145.7 (C-1), 133.4 (C-18), 133.4 (C-18), 128.0 (C-19, C-23), 127.9 (C-19, C-23), 118.9 (C-2), 118.8 (C-2), 116.2 (C-20, C-21), 116.0 (C-20, C-21), 81.2 (C-7), 60.7 (C-5), 38.0 (C-12), 37.1 (C-6), 31.5 (C-11), 27.6 (C-13, C-14, C-15), 22.8 (C-16). HRMS $\mathrm{C}_{19} \mathrm{H}_{23} \mathrm{FN}_{2} \mathrm{O}_{2}[\mathrm{M}+\mathrm{H}]^{+}$calculated: 331.1816, found 331.1815.

(3S,8aS)-3-(tert-butyl)-7-(4-fluorophenyl)-2,8a-dimethyl-2,3,6,8a-tetrahydroimidazo[1,5a]pyridine-1,5-dione $4 \mathrm{c}$ :
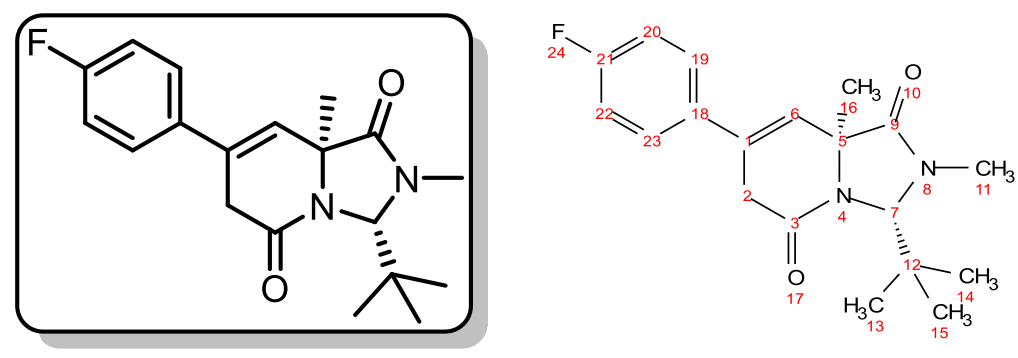

Following GP2, the title compound $4 \mathrm{c}$ was prepared from $2 \mathrm{c}(175 \mathrm{mg}, 0.48 \mathrm{mmol}, 1.0$ equiv.). The reaction was finished after $2 \mathrm{~h}$. Colourless oil $(63 \mathrm{mg}, 40 \%) . \mathbf{R}_{\mathbf{f}} 0.08$ ( $\mathrm{SiO}_{2}$; pet. Ether/ EtOAc 4:1). $[\alpha]_{\mathrm{D}}^{26}=-176^{\circ}\left(c=1\right.$ in $\left.\mathrm{CHCl}_{3}\right)$. IR (neat, $\left.\mathrm{cm}^{-1}\right): v_{\max } 2958(\mathrm{C}-\mathrm{H}), 1702(\mathrm{C}=\mathrm{O}), 1667(\mathrm{C}=\mathrm{O}), 1613$. 
${ }^{1} \mathrm{H}$ NMR $\left(500 \mathrm{MHz}, \mathrm{CDCl}_{3}\right) \delta 7.37-7.31(\mathrm{~m}, 2 \mathrm{H}, \mathrm{H}-19, \mathrm{H}-23), 7.03(\mathrm{t}, J=8.6 \mathrm{~Hz}, 2 \mathrm{H}, \mathrm{H}-20, \mathrm{H}-23), 6.40$ (d, $J=3.3 \mathrm{~Hz}, 1 \mathrm{H}, \mathrm{H}-6), 5.20$ (s, 1H, H-7), 3.44 (dd, J=17.7, $3.3 \mathrm{~Hz}, 1 \mathrm{H}, \mathrm{H}-2$ ), 3.31 (d, J=17.7 Hz, $1 \mathrm{H}$, $\mathrm{H}-2$ ), 3.02 (s, 3H, H-11), 1.61 (s, 3H, H-16), 1.11 (s, 9H, H-13, H-14, H-15). ${ }^{13} \mathrm{C} \mathrm{NMR}\left(126 \mathrm{MHz}, \mathrm{CDCl}_{3}\right) \delta$ 171.6 (C-9), 170.6 (C-9), 163.8 (C-3), 161.8 (C-3), 133.8 (C-21), 133.7 (C-21), 132.1 (C-1), 127.2 (C-20, C-22), 127.1 (C-20, C-22), 125.8 (C-18), 125.7 (C-18), 115.8 (C-19, C-23), 115.6 (C-19, C-23), 81.0 (C-7), 64.8 (C-5), 37.4 (C-12), 36.2 (C-2), 31.5 (C-11), 27.5 (C-13, C-14, C-15), 24.8 (C-16). HRMS $\mathrm{C}_{19} \mathrm{H}_{23} \mathrm{FN}_{2} \mathrm{O}_{2}$ $[\mathrm{M}+\mathrm{H}]^{+}$calculated: 331.1816 , found 331.1813 .

\section{Isomerisation of $4 \mathrm{c}$ to $3 \mathrm{c}$}

Following GP4, 4c (73.0 mg, $0.22 \mathrm{mmol}, 1.0$ eq.) was dissolved in dry MeCN ( $0.88 \mathrm{~mL}, 0.25 \mathrm{M}$ ) before the addition of $\mathrm{DBU}\left(100 \mu \mathrm{L}, 0.66 \mathrm{mmol}, 3.0 \mathrm{eq}\right.$.). The reaction was heated to $150^{\circ} \mathrm{C}$ in a microwave reactor for $2 \mathrm{~h}$. The reaction was quenched with aqueous $\mathrm{HCl}(1.0 \mathrm{M})$. The reaction mixture was partitioned between $\mathrm{H}_{2} \mathrm{O}$ and EtOAc. The aqueous phase was further extracted with EtOAc $(\mathrm{x} 2)$ and the combined organic phases were washed with brine, dried over $\mathrm{MgSO}_{4}$, filtered and the solvent was removed under reduced pressure. The crude product was purified by flash column chromatography $\left(\mathrm{SiO}_{2}\right.$, pet.ether/EtOAc, 4:6) to yield $3 \mathrm{c}(57.0 \mathrm{mg}, 78 \%)$ as a white solid. Data in agreement with those which are reported above.

(3S,8aS)-7-(4'-bromo-[1,1'-biphenyl]-4-yl)-3-(tert-butyl)-2,8a-dimethyl-2,3,6,8atetrahydroimidazo[1,5-a]pyridine-1,5-dione 4e:
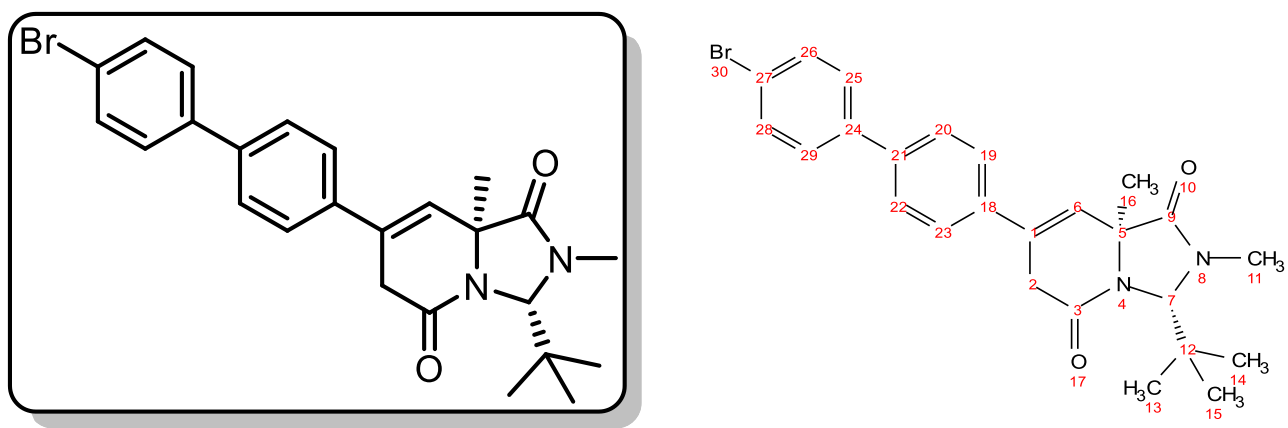

Following GP2, the title compound $4 \mathrm{e}$ was prepared from $2 \mathrm{e}(274 \mathrm{mg}, 0.54 \mathrm{mmol}, 1.0$ equiv.). The reaction was finished after $2 \mathrm{~h}$. Colourless oil (101 mg, 40\%). $\mathbf{R}_{f} 0.09$ ( $\mathrm{SiO}_{2}$; pet. Ether/ EtOAc 4:1). $[\alpha]_{\mathrm{D}}^{26}=+8^{\circ}\left(c=1\right.$ in $\left.\mathrm{CHCl}_{3}\right)$.

IR (neat, cm ${ }^{-1}$ ): $v_{\max } 2965(\mathrm{C}-\mathrm{H}), 1710(\mathrm{C}=\mathrm{O}), 1679$ (C=O), 1604; ${ }^{1} \mathrm{H}$ NMR (500 MHz, CDCl $) \delta 7.53$ (dd, $J=8.5,1.8 \mathrm{~Hz}, 4 \mathrm{H}, \mathrm{H}-\mathrm{Ar}), 7.47-7.39(\mathrm{~m}, 4 \mathrm{H}, \mathrm{H}-\mathrm{Ar}), 6.52(\mathrm{~d}, J=3.1 \mathrm{~Hz}, 1 \mathrm{H}, \mathrm{H}-6), 5.22(\mathrm{~s}, 1 \mathrm{H}, \mathrm{H}-7), 3.48$ (dd, J = 17.7, 3.2 Hz, 1H, H-2), 3.39 (d, J = 17.7 Hz, 1H, H-2), 3.03 (s, 3H, H-11), $1.63(\mathrm{~s}, 3 \mathrm{H}, \mathrm{H}-16), 1.11$ (s, 9H, H-13, H-14, H-15). ${ }^{13} \mathrm{C}$ NMR (126 MHz, CDCl $)$ ) 171.5 (C-9), 170.6 (C-3), 139.8 (C-Ar), 139.2 (CAr), 136.8 (C-1), 132.4 (C-Ar), 131.9 (CH-Ar), 128.5 (CH-Ar), 127.1 (CH-Ar), 126.0 (C-Ar), 125.9 (CHAr), 121.8 (C-Ar), 80.9 (C-7), 64.7 (C-5), 37.3 (C-12), 35.8 (C-6), 31.4 (C-11), 27.5 (C-13, C-14, C-15), 24.7 (C-16). HRMS $\mathrm{C}_{25} \mathrm{H}_{27} \mathrm{BrN}_{2} \mathrm{O}_{2}[\mathrm{M}+\mathrm{H}]^{+}$calculated: 467.1329 , found 503.1328 . 
(3S,8aS)-7-(4'-bromo-[1,1'-biphenyl]-4-yl)-3-(tert-butyl)-2,8a-dimethyl-2,3,8,8atetrahydroimidazo[1,5-a]pyridine-1,5-dione 3e:
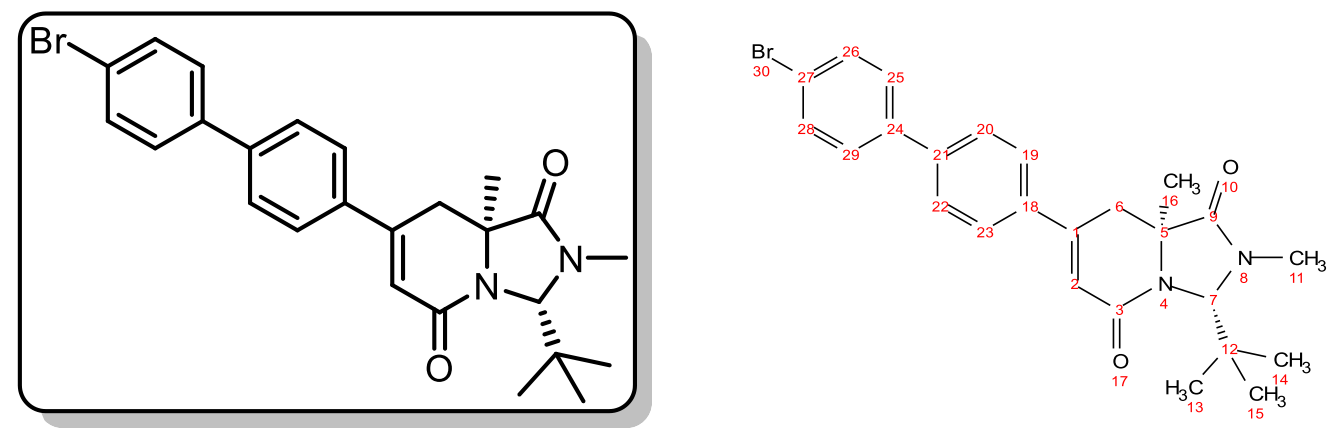

Following GP2, the title compound $3 \mathbf{e}$ was prepared from 2 e $(274 \mathrm{mg}, 0.54 \mathrm{mmol}, 1.0$ equiv.). The reaction was finished after $2 \mathrm{~h}$. Colourless oil (71 mg, 28\%). $\mathbf{R}_{f} 0.17\left(\mathrm{SiO}_{2}\right.$; pet. Ether/ EtOAc 4:1). $[\alpha]_{\mathrm{D}}^{26}=-168^{\circ}\left(c=1\right.$ in $\left.\mathrm{CHCl}_{3}\right)$.

IR (neat, cm ${ }^{-1}$ ): $v_{\max } 2982(\mathrm{C}-\mathrm{H}), 1705$ (C=O), 1676 (C=O), 1605. ${ }^{1} \mathbf{H}$ NMR (500 MHz, CDCl $\left.)_{3}\right) \delta .63-$ 7.55 (m, 6H, H-Ar), 7.47 (d, J = 8.5 Hz, 2H, H-Ar), 6.37 (d, J = 2.7 Hz, 1H, H-2), 5.27 (s, 1H, H-7), 3.07 (s, $3 \mathrm{H}, \mathrm{H}-11$ ), 3.02 (d, J = 16.6 Hz, 1H, H-6), 2.75 (dd, J = 16.6, $2.8 \mathrm{~Hz}, 1 \mathrm{H}, \mathrm{H}-6), 1.62(\mathrm{~s}, 3 \mathrm{H}, \mathrm{H}-16), 1.12$ (s, 9H, H-13, H-14, H-15). ${ }^{13} \mathrm{C}$ NMR (126 MHz, CDCl $)$ $\delta 173.8$ (C-3), 164.6 (C-9), 146.1 (C-Ar), 141.5 (CAr), 139.0 (C-Ar), 136.4 (C-1), 132.2 (CH-Ar), 128.7 (CH-Ar), 127.5 (CH-Ar), 126.6 (CH-Ar), 122.3 (CAr), 119.0 (CH-Ar), 81.2 (C-7), 60.7 (C-5), 38.0 (C-12), 36.9 (C-6), 31.5 (C-11), 27.6 (C-13, C-14, C-15), 22.8 (C-16). HRMS $\mathrm{C}_{25} \mathrm{H}_{27} \mathrm{BrN}_{2} \mathrm{O}_{2}[\mathrm{M}+\mathrm{H}]^{+}$calculated: 467.1329, found 467.1328.

\section{Isomerisation of $4 \mathrm{e}$ to $3 \mathrm{e}$}

Following GP4, 4e (70.0 mg, $0.23 \mathrm{mmol}, 1.0$ eq.) was dissolved in dry MeCN (0.90 mL, $0.25 \mathrm{M}$ ) before the addition of $\mathrm{DBU}\left(100 \mu \mathrm{L}, 0.68 \mathrm{mmol}, 3.0 \mathrm{eq}\right.$.). The reaction was heated to $150^{\circ} \mathrm{C}$ in a microwave reactor for $2 \mathrm{~h}$. The reaction was quenched with aqueous $\mathrm{HCl}(1.0 \mathrm{M})$. The reaction mixture was partitioned between $\mathrm{H}_{2} \mathrm{O}$ and EtOAc. The aqueous phase was further extracted with EtOAc $(x 2)$ and the combined organic phases were washed with brine, dried over $\mathrm{MgSO}_{4}$, filtered and the solvent was removed under reduced pressure. The crude product was purified by flash column chromatography $\left(\mathrm{SiO}_{2}\right.$, pet.ether/EtOAc, 4:6) to yield 3 e $(65.0 \mathrm{mg}, 93 \%)$ as a white solid. Data in agreement with those which are reported above. 
(3S,8aS)-3-(Tert-butyl)-2,8a-dimethyl-6-(trimethylsilyl)-2,3,8,8a-tetrahydroimidazo[1,5-a]pyridine1,5-dione 3k
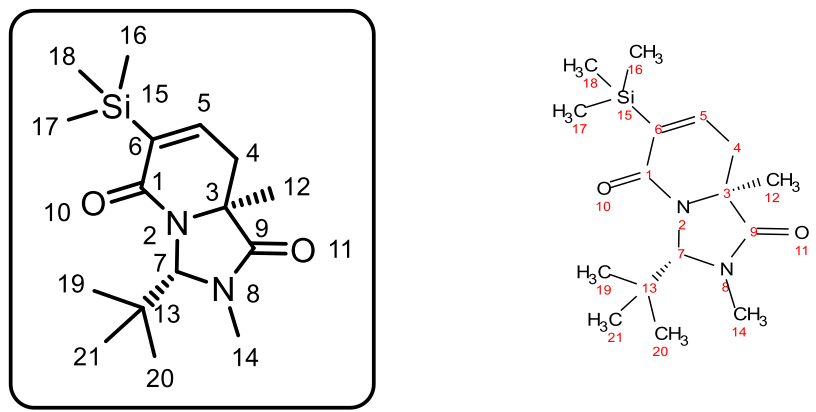

and (3S,7aS)-3-(Tert-butyl)-2,7a-dimethyl-6-((trimethylsilyl)methylene)tetrahydro-1H-pyrrolo[1,2c]imidazole-1,5(6H)-dione $7 \mathrm{k}$
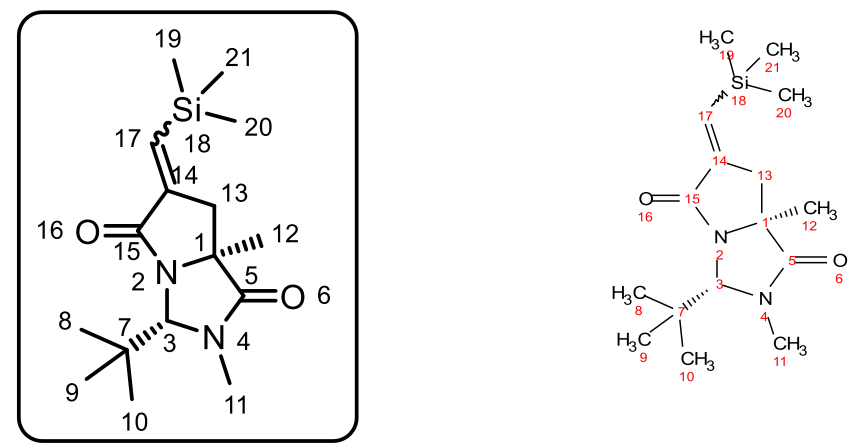

Following GP2, $N$-chloroformylimidazolidinone $\mathbf{2 k}(40.0 \mathrm{mg}, 0.116 \mathrm{mmol}, 1.0$ eq.) was dissolved in dry $\mathrm{MeCN}(0.58 \mathrm{~mL}, 0.2 \mathrm{M}$ ) before the addition of potassium iodide $(21.2 \mathrm{mg}, 0.128 \mathrm{mmol}, 1.1 \mathrm{eq}$.) and 2,6-lutidine ( $14.8 \mu \mathrm{L}, 0.128 \mathrm{mmol}, 1.1$ eq.). The reaction was heated to $150{ }^{\circ} \mathrm{C}$ in a microwave reactor for $2 \mathrm{~h}$. The reaction was quenched with aqueous $\mathrm{HCl}(1.0 \mathrm{M})$. The reaction mixture was partitioned between $\mathrm{H}_{2} \mathrm{O}$ and EtOAc. The aqueous phase was further extracted with EtOAc $(\times 2)$ and the combined organic phases were washed with brine, dried over $\mathrm{MgSO}_{4}$, filtered and the solvent was removed under reduced pressure. The residue was chromatographed $\left(\mathrm{SiO}_{2}\right.$, pet.ether/EtOAc, 4:6) to yield;

3a (7.8 mg, 22\%) as a white solid. Data in agreement with values reported above.

Mixed fractions were further purified by preparative TLC (pet.ether/EtOAC $=4: 6$ ) yielding;

3k $(2.5 \mathrm{mg}, 7 \%)$ as a white semi-solid. $\mathbf{R}_{f}=0.46$ (pet.ether/EtOAc $\left.=4: 6\right) ;[\alpha]^{24} \mathrm{D}=-80(c=0.5$ in $\mathrm{CHCl}_{3}$ ); IR (neat, $\mathrm{cm}^{-1}$ ): $v_{\max } 2958(\mathrm{C}-\mathrm{H}), 1703(\mathrm{C}=0) ;{ }^{1} \mathbf{H}$ NMR (400 MHz, $\left.\mathrm{CDCl}_{3}\right) \delta 6.69$ (dd, J=6.5, 2.3 $\mathrm{Hz}, 1 \mathrm{H}, \mathrm{H}-5), 5.27$ (s, 1H, H-7), 3.01 (d, J = 0.6 Hz, 3H, H-14), 2.49 (dd, J=17.0, $6.5 \mathrm{~Hz}, 1 \mathrm{H}, \mathrm{H}-4), 2.31$ (dd, $J=17.0,2.3 \mathrm{~Hz}, 1 \mathrm{H}, \mathrm{H}-4), 1.51$ (d, J = 0.6 Hz, 3H, H-12), 1.06 (s, 9H, H-19 + H-20 + H-21), 0.18 (s, 9H, H-16 + H-17 + H-18); $\left.{ }^{13} \mathrm{C} \mathrm{NMR} \mathrm{(101} \mathrm{MHz,} \mathrm{CDCl}\right) \delta 174.13$ (C-9), 166.52 (C-1), 143.58 (C-5), 139.45 (C-6), 80.72 (C-7), 60.79 (C-3), 37.62 (C-13), 35.14 (C-4), 31.27 (C-14), 27.52 (C-19 + C-20 + C-21), 22.75 (C-12), $-1.42(\mathrm{C}-16+\mathrm{C}-17+\mathrm{C}-18) ;$ HRMS C$_{16} \mathrm{H}_{28} \mathrm{~N}_{2} \mathrm{O}_{2} \mathrm{Si}[\mathrm{M}+\mathrm{Na}]^{+}$calculated: 331.1812 , found: 331.1817.

and; 
7k (mixture of $\mathrm{E}$ and $\mathrm{Z}$ isomers in a 2:1 ratio, data is reported for the major diastereomer) $3.4 \mathrm{mg}$, 9\%) as a white solid. $\mathbf{R}_{f}=0.31$ (pet.ether/EtOAc $\left.=4: 6\right) ;$;). $[\boldsymbol{\alpha}]_{\mathrm{D}}^{22}=-12\left(c=0.5\right.$ in $\left.\mathrm{CHCl}_{3}\right) ; \mathbf{m p} 148-150$

${ }^{\circ} \mathrm{C}$; IR (neat, $\mathrm{cm}^{-1}$ ): $v_{\max }$ 2958, 2926 (C-H), 1702 (C=O); ${ }^{1} \mathrm{H}$ NMR $\left(400 \mathrm{MHz}, \mathrm{CDCl}_{3}\right) \delta 6.70$ (d, J = $1.9 \mathrm{~Hz}$, $1 \mathrm{H}, \mathrm{H}-17), 4.79$ (s, 1H, H-3), 3.02 (d, J = 3.5 Hz, 1H, H-13), 2.97 (s, 3H, H-11), 2.84 (dd, J = 17.0, $1.9 \mathrm{~Hz}$, $1 \mathrm{H}, \mathrm{H}-13), 1.55$ (s, 3H, H-12), 1.09 (s, 9H, H-8 + H-9 + H-10), 0.16 (s, 9H, H-19+ H-20 + H-21); ${ }^{13} \mathrm{C} \mathrm{NMR}$ (101 MHz, CDCl $)$ ) 207.05 (C-5), 174.84 (C-15), 145.32 (C-14), 135.34 (C-17), 84.21 (C-3), 62.91 (C-1), 44.74 (C-13), 36.58 (C-7), 31.07 (C-11), 27.26 (C-8 + C-9 + C-10), 26.82 (C-12), -1.04 (C-19 + C-20 + C21); HRMS $\mathrm{C}_{16} \mathrm{H}_{28} \mathrm{~N}_{2} \mathrm{O}_{2} \mathrm{Si}[\mathrm{M}+\mathrm{H}]^{+}$calculated: 309.1993, found: 309.1991.

\section{Cyclisation conditions utilising $\mathrm{AlCl}_{3}$}

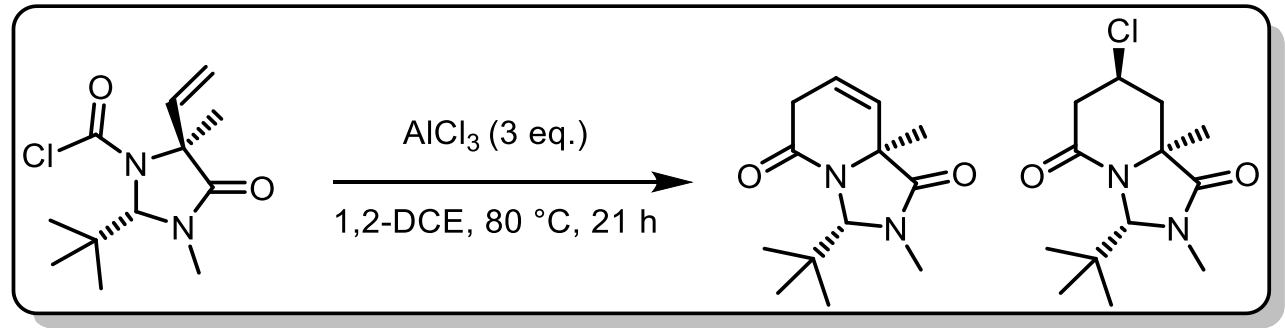

(3S,7S,8aS)-3-(Tert-butyl)-7-chloro-2,8a-dimethylhexahydroimidazo[1,5-a]pyridine-1,5-dione 5
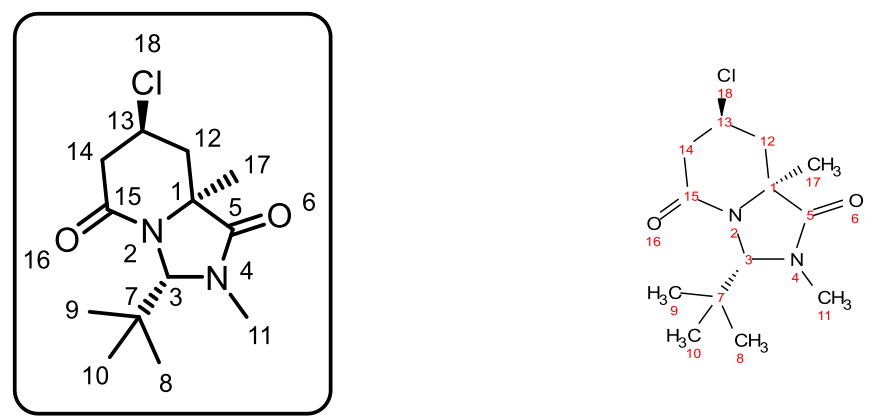

In a flame-dried microwave tube, $\mathrm{N}$-chloroformylimidazolidinone $1 \mathrm{1a}(36.0 \mathrm{mg}, 0.131 \mathrm{mmol}, 1.0 \mathrm{eq}$. was dissolved in 1,2-DCE (1.32 mL, $0.1 \mathrm{M})$ and aluminium chloride $(52.8 \mathrm{mg}, 0.395 \mathrm{mmol}, 3.0$ eq.) was added. The tube was sealed and heated in an oil bath at $80^{\circ} \mathrm{C}$ for $21 \mathrm{~h}$ (a dark brown/black mixture resulted after a few minutes). The reaction mixture was subsequently cooled to room temperature and quenched with aqueous $\mathrm{HCl}(1.0 \mathrm{M})$. The reaction mixture was extracted with $\mathrm{DCM}$ (x3) and combined organic layers were dried over $\mathrm{MgSO}_{4}$, filtered and the solvent was removed under reduced pressure. The residue was chromatographed $\left(\mathrm{SiO}_{2}\right.$, pet.ether/EtOAc, 4:6) to yield;

5 as a brown oil (16.5 mg, 46\% yield) ); $[\alpha]_{\mathrm{D}}^{25}=-16\left(c=0.5\right.$ in $\left.\mathrm{CHCl}_{3}\right)$; IR (neat, $\left.\mathbf{c m}^{-1}\right): v_{\max } 2978,2936$ (C-H), 1702, $1662(\mathrm{C}=\mathrm{O}) ;{ }^{1} \mathrm{H}$ NMR $\left(400 \mathrm{MHz}, \mathrm{CDCl}_{3}\right) \delta 5.22(\mathrm{~s}, 1 \mathrm{H}, \mathrm{H}-3), 4.48$ (dtd, J = 12.4, 7.4, $4.6 \mathrm{~Hz}$, $1 \mathrm{H}, \mathrm{H}-13$ ), 3.24 (dd, J=18.3, 8.2 Hz, 1H, H-14), 3.00 (s, 3H, H-11), 2.72 (dd, J = 18.3, 7.4 Hz, 1H, H-14), 2.61 (dd, $J=13.2,4.6 \mathrm{~Hz}, 1 \mathrm{H}, \mathrm{H}-12), 1.79(\mathrm{t}, J=12.4 \mathrm{~Hz}, 1 \mathrm{H}, \mathrm{H}-12), 1.55$ (s, 3H, H-17), 1.04 (s, 9H, H-8 + H-9 + H-10); ${ }^{13} \mathrm{C}$ NMR (101 MHz, CDCl $)$ ) 172.57 (C-5), 169.18 (C-15), 81.36 (C-3), 60.85 (C-1), 48.36 (C-13), 43.70 (C-12), 42.17 (C-14), 37.30 (C-7), 31.29 (C-11), 27.54 (C-8 + C-9 + C-10), 23.80 (C-17); HRMS $\mathrm{C}_{13} \mathrm{H}_{21} \mathrm{~N}_{2} \mathrm{O}_{2} \mathrm{Cl}[\mathrm{M}+\mathrm{H}]^{+}$calculated: 273.1364 , found: 273.1371 . The relative stereochemistry was confirmed in nOe experiments.

$4 a$ was isolated as a brown oil $(8.0 \mathrm{mg}, 22 \%$ yield). Data in agreement with values reported above. 


\section{Preparation of electrophiles}

\section{Synthesis of (3-Bromoprop-1-en-2-yl)arene}

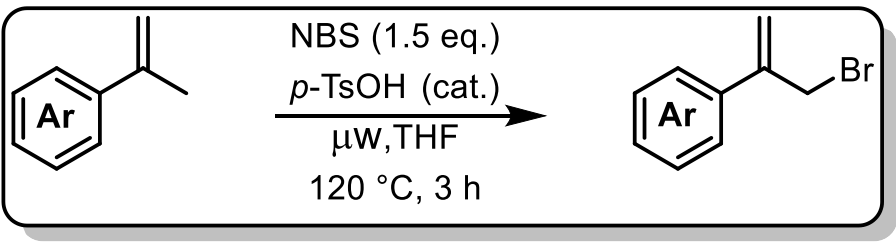

(3-Bromoprop-1-en-2-yl)benzene S3

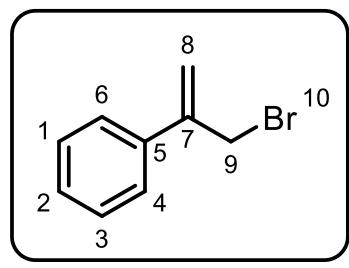
reduced pressure. The residue was purified by gravity column chromatography (hexane). Pure fractions were combined and concentrated under reduced pressure to yield the title compound as a yellow oil (1.06 gm, 30\% yield). ${ }^{1} \mathrm{H}$ NMR $\left(400 \mathrm{MHz}, \mathrm{CDCl}_{3}\right) \delta 7.57-7.31(\mathrm{~m}, 5 \mathrm{H}, \mathrm{H}-1+\mathrm{H}-2+\mathrm{H}-3+\mathrm{H}-4$ $+\mathrm{H}-6), 5.54(\mathrm{~d}, \mathrm{~J}=29.9 \mathrm{~Hz}, 2 \mathrm{H}, \mathrm{H}-8), 4.40(\mathrm{~s}, 2 \mathrm{H}, \mathrm{H}-9)$; Data in agreement with reported values. ${ }^{5}$

\section{1-(3-bromoprop-1-en-2-yl)-4-fluorobenzene S4}

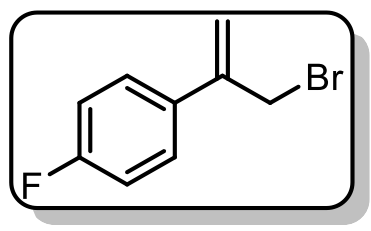

To a solution of 1-fluoro-4-(prop-1-en-2-yl)benzene $(1.00 \mathrm{~g}, 7.34 \mathrm{mmol}, 1.0$ eq.) in dry THF (15 ml, $0.5 \mathrm{M}$ ) were added $\mathrm{N}$-bromosuccinimide $(1.5 \mathrm{~g}, 8.81 \mathrm{mmol}, 1.2 \mathrm{eq}$.), $p$-TsOH $(0.28 \mathrm{~g}, 1.47 \mathrm{mmol}, 0.2$ eq.), and a colourless solution resulted. The mixture was heated to $120^{\circ} \mathrm{C}$ in the microwave for $3 \mathrm{~h}$ to give a brown solution. The reaction mixture was cooled to RT, then taken up in petroleum ether and washed with water $(3 \times 15 \mathrm{~mL})$. The combined organic phases were dried over $\mathrm{MgSO}_{4}$ and concentrated under reduced pressure. The residue was purified by gravity column chromatography (hexane). Pure fractions were combined and concentrated under reduced pressure to yield the title compound as a yellow oil ( $0.79 \mathrm{mg}, 50 \%$ yield). ${ }^{1} \mathrm{H} \mathrm{NMR}\left(500 \mathrm{MHz}, \mathrm{CDCl}_{3}\right) \delta 7.47$ (dd, J = 8.9, $5.3 \mathrm{~Hz}$, $2 \mathrm{H}, \mathrm{H}-3, \mathrm{H}-7), 7.11-6.97(\mathrm{~m}, 2 \mathrm{H}, \mathrm{H}-4, \mathrm{H}-6), 5.49$ (d, J = $13.0 \mathrm{~Hz}, 2 \mathrm{H}, \mathrm{H}-10), 4.36(\mathrm{~s}, 2 \mathrm{H}, \mathrm{H}-1) .{ }^{13} \mathrm{C}$ NMR (126 MHz, CDCl ${ }_{3}$ ) $\delta 163.8$ (C-5), 161.8 (C-5), 143.4 (C-9), 133.8 (C-2), 133.7 (C-2), 128.0 (C-3, C-7), 127.9 (C-3, C-7), 117.2 (C-10), 117.1 (C-10), 115.6 (C-4, C-6), 115.5 (C-4, C-6), 115.4 (C-4, C-6), 115.3 (C-4, C-6), $34.3(\mathrm{C}-1)$. 


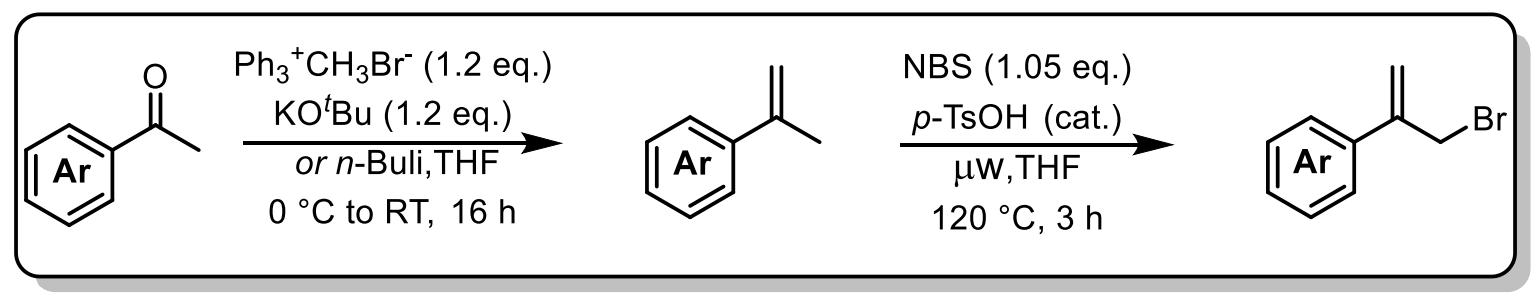

Step 1:

2-(Prop-1-en-2-yl)naphthalene S5

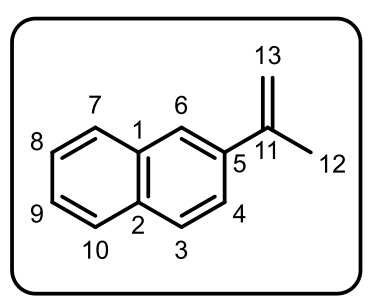

Methyltriphosphonium bromide (2.52 g, $7.05 \mathrm{mmol}, 1.2$ eq.) was dissolved in THF $(7.43 \mathrm{~mL}, 0.8 \mathrm{M})$ and cooled to $0{ }^{\circ} \mathrm{C}$. To the resulting white suspension was added $\mathrm{KO}^{\mathrm{t}} \mathrm{Bu}(1 \mathrm{M}$ in $\mathrm{THF}, 7.05 \mathrm{ml}, 1.2$ eq.) dropwise to give a yellow suspension which stirred at $0{ }^{\circ} \mathrm{C}$ for $45 \mathrm{~min}$. 1-(naphthalen-2yl)ethan-1-one ( $1.00 \mathrm{~g}, 5.88 \mathrm{mmol}, 1$ eq.) in THF $(8.4 \mathrm{~mL}, 0.8 \mathrm{M})$ was added dropwise. An orange mixture resulted and after stirring overnight (16 h) this became red. The mixture was filtered by gravity filtration and washed with petroleum ether ( $3 x$ $15 \mathrm{~mL}$ ). The mother liquor was concentrated under reduced pressure. The residue was chromatographed ( $\mathrm{SiO}_{2}$, pet.ether) to yield the title compound as a white solid $(758 \mathrm{mg}, 77 \%$ yield). IR (neat, $\left.\mathrm{cm}^{-1}\right): v_{\max } 2988,2972,2901 ; \mathrm{mp} 58-60{ }^{\circ} \mathrm{C} ;{ }^{1} \mathrm{H}$ NMR $\left(400 \mathrm{MHz}, \mathrm{CDCl}_{3}\right) \delta 7.89-7.76(\mathrm{~m}, 4 \mathrm{H}$, $\mathrm{H}-3+\mathrm{H}-6+\mathrm{H}-7+\mathrm{H}-10), 7.68$ (dd, $J=8.6,1.9 \mathrm{~Hz}, 1 \mathrm{H}, \mathrm{H}-4$ ), 7.46 (ddd, $J=6.6,3.8,1.9 \mathrm{~Hz}, 2 \mathrm{H}, \mathrm{H}-8+\mathrm{H}-$ 9), $5.54(\mathrm{~s}, 1 \mathrm{H}, \mathrm{H}-13), 5.20(\mathrm{~s}, 1 \mathrm{H}, \mathrm{H}-13), 2.28(\mathrm{~s}, 3 \mathrm{H}, \mathrm{H}-12) ;{ }^{13} \mathrm{C} \mathrm{NMR}\left(101 \mathrm{MHz}, \mathrm{CDCl}_{3}\right) \delta 143.1$ (C-5), 138.5 (C-1), 133.5 (C-11), 132.9 (C-2), 128.4 (C-7 or C-10), 127.8 (C-7 or C-10), 127.6 (C-3), 126.3 (C-8 or C-9), 126.0 (C-8 or C-9), 124.4 (C-6), 124.0 (C-4), 113.2 (C-13), 22.1 (C-12); HRMS $\mathrm{C}_{13} \mathrm{H}_{12}[\mathrm{M}+\mathrm{H}]^{+}$ calculated: 169.1012 , found: 169.1008 . Data in agreement with reported values. ${ }^{6}$

\section{2-(3-Bromoprop-1-en-2-yl)naphthalene S6}

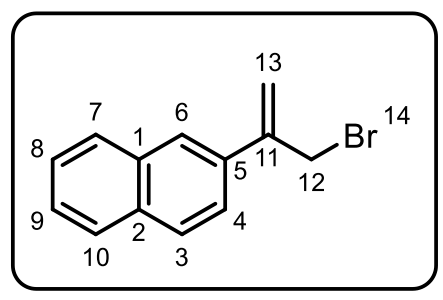

$\mathrm{N}$-bromosuccinimide ( $423 \mathrm{mg}, 2.38 \mathrm{mmol}, 2.0$ eq.) and $p$ - $\mathrm{TsOH}(45.2$ $\mathrm{mg}, 0.24 \mathrm{mmol}, 0.2$ eq.) were dissolved in dry THF $(3.97 \mathrm{~mL})$ to give a yellow solution. 2-(prop-1-en-2-yl)naphthalene S5 (200 mg, 1.19 $\mathrm{mmol}, 1.0$ eq.) was added and a colourless solution resulted. The mixture was heated to $130{ }^{\circ} \mathrm{C}$ in the microwave for $3 \mathrm{~h}$ to give a brown solution. The reaction mixture was poured into pet. Ether (a solid immediately precipitated). The organic layer was washed with water $(4 \times 10 \mathrm{~mL})$ and brine (once) becoming a yellow solution. The organic layer was dried over $\mathrm{MgSO}_{4}$, filtered and concentrated under reduced pressure. The residue was chromatographed $\left(\mathrm{SiO}_{2}\right.$, pet.ether) to yield the title compound as colourless oil (104 mg, 35\% yield). IR (neat, $\mathbf{c m}^{-1}$ ): $\mathbf{V}_{\max } 2988,2972,2901 ;{ }^{1} \mathbf{H}$ NMR (400 MHz, CDCl $\left.{ }_{3}\right) \delta 7.96-7.76(\mathrm{~m}, 4 \mathrm{H}, \mathrm{H}-3+\mathrm{H}-6+\mathrm{H}-7+\mathrm{H}-10), 7.61$ (dd, J = 8.6, $1.9 \mathrm{~Hz}, 1 \mathrm{H}, \mathrm{H}-$ 4), $7.48(\mathrm{td}, J=6.3,5.9,3.4 \mathrm{~Hz}, 2 \mathrm{H}, \mathrm{H}-8+\mathrm{H}-9), 5.70(\mathrm{~s}, 1 \mathrm{H}, \mathrm{H}-13), 5.58(\mathrm{~s}, 1 \mathrm{H}, \mathrm{H}-13), 4.49(\mathrm{~s}, 2 \mathrm{H}, \mathrm{H}-$ 12). Data in agreement with reported values. ${ }^{7}$ 


\section{1-(but-1-en-2-yl)-3-methoxybenzene S7}

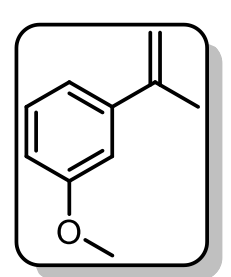<smiles>C=C(C)c1cccc(OC)c1</smiles>

Methyltriphosphonium bromide $(14.00 \mathrm{~g}, 39.60 \mathrm{mmol}, 1.2 \mathrm{eq}$.) was dissolved in THF (60 mL, $0.8 \mathrm{M})$ and cooled to $0{ }^{\circ} \mathrm{C}$. To the resulting white suspension was added $\mathrm{KO}^{t} \mathrm{Bu}(4.44 \mathrm{gm}, 1.2$ eq.) dropwise to give a yellow suspension which stirred at $0{ }^{\circ} \mathrm{C}$ for $45 \mathrm{~min}$. 1-(3-methoxyphenyl)ethan-1-one (5.00 g, $33.00 \mathrm{mmol}, 1$ eq.) in THF $(8.4 \mathrm{~mL}, 0.8 \mathrm{M})$ was added dropwise. An orange mixture resulted and after stirring overnight $(16 \mathrm{~h})$ this became red. The mixture was filtered by gravity filtration and washed with petroleum ether $(3 \times 50 \mathrm{~mL})$. The mother liquor was concentrated under reduced pressure. The residue was chromatographed $\left(\mathrm{SiO}_{2}\right.$, pet.ether) to yield the title compound as a white solid ( $3.00 \mathrm{gm}, 61 \%$ yield).

${ }^{1} \mathrm{H}$ NMR (500 MHz, CDCl $) \delta 7.29$ (t, J=8.0 Hz, 1H, H-4), 7.11 (ddd, J = 7.7, 1.7, $\left.0.9 \mathrm{~Hz}, 1 \mathrm{H}, \mathrm{H}-7\right), 7.05$ (dd, $J=2.6,1.7 \mathrm{~Hz}, 1 \mathrm{H}, \mathrm{H}-5$ ), 6.86 (ddd, $J=8.1,2.6,1.0 \mathrm{~Hz}, 1 \mathrm{H}, \mathrm{H}-3$ ), 5.41 (dd, $J=1.6,0.8 \mathrm{~Hz}, 1 \mathrm{H}, \mathrm{H}-9$ ), $5.13(\mathrm{t}, J=1.6,0.8 \mathrm{~Hz}, 1 \mathrm{H}, \mathrm{H}-9), 3.85(\mathrm{~s}, 3 \mathrm{H}, \mathrm{H}-11), 2.22-2.14(\mathrm{~m}, 3 \mathrm{H}, \mathrm{H}-12) .{ }^{13} \mathrm{C} \mathrm{NMR}(126 \mathrm{MHz}$, $\mathrm{CDCl}_{3}$ ) $\delta 159.6$ (C-6), 143.3 (C-8), 142.9 (C-2), 129.3 (CH-Ar), 118.2 (CH-Ar), 112.8 (CH-Ar), 112.7 (CHAr), 111.6 (C-9), 55.3 (C-11), 22.0 (C-1, C-12).

\section{1-(3-bromoprop-1-en-2-yl)-3-methoxybenzene S8}

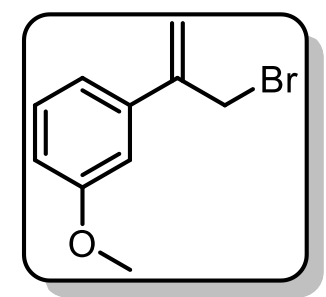<smiles></smiles>

To a solution of 1-methoxy-3-(prop-1-en-2-yl)benzene $\mathbf{S 7}$ (3.00 g, $20.24 \mathrm{mmol}, 1.0 \mathrm{eq}$.) in dry THF (15 $\mathrm{ml}, 1.5 \mathrm{M}$ ) were added $\mathrm{N}$-bromosuccinimide $(3.48 \mathrm{~g}, 19.84 \mathrm{mmol}, 0.98 \mathrm{eq}$.), $p$ - $\mathrm{TsOH}(0.77 \mathrm{~g}, 4.05$ mmol, 0.2 eq.), and added a colourless solution resulted. The mixture was heated to $120{ }^{\circ} \mathrm{C}$ in the microwave for $3 \mathrm{~h}$ to give a brown solution. The reaction mixture was cooled to $\mathrm{RT}$, then taken up in petroleum ether and washed with water $(3 \times 15 \mathrm{~mL})$. The combined organic phases were dried over $\mathrm{MgSO}_{4}$ and concentrated under reduced pressure. The residue was purified by gravity column chromatography (hexane). Pure fractions were combined and concentrated under reduced pressure to yield the title compound as a yellow oil $(2.5,54 \%$ yield).

${ }^{1} \mathrm{H}$ NMR $\left(500 \mathrm{MHz}, \mathrm{CDCl}_{3}\right) \delta 7.31(\mathrm{t}, J=8.0 \mathrm{~Hz}, 1 \mathrm{H}, \mathrm{H}-4), 7.09$ (ddd, J = 7.7, 1.7, $\left.0.9 \mathrm{~Hz}, 1 \mathrm{H}, \mathrm{H}-7\right), 7.05$ (dd, $J=2.6,1.7 \mathrm{~Hz}, 1 \mathrm{H}, \mathrm{H}-5$ ), 6.90 (ddd, $J=8.2,2.6,0.9 \mathrm{~Hz}, 1 \mathrm{H}, \mathrm{H}-3$ ), 5.57 (s, $1 \mathrm{H}, \mathrm{H}-9), 5.50$ (s, $1 \mathrm{H}, \mathrm{H}-$ 9), 4.38 (s, 2H, H-1), 3.84 (s, 3H, H-11). ${ }^{13} \mathrm{C} \mathrm{NMR} \mathrm{(126} \mathrm{MHz,} \mathrm{CDCl} 3$ ) $\delta 159.8$ (C-6), 144.3 (C-8), 139.2 (C2), 129.6 (CH-Ar), 118.7 (CH-Ar), 117.6 (CH-Ar), 113.6 (CH-Ar), 112.3 (CH-Ar), 55.4 (C-11), 34.3 (C-1). 


\section{1,2-dichloro-4-(prop-1-en-2-yl)benzene S9}

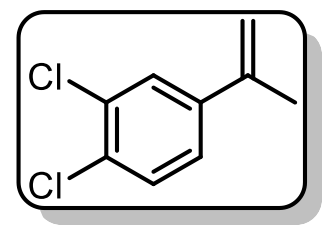<smiles></smiles>

Methyltriphosphonium bromide (10.00 g, $28.60 \mathrm{mmol}, 1.1$ eq.) was dissolved in THF (45 mL, $1.0 \mathrm{M})$ and cooled to $0{ }^{\circ} \mathrm{C}$. To the resulting white suspension was added $n$-BuLi (11.44 ml, $2.5 \mathrm{M}$ in THF, 1.1 eq.) dropwise to give a yellow suspension which stirred at $0{ }^{\circ} \mathrm{C}$ for $45 \mathrm{~min}$. 1-(3,4dichlorophenyl)ethan-1-one (5.00 g, $26.00 \mathrm{mmol}, 1$ eq.) in THF (20 mL, $1.3 \mathrm{M})$ was added dropwise. An orange mixture resulted and after stirring overnight $(16 \mathrm{~h})$ this became red. The mixture was filtered by gravity filtration and washed with petroleum ether $(3 \times 50 \mathrm{~mL})$. The mother liquor was concentrated under reduced pressure. The residue was chromatographed $\left(\mathrm{SiO}_{2}\right.$, pet.ether) to yield the title compound as a white solid ( $3.40 \mathrm{gm}, 70 \%$ yield).

${ }^{1} \mathrm{H}$ NMR $\left(500 \mathrm{MHz}, \mathrm{CDCl}_{3}\right) \delta 7.54(\mathrm{~d}, J=2.2 \mathrm{~Hz}, 1 \mathrm{H}, \mathrm{H}-3), 7.39$ (d, $\left.J=8.4 \mathrm{~Hz}, 1 \mathrm{H}, \mathrm{H}-6\right), 7.29$ (dd, $J=8.4$, $2.1 \mathrm{~Hz}, 1 \mathrm{H}, \mathrm{H}-7), 5.43-5.34(\mathrm{~m}, 1 \mathrm{H}, \mathrm{H}-10), 5.22-5.13(\mathrm{~m}, 1 \mathrm{H}, \mathrm{H}-10), 2.13(\mathrm{~s}, 3 \mathrm{H}, \mathrm{H}-1) .{ }^{13} \mathrm{C}$ NMR (126 $\mathrm{MHz}, \mathrm{CDCl}_{3}$ ) $\delta 141.3$ (C-9), 141.2 (C-2), 132.4 (C-4), 131.3 (C-5), 130.8 (C-6), 127.6 (C-3), 124.9 (C-7), 114.1 (C-10), 21.6 (C-1).

\section{4-(3-bromoprop-1-en-2-yl)-1,2-dichlorobenzene S10}

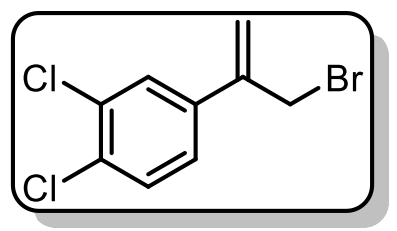<smiles>C=C(CBr)c1ccc(Cl)c(Cl)c1</smiles>

To a solution of 1,2-dichloro-4-(prop-1-en-2-yl)benzene $\mathbf{S 9}(2.18 \mathrm{~g}, 11.69 \mathrm{mmol}, 1.0 \mathrm{eq}$.) in dry THF $(8.00 \mathrm{ml}, 1.5 \mathrm{M})$ were added $\mathrm{N}$-bromosuccinimide $(2.00 \mathrm{~g}, 11.45 \mathrm{mmol}, 0.98 \mathrm{eq}$.), $p$-TsOH $(0.44 \mathrm{~g}$, $2.33 \mathrm{mmol}, 0.2$ eq.), and a colourless solution resulted. The mixture was heated to $120{ }^{\circ} \mathrm{C}$ in the microwave for $3 \mathrm{~h}$ to give a brown solution. The reaction mixture was cooled to $\mathrm{RT}$, then taken up in petroleum ether and washed with water $(3 \times 25 \mathrm{~mL}$ ). The combined organic phases were dried over $\mathrm{MgSO}_{4}$ and concentrated under reduced pressure. The residue was purified by gravity column chromatography (hexane). Pure fractions were combined and concentrated under reduced pressure to yield the title compound as a white solid (2.3,73\% yield).

${ }^{1} \mathrm{H}$ NMR $\left(500 \mathrm{MHz}, \mathrm{CDCl}_{3}\right) \delta 7.59$ (d, $\left.J=2.2 \mathrm{~Hz}, 1 \mathrm{H}, \mathrm{H}-3\right), 7.46$ (d, $\left.J=8.4 \mathrm{~Hz}, 1 \mathrm{H}, \mathrm{H}-6\right), 7.34$ (dd, $J=8.4$, $2.2 \mathrm{~Hz}, 1 \mathrm{H}, \mathrm{H}-7), 5.57$ (d, J = 7.4 Hz, 2H, H-10), 4.34 (s, 2H, H-1). ${ }^{13} \mathrm{C}$ NMR $\left(126 \mathrm{MHz}, \mathrm{CDCl}_{3}\right) \delta 142.4$ (C9), 137.8 (C-2), 132.8 (C-4), 132.4 (C-5), 130.5 (C-6), 128.3 (C-3), 125.6 (C-7), 118.7 (C-10), 33.5 (C-1). 


\section{4-bromo-4'-(prop-1-en-2-yl)-1,1'-biphenyl S11}
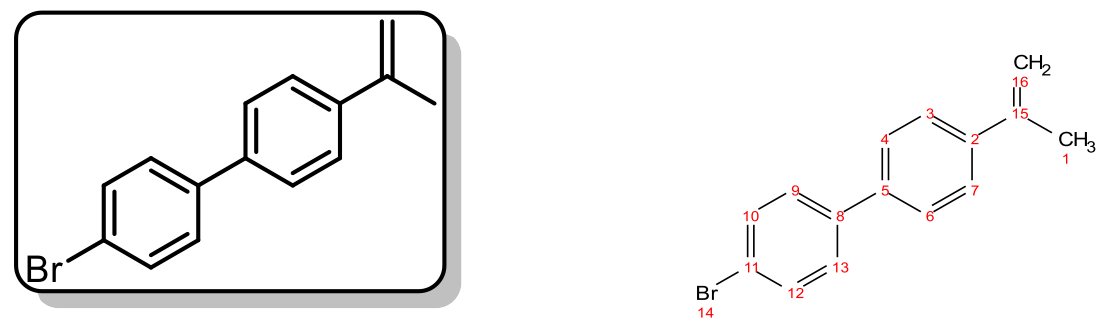

Methyltriphosphonium bromide ( $4.29 \mathrm{~g}, 11.99 \mathrm{mmol}, 1.1$ eq.) was dissolved in THF (22 mL, $0.5 \mathrm{M})$ and cooled to $0{ }^{\circ} \mathrm{C}$. To the resulting white suspension was added $n$-BuLi $(4.80 \mathrm{ml}, 2.5 \mathrm{M}$ in THF, 1.1 eq.) dropwise to give a yellow suspension which stirred at $0{ }^{\circ} \mathrm{C}$ for $45 \mathrm{~min}$. 1-(4'-bromo-[1,1'biphenyl]-4-yl)ethan-1-one ( $3.00 \mathrm{~g}, 11.00 \mathrm{mmol}, 1 \mathrm{eq}$.) in THF (11 mL, $1.0 \mathrm{M})$ was added dropwise. An orange mixture resulted and after stirring overnight $(16 \mathrm{~h})$ this became red. The mixture was filtered by gravity filtration and washed with petroleum ether $(3 \times 50 \mathrm{~mL})$. The mother liquor was concentrated under reduced pressure. The residue was chromatographed $\left(\mathrm{SiO}_{2}\right.$, pet.ether) to yield the title compound as a white solid ( $1.94 \mathrm{gm}, 65 \%$ yield).

${ }^{1} \mathrm{H}$ NMR $\left(500 \mathrm{MHz}, \mathrm{CDCl}_{3}\right) \delta 7.59-7.51(\mathrm{~m}, 6 \mathrm{H}, \mathrm{H}-\mathrm{Ar}), 7.49-7.44(\mathrm{~m}, 2 \mathrm{H}, \mathrm{H}-\mathrm{Ar}), 5.57-5.35(\mathrm{~m}, 1 \mathrm{H}$, $\mathrm{H}-16), 5.24-4.98(\mathrm{~m}, 1 \mathrm{H}, \mathrm{H}-16), 2.19(\mathrm{~s}, 3 \mathrm{H}, \mathrm{H}-1) .{ }^{13} \mathrm{C}$ NMR $\left(126 \mathrm{MHz}, \mathrm{CDCl}_{3}\right) \delta 142.8$ (C-15), 140.7 (C-2), 139.8 (CH-Ar), 139.1 (CH-Ar), 132.0 (CH-Ar), 128.7 (CH-Ar), 126.9 (CH-Ar), 126.2 (CH-Ar), 121.7 (C-Ar), 112.9 (C-16), 21.9 (C-1). 


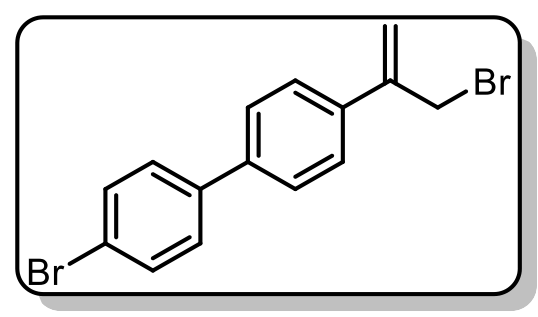<smiles></smiles>

To a solution of 4-bromo-4'-(prop-1-en-2-yl)-1,1'-biphenyl S11 (1.22 g, $4.46 \mathrm{mmol}, 1.0$ eq.) in dry THF $(3.00 \mathrm{ml}, 1.5 \mathrm{M})$ were added $\mathrm{N}$-bromosuccinimide $(0.81 \mathrm{~g}, 4.40 \mathrm{mmol}, 0.98 \mathrm{eq}$.), $p$ - $\mathrm{TsOH}(0.17 \mathrm{~g}, 0.89$ mmol, 0.2 eq.), and a colourless solution resulted. The mixture was heated to $120{ }^{\circ} \mathrm{C}$ in the microwave for $3 \mathrm{~h}$ to give a brown solution. The reaction mixture was cooled to $\mathrm{RT}$, then taken up in petroleum ether and washed with water $(3 \times 15 \mathrm{~mL})$. The combined organic phases were dried over $\mathrm{MgSO}_{4}$ and concentrated under reduced pressure. The residue was purified by gravity column chromatography (hexane). Pure fractions were combined and concentrated under reduced pressure to yield the title compound as a white solid $(0.99,62 \%$ yield).

${ }^{1} \mathrm{H}$ NMR $\left(500 \mathrm{MHz}, \mathrm{CDCl}_{3}\right) \delta 7.61-7.55$ (m, 6H, H-Ar), $7.50-7.44(\mathrm{~m}, 2 \mathrm{H}, \mathrm{H}-\mathrm{Ar}), 5.59$ (d, J = $48.5 \mathrm{~Hz}$, 2H, H-16), 4.43 (s, 2H, H-1). ${ }^{13} \mathrm{C}$ NMR (126 MHz, CDCl $) \delta 143.7$ (C-15), 139.9 (C-Ar), 139.4 (C-Ar), 136.8 (C-Ar), 132.0 (CH-Ar), 128.7 (CH-Ar), 127.1 (CH-Ar), 126.7 (CH-Ar), 121.9 (C-Ar), 117.4 (C-16), $34.1(\mathrm{C}-1)$.

\section{Synthesis of (E)-(3-Bromoprop-1-en-1-yl)trimethylsilane S14}

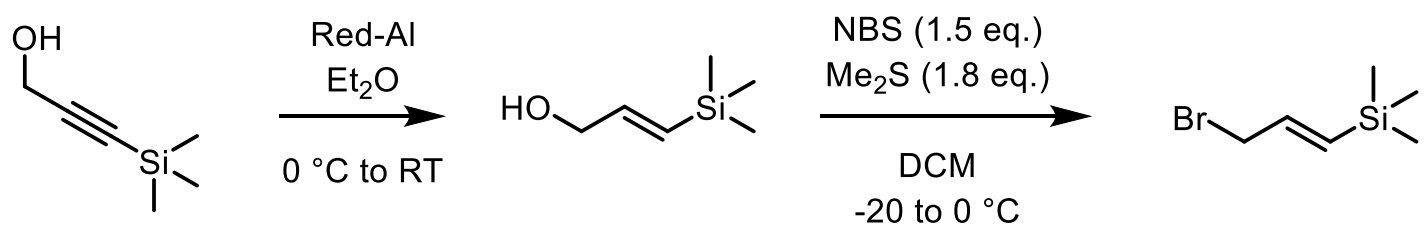

\section{Step 1:}

(E)-3-(Trimethylsilyl)prop-2-en-1-ol S13

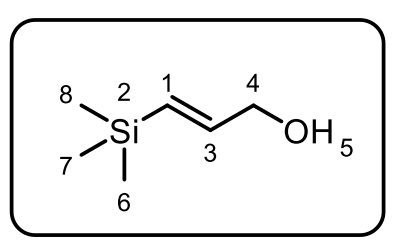

3-(Trimethylsilyl)prop-2-yn-1-ol $(1.16 \mathrm{~mL}, 7.80 \mathrm{mmol}, 1.0$ eq.) was dissolved in dry $\mathrm{Et}_{2} \mathrm{O}(16.0 \mathrm{~mL})$ and cooled to $0{ }^{\circ} \mathrm{C}$ in an ice-bath. Red-Al ${ }^{\circ}$ (3.55 ml, $10.92 \mathrm{mmol}, 1.4$ eq.) was added dropwise. The ice-bath was removed and the reaction stirred at RT for $2 \mathrm{~h}$. The reaction was cooled to $0{ }^{\circ} \mathrm{C}$ and quenched by the addition of water $(2 \mathrm{~mL})$ resulting in a white precipitate. $\mathrm{H}_{2} \mathrm{SO}_{4}$ (aq.) $(3.6 \mathrm{M}, 20 \mathrm{~mL})$ was added. The reaction mixture was extracted with $\mathrm{Et}_{2} \mathrm{O}(3 \mathrm{x}$ $50 \mathrm{~mL})$. The combined organic layers were washed with water $(20 \mathrm{~mL})$ and then brine $(20 \mathrm{~mL})$, dried over $\mathrm{MgSO}_{4}$, filtered and concentrated under reduced pressure. The title compound was yielded as a colourless oil ( $820 \mathrm{mg}, 80 \%$ yield) and used without further purification. ${ }^{1} \mathbf{H}$ NMR ( $\left.400 \mathrm{MHz}, \mathrm{CDCl}_{3}\right) \delta$ $6.17(\mathrm{dt}, J=18.8,4.4 \mathrm{~Hz}, 1 \mathrm{H}, \mathrm{H}-3), 5.90(\mathrm{dt}, J=18.8,1.7 \mathrm{~Hz}, 1 \mathrm{H}, \mathrm{H}-1), 4.16$ (dd, $J=4.4,1.7 \mathrm{~Hz}, 2 \mathrm{H}, \mathrm{H}-$ 4), $1.79(\mathrm{~s}, 1 \mathrm{H}, \mathrm{H}-5), 0.07(\mathrm{~s}, 9 \mathrm{H}, \mathrm{H}-7+\mathrm{H}-8+\mathrm{H}-9)$. Data in agreement with reported values. ${ }^{3}$ 


\section{Step 2:}

\section{(E)-(3-Bromoprop-1-en-1-yl)trimethylsilane S14}

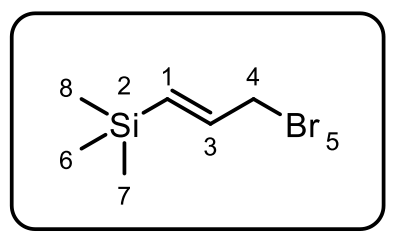

$\mathrm{N}$-bromosuccinimide (614 $\mathrm{mg}, 3.45 \mathrm{mmol}, 1.5$ eq.) was dissolved in DCM $(6.0 \mathrm{~mL}, 0.3 \mathrm{M})$ and cooled to $0{ }^{\circ} \mathrm{C}$. Dimethyl sulfide $(257.6 \mathrm{mg}, 4.15$ $\mathrm{mmol}, 1.8 \mathrm{eq}$.) was added dropwise over a period of $3 \mathrm{~min}$. resulting in a yellow precipitate. The mixture was cooled to $-20{ }^{\circ} \mathrm{C}$ and $(E)-3-$ (trimethylsilyl)prop-2-en-1-ol 89 (300 mg, $2.30 \mathrm{mmol}, 1.0$ eq.) in DCM

$\left(2.4 \mathrm{~mL}\right.$ ) was added dropwise over a period of $3 \mathrm{~min}$. The reaction was warmed to $0{ }^{\circ} \mathrm{C}$ and stirred for $3 \mathrm{~h}$. The reaction mixture was diluted with pentane then poured into ice water. The organic phase was washed with brine $(10 \mathrm{~mL})$, filtered through silica gel $(500 \mathrm{mg})$ and concentrated under reduced pressure to yield the title compound ( $323 \mathrm{mg}, 73 \%$ yield) as a colourless liquid. IR (neat, $\mathbf{~ c m}^{-}$ $\left.{ }^{1}\right): v_{\max } 3410,2925 ;{ }^{1} \mathrm{H}$ NMR $\left(400 \mathrm{MHz}, \mathrm{CDCl}_{3}\right) \delta 6.14$ (dt, J=18.2, $6.8 \mathrm{~Hz}, 1 \mathrm{H}, \mathrm{H}-3$ ), 5.95 (dt, J = 18.2, $1.0 \mathrm{~Hz}, 1 \mathrm{H}, \mathrm{H}-1), 3.96(\mathrm{~d}, J=6.8 \mathrm{~Hz}, 1 \mathrm{H}, \mathrm{H}-4), 0.08(\mathrm{~s}, 8 \mathrm{H}, \mathrm{H}-6+\mathrm{H}-7+\mathrm{H}-8) .{ }^{13} \mathrm{C}$ NMR $\left(126 \mathrm{MHz}, \mathrm{CDCl}_{3}\right)$ $\delta 140.8(\mathrm{C}-3), 135.9$ (C-1), 35.4 (C-4), -1.4 (C-6 + C-7 + C-8). Data in agreement with reported values. ${ }^{4}$

\section{Functionalization of the dihydropyridinone scaffold}

\section{Dihydroxylation derivatisations}

(3S,6S,7S,8aS)-3-(tert-butyl)-6,7-dihydroxy-2,8a-dimethylhexahydroimidazo[1,5-a]pyridine-1,5dione 10a
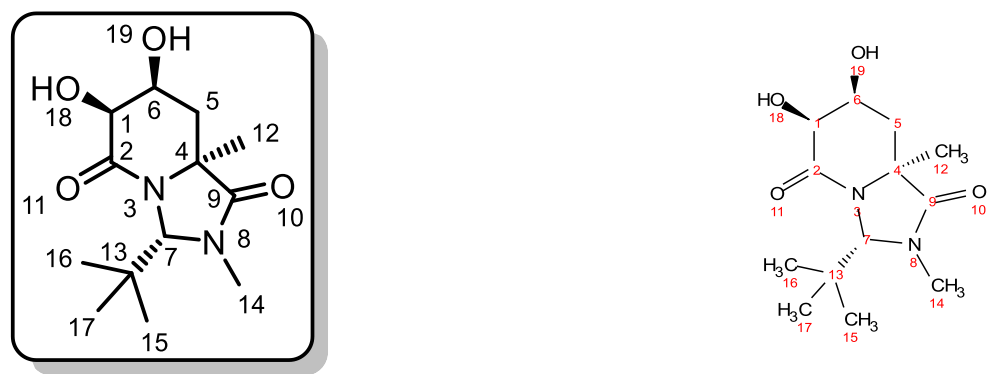

NMO (88.8 mg, $0.757 \mathrm{mmol}, 2.0$ eq.) and osmium tetroxide ( $48.3 \mu \mathrm{L}, 0.008 \mathrm{mmol}, 0.02$ eq.) were added to (3S,8aS)-3-(Tert-butyl)-2,8a-dimethyl-2,3,8,8a-tetrahydroimidazo[1,5-a]pyridine-1,5-dione $3 a$ ( $89.5 \mathrm{mg}, 0.379 \mathrm{mmol}, 1.0$ eq.) in dry $\mathrm{MeCN}(1.50 \mathrm{~mL})$. The reaction stirred for $24 \mathrm{~h}$ at RT. The reaction was quenched with sat. $\mathrm{Na}_{2} \mathrm{~S}_{2} \mathrm{O}_{3}$ (aq.). The reaction mixture was extracted with EtOAc (3x). The combined organic phases were washed with brine, dried over $\mathrm{MgSO}_{4}$ and concentrated under reduced pressure. The residue was chromatographed $\left(\mathrm{SiO}_{2}\right.$, pet ether/ EtOAc 1:9) to yield the title compound as an off-white semi-solid (56.2 mg, 49\% yield). $\mathbf{R}_{f}=0.09$ (EtOAc). [ $\left.\boldsymbol{\alpha}\right]_{\mathrm{D}}^{25}=-4$ (c=1 in $\mathrm{CHCl}_{3}$ ); IR (neat, cm ${ }^{-1}$ ): $v_{\max }$ 3675, 3399 (br) (OH), 2970, 2922 (C-H), 1683 (C=O); ${ }^{1} \mathrm{H}$ NMR (500 MHz, $\left.\mathrm{CDCl}_{3}\right) \delta 5.07(\mathrm{~s}, 1 \mathrm{H}, \mathrm{H}-7), 4.42-4.35(\mathrm{~m}, 1 \mathrm{H}, \mathrm{H}-1), 4.02-3.97(\mathrm{~m}, 1 \mathrm{H}, \mathrm{H}-6), 3.93(\mathrm{~d}, J=1.7 \mathrm{~Hz}, 1 \mathrm{H}, \mathrm{H}-$ 18), 3.04 (t, $J=1.7 \mathrm{~Hz}, 1 \mathrm{H}, \mathrm{H}-19$ ), 2.98 (s, 3H, H-14), 2.50 (dd, J = 14.7, 4.4 Hz, 1H, H-5), 1.79 (s, 3H, H12), $1.70(\mathrm{~s}, 1 \mathrm{H}, \mathrm{H}-5), 1.12$ (s, 9H, H-15 + H-16 + H-17); ${ }^{13} \mathrm{C} \mathrm{NMR}\left(126 \mathrm{MHz}, \mathrm{CDCl}_{3}\right) \delta 174.1$ (C-9), 171.8 (C-2), 82.6 (C-7), 70.8 (C-6), 65.8 (C-1), 60.8 (C-4), 36.5 (C-13), 35.7 (C-5), 30.7 (C-14), 28.3 (C-15 + C$16+\mathrm{C}-17), 27.4(\mathrm{C}-12)$. HRMS $\mathrm{C}_{13} \mathrm{H}_{22} \mathrm{~N}_{2} \mathrm{O}_{4}[\mathrm{M}+\mathrm{H}]^{+}$calculated: 271.1652 , found: 271.1651. 
(3S,6S,7S,8aS)-3-(tert-butyl)-6,7-dihydroxy-2,6,8a-trimethylhexahydroimidazo[1,5-a]pyridine-1,5dione 10c
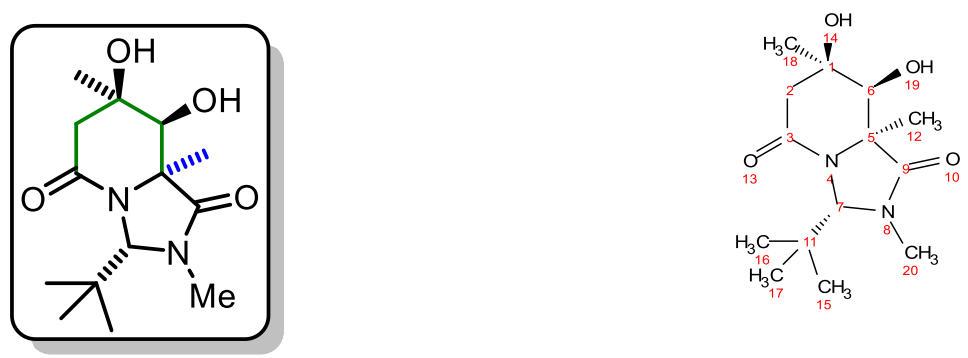

NMO (16.80 mg, $0.14 \mathrm{mmol}, 2.0$ eq.) and osmium tetroxide $(46.0 \mu \mathrm{L}, 0.008 \mathrm{mmol}, 0.02$ eq.) were added to $4 \mathrm{~h}$ (18.00 $\mathrm{mg}, 0.72 \mathrm{mmol}, 1.0$ eq.) in dry MeCN (1.50 mL). The reaction stirred for $24 \mathrm{~h}$ at RT. The reaction was quenched with sat. $\mathrm{Na}_{2} \mathrm{~S}_{2} \mathrm{O}_{3}$ (aq.). The reaction mixture was extracted with EtOAc $(3 \times 1)$. The combined organic phases were washed with brine, dried over $\mathrm{MgSO}_{4}$ and concentrated under reduced pressure. The residue was chromatographed $\left(\mathrm{SiO}_{2}\right.$, pet ether/ EtOAc 1:9) to yield the title compound as an off-white semi-solid (4.90 mg, 51\% yield). $\mathbf{R}_{f}=0.08$ (EtOAc). $[\alpha]^{24}{ }_{D}=-32\left(c=0.5\right.$ in $\left.\mathrm{CHCl}_{3}\right) ; \mathbf{I R}\left(\right.$ neat, $\left.\mathrm{cm}^{-1}\right): v_{\max } 3670,3392(\mathrm{br})(\mathrm{OH}), 2971,2920(\mathrm{C}-\mathrm{H}), 1683$ $(\mathrm{C}=\mathrm{O})$;

${ }^{1} \mathrm{H}$ NMR $\left(500 \mathrm{MHz}, \mathrm{CDCl}_{3}\right) \delta 5.17(\mathrm{~s}, 1 \mathrm{H}, \mathrm{H}-7), 3.86(\mathrm{~d}, J=2.4 \mathrm{~Hz}, 1 \mathrm{H}, \mathrm{H}-6), 3.83(\mathrm{~d}, J=3.1 \mathrm{~Hz}, 1 \mathrm{H}, \mathrm{H}-$ 19), 3.39 (s, 1H, H-14), 3.02 (s, 3H, H-20), $2.62-2.48$ (m, 2H, H-2), 1.55 (s, 3H, H-18), 1.51 (s, 3H, H12), 1.01 (s, 9H, H-15, H-16, H-17). ${ }^{13} \mathrm{C}$ NMR (126 MHz, CDCl $\left.{ }_{3}\right) \delta 172.9$ (C-9), 171.8 (C-3), 81.4 (C-7), 70.5 (C-5), 66.4 (C-1), 38.0 (C-11), 31.7 (C-20), 30.0 (C-12), 27.2 (C-15, C-16, C-17), 21.8 (C-18). HRMS $\mathrm{C}_{14} \mathrm{H}_{25} \mathrm{~N}_{2} \mathrm{O}_{4}[\mathrm{M}+\mathrm{H}]^{+}$calculated: 285.1736, found: 285.1732 .

(3S,7S,8aS)-3-(tert-butyl)-7-hydroxy-7-(hydroxymethyl)-2,8a-dimethylhexahydroimidazo[1,5a]pyridine-1,5-dione $10 \mathrm{~b}$
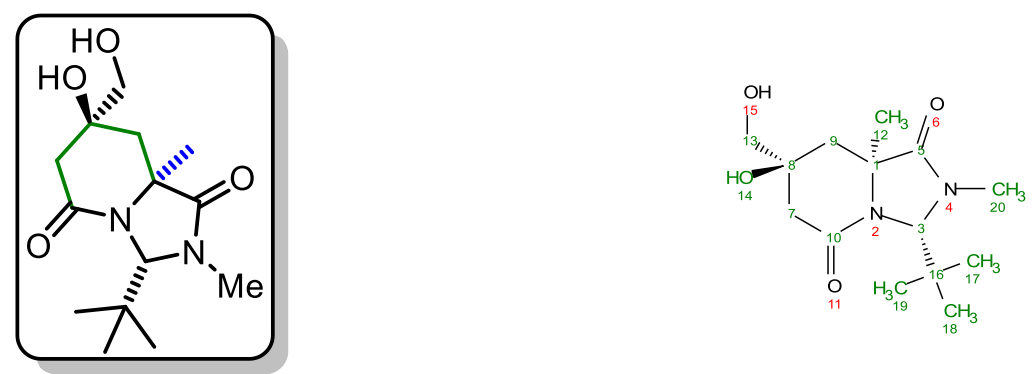

NMO (47.0 mg, $0.40 \mathrm{mmol}, 2.0$ eq.) and osmium tetroxide (126.50 $\mu \mathrm{L}, 0.02 \mathrm{mmol}, 0.02$ eq.) were added to $3 a$ ( $50.0 \mathrm{mg}, 0.20 \mathrm{mmol}, 1.0$ eq.) in dry MeCN $(1.50 \mathrm{~mL})$. The reaction stirred for $24 \mathrm{~h}$ at RT. The reaction was quenched with sat. $\mathrm{Na}_{2} \mathrm{~S}_{2} \mathrm{O}_{3}$ (aq.). The reaction mixture was extracted with EtOAc (3x). The combined organic phases were washed with brine, dried over $\mathrm{MgSO}_{4}$ and concentrated under reduced pressure. The residue was chromatographed $\left(\mathrm{SiO}_{2}\right.$, pet ether/ EtOAc 1:9) to yield the title compound as an off-white solid ( $49 \mathrm{mg}, 86 \%$ yield).

$\mathbf{R}_{f}=0.11$ (EtOAC). $[\alpha]^{24}=-60\left(c=1\right.$ in $\left.\mathrm{CHCl}_{3}\right) ; \mathbf{I R}$ (neat, $\left.\mathrm{cm}^{-1}\right): v_{\max }$ 3679, 3395 (br) (OH), 2973, 2918 (C-H), 1684 (C=O); ${ }^{1} \mathrm{H}$ NMR (500 MHz, CDCl $) \delta 5.22$ (s, 1H, H-3), 4.11 (br. s, 1H, H-14), 3.57 (q, J = $11.2 \mathrm{~Hz}, 2 \mathrm{H}, \mathrm{H}-7), 2.98(\mathrm{~s}, 3 \mathrm{H}, \mathrm{H}-20), 2.52(\mathrm{~s}, 2 \mathrm{H}, \mathrm{H}-13), 2.21$ (d, J = 14.8 Hz, 1H, H-9), 1.88 (d, J = 14.7 $\mathrm{Hz}, 1 \mathrm{H}, \mathrm{H}-9$ ), 1.52 (s, 3H, H-12), 1.01 (s, 9H, H-17, H-18, H-19). ${ }^{13} \mathrm{C}$ NMR (126 MHz, CDCl $) \delta 174.2$ (C5), 171.8 (C-10), 80.8 (C-3), 73.2 (C-1), 70.3 (C-7), 61.2 (C-8), 44.1 (C-9), 42.0 (C-13), 37.8 (C-16), 31.6 (C-20), 27.4 (C-17, C-18, C-19), $24.7(\mathrm{C}-12)$. HRMS $\mathrm{C}_{14} \mathrm{H}_{24} \mathrm{~N}_{2} \mathrm{NaO}_{4}[\mathrm{M}+\mathrm{Na}]^{+}$calculated: 307.162828, found: 307.161545 . 


\section{Hydrogenated derivatisations}

Synthesis of (3S,8aS)-3-(tert-butyl)-2,8a-dimethylhexahydroimidazo[1,5-a]pyridine-1,5-dione 11a

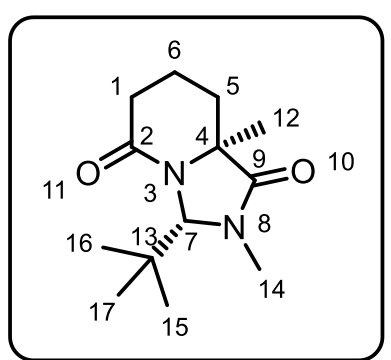

A mixture of (3S,8aS)-3-(Tert-butyl)-2,8a-dimethyl-2,3,8,8atetrahydroimidazo[1,5-a]pyridine-1,5-dione $3 a(30.0 \mathrm{mg}, 0.127 \mathrm{mmol}, 1.0$ eq.), $10 \%$ palladium on carbon $(8.52 \mathrm{mg}, 0.080 \mathrm{mmol}, 0.63$ eq.), acetic acid ( 1 drop) and methanol $(6 \mathrm{~mL})$ was stirred at RT under $\mathrm{H}_{2}(1 \mathrm{~atm})$ for $24 \mathrm{~h}$. The reaction mixture was filtered through celite, washing with DCM and then concentrated under reduced pressure. The residue was extracted with EtOAc (x3) and sat. $\mathrm{NaHCO}_{3}$ (aq.). The combined organic phases were washed with brine, dried over $\mathrm{MgSO}_{4}$ and concentrated under reduced pressure to yield the crude title compound as a light-yellow oil (30.0 $\mathrm{mg}$, quantitative yield).

$[\alpha]^{24}{ }_{D}=-36\left(c=0.5\right.$ in $\left.\mathrm{CHCl}_{3}\right)$; IR (neat, $\left.\mathrm{cm}^{-1}\right): v_{\max }$ 2966, $2924(\mathrm{C}-\mathrm{H}), 1706,1665$ (C=O); ${ }^{1} \mathbf{H}$ NMR (500 $\left.\mathrm{MHz}, \mathrm{CDCl}_{3}\right) \delta 5.25$ (s, 1H, H-7), 2.99 (s, 3H, H-14), 2.55 (ddd, J=17.5, 10.3, $4.0 \mathrm{~Hz}, 1 \mathrm{H}, \mathrm{H}-1$ ), 2.42 (ddd, J = 17.6, 9.8, 6.2 Hz, 1H, H-1), $2.12-2.07(\mathrm{~m}, 1 \mathrm{H}, \mathrm{H}-5), 2.06-1.99(\mathrm{~m}, 1 \mathrm{H}, \mathrm{H}-6), 1.95-1.86(\mathrm{~m}$, $1 \mathrm{H}, \mathrm{H}-5), 1.52$ (d, J = 0.7 Hz, 3H, H-12), 1.36 (td, J = 12.7, 12.2, $5.5 \mathrm{~Hz}, 1 \mathrm{H}, \mathrm{H}-5), 1.03(\mathrm{~s}, 9 \mathrm{H}, \mathrm{H}-15+\mathrm{H}-$ $16+\mathrm{H}-17) ;{ }^{13} \mathrm{C}$ NMR (126 MHz, CDCl 3 ) $\delta 174.3$ (C-9), 172.1 (C-2), 80.8 (C-7), 62.2 (C-4), 37.6 (C-13), 33.4 (C-5), 31.3 (C-14), 30.0 (C-1), 27.4 (C-15 + C-16 + C-17), 22.1 (C-12), 15.6 (C-6); HRMS $\mathrm{C}_{13} \mathrm{H}_{22} \mathrm{~N}_{2} \mathrm{O}_{2}$ $[\mathrm{M}+\mathrm{H}]^{+}$calculated: 239.1754 , found: 239.1748 .

Synthesis of (3R,8aS)-3-(tert-butyl)-2,8a-dimethylhexahydroimidazo[1,5-a]pyridin-1(5H)-one 12

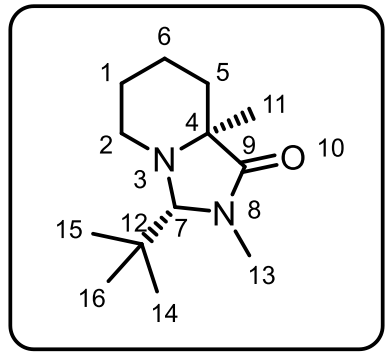

(3S,8aS)-3-(tert-butyl)-2,8a-dimethylhexahydroimidazo[1,5-a]pyridine1,5-dione 11a (67.3 mg, $0.282 \mathrm{mmol}, 1.0$ eq.) was dissolved in THF (1.88 $\mathrm{mL}, 0.15 \mathrm{M})$ and cooled in an ice-water bath. $\mathrm{BH}_{3} \mathrm{SMe}_{2}(2 \mathrm{M}$ in THF, 0.27 $\mathrm{mL}, 0.541 \mathrm{mmol}, 1.9$ eq.) was added and the reaction mixture stirred for $17 \mathrm{~h}$ at RT. The reaction was quenched with EtOH at $-2{ }^{\circ} \mathrm{C}$ and left to stir for $1 \mathrm{~h}$. The reaction mixture was concentrated under reduced pressure. The residue was chromatographed $\left(\mathrm{SiO}_{2}\right.$, hexane/ EtOAc 89:11) to yield the title compound as a colourless oil $\left(43.8 \mathrm{mg}, 70 \%\right.$ yield). $\mathbf{R}_{f}=0.21$ (9:1 hexane/ EtOAc). ${ }^{1} \mathbf{H}$ NMR $\left(500 \mathrm{MHz}, \mathrm{CDCl}_{3}\right) \delta 3.85(\mathrm{~s}, 1 \mathrm{H}, \mathrm{H}-7), 2.93(\mathrm{~s}, 3 \mathrm{H}, \mathrm{H}-13), 2.92-2.72(\mathrm{~m}, 2 \mathrm{H}, \mathrm{H}-2), 1.68-1.51(\mathrm{~m}, 4 \mathrm{H}$, $\mathrm{H}-1+\mathrm{H}-5+\mathrm{H}-6), 1.43-1.27(\mathrm{~m}, 2 \mathrm{H}, \mathrm{H}-1+\mathrm{H}-6), 1.25$ (s, 3H, H-11), 0.92 (s, 9H, H-14 + H-15 + H-16); ${ }^{13} \mathrm{C}$ NMR (126 MHz, CDCl ${ }_{3}$ ) $\delta 177.7$ (C-9), 86.5 (C-7), 61.3 (C-4), 45.2 (C-2), 37.9 (C-12), 31.5 (C-13), 30.6 (C-5), 26.2 (C-14 + C-15 + C-16), 24.8 (C-11), 19.2 (C-1), 17.1 (C-6). HRMS $\mathrm{C}_{13} \mathrm{H}_{24} \mathrm{~N}_{2} \mathrm{O}[\mathrm{M}+\mathrm{H}]^{+}$calculated: 225.1889, found: 225.1888 . 

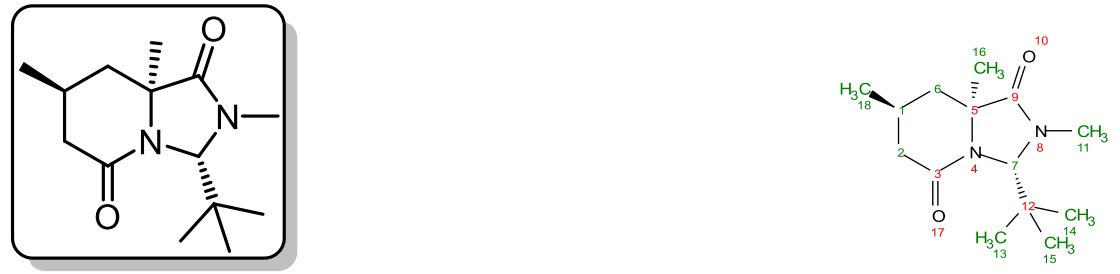

A mixture of (3S,8aS)-3-(tert-butyl)-2,7,8a-trimethyl-2,3,8,8a-tetrahydroimidazo[1,5-a]pyridine-1,5dione $3 \mathrm{~h}$ (125.0 mg, $0.500 \mathrm{mmol}, 1.0$ eq.), $10 \%$ palladium on carbon ( $33.5 \mathrm{mg}, 0.31 \mathrm{mmol}, 0.63 \mathrm{eq}$.), acetic acid (1 drop) and methanol ( $25 \mathrm{~mL}, 0.02 \mathrm{M}$ ) was stirred at RT under $\mathrm{H}_{2}(1 \mathrm{~atm})$ for $24 \mathrm{~h}$. The reaction mixture was filtered through celite, washing with $\mathrm{DCM}$ and then concentrated under reduced pressure. The residue was extracted with EtOAc (x3) and sat. $\mathrm{NaHCO}_{3}$ (aq.). The combined organic phases were washed with brine, dried over $\mathrm{MgSO}_{4}$ and concentrated under reduced pressure to yield the crude title compound as a Beige solid (107.0 mg, 85\%). $\mathbf{R}_{\boldsymbol{f}}=0.33$ (pet.ether/EtOAC $=4: 1) .[\alpha]^{20}{ }_{D}=-112\left(c=1\right.$ in $\left.\mathrm{CHCl}_{3}\right) ; \mathbf{m p} 58-60{ }^{\circ} \mathrm{C} ; \mathbf{I R}$ (neat, $\left.\mathrm{cm}^{-1}\right): v_{\max } 2965,2920$ (C-H), 1703, 1668 (C=O); ${ }^{1} \mathrm{H}$ NMR (500 MHz, CDCl 3 ) $\delta 5.19$ (s, 1H, H-7), 2.95 (s, 3H, H-11), 2.73 (dd, J = $17.8,7.2 \mathrm{~Hz}, 1 \mathrm{H}, \mathrm{H}-2), 2.36-2.23(\mathrm{~m}, 1 \mathrm{H}, \mathrm{H}-1), 2.09-2.05(\mathrm{~m}, 1 \mathrm{H}, \mathrm{H}-2), 1.96(\mathrm{dd}, J=17.8,7.2 \mathrm{~Hz}, 1 \mathrm{H}$, $\mathrm{H}-6), 1.49$ (s, 3H, H-16), $1.09-1.00$ (m, 4H, H-6, H-18), 1.00 (s, 9H, H-13, H-14, H-15). ${ }^{13} \mathrm{C}$ NMR (126 $\left.\mathrm{MHz}, \mathrm{CDCl}_{3}\right) \delta 174.0,172.2,80.8,61.8,41.8,39.0,37.2,31.0,27.3,23.0,22.9,22.4$. HRMS $\mathrm{C}_{14} \mathrm{H}_{25} \mathrm{~N}_{2} \mathrm{O}_{2}$ $[\mathrm{M}+\mathrm{H}]^{+}$calculated: 253.1910 , found: 253.1901 .

(3S,8aS)-3-(tert-butyl)-7-(4-fluorophenyl)-2,8a-dimethylhexahydroimidazo[1,5-a]pyridine-1,5dione 11c

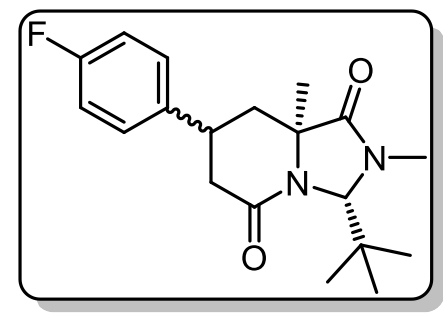

A mixture of

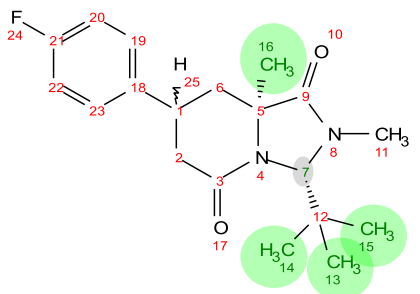

(3S,8aS)-3-(tert-butyl)-7-(4-fluorophenyl)-2,8a-dimethyl-2,3,8,8atetrahydroimidazo[1,5-a]pyridine-1,5-dione $3 c(145.0 \mathrm{mg}, 0.44 \mathrm{mmol}, 1.0 \mathrm{eq}),. 10 \%$ palladium on carbon (30 mg, $0.69 \mathrm{mmol}, 0.65$ eq.), acetic acid (1 drop) and methanol ( $22 \mathrm{~mL}, 0.02 \mathrm{M}$ ) was stirred at $\mathrm{RT}$ under $\mathrm{H}_{2}(1 \mathrm{~atm})$ for $24 \mathrm{~h}$. The reaction mixture was filtered through celite, washing with DCM and then concentrated under reduced pressure. The residue was extracted with EtOAc $(x 3)$ and sat. $\mathrm{NaHCO}_{3}$ (aq.). The combined organic phases were washed with brine, dried over $\mathrm{MgSO}_{4}$ and concentrated under reduced pressure to yield the crude title compound as a Beige solid $(107.0 \mathrm{mg}$,

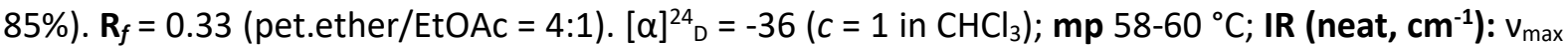
2965, 2920 (C-H), 1703, 1668 (C=O); ${ }^{1} \mathrm{H}$ NMR (500 MHz, CDCl $) \delta 7.23-7.15$ (m, 1.54H, H-20, H-22), $7.15-7.11(\mathrm{~m}, 0.46 \mathrm{H}, \mathrm{H}-20, \mathrm{H}-22), 7.10-7.06(\mathrm{~m}, 0.46 \mathrm{H}, \mathrm{H}-19, \mathrm{H}-23), 7.04-6.95(\mathrm{~m}, 1.54 \mathrm{H}, \mathrm{H}-19$, $\mathrm{H}-23$ ), 5.27 (s, 0.23H, H-7), $5.22(\mathrm{~s}, 0.77 \mathrm{H}, \mathrm{H}-7$ ), 3.44 (tdd, J = 12.1, 7.3, $4.5 \mathrm{~Hz}, 0.23 \mathrm{H}, \mathrm{H}-1$ ), 3.14 (ddt, $J=12.1,7.3,4.5 \mathrm{~Hz}, 0.77 \mathrm{H}, \mathrm{H}-1), 3.01\left(\mathrm{~s}, 3 \mathrm{H}, \mathrm{H}-11\right.$ major + minor), $3.00\left(\mathrm{~s}, 0.23 \mathrm{H}, \mathrm{H}-2_{\mathrm{B}}\right.$ minor), $2.70-$ $2.58\left(\mathrm{~m}, 1.54 \mathrm{H}, \mathrm{H}-2_{\mathrm{A}}\right.$ major + $\mathrm{H}-2_{\mathrm{B}}$ major), $2.44\left(\mathrm{dd}, J=17.8,7.3 \mathrm{~Hz}, 0.23 \mathrm{H}, \mathrm{H}-2_{\mathrm{A}}\right.$ minor), $2.36-2.27$ (m, 1H, H-6 B major + minor), 2.18 (dd, $J=14.5,6.4 \mathrm{~Hz}, 1 \mathrm{H}, \mathrm{H}-6 \mathrm{~A}$ major + minor), $1.63(\mathrm{~s}, 0.69 \mathrm{H}, \mathrm{H}-$ 16), 1.54 (s, 2.31H, H-16), 1.09 (s, 2.07H, H-13, H-14, H-15), 1.05 (s, 6.93H, H-13, H-14, H-15). ${ }^{13} \mathrm{C}$ NMR $\left(126 \mathrm{MHz}, \mathrm{CDCl}_{3}\right) \delta 173.9,173.7,172.5,171.7,162.7,162.6,160.8,160.7,140.0,139.9,139.2$, 139.1, 128.2, 128.2, 128.2, 128.1, 115.9, 115.8, 115.7, 115.7, 81.0, 80.7, 62.4, 61.7, 41.8, 41.3, 39.0, $38.5,37.7,37.4,35.7,33.6,31.3,31.2,27.5,27.4,27.4,26.3,23.0$. HRMS $\mathrm{C}_{19} \mathrm{H}_{26} \mathrm{FN}_{2} \mathrm{O}_{2}[\mathrm{M}+\mathrm{H}]^{+}$ calculated: 333.1972 , found: 333.1967 . 
Synthesis of $S$-((3S,7S,8aS)-3-(tert-butyl)-2,8a-dimethyl-1,5-dioxooctahydroimidazo[1,5-a]pyridin7 -yl) ethanethioate $9 a$

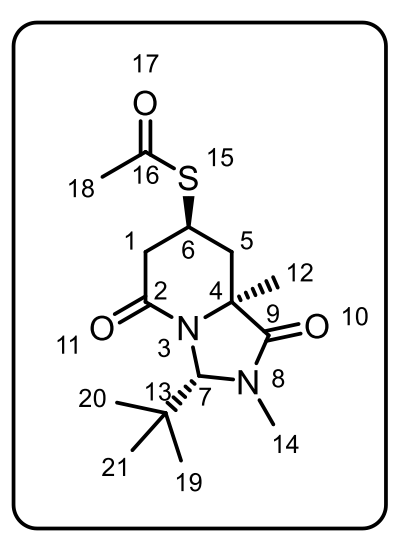

(3S,8aS)-3-(Tert-butyl)-2,8a-dimethyl-2,3,8,8a-tetrahydroimidazo[1,5a]pyridine-1,5-dione $3 a$ (100 $\mathrm{mg}, 0.423 \mathrm{mmol}, 1.0$ eq.) was dissolved in dry MeCN (5.3 mL). Aluminium trichloride (169 mg, $1.27 \mathrm{mmol}, 3$ eq.) and trifluoroacetic acid ( $31.4 \mu \mathrm{L}, 0.423 \mathrm{mmol}, 1.0$ eq.) were added and the reaction stirred in an oil bath at $100{ }^{\circ} \mathrm{C}$ for $30 \mathrm{~min}$. Potassium thioacetate (241.8 $\mathrm{mg}, 2.12 \mathrm{mmol}, 5.0$ eq.) was added and the reaction mixture stirred in an oil bath at $100{ }^{\circ} \mathrm{C}$ for $24 \mathrm{~h}$. Over the course of the reaction the colour of the reaction mixture transitioned from orange to dark brown. The reaction was quenched with sat. $\mathrm{NaHCO}_{3}$ (aq.) then extracted with EtOAc (x3). The combined organic phases were washed with brine, dried over $\mathrm{MgSO}_{4}$ and concentrated under reduced pressure. The residue was chromatographed $\left(\mathrm{SiO}_{2}\right.$, pet ether/ EtOAc 4:6) to yield the title compound as a brown oil (116 $\mathrm{mg}, 87 \%$ yield). $\mathbf{R}_{f}=0.28$ (pet.ether/EtOAc $\left.=4: 6\right) .[\alpha]_{\mathrm{D}}^{23}=-28\left(c=1\right.$ in $\left.\mathrm{CHCl}_{3}\right) ; \mathbf{I R}$ (neat, $\left.\mathbf{c m}^{-1}\right): v_{\max }$ 2971, 2923 (C-H), 1703, 1665 (C=O); ${ }^{1} \mathrm{H}$ NMR (500 MHz, CDCl $) \delta 5.22$ (s, 1H, H-7), $4.12-4.05$ (m, 1H, $\mathrm{H}-6), 3.13$ (dd, J=18.3, 9.0 Hz, 1H, H-5), 2.99 (s, 3H, H-14), 2.42 (dd, J=18.3, 7.8 Hz, 1H, H-5), 2.36 (dd, $J=13.0,4.5 \mathrm{~Hz}, 1 \mathrm{H}, \mathrm{H}-1), 2.33(\mathrm{~s}, 3 \mathrm{H}, \mathrm{H}-18), 1.65-1.61(\mathrm{~m}, 3 \mathrm{H}, \mathrm{H}-12), 1.50(\mathrm{t}, J=13.0 \mathrm{~Hz}, 1 \mathrm{H}, \mathrm{H}-$ 1), $1.04(\mathrm{~s}, 9 \mathrm{H}, \mathrm{H}-19+\mathrm{H}-20+\mathrm{H}-21) ;{ }^{13} \mathrm{C}$ NMR $\left(126 \mathrm{MHz}, \mathrm{CDCl}_{3}\right) \delta 194.8$ (C-9), 173.1 (C-2), 170.0 (C16), 81.2 (C-7), 61.4 (C-4), 39.5 (C-1), 38.0 (C-5), 37.4 (C-13), 32.4 (C-6), 31.2 (C-14), 30.7 (C-18), 27.5 (C-19+C-20 + C-21), $23.2(\mathrm{C}-12) ;$ HRMS $\mathrm{C}_{15} \mathrm{H}_{24} \mathrm{~N}_{2} \mathrm{O}_{3} \mathrm{~S}[\mathrm{M}+\mathrm{H}]^{+}$calculated:313.1580, found: 313.1576.

Synthesis of $S$-((3S,7R,8aS)-3-(tert-butyl)-2,8a-dimethyl-1,5-dioxooctahydroimidazo[1,5-a]pyridin7 -yl) ethanethioate $9 b$

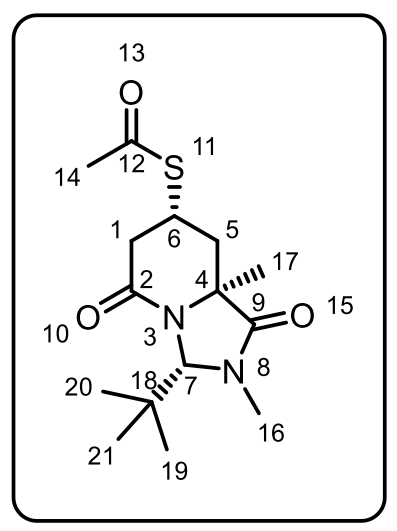

(3S,8aS)-3-(Tert-butyl)-2,8a-dimethyl-2,3,8,8a-tetrahydroimidazo[1,5a]pyridine-1,5-dione $3 a(50.0 \mathrm{mg}, 0.212 \mathrm{mmol}, 1.0$ eq.) in thioacetic acid $(0.25 \mathrm{~mL})$ was refluxed in an oil bath at $100{ }^{\circ} \mathrm{C}$ for $24 \mathrm{~h}$. The colour of the reaction mixture transitioned from orange to dark brown. The reaction was quenched with sat. $\mathrm{NaHCO}_{3}$ (aq.) then extracted with EtOAc (x3). The combined organic phases were washed with brine, dried over $\mathrm{MgSO}_{4}$ and concentrated under reduced pressure. The residue was chromatographed ( $\mathrm{SiO}_{2}$, pet ether/ EtOAc 1:1) to yield the title compound as a brown oil (34.7 mg, 55\% yield). $\mathbf{R}_{f}=0.11$ (pet.ether/EtOAc $\left.=1: 1\right) .[\alpha]_{\mathrm{D}}^{23}=-4(c=0.5$ in $\mathrm{CHCl}_{3}$ ); IR (neat, $\mathrm{cm}^{-1}$ ): $\mathrm{V}_{\max }$ 2988, 2901 (C-H), 1702 (C=O); ${ }^{1} \mathbf{H}$ NMR (400 $\left.\mathrm{MHz}, \mathrm{CDCl}_{3}\right) \delta 5.20(\mathrm{~s}, 1 \mathrm{H}, \mathrm{H}-7$ ), 4.05 (qd, $J=9.3,1.6 \mathrm{~Hz}, 1 \mathrm{H}, \mathrm{H}-6), 2.99$ (s, 3H, H-16), 2.94 (dd, J = 15.1, 9.0 Hz, 1H, H-5), 2.49 (dd, J = 15.1, 9.3 Hz, 1H, H-5), 2.34 (s, 3H, H-14), 2.22 (dd, J = 15.1, $1.6 \mathrm{~Hz}$, $1 \mathrm{H}, \mathrm{H}-1$ ), 2.04 (dd, J = 15.1, 9.0 Hz, 1H, H-1), 1.60 (s, 3H, H-17), 1.01 (s, 9H, H-19+ H-20 + H-21); ${ }^{13} \mathrm{C}$ NMR (101 MHz, CDCl ${ }_{3}$ ) $\delta 194.1$ (C-9), 173.3 (C-2), 170.3 (C-12), 80.4 (C-7), 62.7 (C-4), 41.1 (C-1), 38.1 (C-18), 37.5 (C-5), 33.0 (C-6), 31.5 (C-16), 30.5 (C-14), 27.1 (C-19 + C-20 + C-21), 22.5 (C-17); HRMS $\mathrm{C}_{15} \mathrm{H}_{24} \mathrm{~N}_{2} \mathrm{O}_{3} \mathrm{~S}[\mathrm{M}+\mathrm{H}]^{+}$calculated: 313.1580 , found: 313.1577 . 


\section{$\underline{\text { Hydrolysed products }}$}

(S)-2,4-dimethyl-6-oxo-1,2,3,6-tetrahydropyridine-2-carboxylic acid 13a
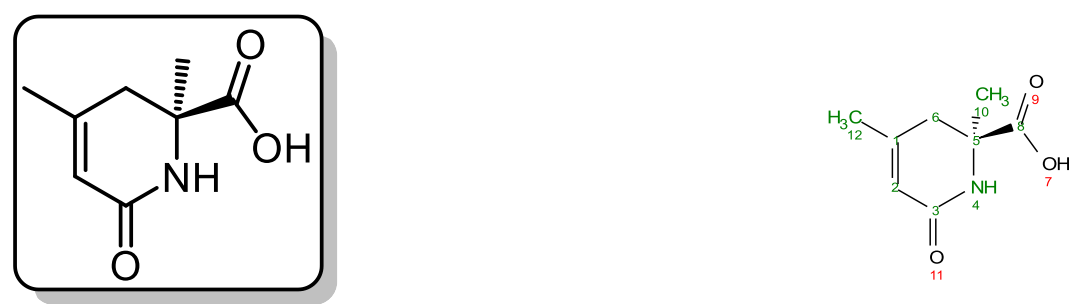

Alkylated lactam $3 \mathrm{~h}(79 \mathrm{mg}, 0.16 \mathrm{mmol})$ was dissolved in a mixture of TFA $-6.0 \mathrm{M} \mathrm{HCl}$ (1:4 ratio) $(6.39 \mathrm{~mL}, 0.05 \mathrm{M})$ in a microwave vial. The reaction was heated in a microwave reactor at $170^{\circ} \mathrm{C}(12$ bar) for $4 \mathrm{~h}$. The reaction mixture was concentrated under reduced pressure. The crude material purified by flash column chromatography to yield the title compound $(42.5 \mathrm{mg}, 80 \%)$ as a yellowish white oil. $\mathbf{R}_{\boldsymbol{f}} 0.37$ (pet.ether/EtOAc 3:1, $1 \%$ formic acid); $[\alpha]^{24}{ }_{\mathrm{D}}=+28(\mathrm{c}=0.5 \mathrm{in} \mathrm{MeOH})$; IR vmax = 3231 (OH), 2925 (C-H), 1705 (C=O acid), 1629 (C=O amide); ${ }^{1} \mathbf{H}$ NMR (500 MHz, CDCl $\left.{ }_{3}\right) 8.89$ (s, $1 \mathrm{H}$, $\mathrm{H}-4$ ), 5.72 (s, 1H, H-2), 2.76 (d, J = $17.8 \mathrm{~Hz}, 1 \mathrm{H}, \mathrm{H}-6), 2.43$ (d, J = $17.8 \mathrm{~Hz}, 1 \mathrm{H}, \mathrm{H}-6), 1.97$ (s, 3H, H-12), 1.55 (s, 3H, H-10). ${ }^{13} \mathrm{C}$ NMR (126 MHz, CDCl 3 ) $\delta 177.3$ (C-8), 167.5 (C-3), 152.3 (C-1), 118.3 (C-2), 57.7 (C-5), 37.6 (C-6), 25.0 (C-10). HRMS $\mathrm{C}_{8} \mathrm{H}_{12} \mathrm{NO}_{3}[\mathrm{M}+\mathrm{H}]+$ calculated: 170.0739 , found 170.0744 .

\section{(2S,4S)-2,4-dimethyl-6-oxopiperidine-2-carboxylic acid 13b}
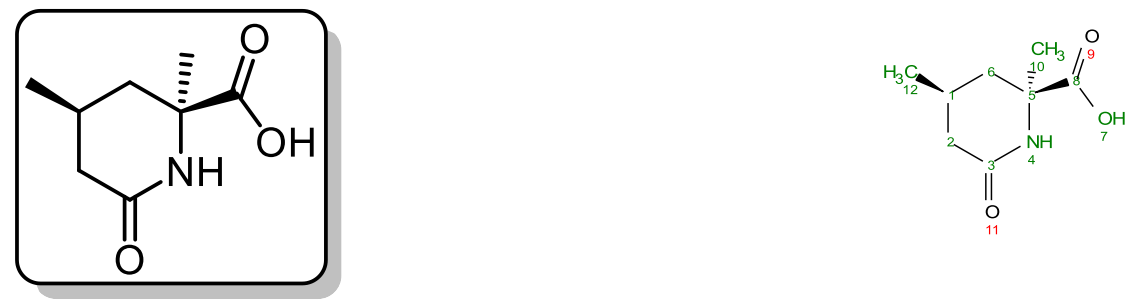

Alkylated lactam $11 \mathrm{~h}(96 \mathrm{mg}, 0.38 \mathrm{mmol}$ ) was dissolved in a mixture of TFA - $6.0 \mathrm{M} \mathrm{HCl}$ (1:4 ratio) $(7.6 \mathrm{~mL}, 0.05 \mathrm{M})$ in a microwave vial. The reaction was heated in a microwave reactor at $170{ }^{\circ} \mathrm{C}(12$ bar) for $4 \mathrm{~h}$. The reaction mixture was concentrated under reduced pressure. The crude material was washed by $\mathrm{Et}_{2} \mathrm{O}(20 \mathrm{ml})$ to yield the title compound $(57 \mathrm{mg}, 88 \%)$ as a yellow oil. $\mathbf{R}_{\boldsymbol{f}} 0.40$ (pet.ether/EtOAc 3:1, 1\% formic acid); $[\alpha]^{24}{ }_{D}=-28$ (c=0.25 in MeOH); IR vmax = $3231(\mathrm{OH}), 2925$ (CH), 1705 (C=O acid), 1629 (C=O amide); ${ }^{1} \mathrm{H}$ NMR ( $\left.500 \mathrm{MHz}, \mathrm{CDCl}_{3}\right) \delta 8.75$ (s, $1 \mathrm{H}, \mathrm{H}-7$ ), 5.86 (br. s, $1 \mathrm{H}$, $\mathrm{H}-4$ ), 2.45 (ddd, $J=18.1,5.6,1.9 \mathrm{~Hz}, 1 \mathrm{H}, \mathrm{H}-2$ ), $2.21-2.02$ (m, 2H, H-2, H-1), 1.83 (dd, $J=18.1,11.4$ $\mathrm{Hz}, 1 \mathrm{H}, \mathrm{H}-6), 1.54$ (s, 4H, H-6, H-10), 1.04 (d, J = 6.4 Hz, 3H, H-12). ${ }^{13} \mathrm{C} \mathrm{NMR} \mathrm{(126} \mathrm{MHz,} \mathrm{CDCl} 3$ ) $\delta 177.4$ (C-8), 173.6 (C-3), 58.7 (C-5), 38.7 (C-2), 38.1 (C-6), 27.1 (C-10), 23.5 (C-1), 21.5 (C-12). HRMS $\mathrm{C}_{8} \mathrm{H}_{14} \mathrm{NO}_{3}[\mathrm{M}+\mathrm{H}]+$ calculated: 172.0968 , found 172.0977 . 

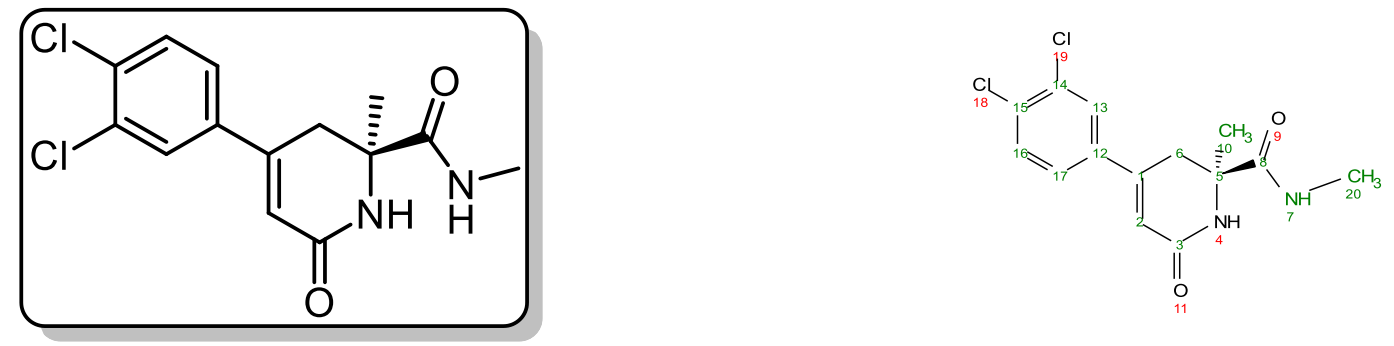

Alkylated lactam $\mathbf{3 d}(46 \mathrm{mg}, 0.16 \mathrm{mmol})$ was dissolved in a mixture of TFA $-6.0 \mathrm{M} \mathrm{HCl}$ (1:4 ratio) (3.2 $\mathrm{mL}, 0.05 \mathrm{M}$ ) in a microwave vial. The reaction was heated in a microwave reactor at $175{ }^{\circ} \mathrm{C}$ (12 bar) for $4 \mathrm{~h}$. The reaction mixture was concentrated under reduced pressure. The crude material was washed by $\mathrm{Et}_{2} \mathrm{O}(20 \mathrm{ml})$ to yield the title compound $(23 \mathrm{mg}, 75 \%)$ as a yellow oil. $\mathbf{R}_{\boldsymbol{f}} 0.32$ (pet.ether/EtOAc 3:1, 1\% formic acid); $[\alpha]^{24}{ }_{\mathrm{D}}=+28$ (c=0.5 in $\left.\mathrm{CHCl}_{3}\right)$; IR vmax = $3231(\mathrm{OH}), 3126$ (CH) 2925 (C-H), 1705 (C=O acid), 1629 (C=O amide); ${ }^{1} \mathrm{H}$ NMR (500 MHz, CDCl $\left.{ }_{3}\right) \quad 7.63-7.55$ (m, 1H, HAr), 7.49 (d, J = 8.4 Hz, 1H, H-Ar), 7.35 (dd, J = 8.4, $2.2 \mathrm{~Hz}, 1 \mathrm{H}, \mathrm{H}-\mathrm{Ar}$ ), 6.38 (br. s, $1 \mathrm{H}, \mathrm{H}-4), 6.21$ (s, $1 \mathrm{H}$, $\mathrm{H}-2$ ), $3.80-3.67(\mathrm{~m}, 3 \mathrm{H}, \mathrm{H}-20), 3.28(\mathrm{~d}, J=16.7 \mathrm{~Hz}, 1 \mathrm{H}, \mathrm{H}-6), 2.75(\mathrm{~d}, J=16.7 \mathrm{~Hz}, 1 \mathrm{H}, \mathrm{H}-6), 1.59$ (s, 3H, H-10). ${ }^{13}$ C NMR (126 MHz, CDCl 3 ) $\delta 174.0$ (C-8), 166.4 (C-3), 147.7 (C-1), 137.3 (C-12), 134.3 (C14), 133.4 (C-15), 131.0 (C-16), 128.2 (C-13), 125.4 (C-17), 119.9 (C-2), 58.9 (C-5), 53.4 (C-20), 36.2 (C6), 25.9 (C-10). $\mathrm{HRMS} \mathrm{C}_{14} \mathrm{H}_{15} \mathrm{ClN}_{2} \mathrm{O}_{2}[\mathrm{M}+\mathrm{H}]+$ calculated: 313.0432 , found 313.0440.

(S)-4-(4'-bromo-[1,1'-biphenyl]-4-yl)-2-methyl-6-oxo-1,2,3,6-tetrahydropyridine-2-carboxylic acid 13d
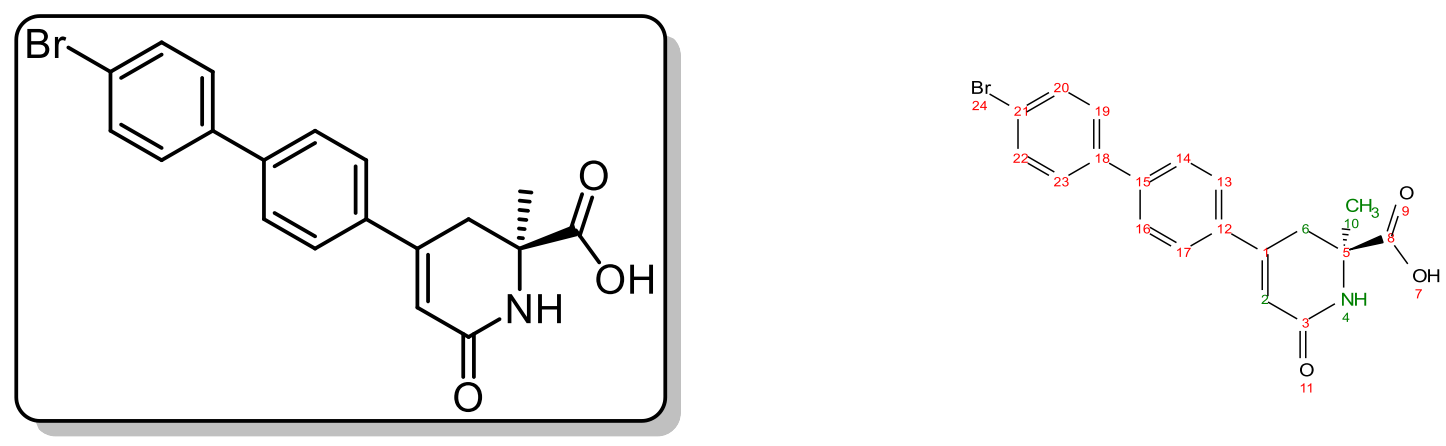

Alkylated lactam $3 e(71 \mathrm{mg}, 0.15 \mathrm{mmol})$ was dissolved in a mixture of TFA $-6.0 \mathrm{M} \mathrm{HCl}$ (1:4 ratio) (3.0 $\mathrm{mL}, 0.05 \mathrm{M}$ ) in a microwave vial. The reaction was heated in a microwave reactor at $170{ }^{\circ} \mathrm{C}(14$ bar) for $4 \mathrm{~h}$. The reaction mixture was concentrated under reduced pressure. The crude material purified by flash column chromatography to yield the title compound (41 $\mathrm{mg}, 70 \%)$ as a beige semi-solid. $\mathbf{R}_{f}$ 0.12 (pet.ether/EtOAc 3:1, 1\% formic acid); $[\alpha]^{24}=+44$ (c = 1 in MeOH); IR vmax = $3231(\mathrm{OH}), 3126$ (C-H) 2925 (C-H), 1705 (C=O acid), 1629 (C=O amide); $\left.{ }^{1} \mathrm{H} \mathrm{NMR} \mathrm{(500} \mathrm{MHz,} \mathrm{CDCl}\right) \delta 7.63-7.55$ (m, $1 \mathrm{H}$, $\mathrm{H}-\mathrm{Ar}$ ), 7.49 (d, J = 8.4 Hz, 1H, H-Ar), 7.35 (dd, J = 8.4, 2.2 Hz, 1H, H-Ar), 6.38 (br. s, $1 \mathrm{H}, \mathrm{H}-4), 6.21$ (s, $1 \mathrm{H}, \mathrm{H}-2$ ), $3.80-3.67$ (m, 3H, H-20), 3.28 (d, J = 16.7 Hz, 1H, H-6), 2.75 (d, J = 16.7 Hz, 1H, H-6), 1.59 (s, 3H, H-10). ${ }^{13} \mathrm{C}$ NMR (126 MHz, CDCl $) \delta 174.0$ (C-8), 166.4 (C-3), 147.7 (C-1), 137.3 (C-12), 134.3 (C14), 133.4 (C-15), 131.0 (C-16), 128.2 (C-13), 125.4 (C-17), 119.9 (C-2), 58.9 (C-5), 53.4 (C-20), 36.2 (C6), 25.9 (C-10). HRMS $\mathrm{C}_{19} \mathrm{H}_{17} \mathrm{BrNO}_{3}[\mathrm{M}+\mathrm{H}]+$ calculated: 387.0313 , found 387.0320. 

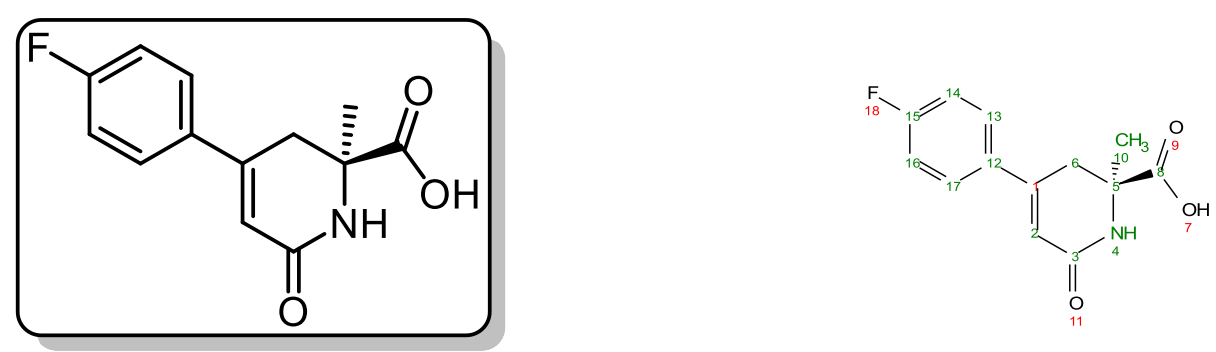

Alkylated lactam 3c $(39.2 \mathrm{mg}, 0.12 \mathrm{mmol})$ was dissolved in a mixture of TFA $-6.0 \mathrm{M} \mathrm{HCl}$ (1:4 ratio) $(2.5 \mathrm{~mL}, 0.05 \mathrm{M})$ in a microwave vial. The reaction was heated in a microwave reactor at $170{ }^{\circ} \mathrm{C}(12$ bar) for $4 \mathrm{~h}$. The reaction mixture was concentrated under reduced pressure. The crude material purified by flash column chromatography to yield the title compound $(20 \mathrm{mg}, 68 \%$ ) as a dark yellow oil. $\boldsymbol{R}_{f} 0.25$ (pet.ether/EtOAc 3:1, 1\% formic acid); $[\alpha]^{24}{ }_{\mathrm{D}}=+8\left(\mathrm{c}=0.5\right.$ in $\left.\mathrm{CHCl}_{3}\right)$; IR vmax = $3231(\mathrm{OH})$, 3126 (C-H) 2925 (C-H), 1705 (C=O acid), 1629 (C=O amide); ${ }^{1} \mathbf{H}$ NMR (500 MHz, CDCl $) \delta 9.09$ (s, 1H, $\mathrm{H}-4), 7.64-7.45$ (m, 2H, H-13, H-17), $7.19-7.03$ (m, 2H, H-14, H-16), 6.23 (s, 1H, H-2), 3.14 (dd, J = 17.4, $2.4 \mathrm{~Hz}, 1 \mathrm{H}, \mathrm{H}-6), 3.03$ (d, J = 17.4 Hz, 1H, H-6), 1.66 (s, 3H, H-10). ${ }^{13} \mathrm{C}$ NMR (126 MHz, CDCl $\left.{ }_{3}\right) \delta$ 177.4 (C-8), 167.3 (C-3), 165.0 (C-15), 163.0 (C-15), 133.4 (C-2), 133.4 (C-12), 128.3 (C-13, C-17), 128.2 (C-13, C-17), 117.4 (C-1), 116.3 (C-14, C-16), 116.1 (C-14, C-16), 57.8 (C-5), 35.0 (C-6), 25.0 (C10). HRMS $\mathrm{C}_{13} \mathrm{H}_{13} \mathrm{FNO}_{3}[\mathrm{M}+\mathrm{H}]^{+}$calculated: 250.0801 , found 250.0813. 


\section{Spectral data: ${ }^{1} \mathrm{H},{ }^{13} \mathrm{C}$ NMR and IR}

\section{S1}
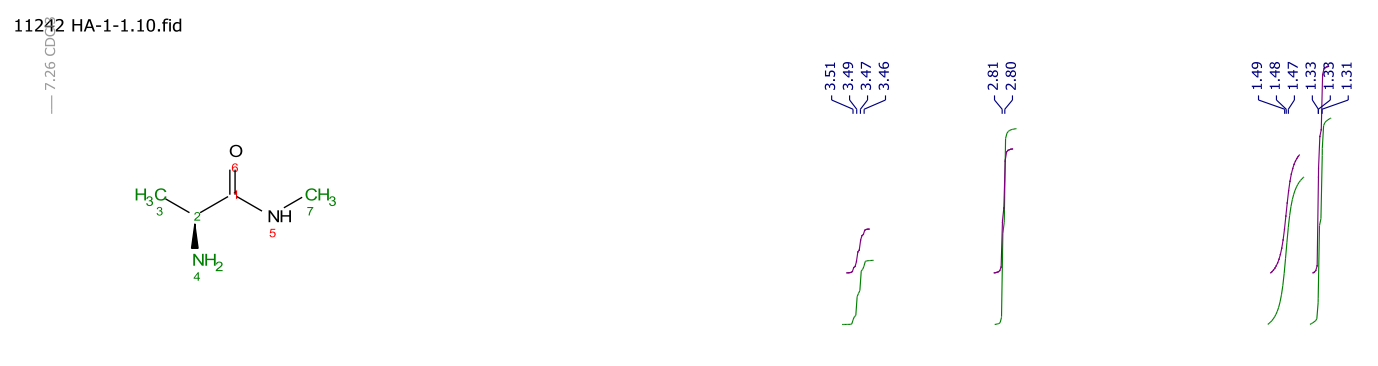

$-130000$

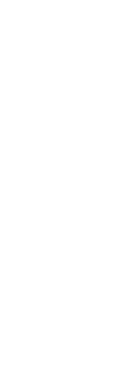

120000

110000

100000

$-90000$

$-80000$

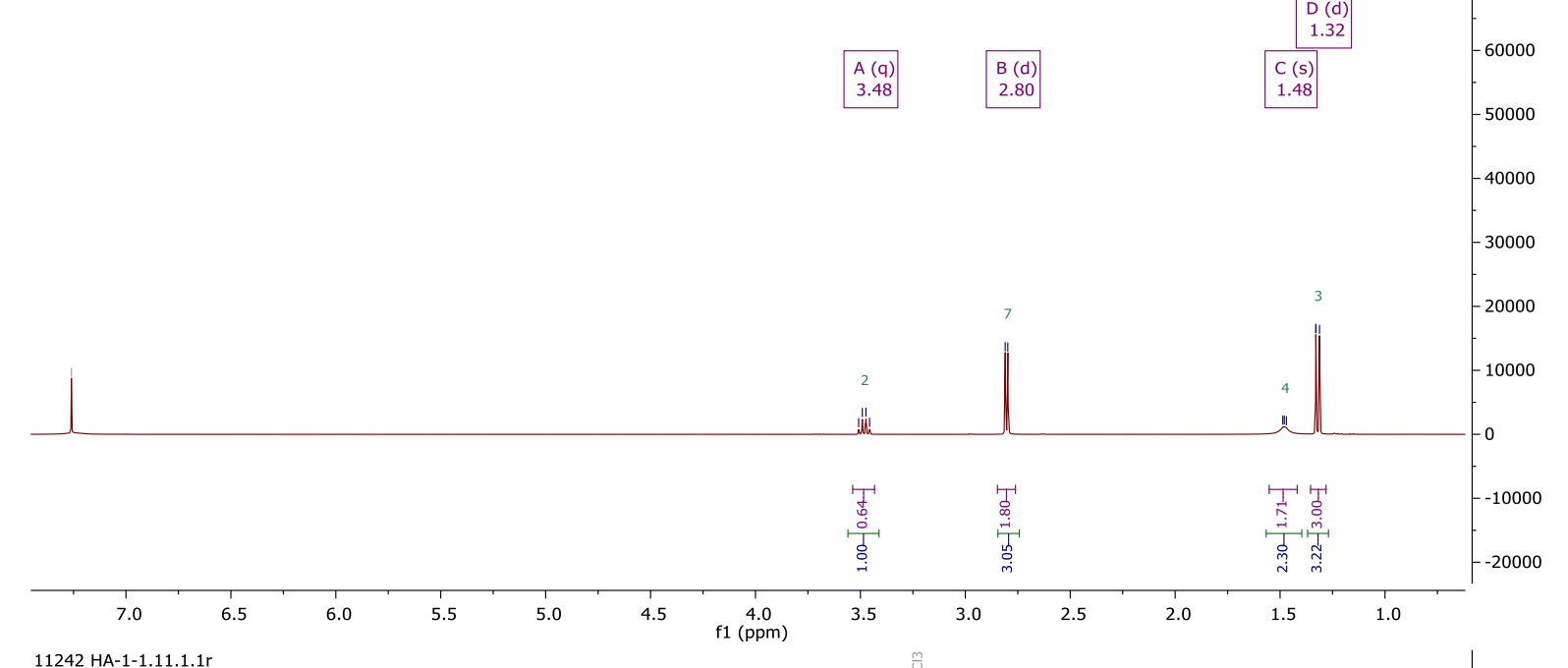

\begin{tabular}{|l|}
\hline $\mathrm{D}(\mathrm{d})$ \\
1.32 \\
\hline $\mathrm{C}(\mathrm{s})$ \\
1.48 \\
\hline
\end{tabular}

$-70000$ 11242 HA-1-1.11.1.1r กิ

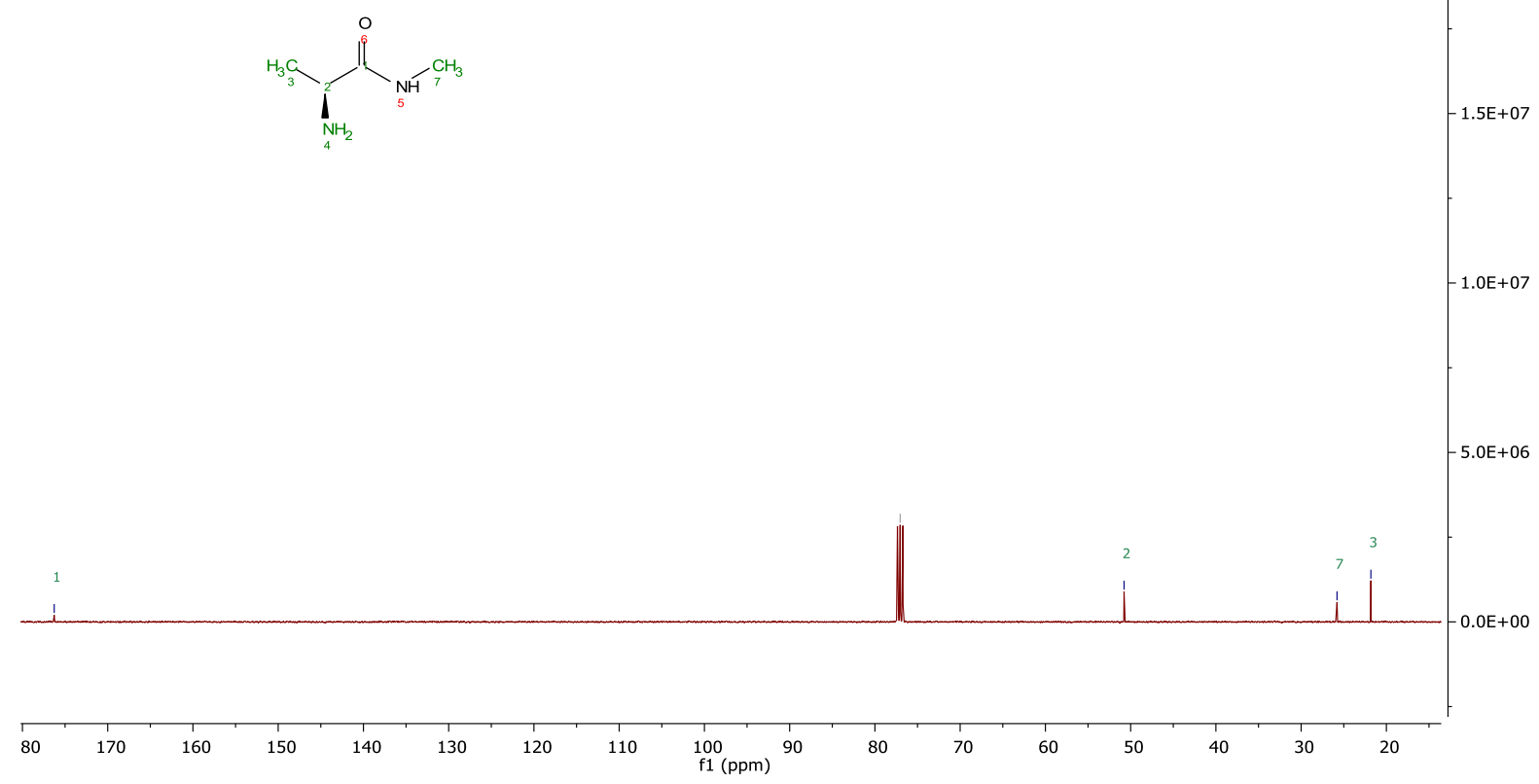


S2

jc46242_JC-03_PROTON_01

in<smiles>CNC(=O)[C@H](C)/N=C/C(C)(C)C</smiles>

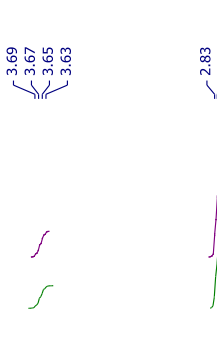

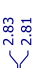

-3200

$\int_{5}^{2}$

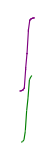

$-2800$

\begin{tabular}{|l|l|}
\hline$A(s)$ & $\begin{array}{l}B(s) \\
77.50\end{array}$ \\
6.90 \\
\hline
\end{tabular}

\begin{tabular}{|l|l|}
\hline $\mathrm{C}(\mathrm{q}$ & $\mathrm{D}(\mathrm{d}$ \\
3.66 & 2.82 \\
\hline
\end{tabular}

E(d)

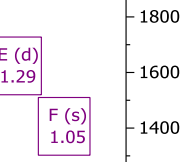

$4,5,6 \quad-1200$

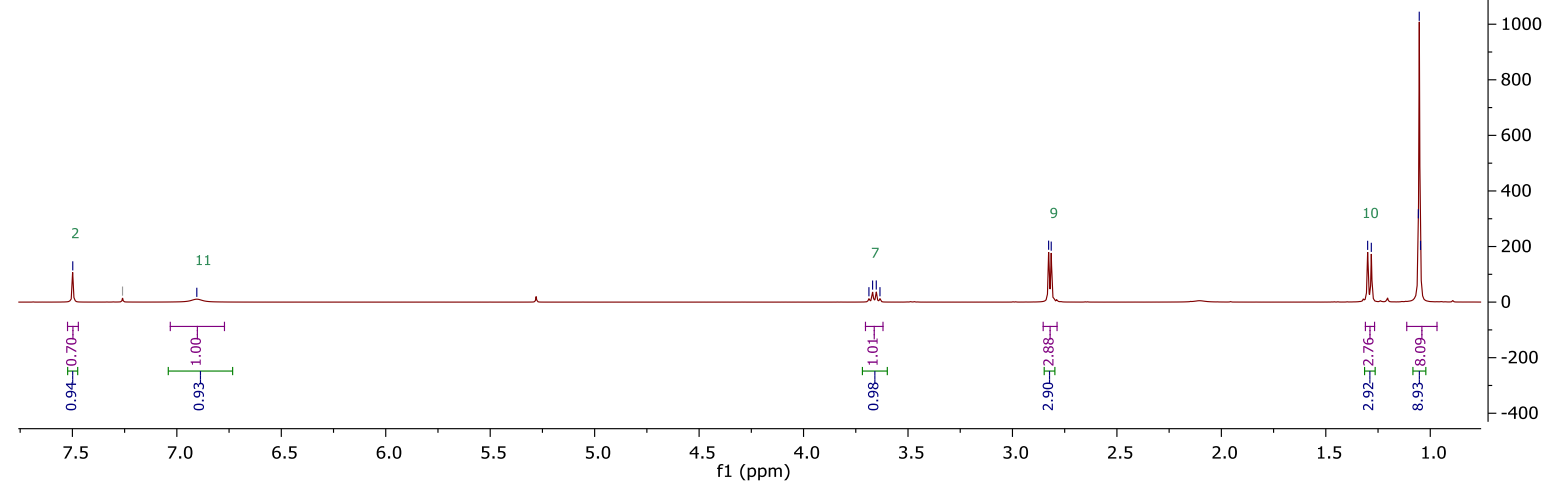

9450 JC-03.11.fid

年
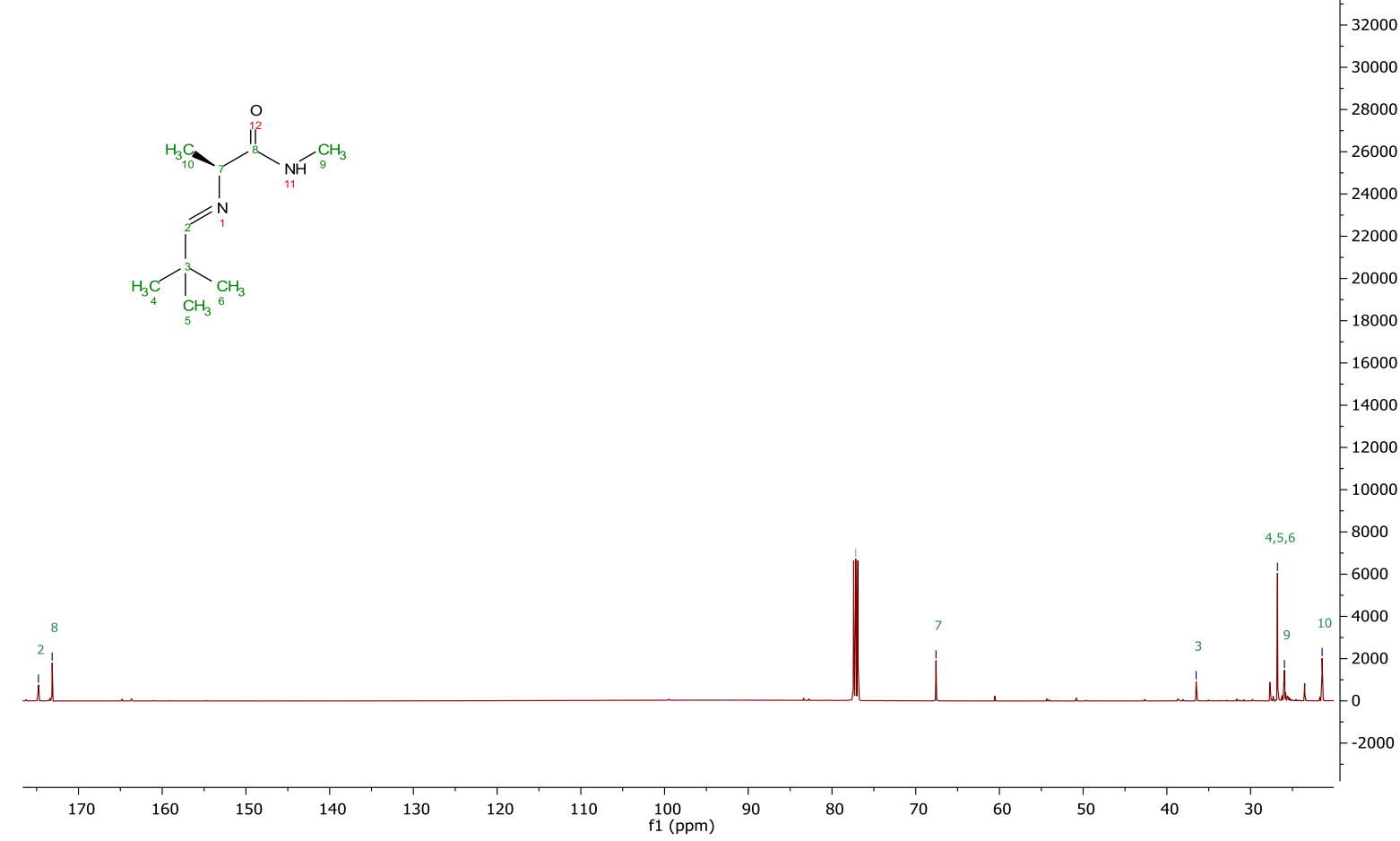
1a

ha37539_HA-31-AlaCC-Trit-SOL_PROTON_01<smiles>CC(C)(C)[Ge]</smiles><smiles>C[C@@H]1C(=O)N(C)[C@H](C(C)(C)C)N1C(=O)Cl</smiles>
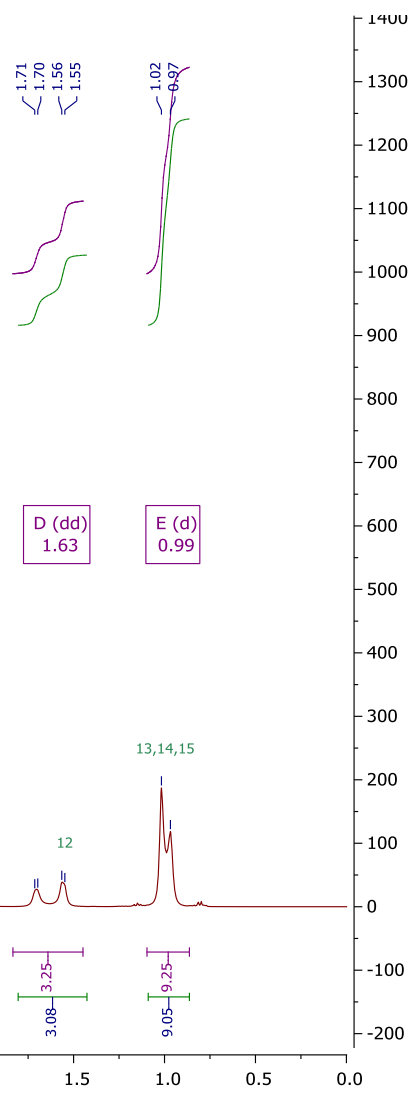

9467 JC-03B recryst.11.fid

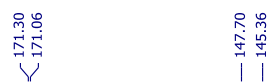
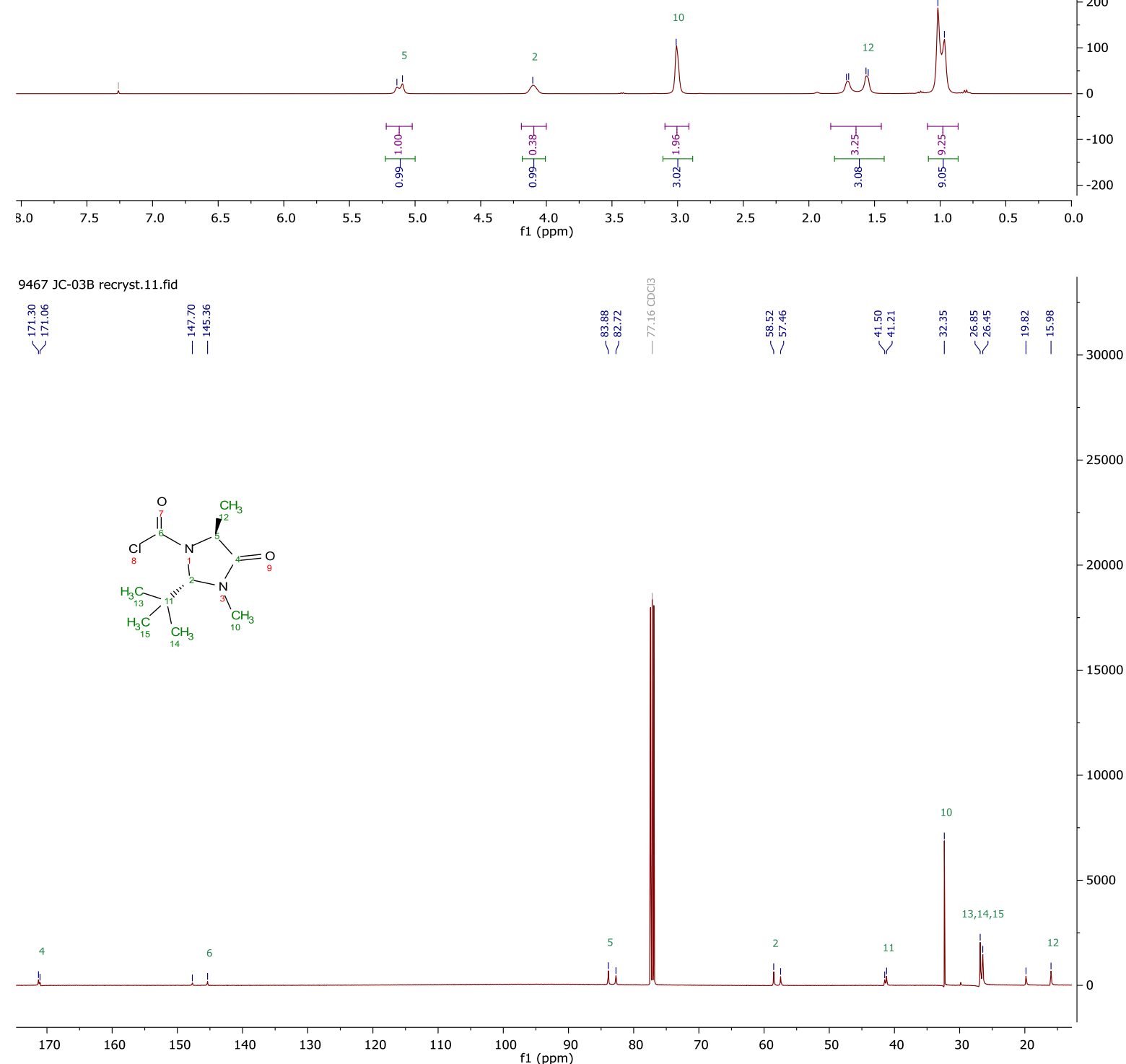
2a

41025 JC-45 full.10.fid

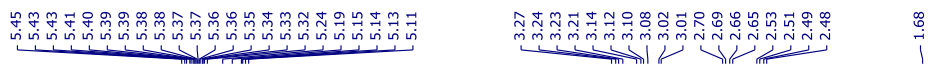

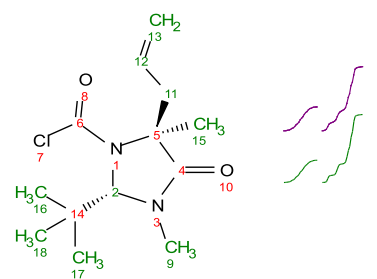

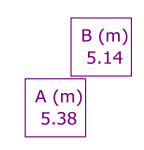
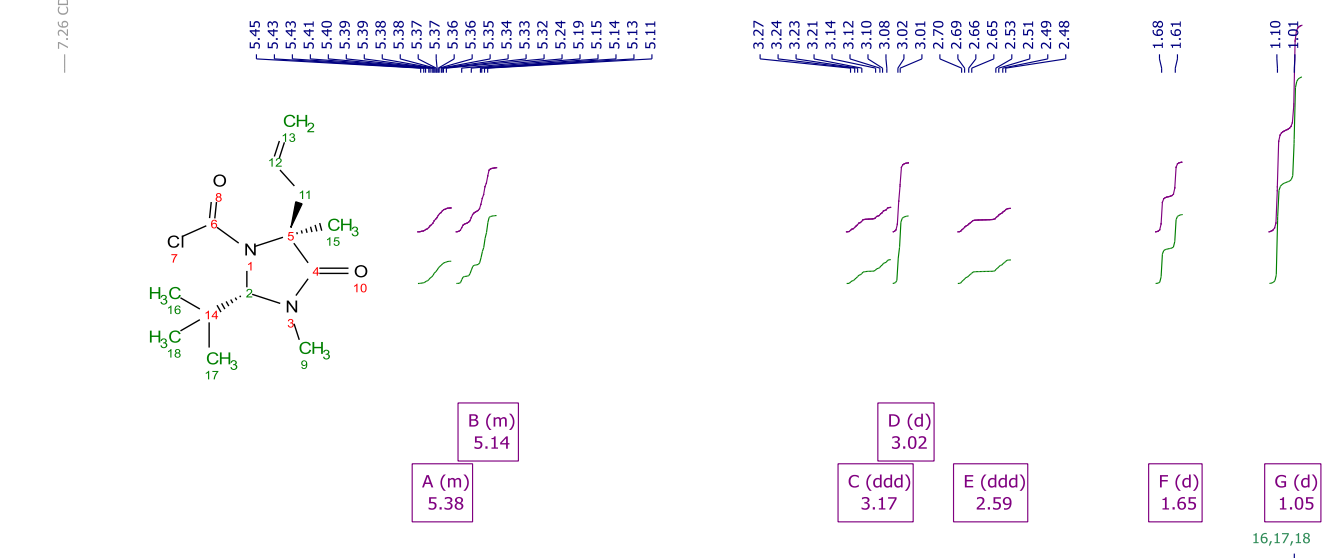

$-10000$

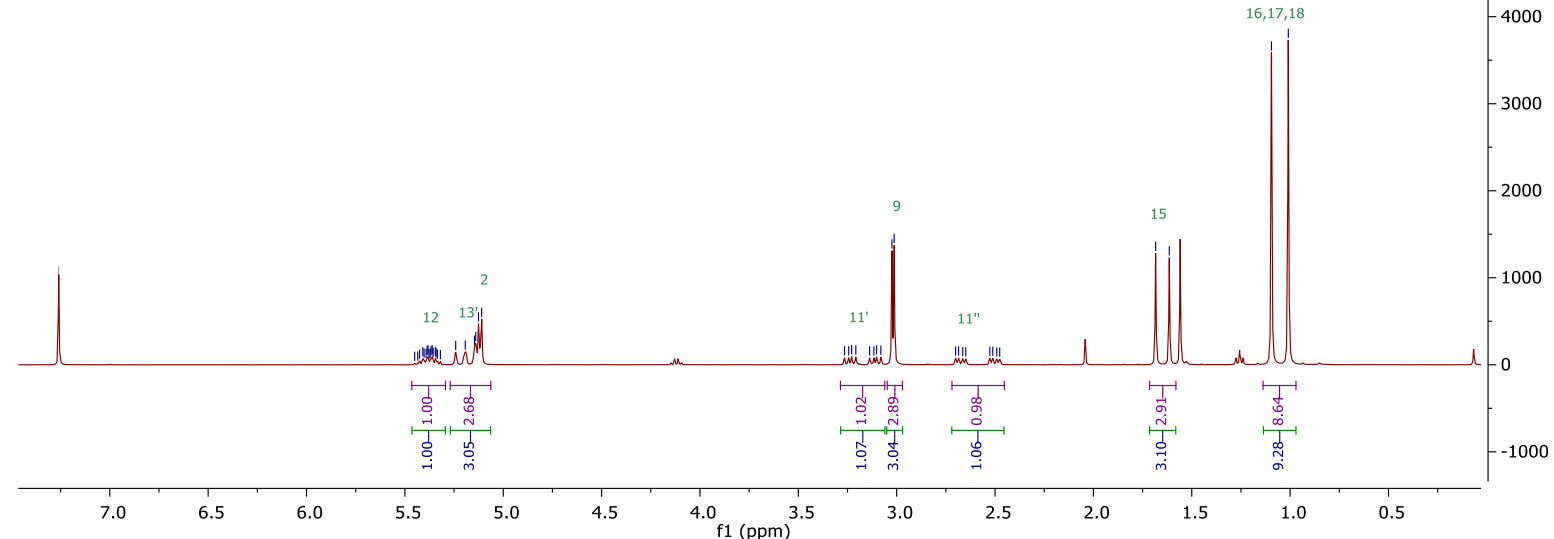

41025 JC-45 full.14.fid

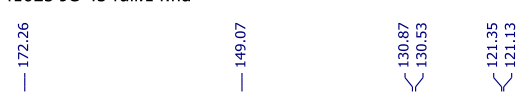
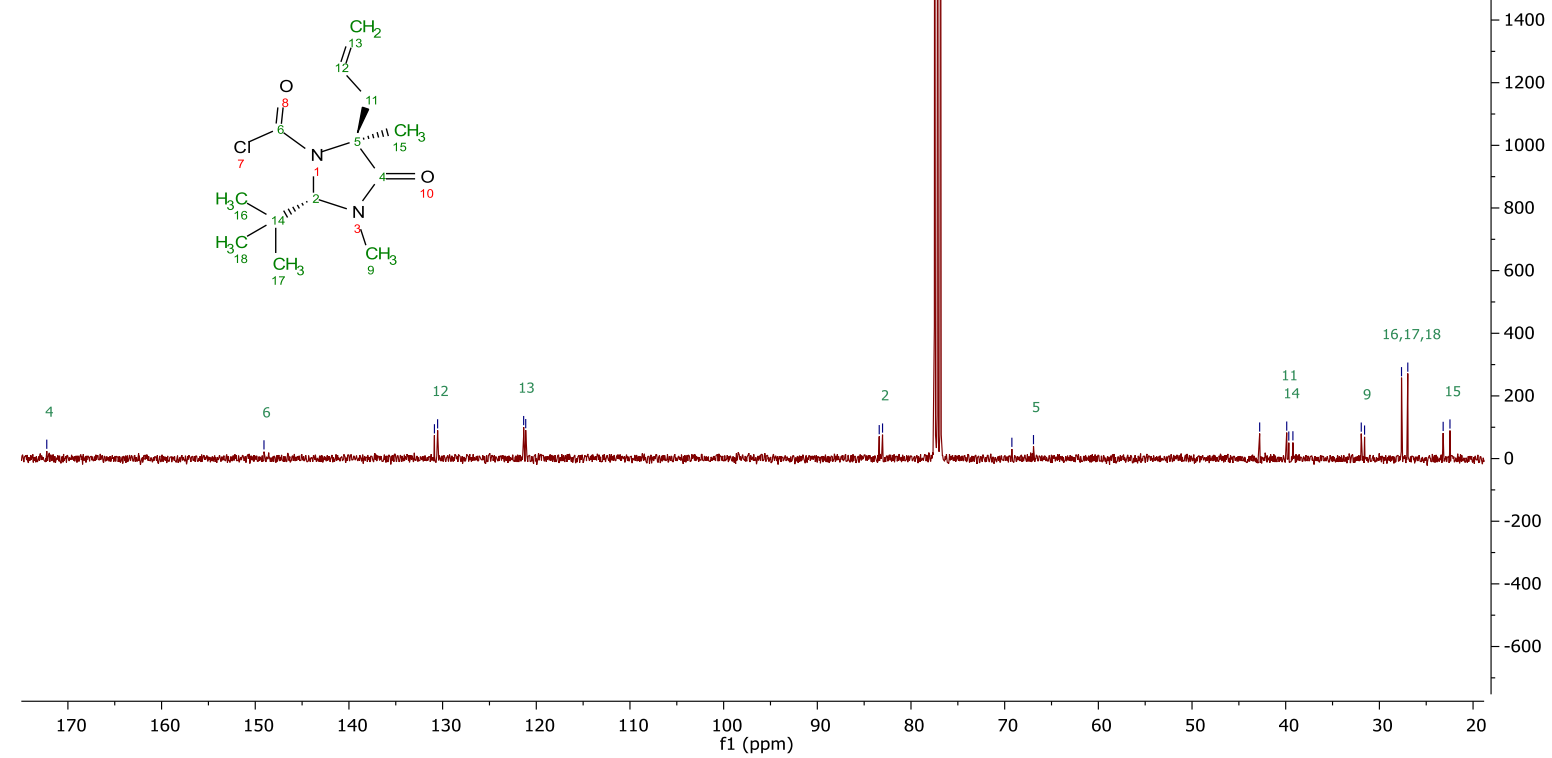
$2 b$

jc48896_JC17_prôduct_after_chrom_PROTON_01

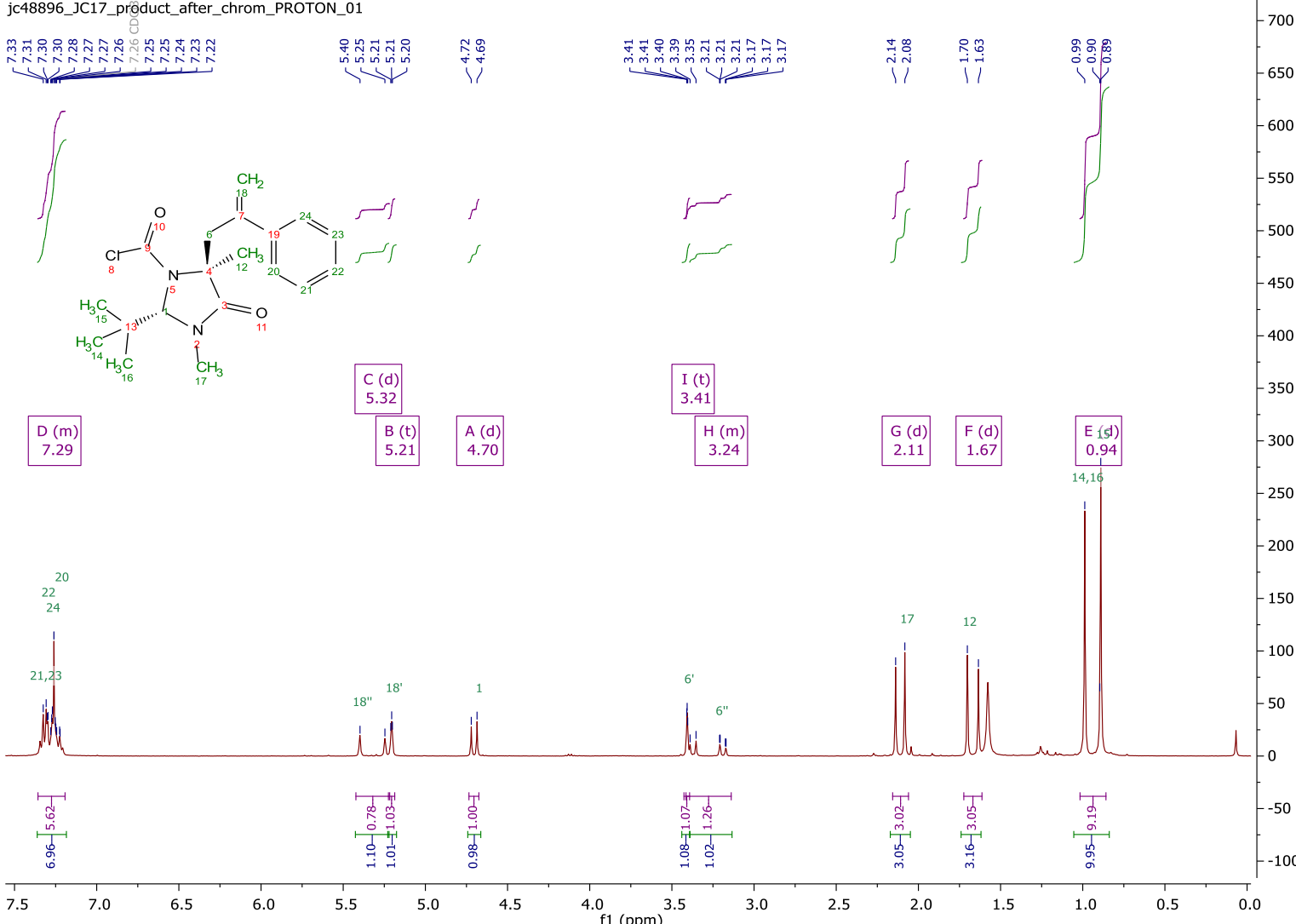

2b

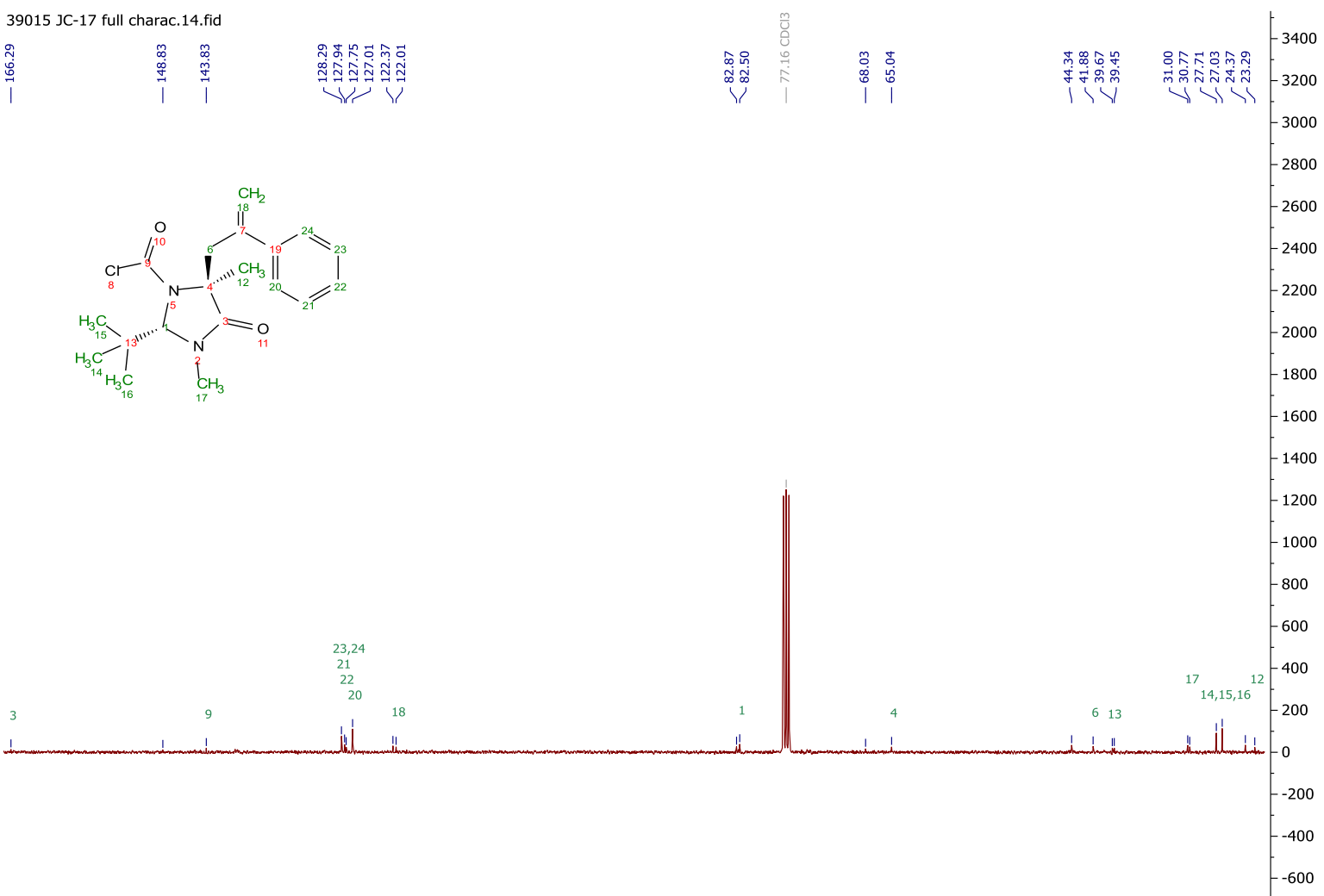

$\begin{array}{lllllllllllllllllllllllllllllllllllllllllllll}165 & 160 & 155 & 150 & 145 & 140 & 135 & 130 & 125 & 120 & 115 & 110 & 105 & 100 & 95 & 90 & 85 & 80 & 75 & 70 & 65 & 60 & 55 & 50 & 45 & 40 & 35 & 30 & 25\end{array}$ 
2c

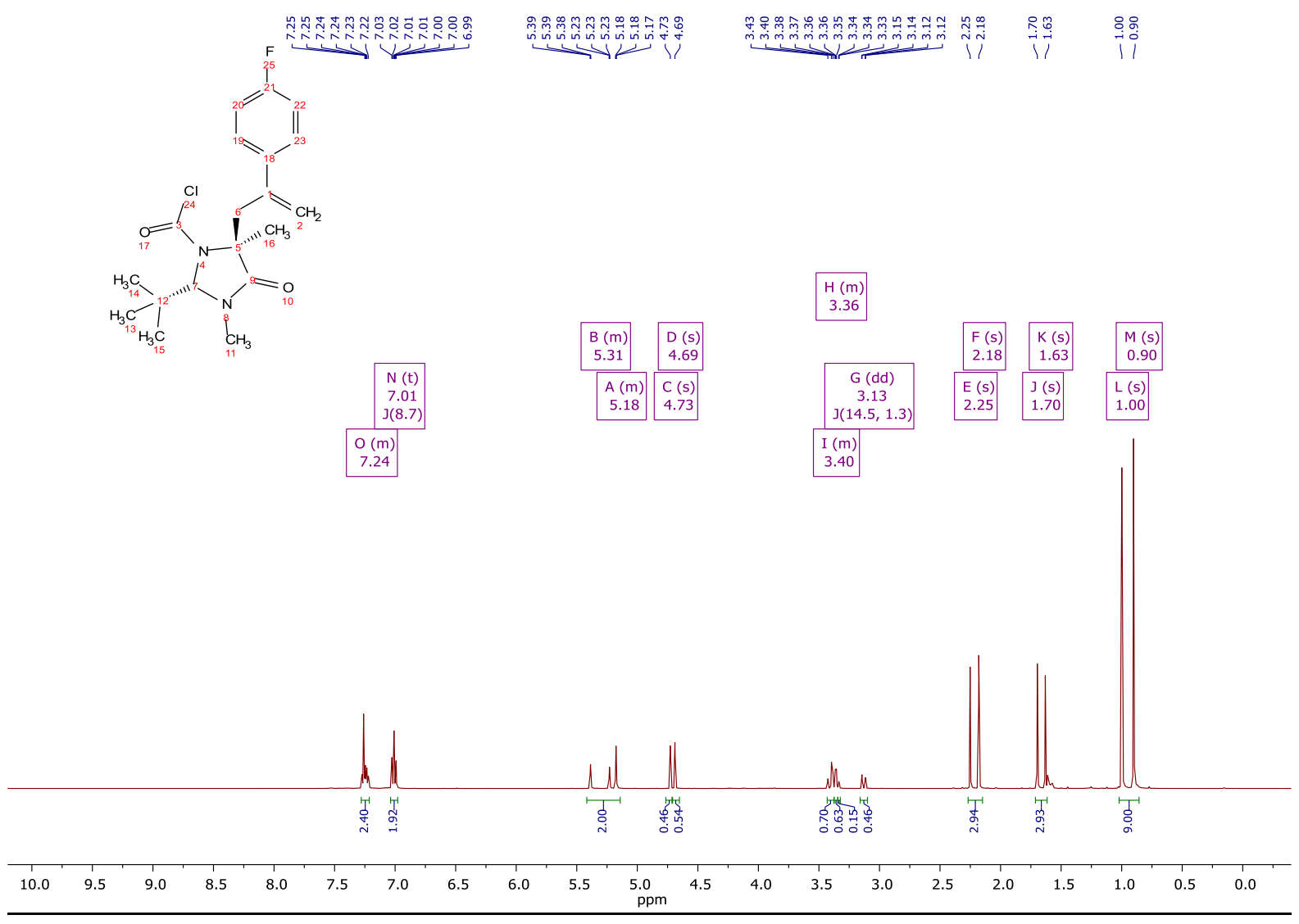

2c

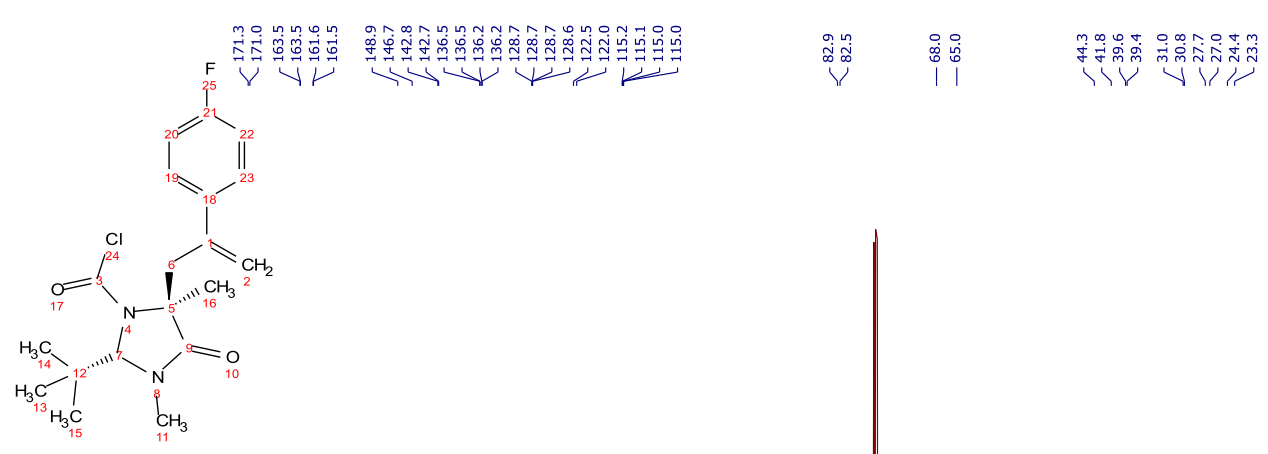

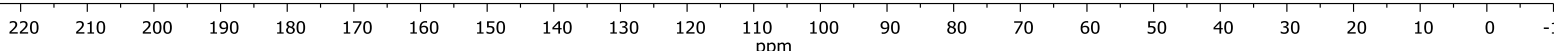


2d
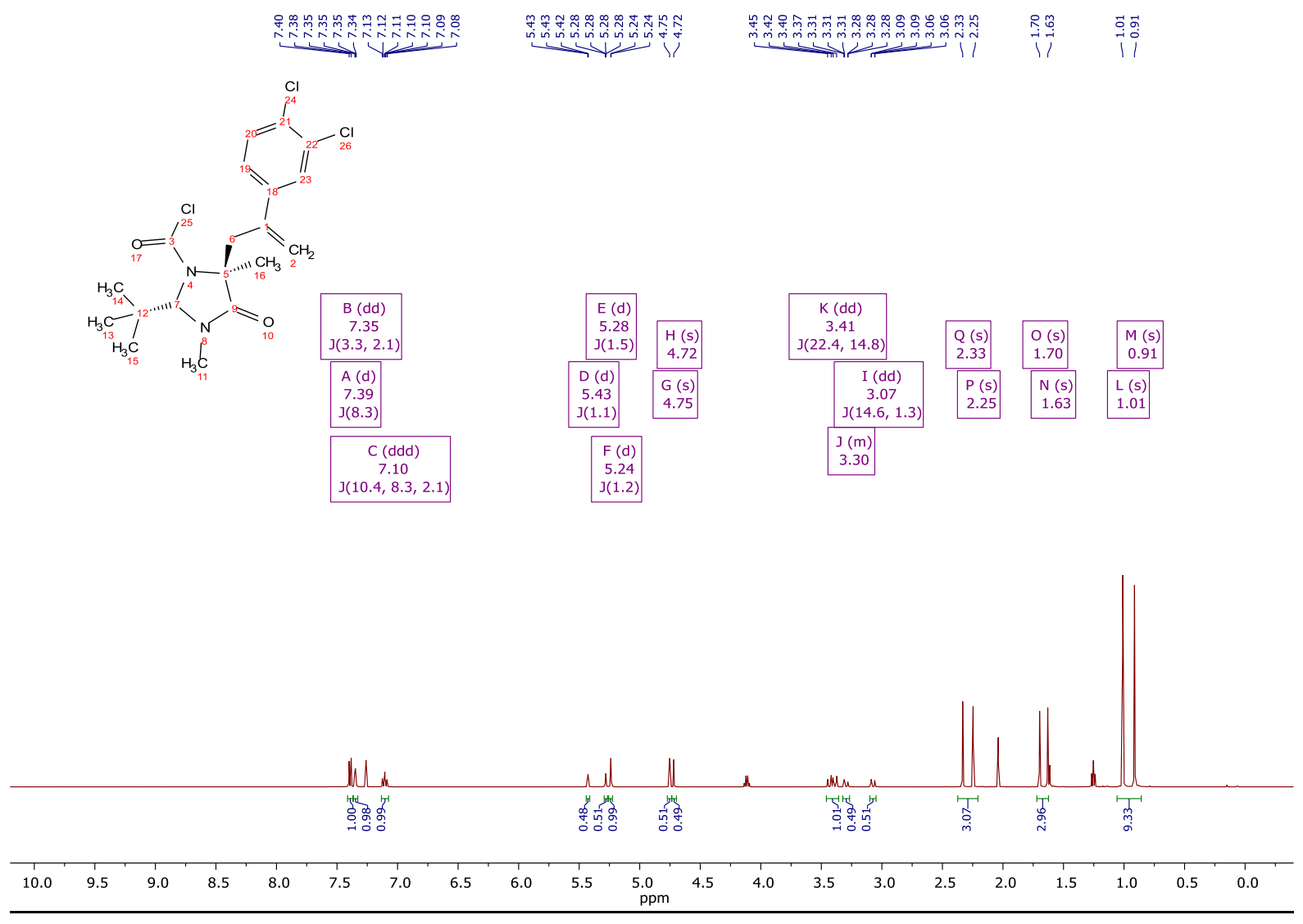

2d

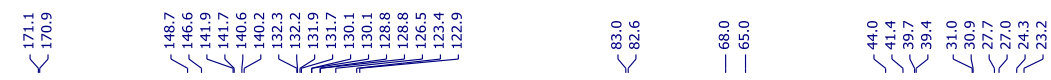
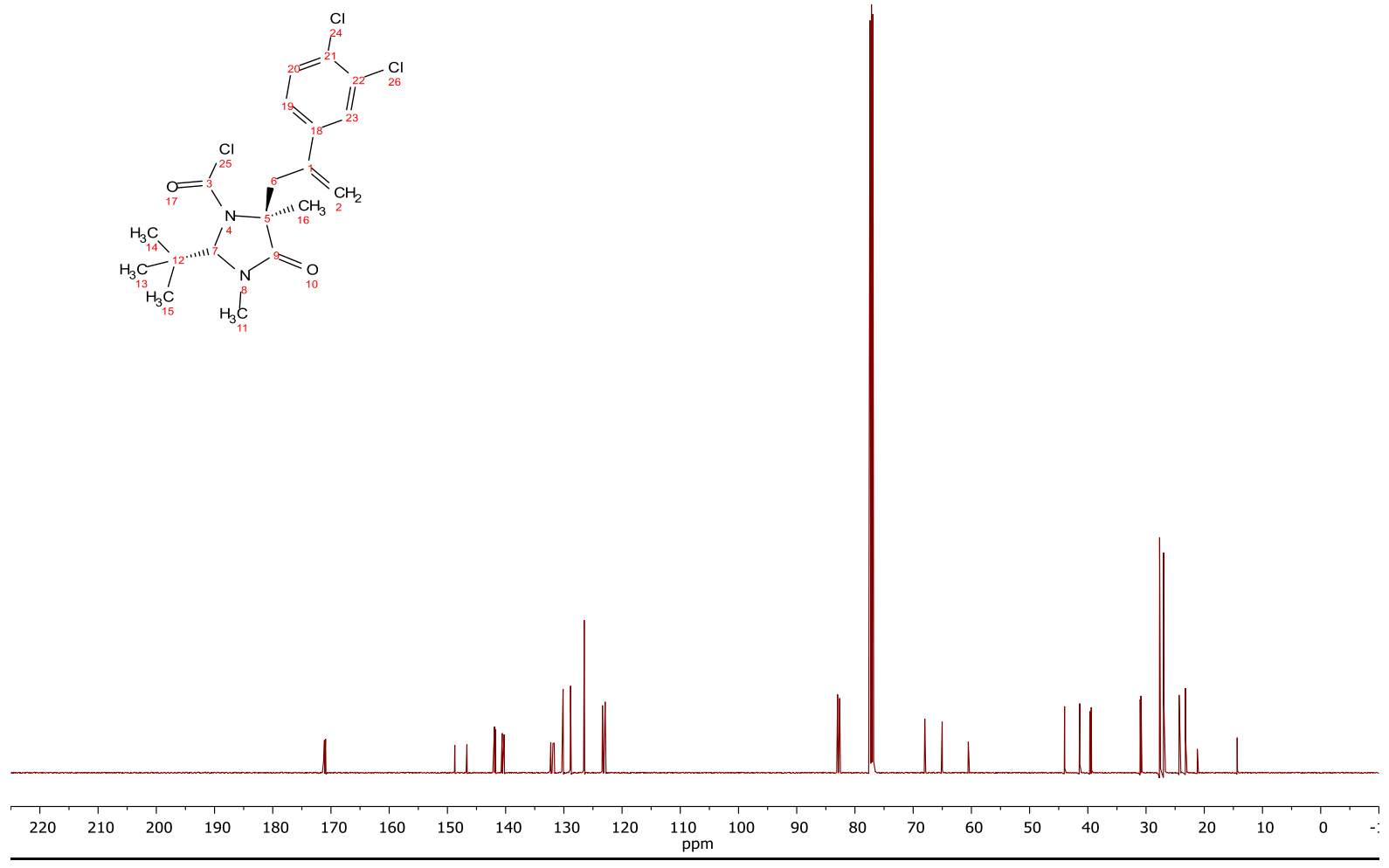

| $|1| \|$

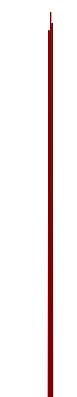


$2 \mathrm{~g}$

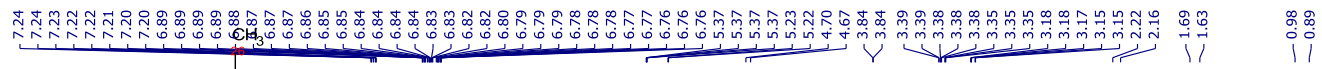

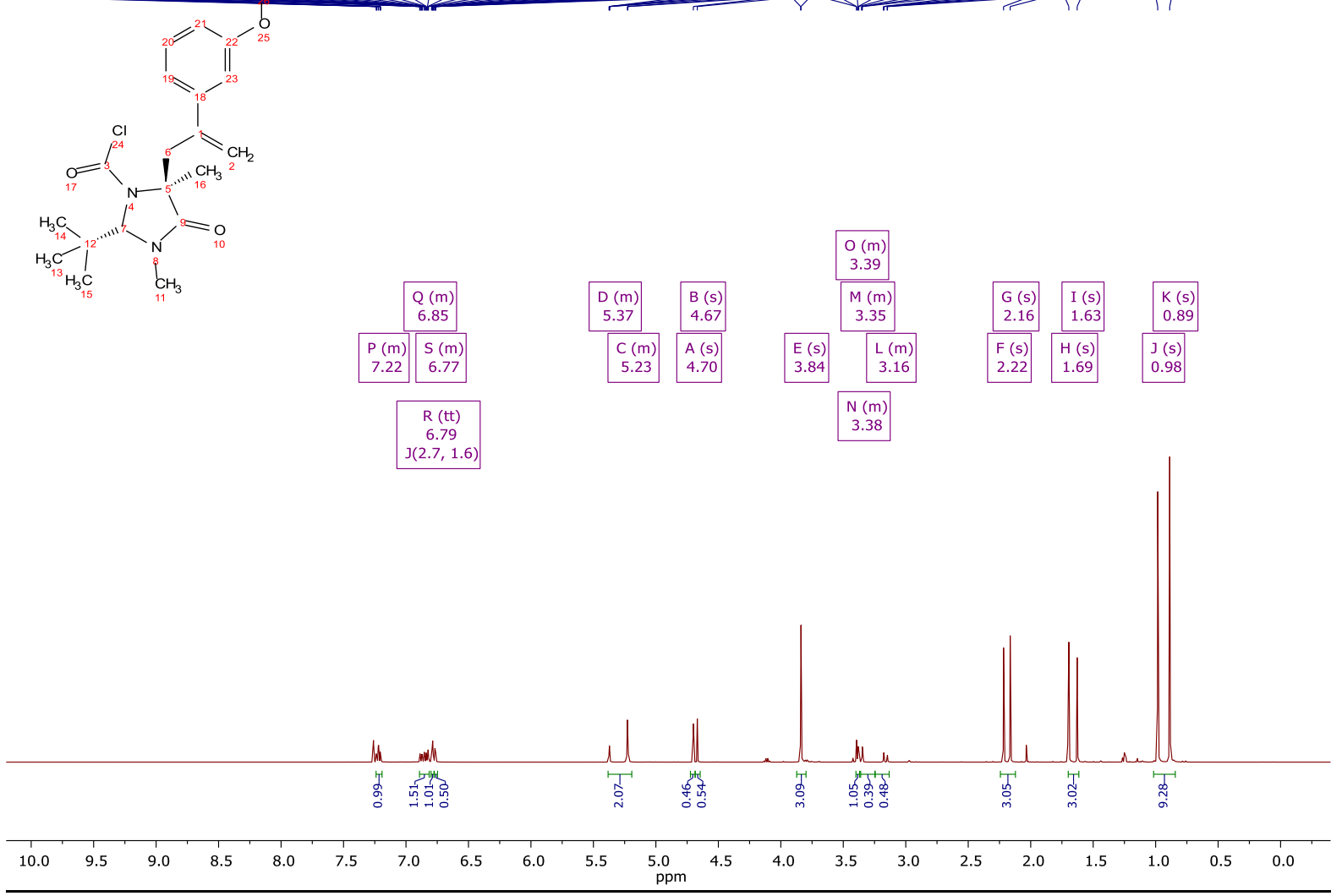

$2 \mathrm{~g}$

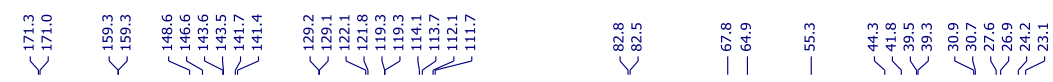

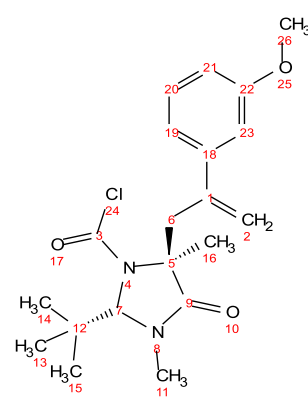

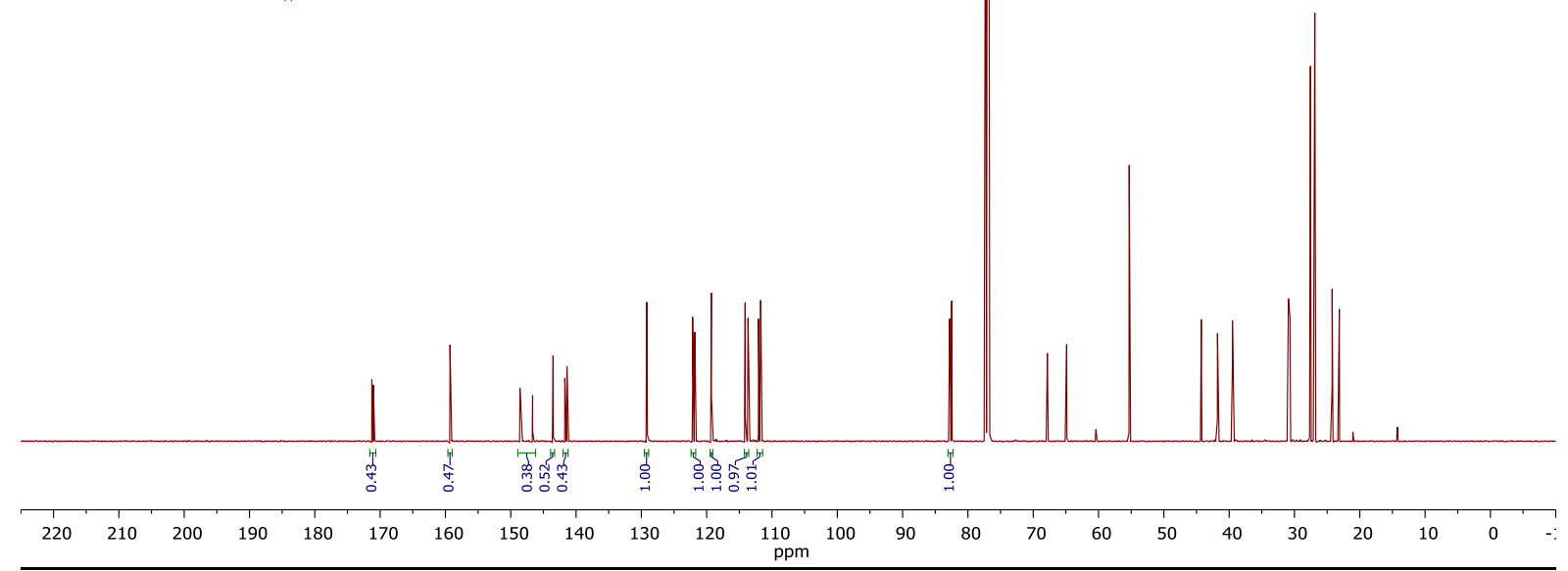


$2 e$

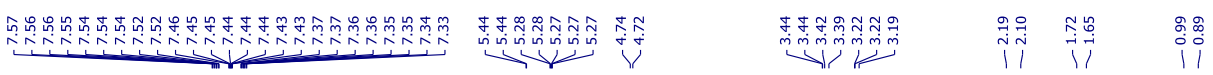

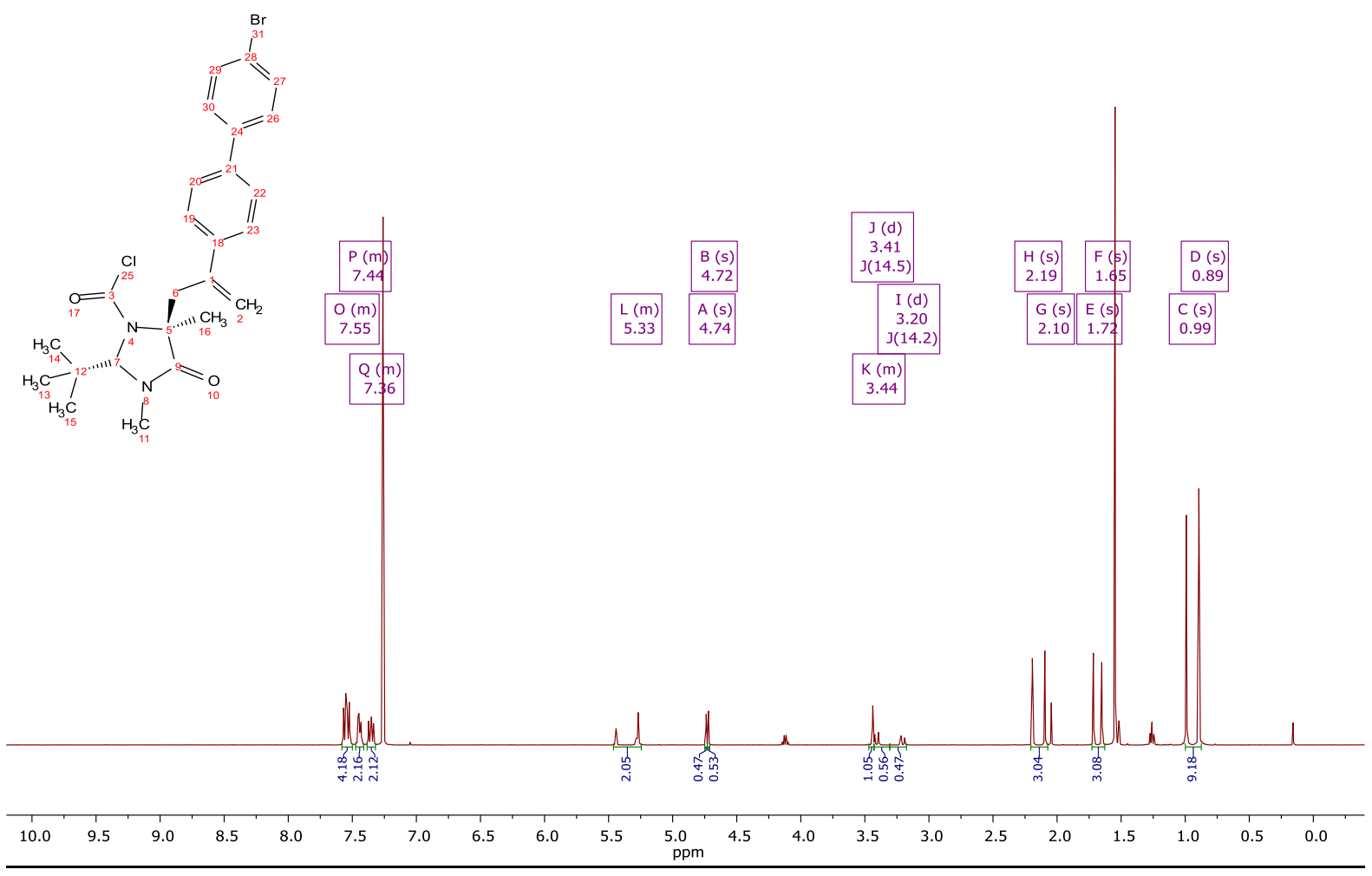

$2 e$

Y

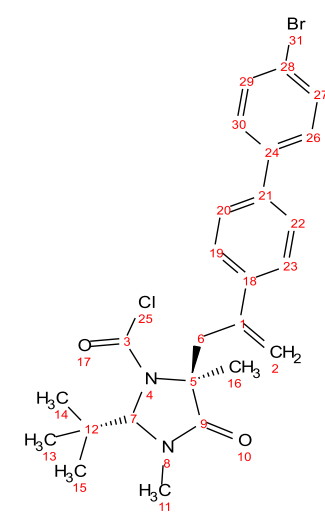

111

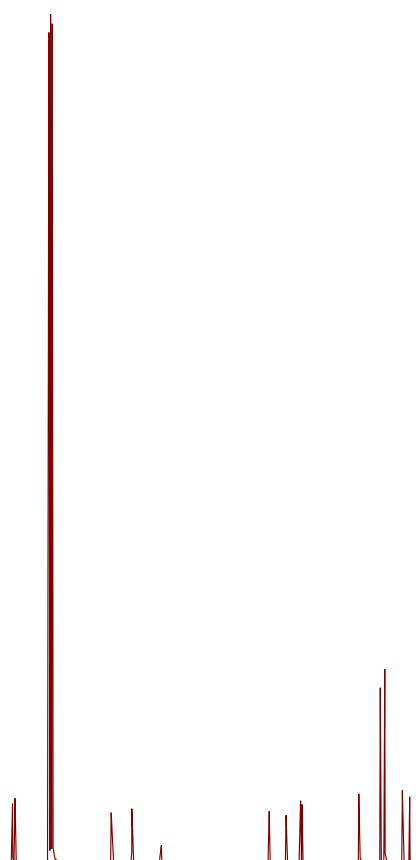

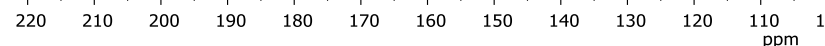


2k

39018 JC-24 full charac.10.fid

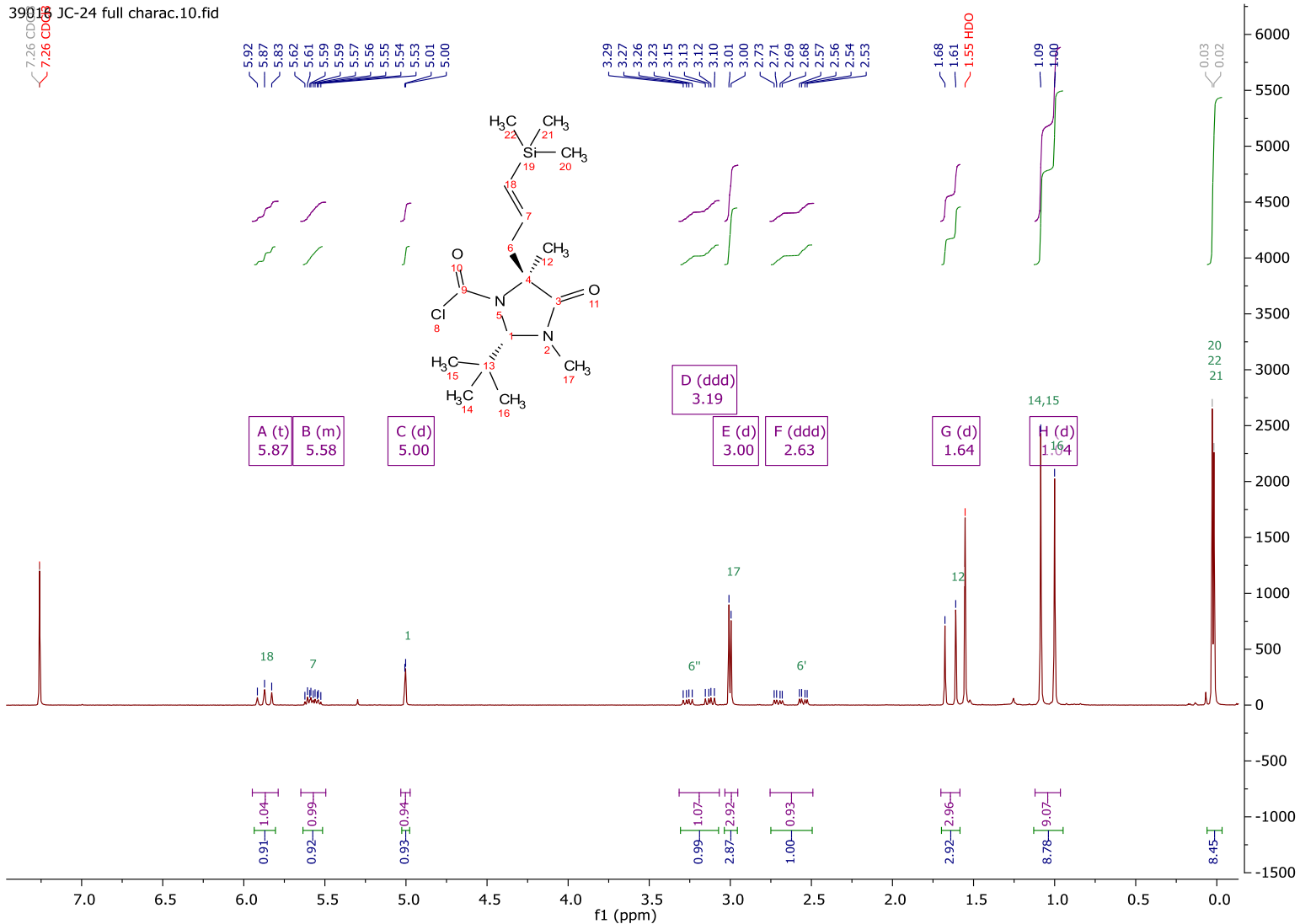

39016 JC-24 full charac.14.fid

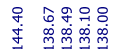

1 r
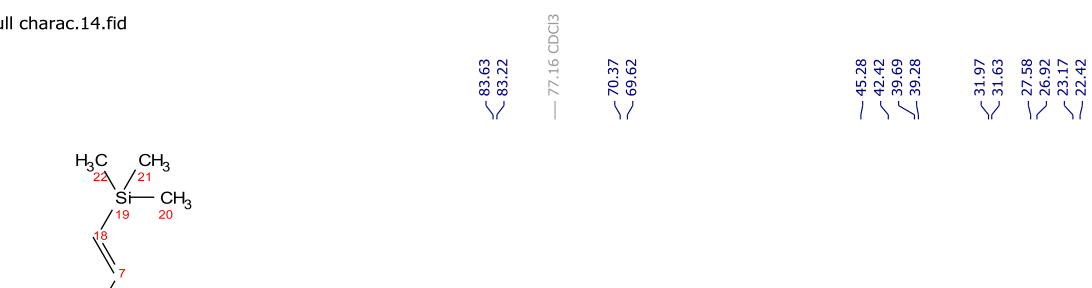

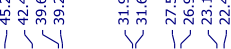

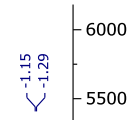

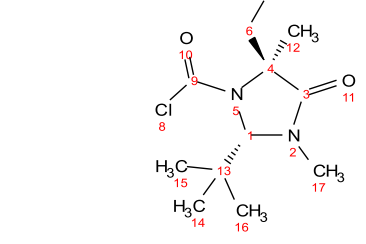


2f

9816 JC-38.10.fid

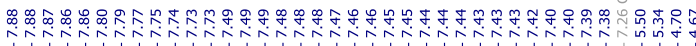

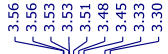

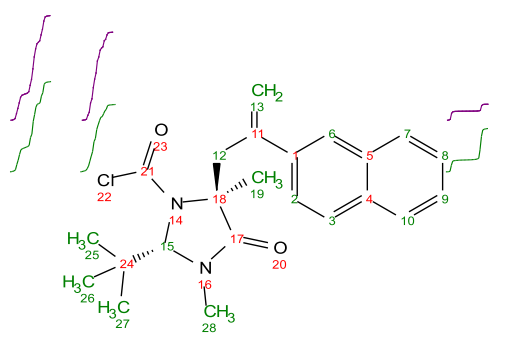

\begin{tabular}{|c|c|}
\hline$A(m)$ & $B(m)$ \\
7.79 & 7.44 \\
\hline
\end{tabular}

C (d) D (d)

\begin{tabular}{ll}
$\mathrm{C}$ & $\mathrm{d}$ \\
\hline
\end{tabular}

r

$\iint$

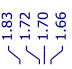

$-60000$

55000

50000

40000

35000

\begin{tabular}{|l|l}
\hline$G(d)$ & -30000 \\
\hline 1.69 & \\
\hline$F$
\end{tabular}

$E(m)$

\begin{tabular}{l}
$E(m)$ \\
3.45 \\
\hline
\end{tabular}

\begin{tabular}{l}
$F(d)$ \\
1.76 \\
\hline
\end{tabular}

0.89

$-25000$

$-20000$

$-15000$

$-10000$

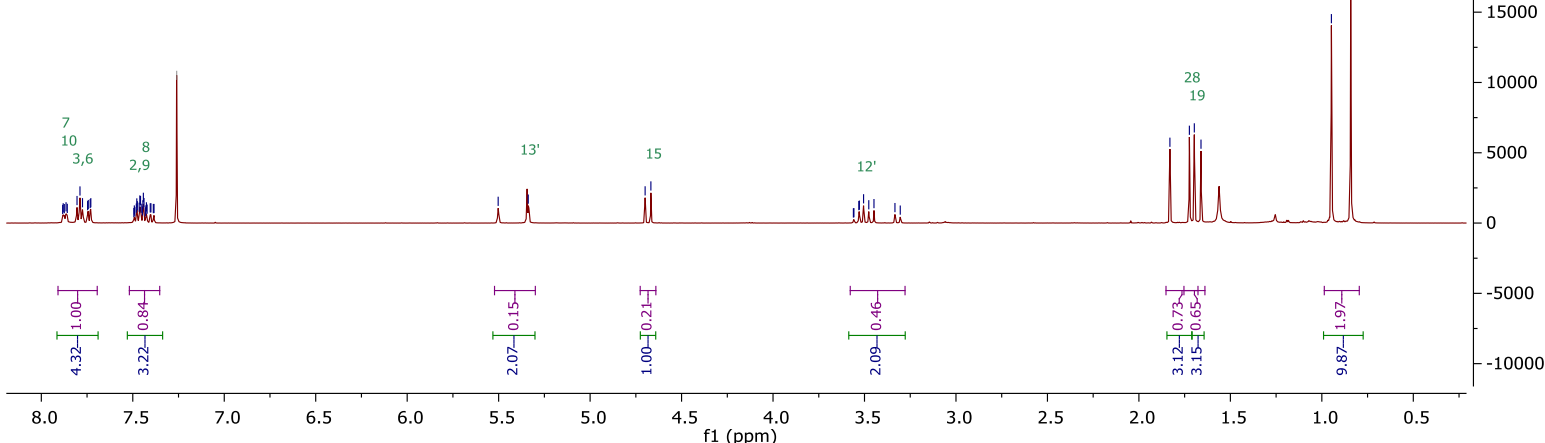

2f

9816 JC-38.11.fid

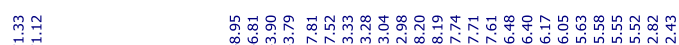

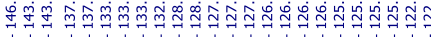

$11 \mathrm{~V}$

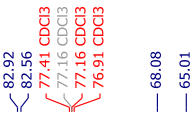

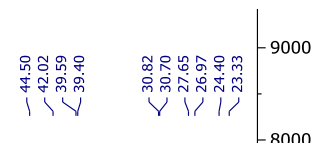

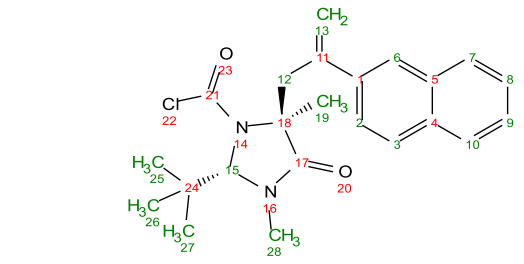

000

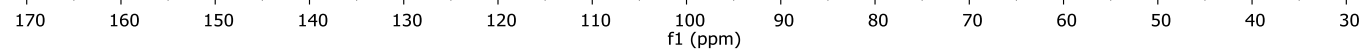


3a

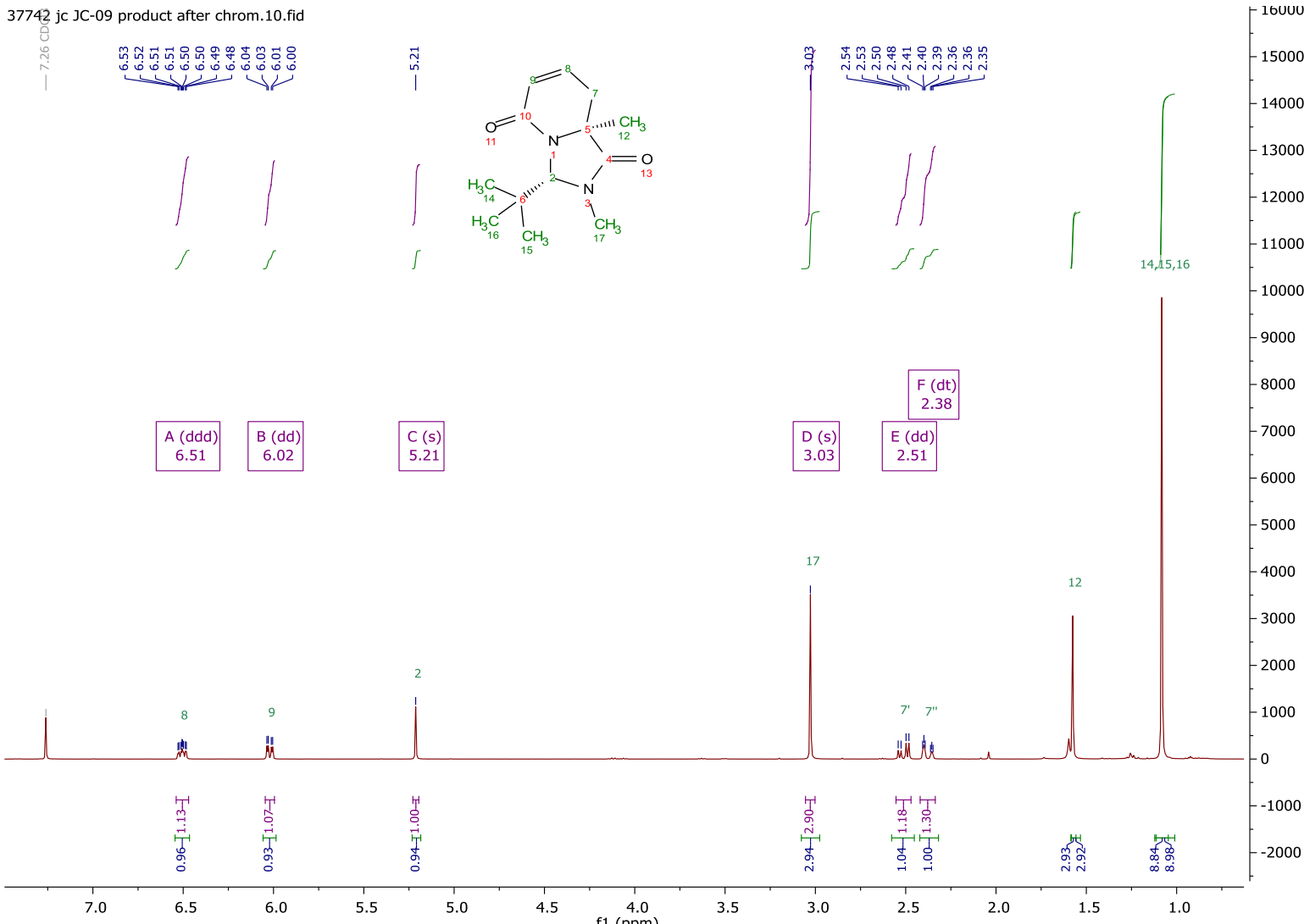

3a

9484 JC-09.11.fic

।<smiles>CC(C)(C)C1N(S)C(=O)P2(=O)CC=CC(=O)N1O2</smiles>

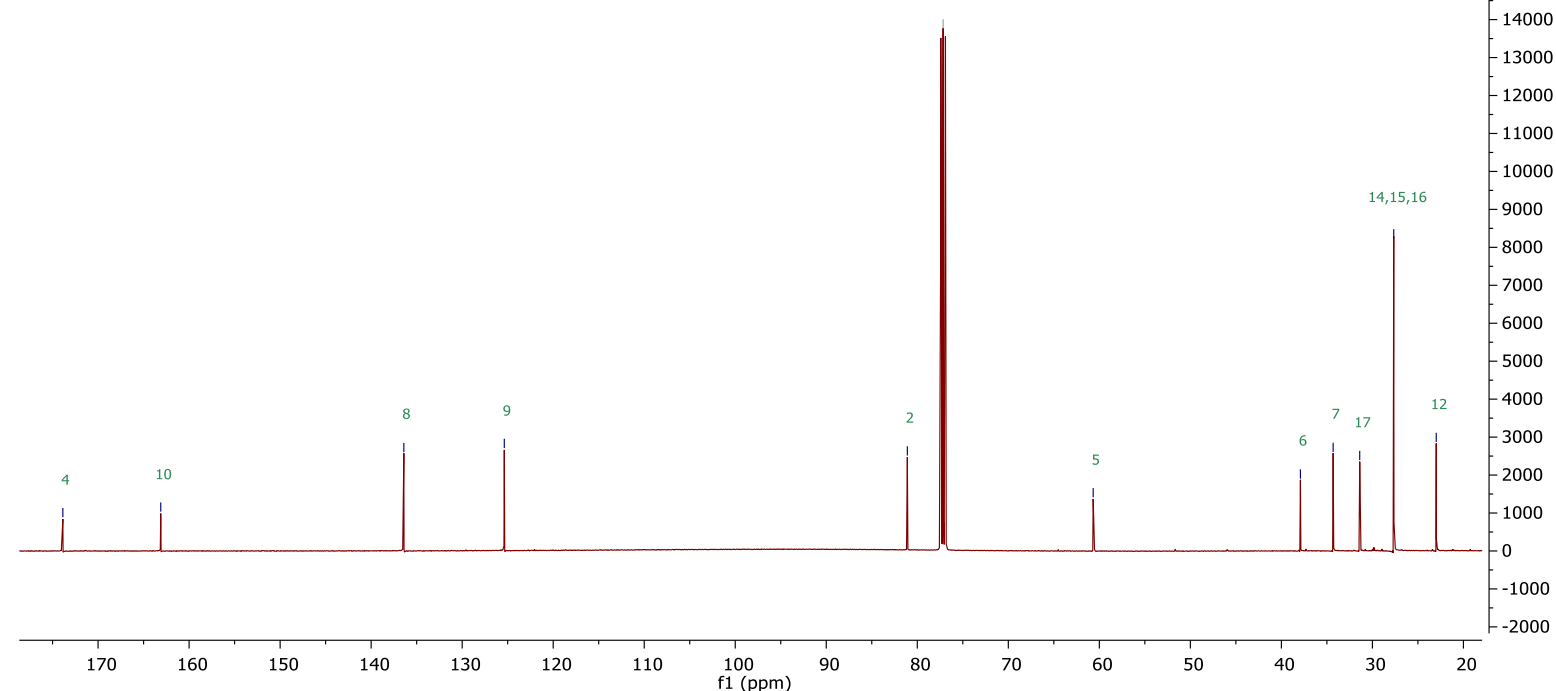


$4 a$

41184 JC- 46 fr 4 full data.10.fid

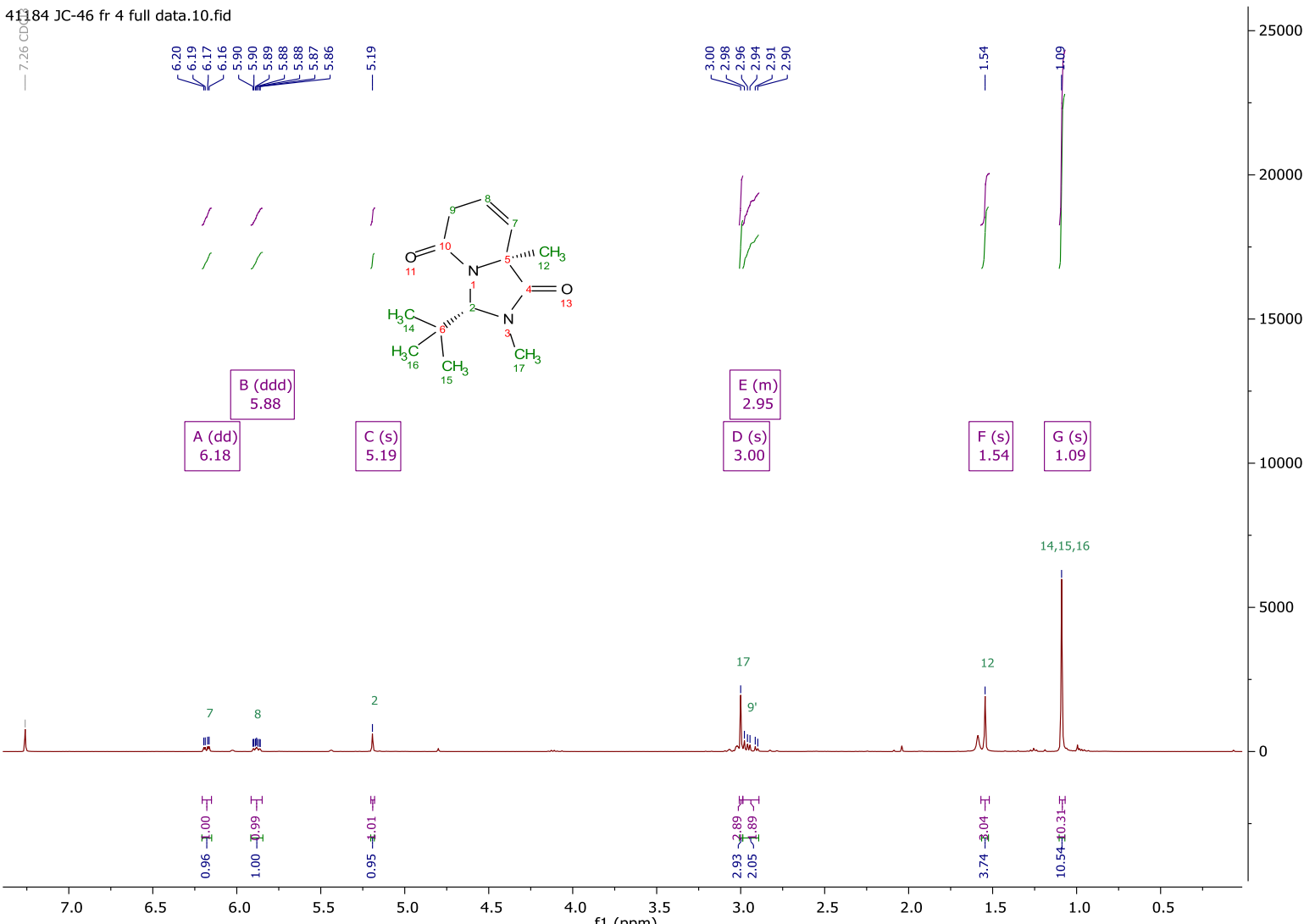

4a

41184 JC- 46 fr 4 full data.14.fid

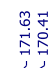
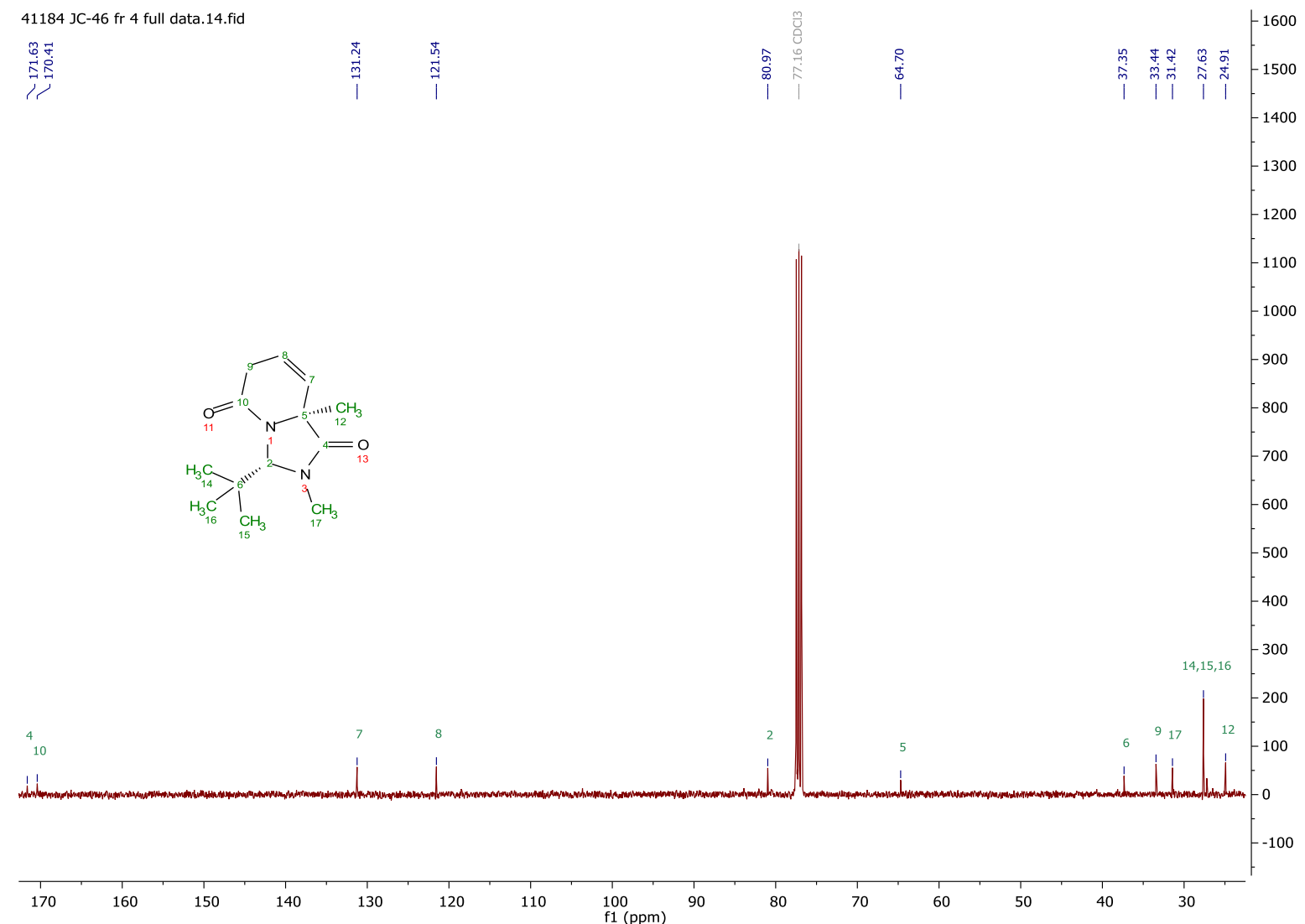
$3 b$

39270 JC-27 P2 fr 8 full charac.10.fid

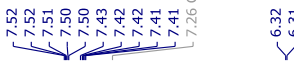<smiles>CCCCC</smiles>
\begin{tabular}{|l|}
\hline$G(d)$ \\
6.32 \\
\hline
\end{tabular}

$F(s)$
5.26

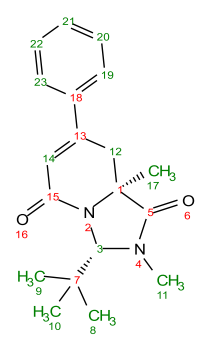

ทำ

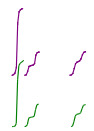

$D .99$
2.99

\begin{tabular}{ll|l}
\hline$E(s)$ & $C(d d)$ \\
3.07 & 2.74 \\
\hline
\end{tabular}

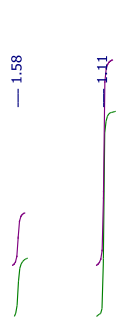

$-16000$

5000

$-14000$

$-13000$

$-12000$

$-11000$

$-10000$

$-9000$

$-8000$

$-7000$

$-6000$
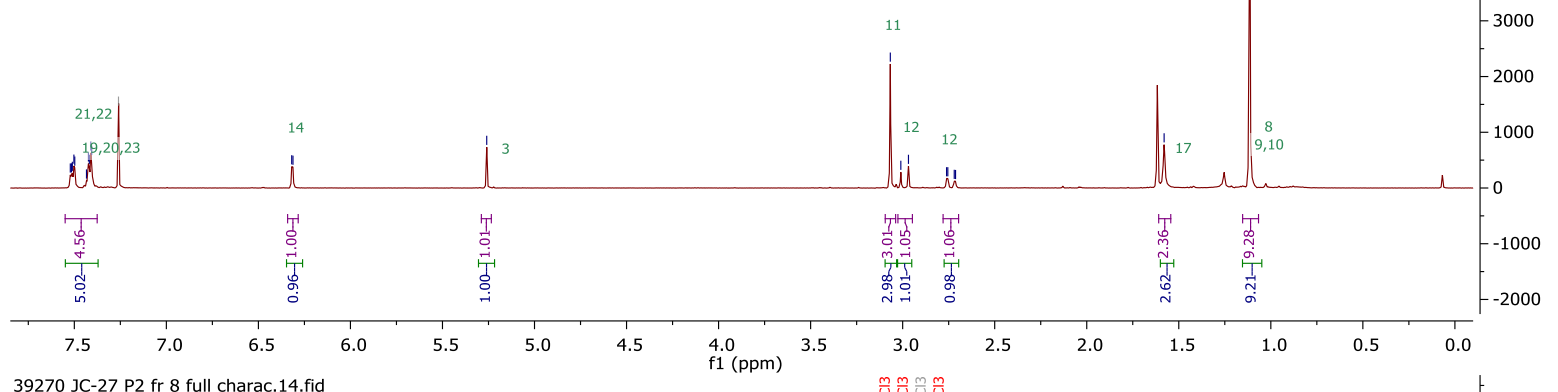
39270 JC-27 P2 fr 8 full charac.14.fid

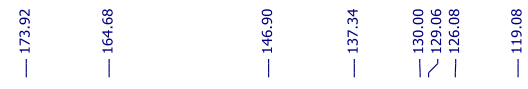

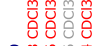

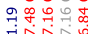<smiles>CC(C)(C)C1N(O)C(=O)C2(C)NC(c3cccnc3)=CC(=O)N12</smiles>

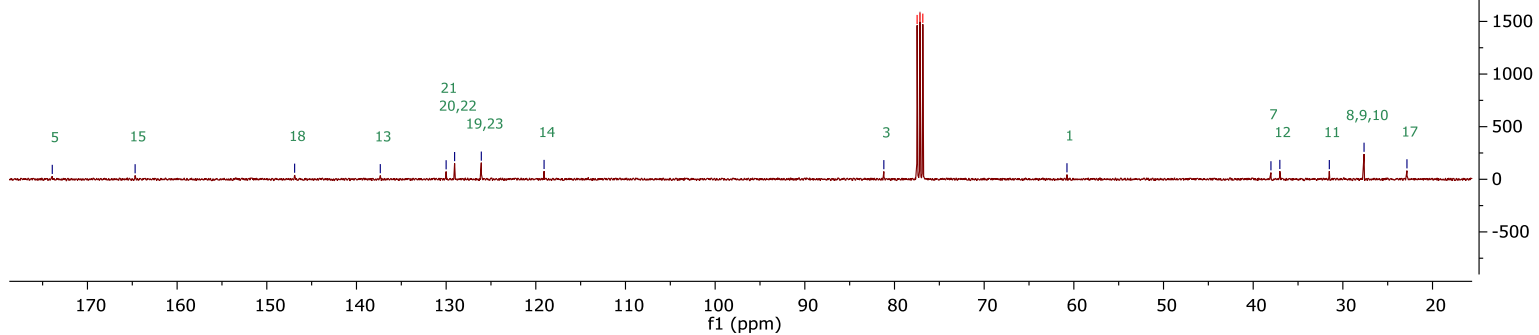


4b

39269 JC-27 P1 fr 7 full charac.10.fid

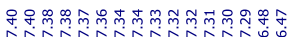

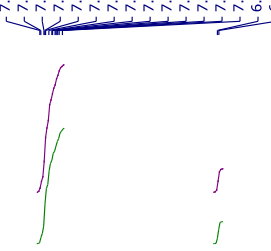

\begin{tabular}{|l|l|}
\hline$A(m)$ & $B(d)$ \\
7.36 & 6.48 \\
\hline
\end{tabular}

48

$\underset{\tilde{n}}{\tilde{n}}$

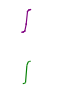

$\left.\prod_{23}^{22}\right|_{18} ^{20}$

C (s)<smiles></smiles>

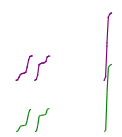

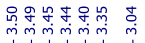

บivim

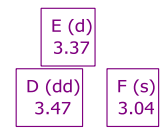

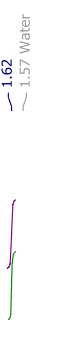

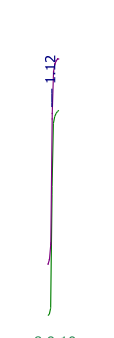

\begin{tabular}{l}
-8500 \\
-8000 \\
-7500 \\
-7000 \\
-6500 \\
\hline-6000 \\
\hline 5500 \\
\hline-5000 \\
\hline-4000 \\
\hline-3500 \\
\hline-3000 \\
\hline-2500 \\
\hline-2000 \\
\hline-1500 \\
\hline-1000 \\
\hline 500 \\
\hline-500 \\
\hline
\end{tabular}

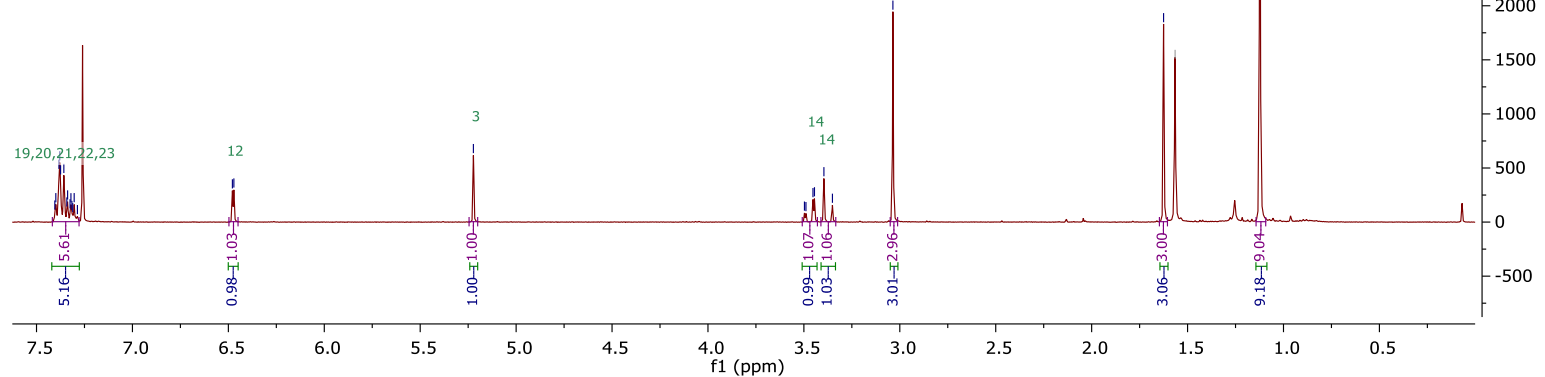

4b

39269 JC-27 P1 fr 7 full charac. 14. fid

ำ

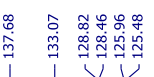

$\begin{array}{lll} & \frac{0}{0} & \\ 0 & 0 & \\ 0 & 0 & 0 \\ 0 & 0 & 0 \\ 0 & 1 & 0 \\ 1 & 1 & 1\end{array}$

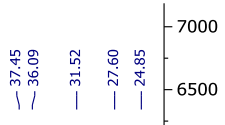<smiles>CN1C(=O)N(O)C(C2(C)CC2)N2C(=O)NC(c3cccnc3)=NC12C</smiles>

6000

5500

000

4500

$-4000$

$-3500$

$-3000$

$-2500$

$-2000$

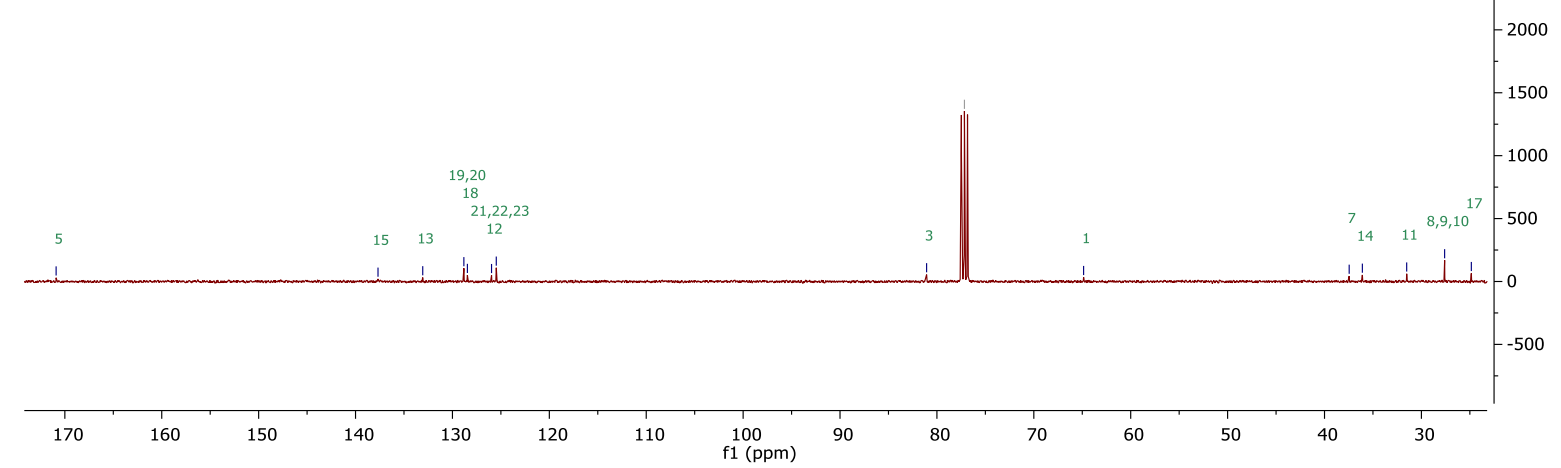


3c

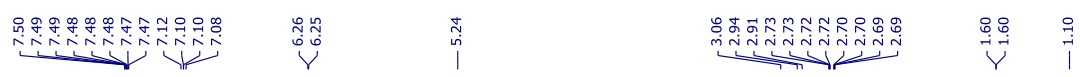

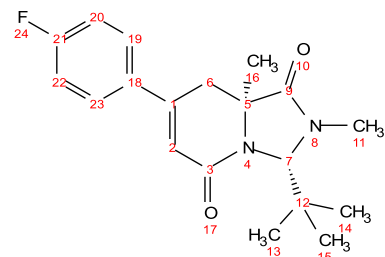

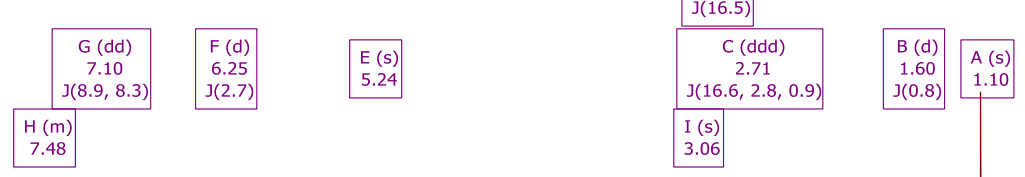

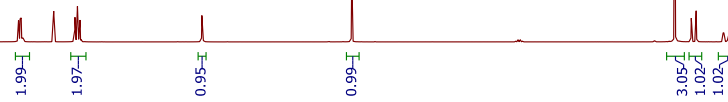

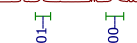

\begin{tabular}{lllllllllllllllllllllll}
\hline 10.0 & 9.5 & 9.0 & 8.5 & 8.0 & 7.5 & 7.0 & 6.5 & 6.0 & 5.5 & $\begin{array}{c}5.0 \\
\mathrm{ppm}\end{array}$ & 4.5 & 4.0 & 3.5 & 3.0 & 2.5 & 2.0 & 1.5 & 1.0 & 0.5 & 0.0 \\
\hline
\end{tabular}

3c

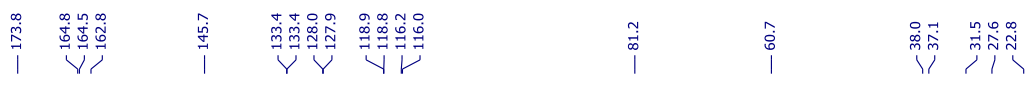

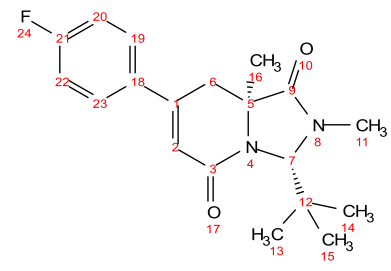

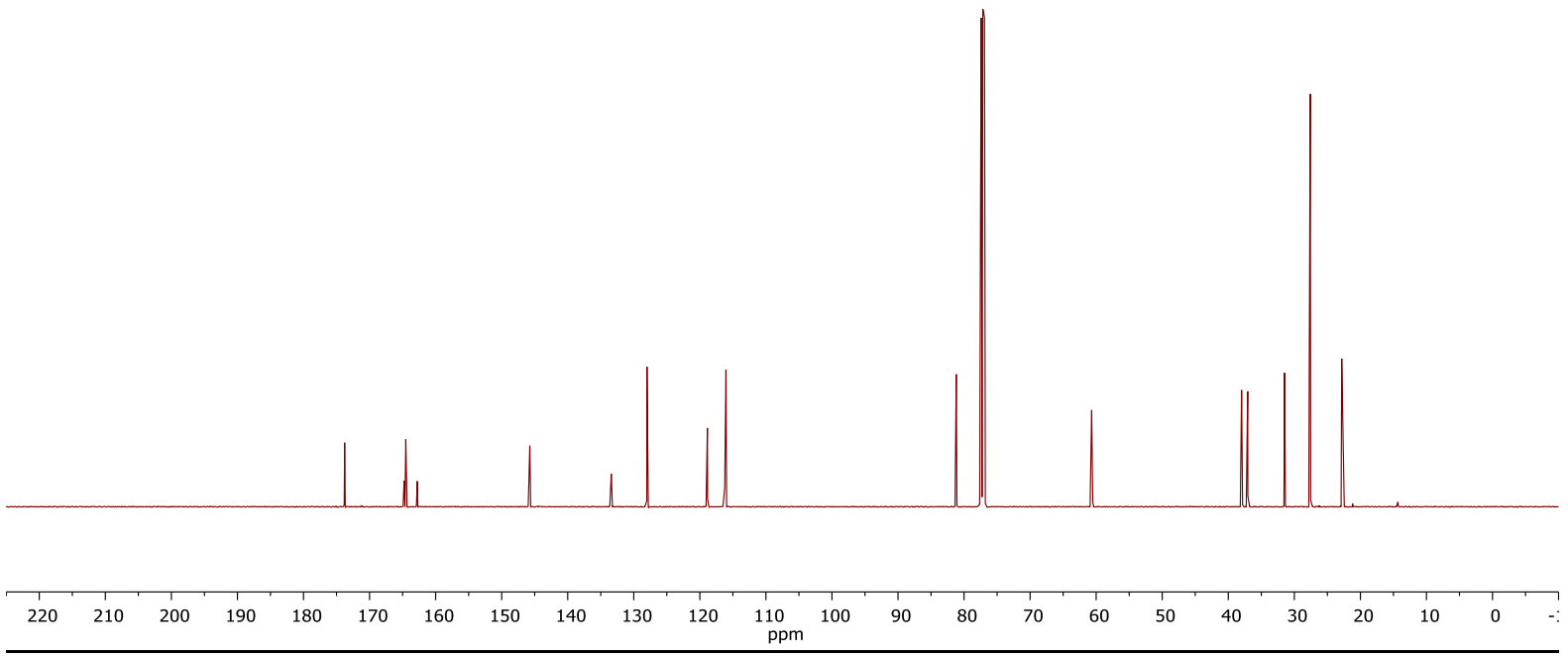


4c

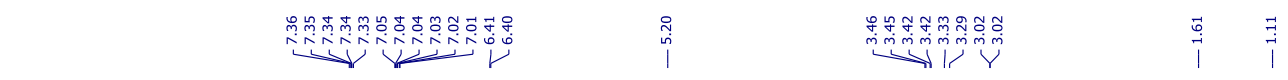

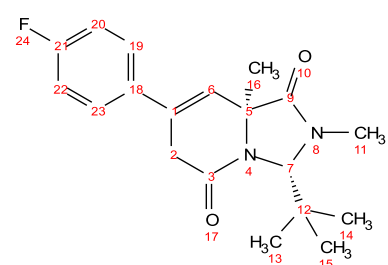

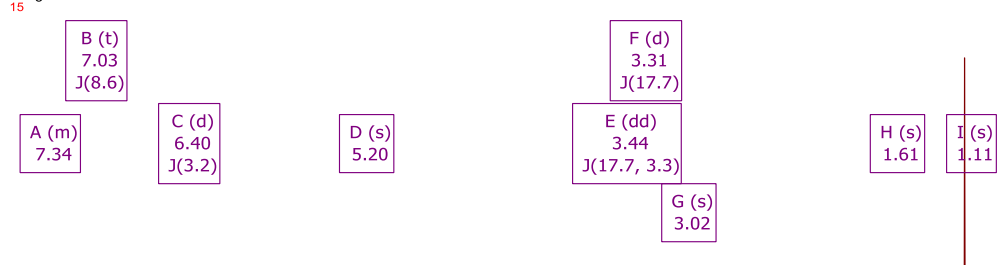

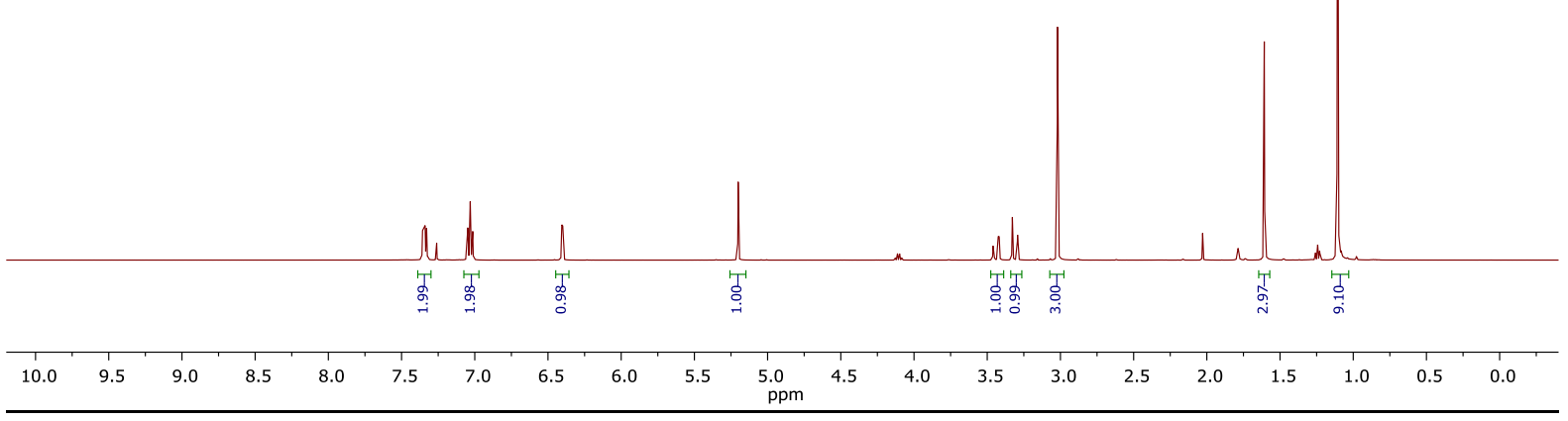

4c

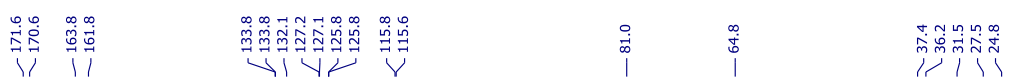

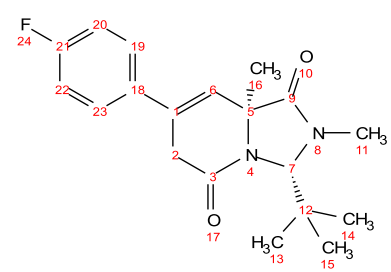

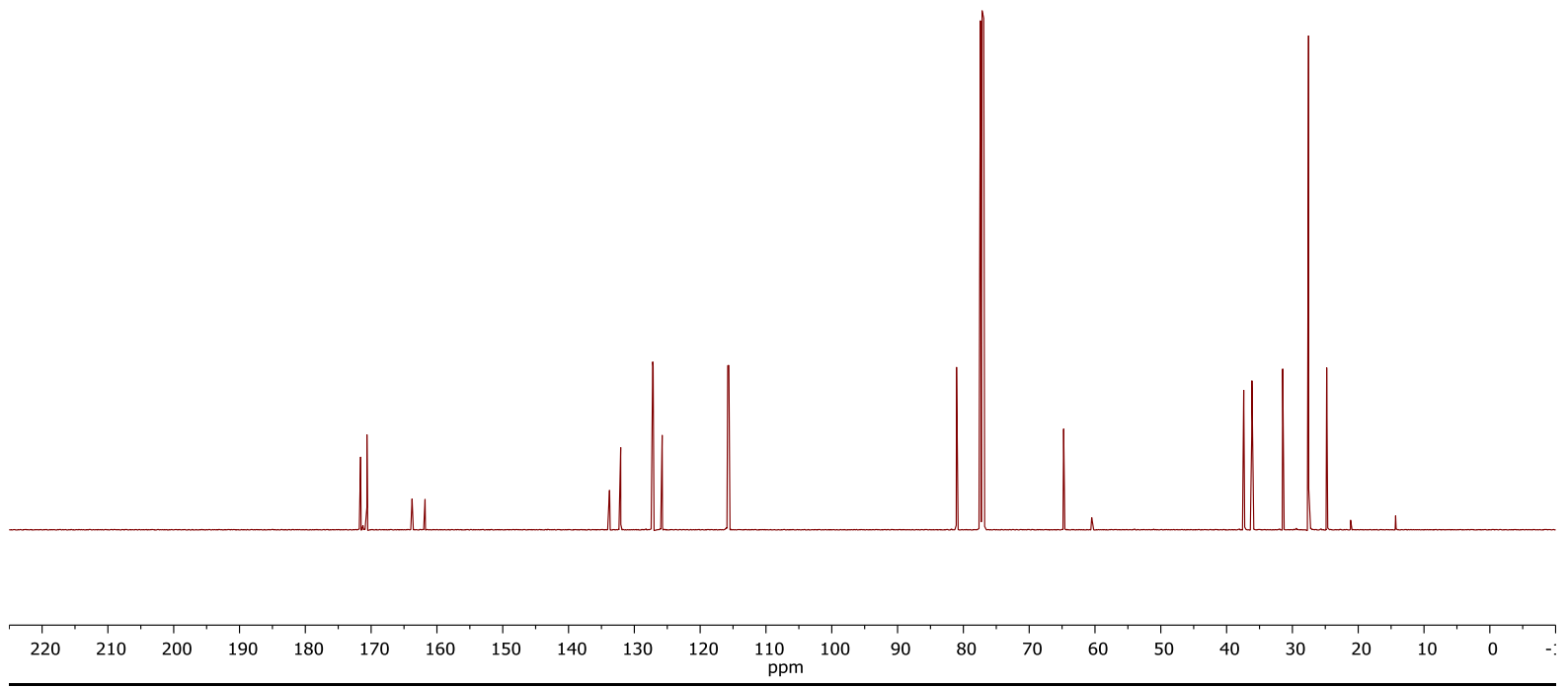


3d
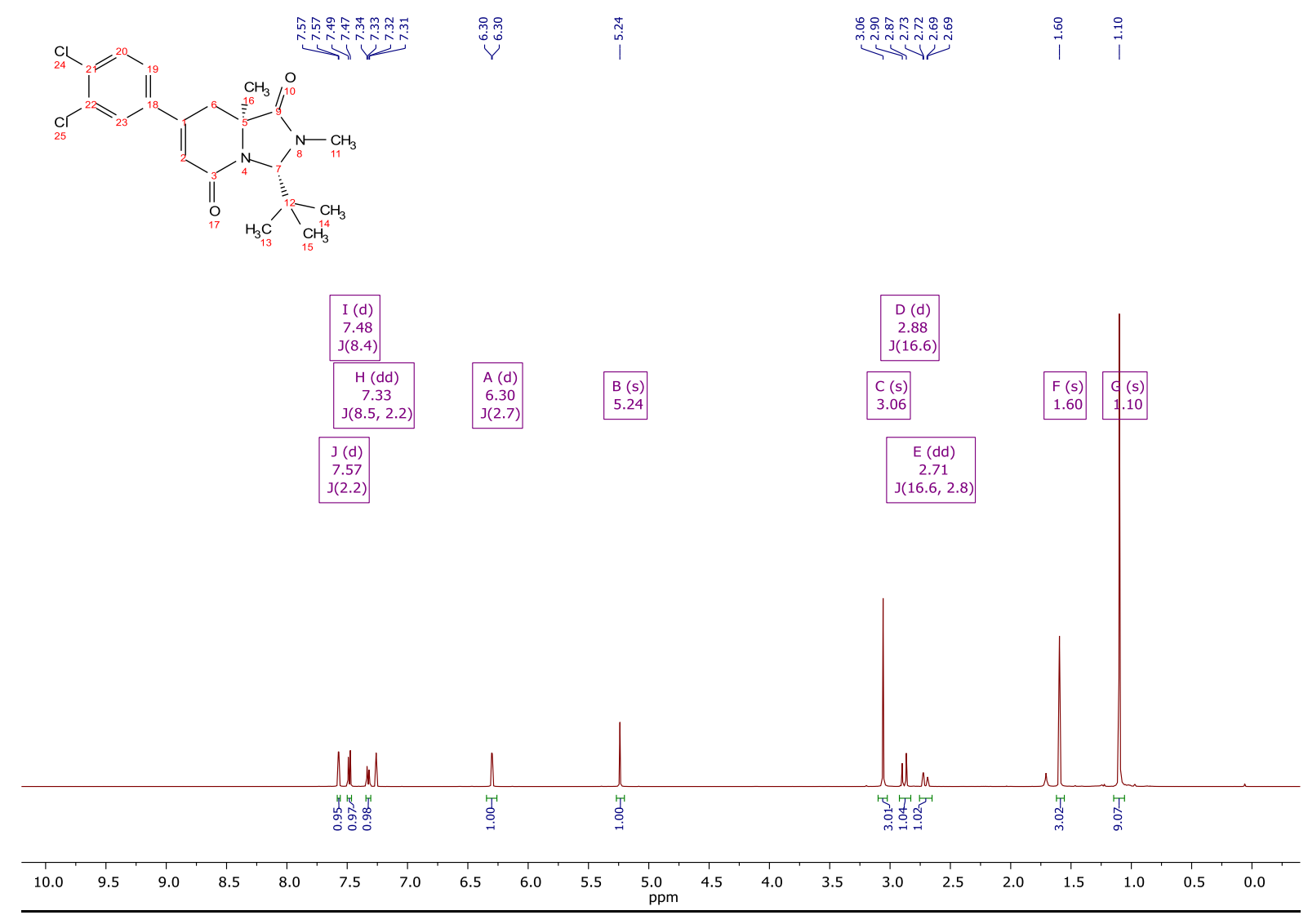

3d

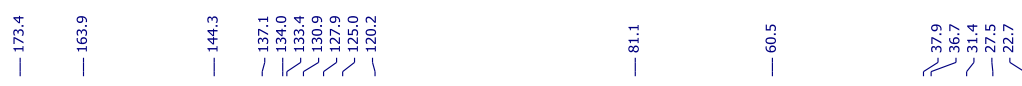

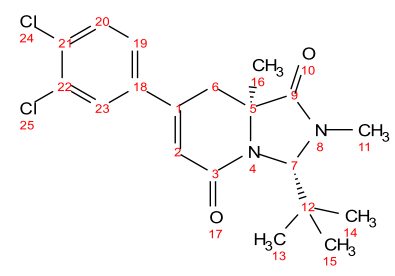

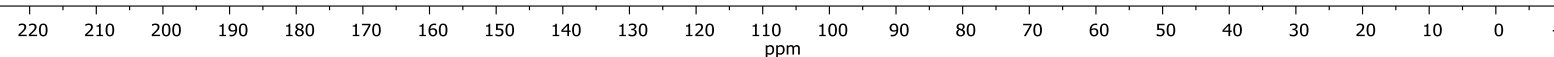


4d

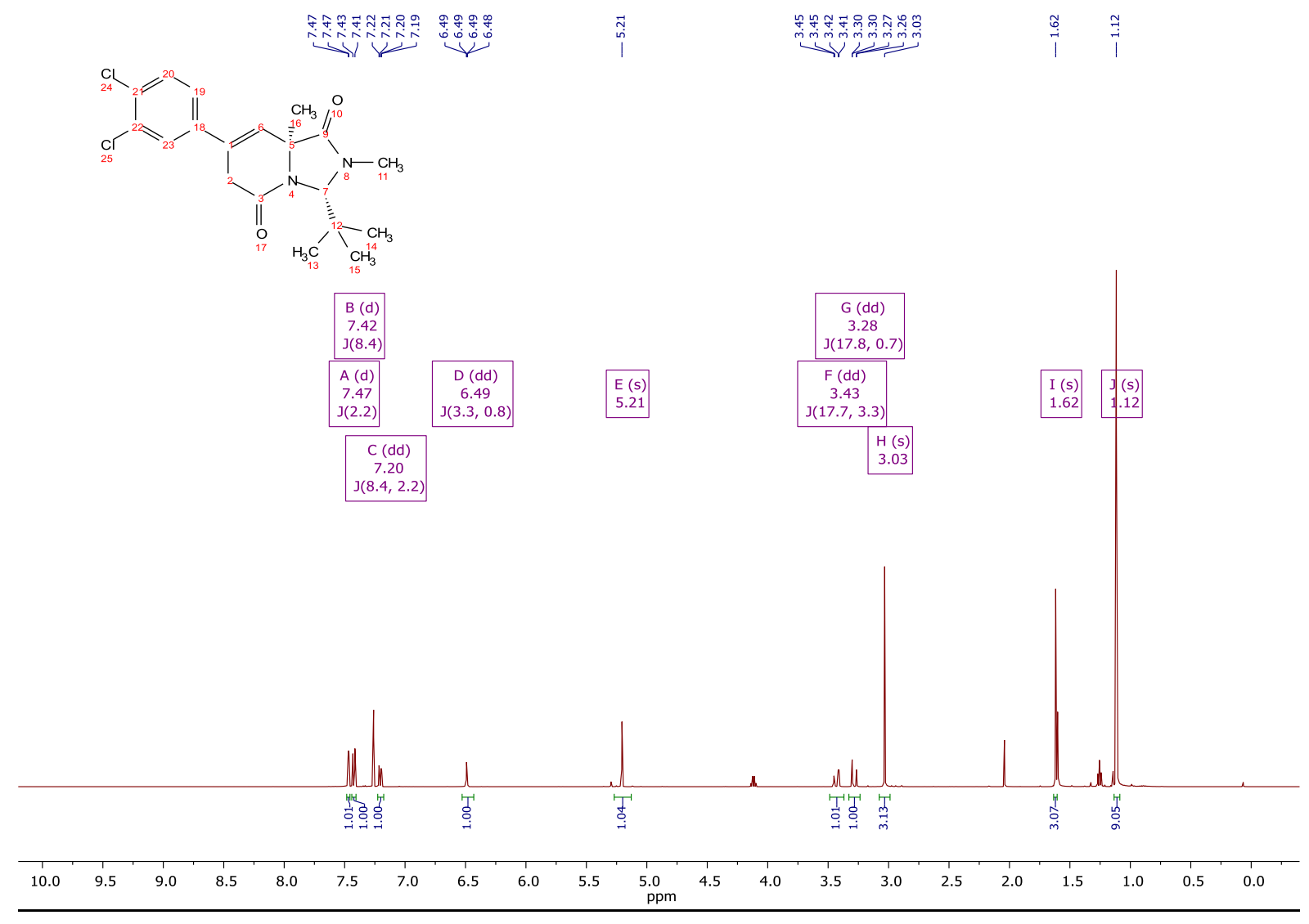

4d

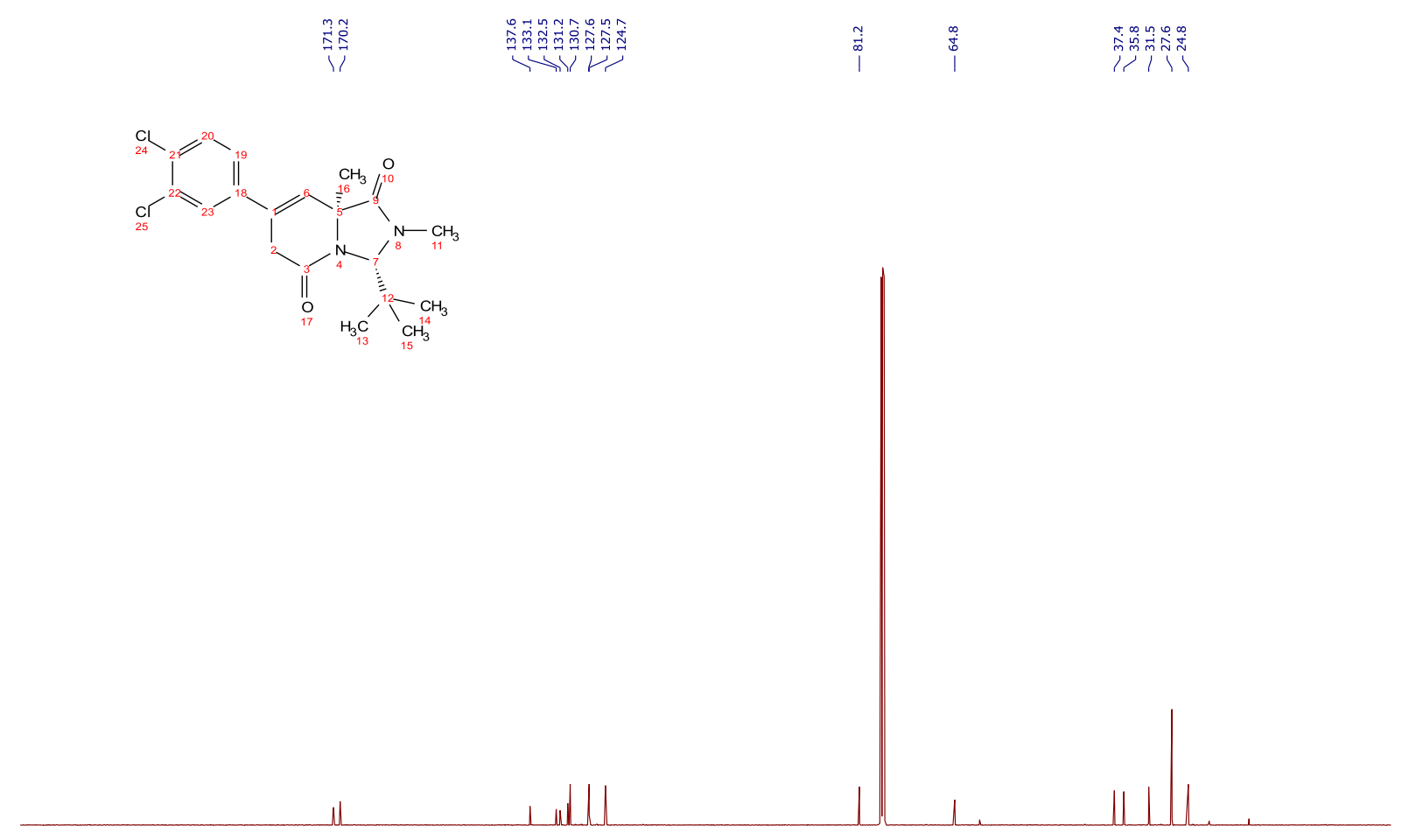

\begin{tabular}{lllllllllllllllllllllllll}
\hline 220 & 210 & 200 & 190 & 180 & 170 & 160 & 150 & 140 & 130 & 120 & 110 & 100 & 90 & 80 & 70 & 60 & 50 & 40 & 30 & 20 & 10 & 0 & -
\end{tabular} 
3e

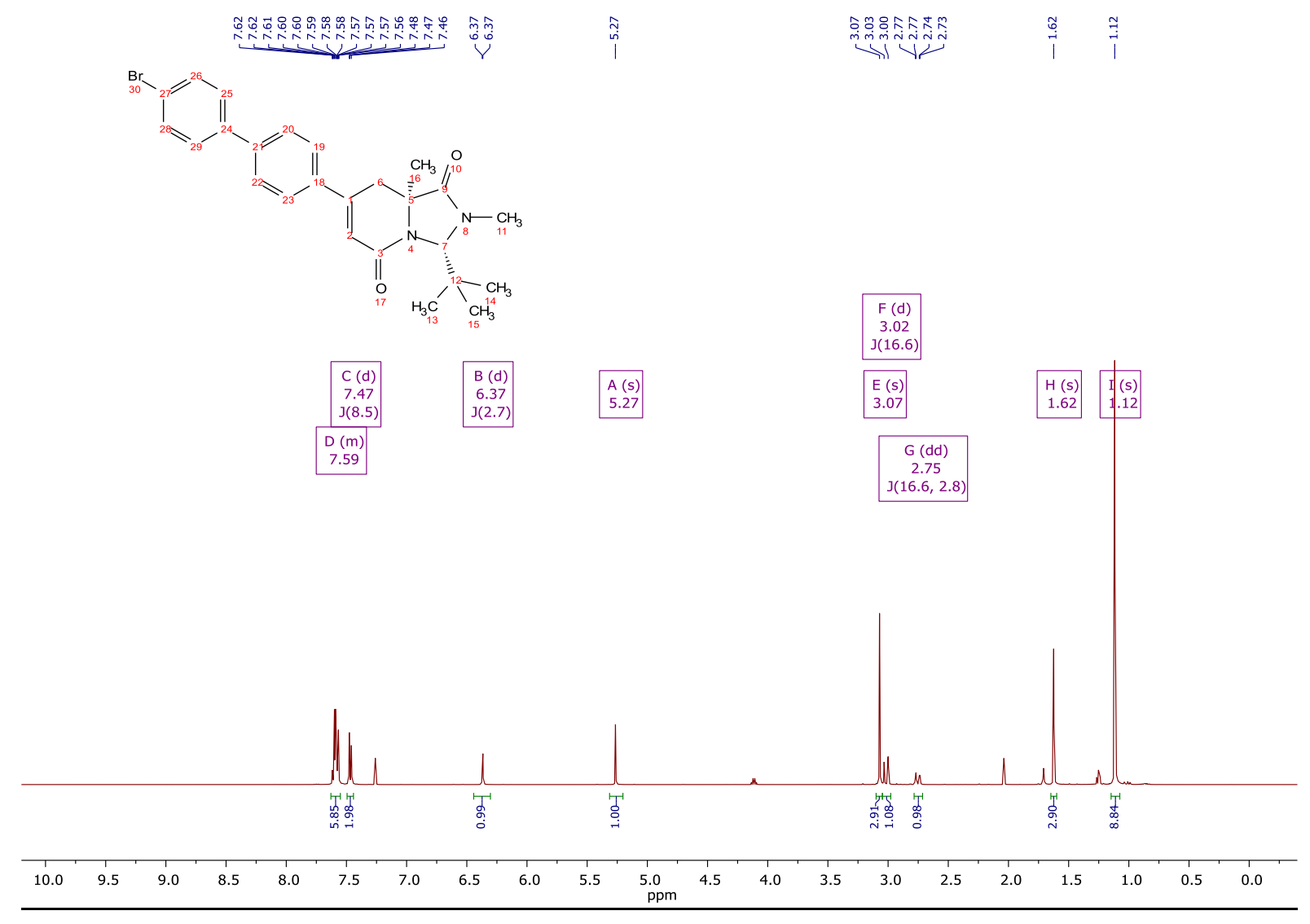

$3 e$

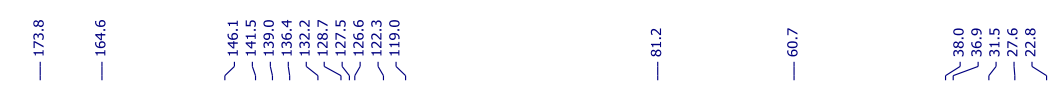
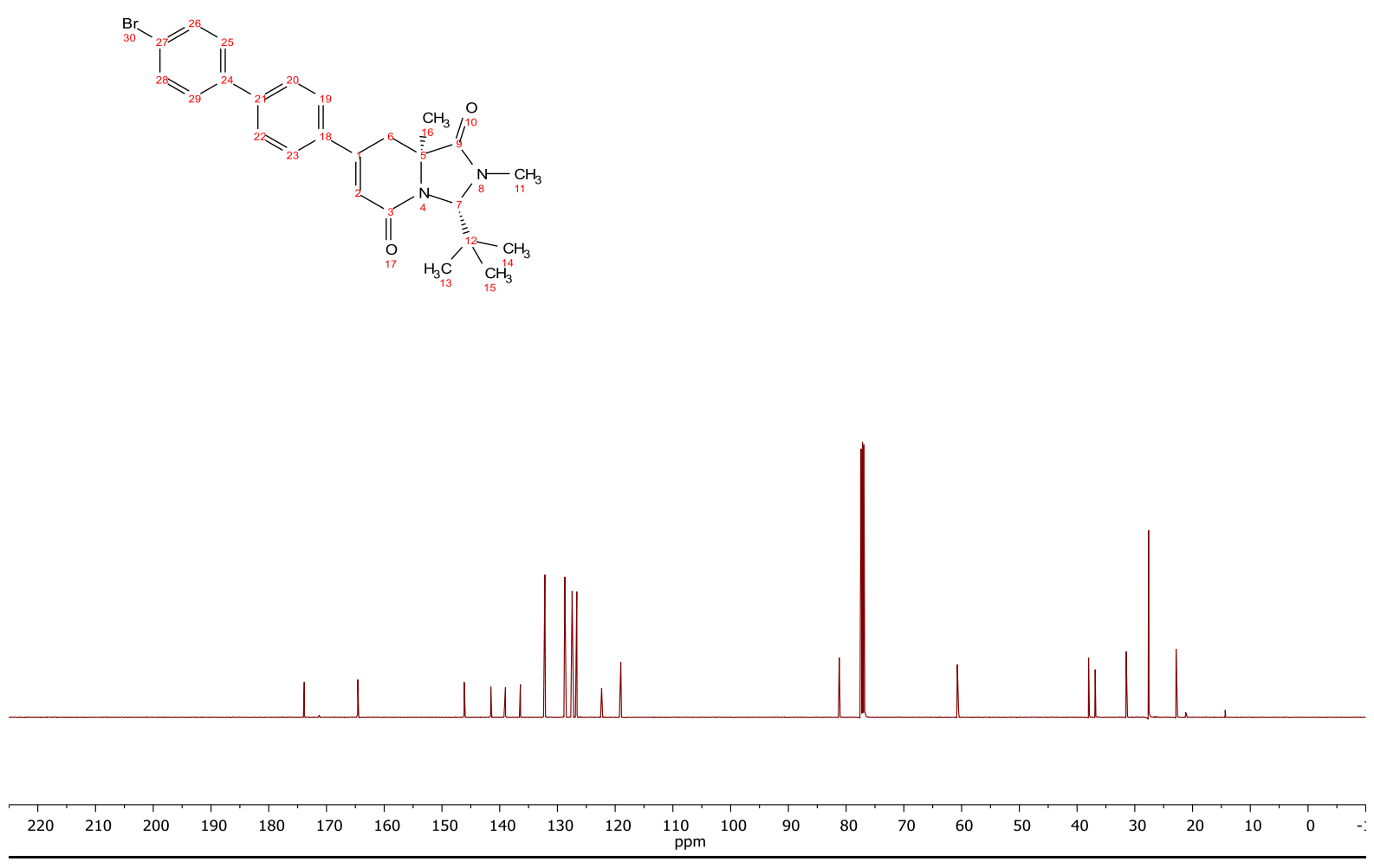
$4 e$
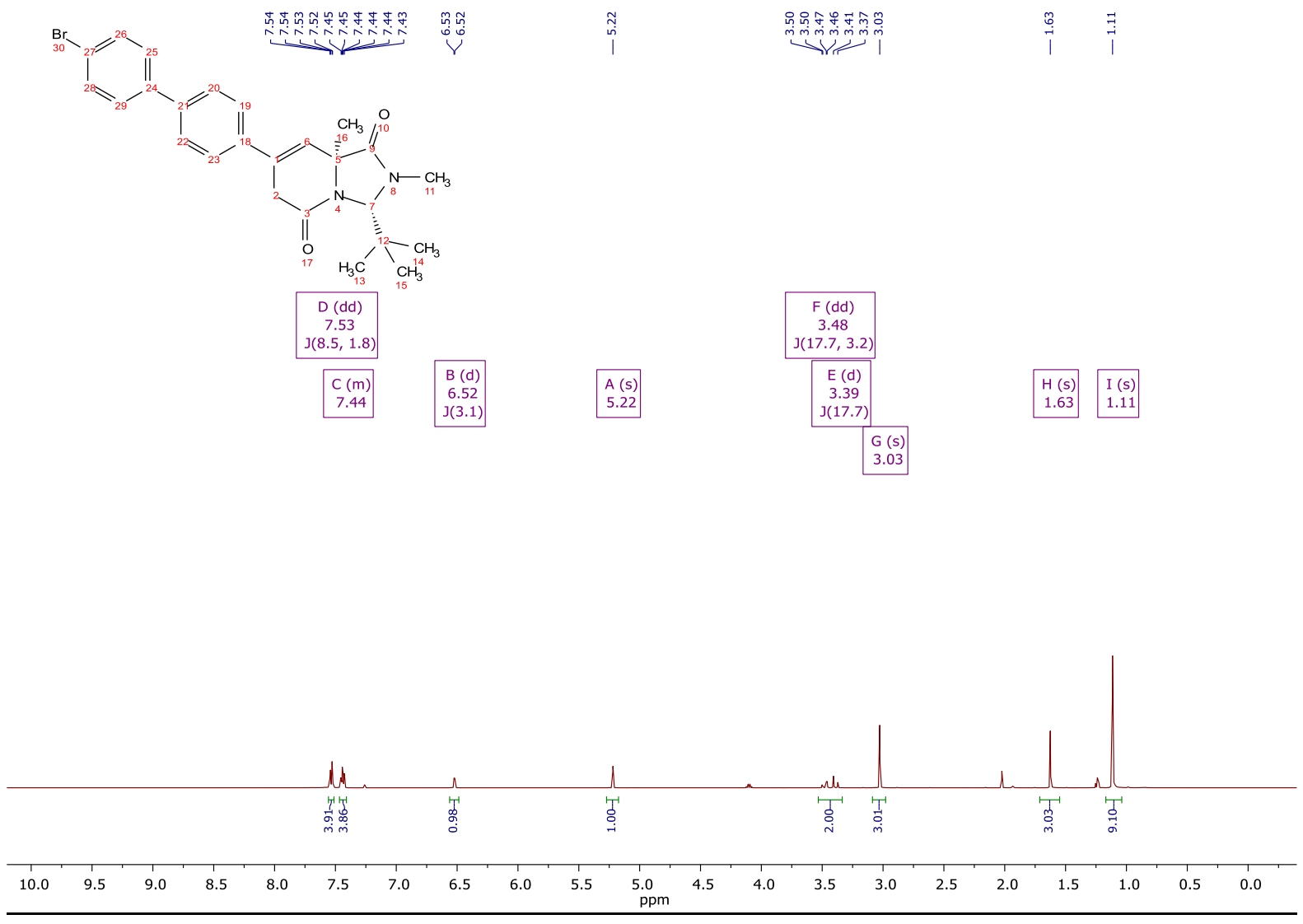

$4 e$

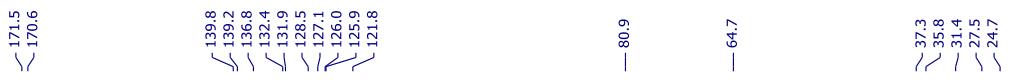
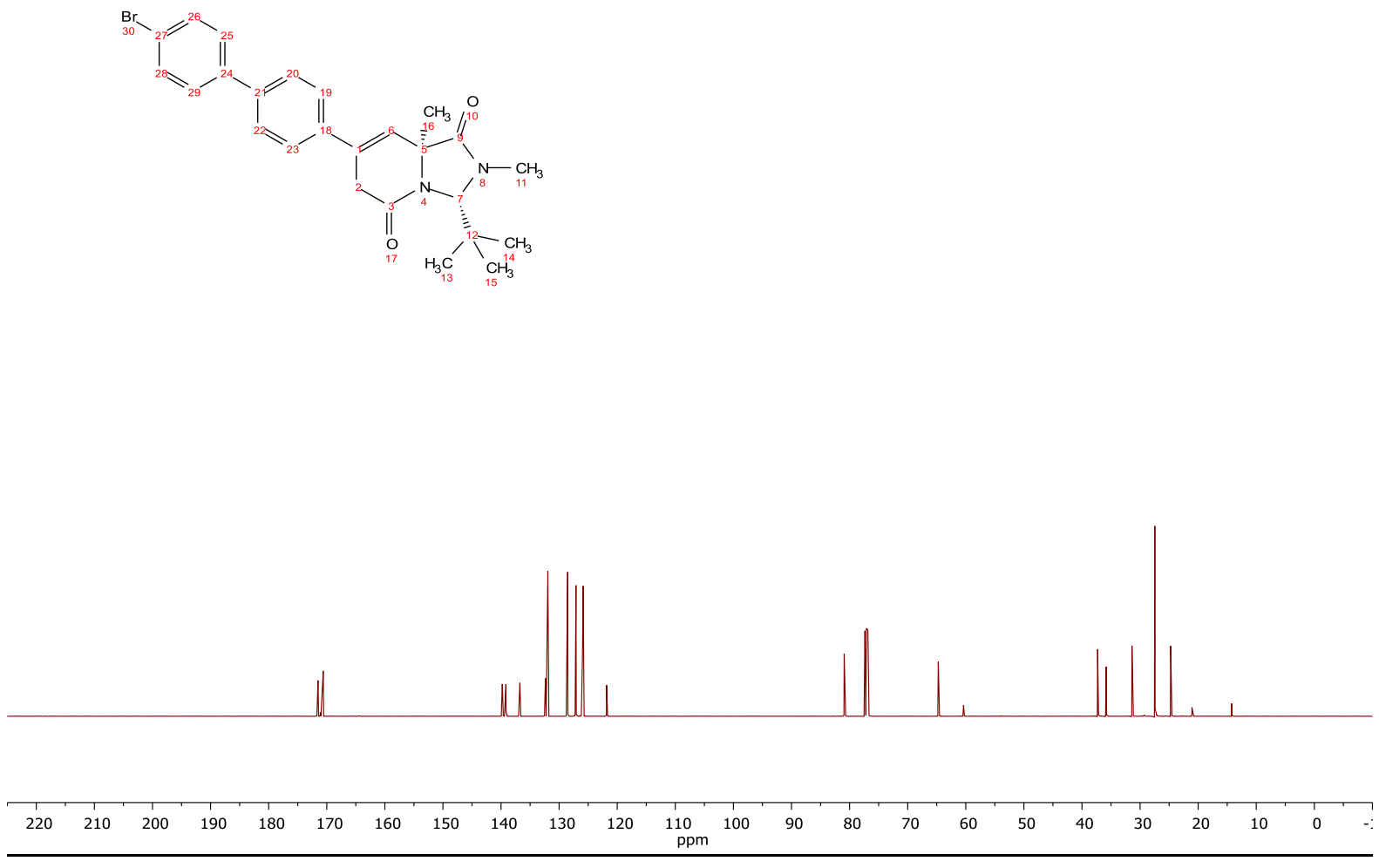
3k

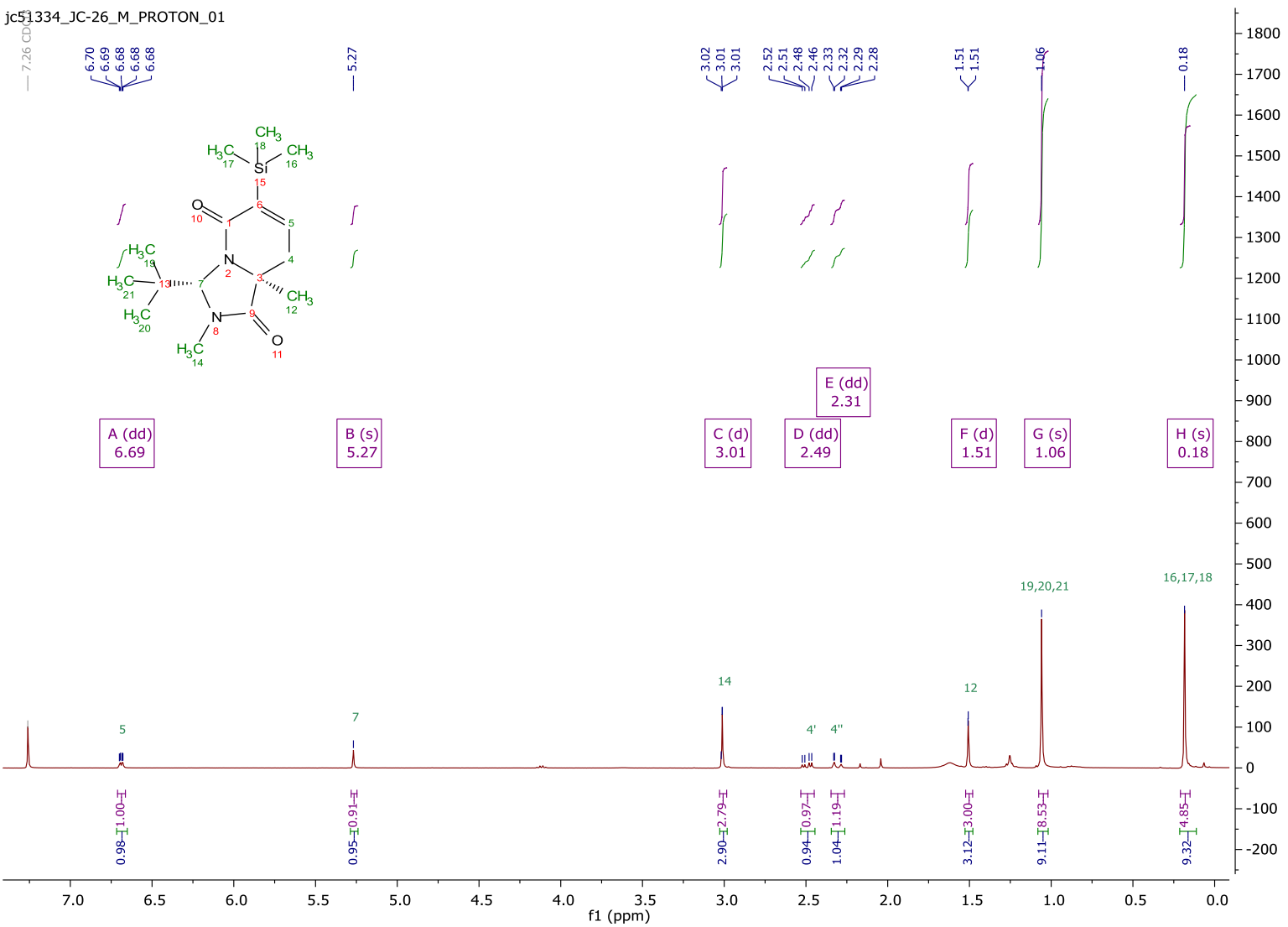

3k

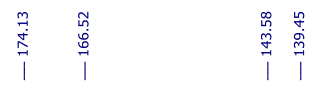
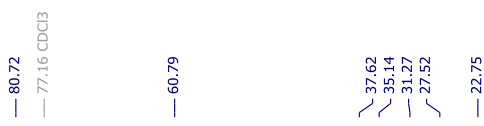

$\mid-11000$

10000

9000

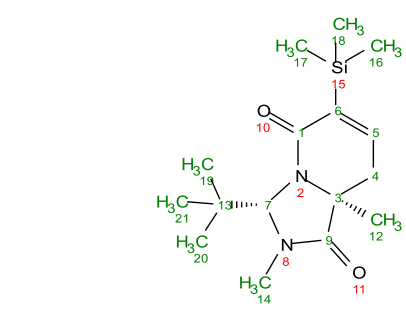

$\mathrm{CH}_{3} \mathrm{CH}_{3}$

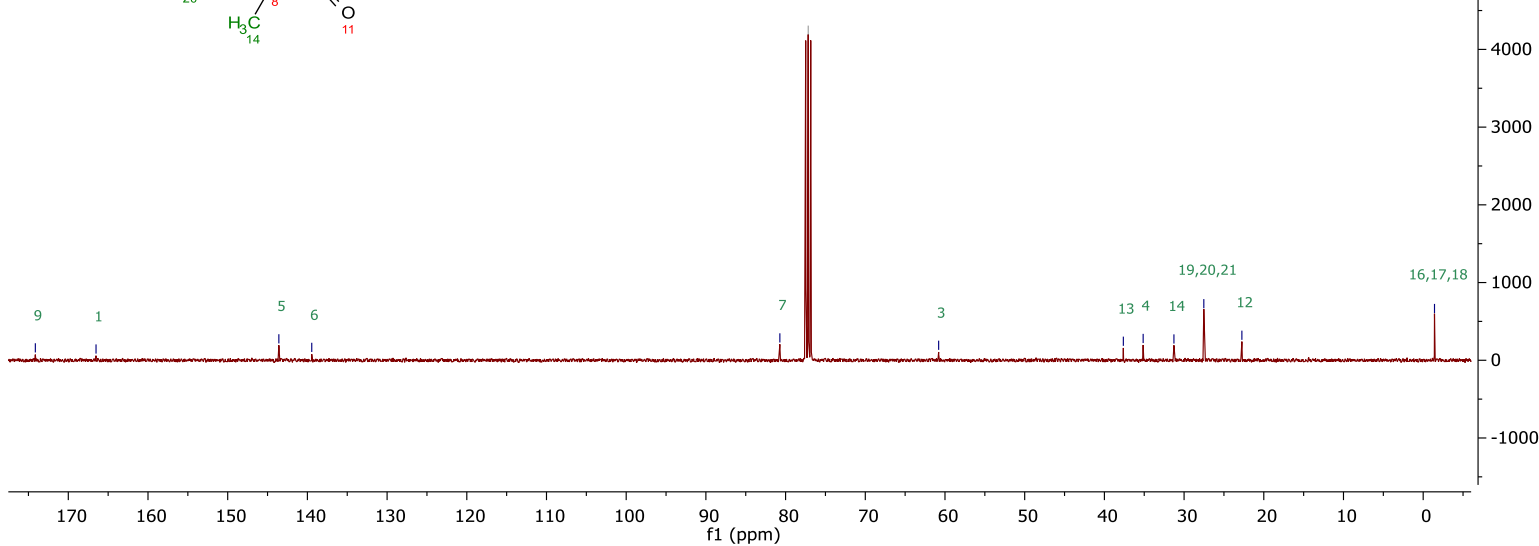


$7 k$

39496 JC-26 top full charac.10.fid ن

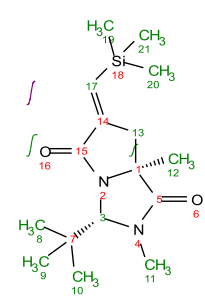

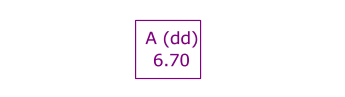

\begin{tabular}{|l|}
\hline$B(s)$ \\
4.79 \\
\hline
\end{tabular}

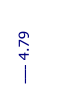

1

1)
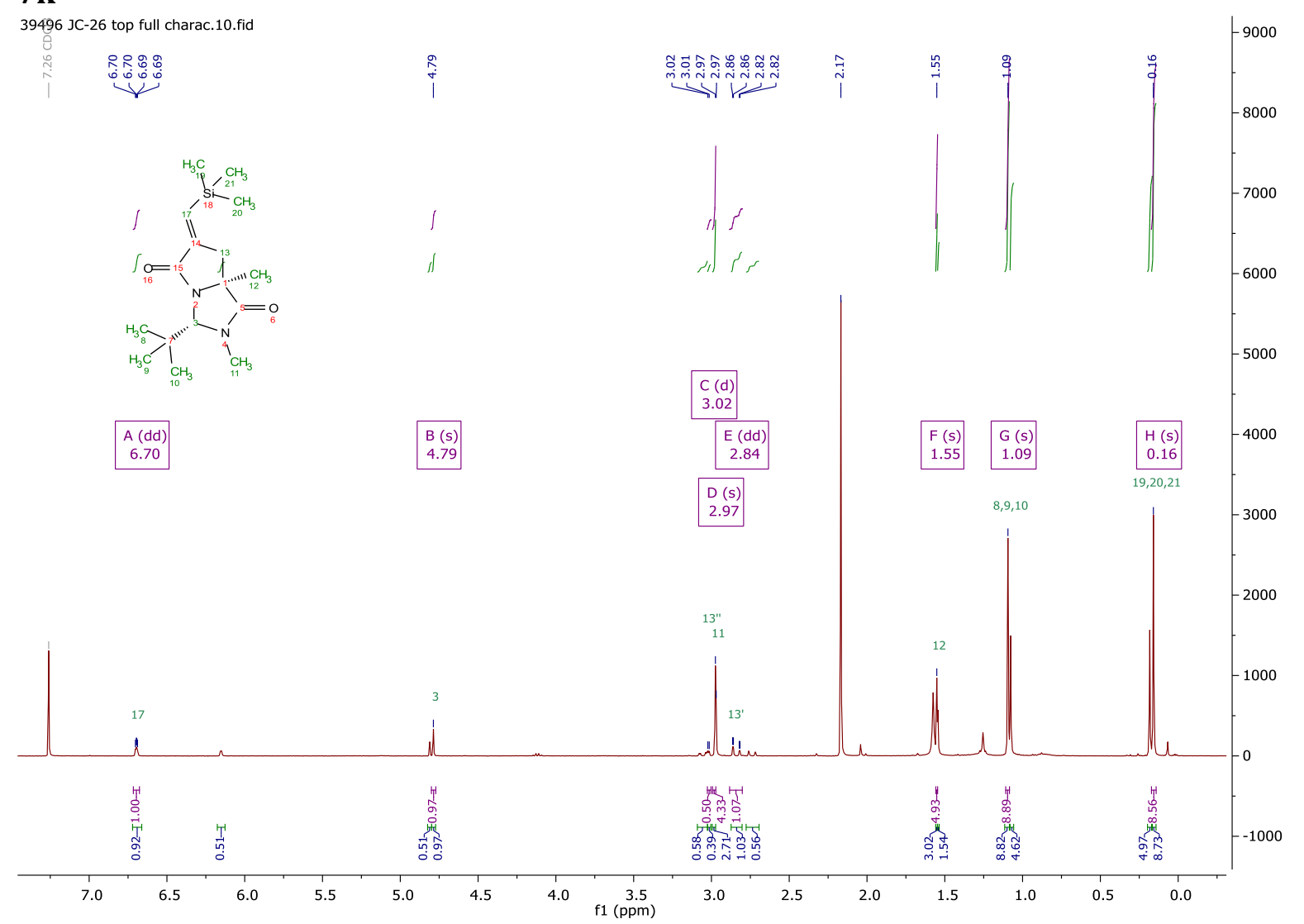

7k

39496 JC-26 top full charac.14.fid 莒

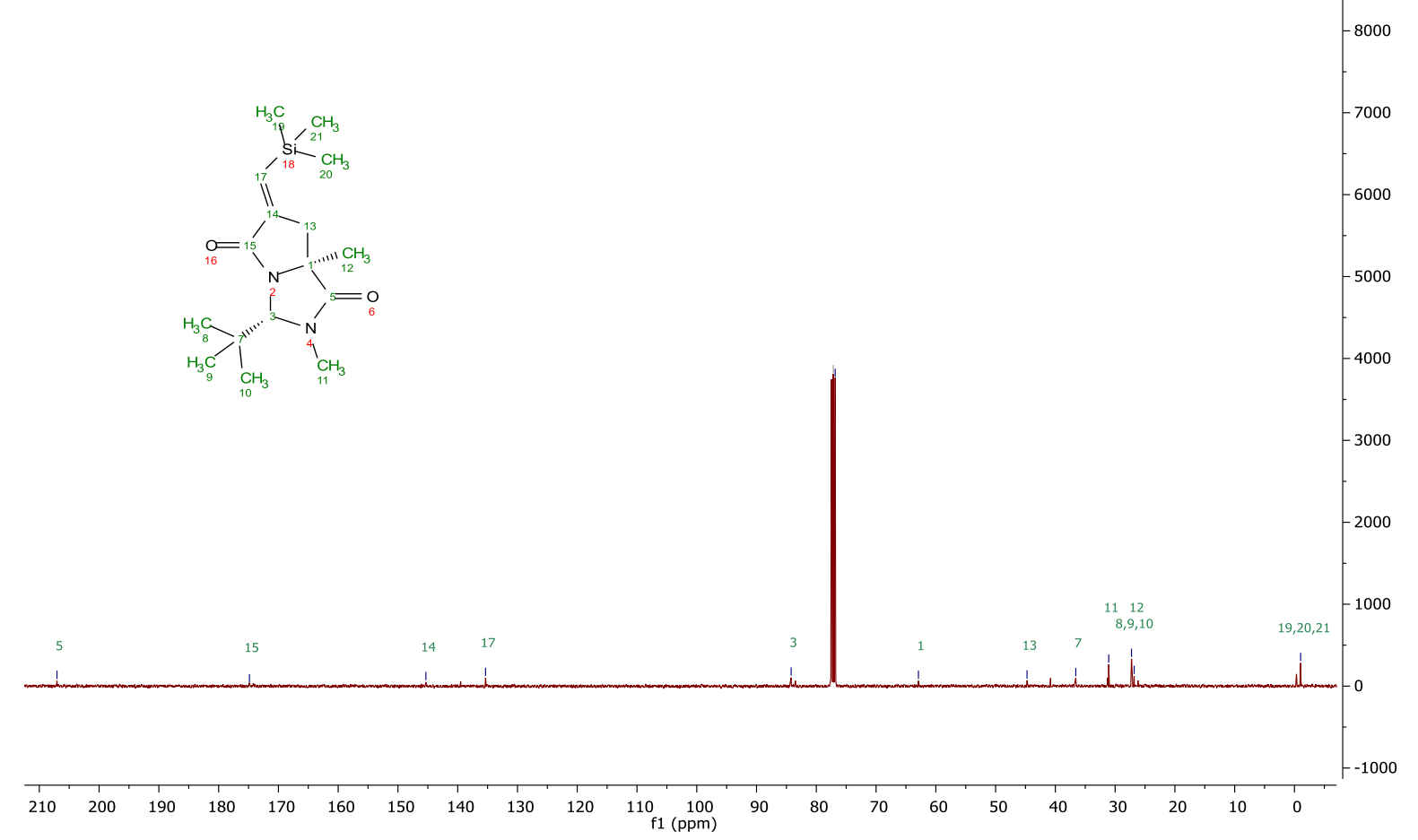




\section{3f}

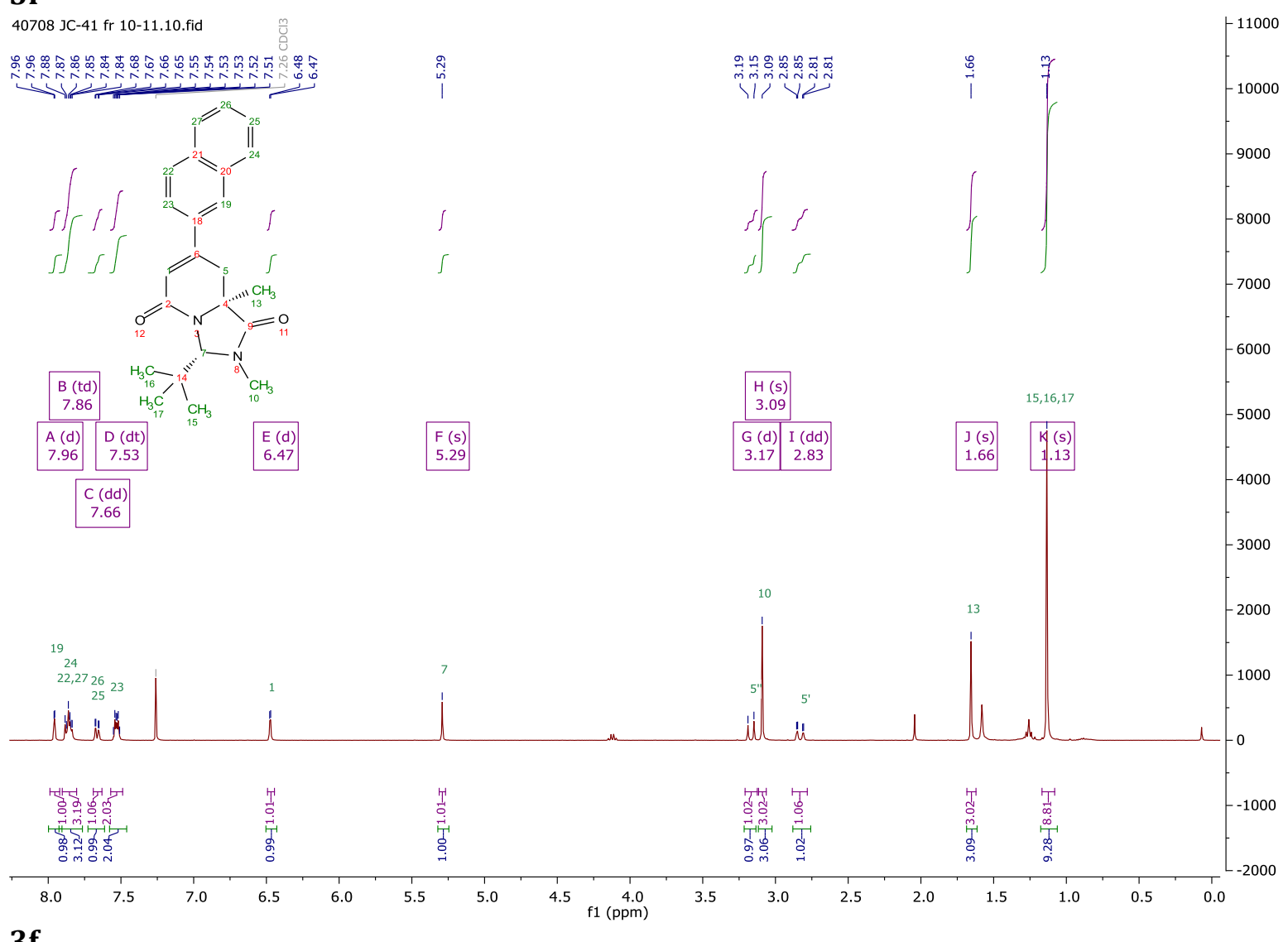

3f

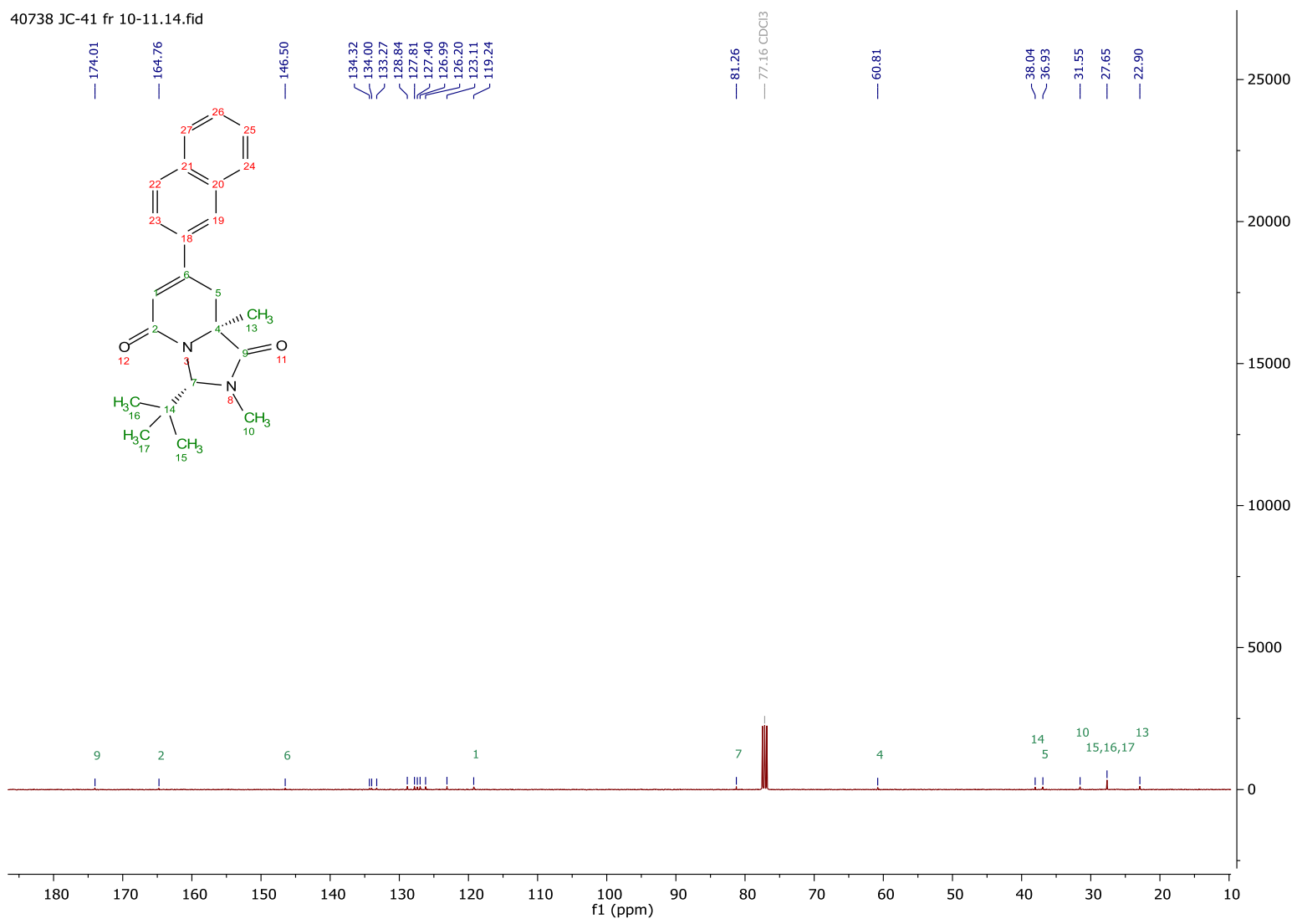


4f

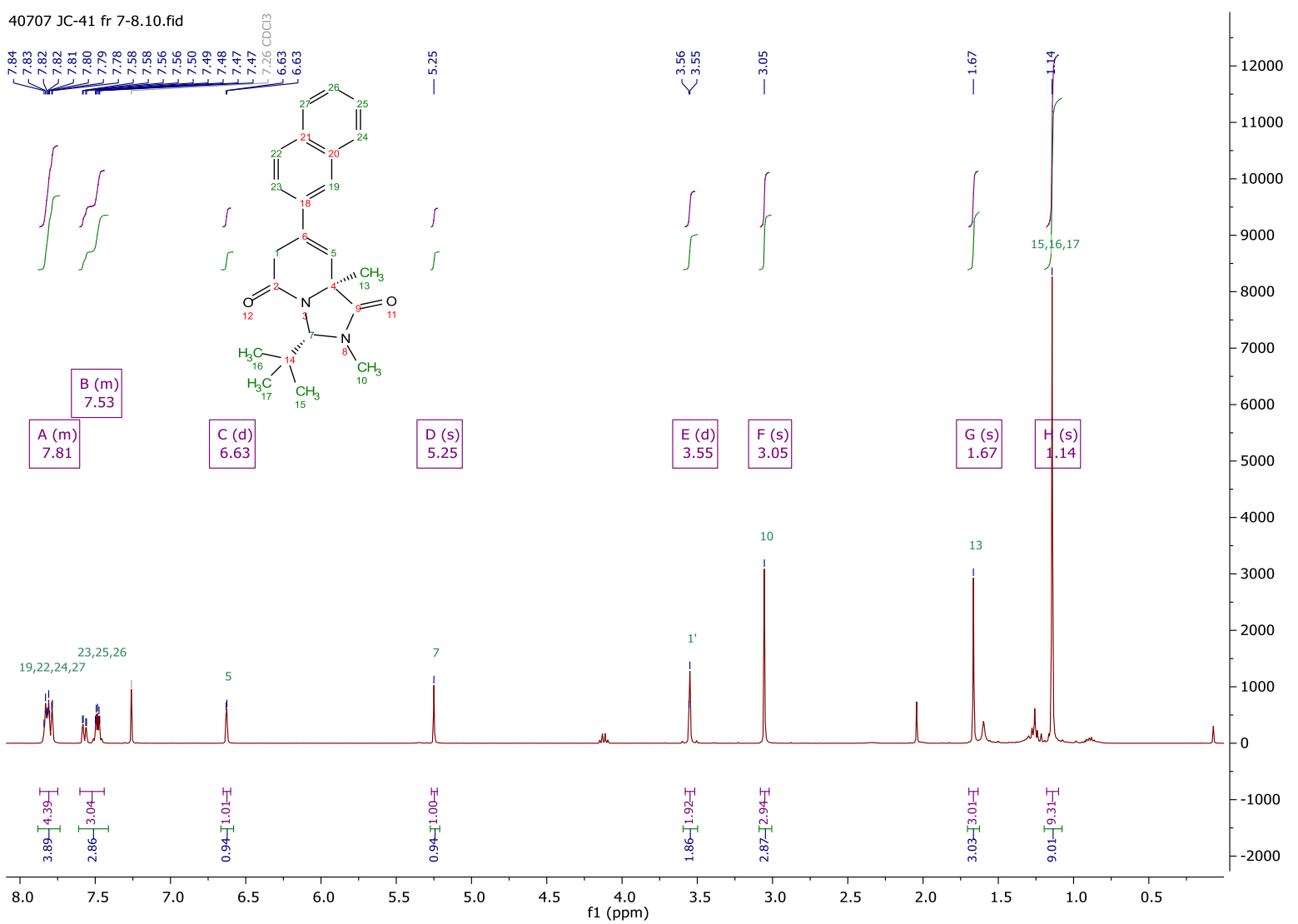

4f

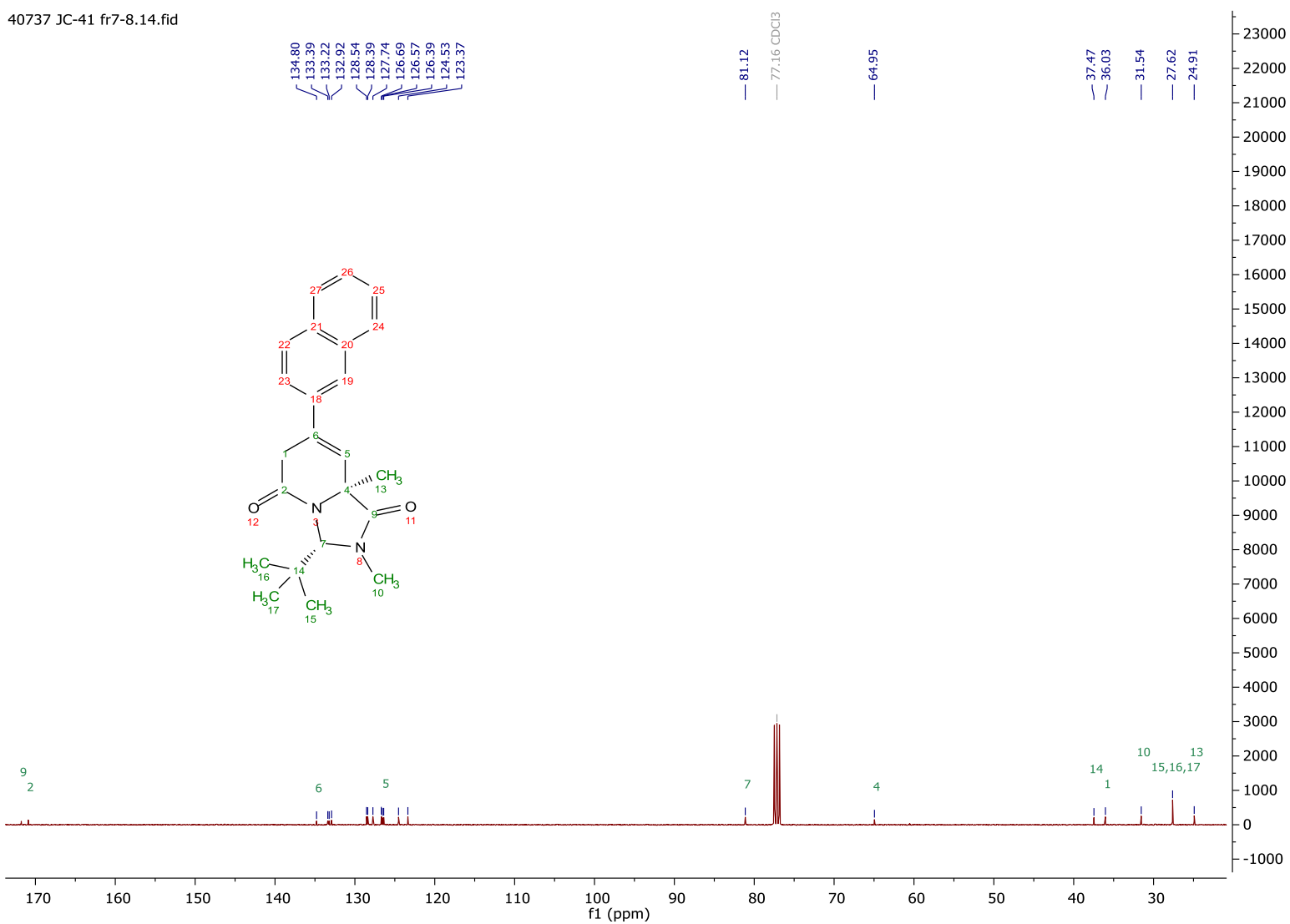




\section{$3 g$}

$\underbrace{\underbrace{20}_{10}}_{2=10}$<smiles></smiles>

$3($ ddd)
6.96

\begin{tabular}{|c|c|c|c|c|c|c|}
\hline$A(t)$ & $E(d)$ & $F(s)$ & $G(s)$ & $J(s)$ & $K(s)$ & $L(s)$ \\
7.26 & 6.46 & 3.80 & 3.02 & \\
$J(8.0)$ & 5.21 & 1.61 & 1.11 \\
\hline$J(3.7)$ & & & \\
\hline
\end{tabular}

\begin{tabular}{|r|c|}
\hline $\mathrm{C}(\mathrm{t})$ & $\mathrm{I}(\mathrm{dd})$ \\
6.89 & 3.35 \\
$\mathrm{~J}(2.1)$ & $\mathrm{J}(17.8,0.6)$ \\
\hline
\end{tabular}

d.

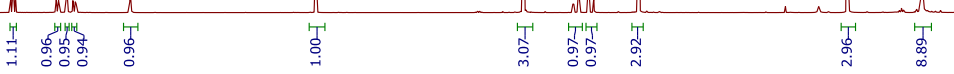

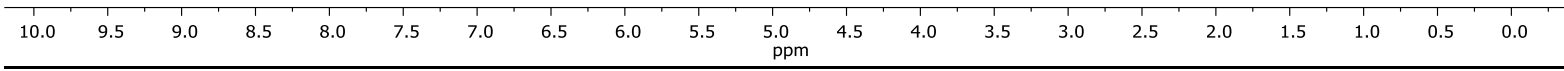

$3 g$

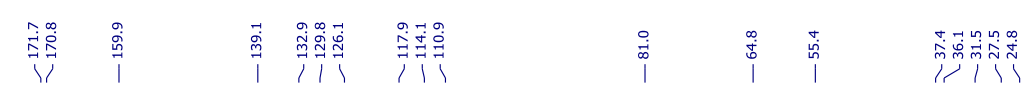

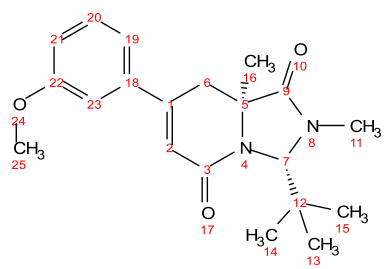

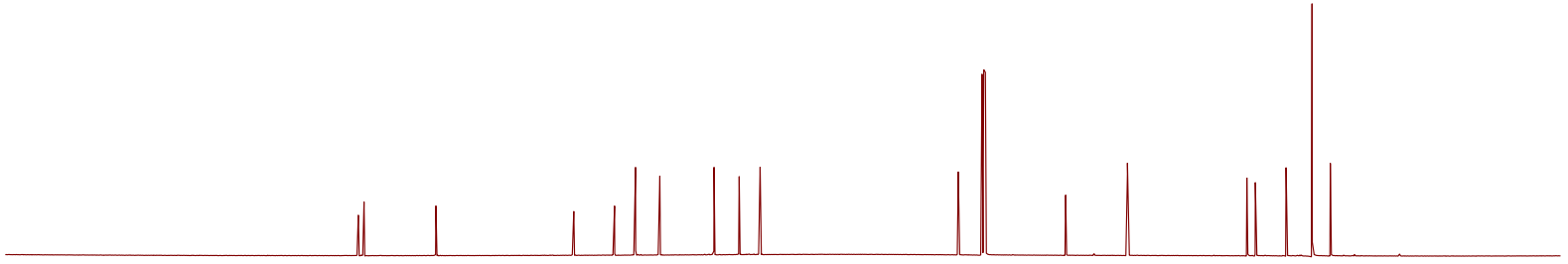

$\begin{array}{llllllllllllllllllllllllllllll}220 & 210 & 200 & 190 & 180 & 170 & 160 & 150 & 140 & 130 & 120 & 110 & 100 & 90 & 80 & 70 & 60 & 50 & 40 & 30 & 20 & 10 & 0 & -\end{array}$ 
$4 \mathrm{~g}$

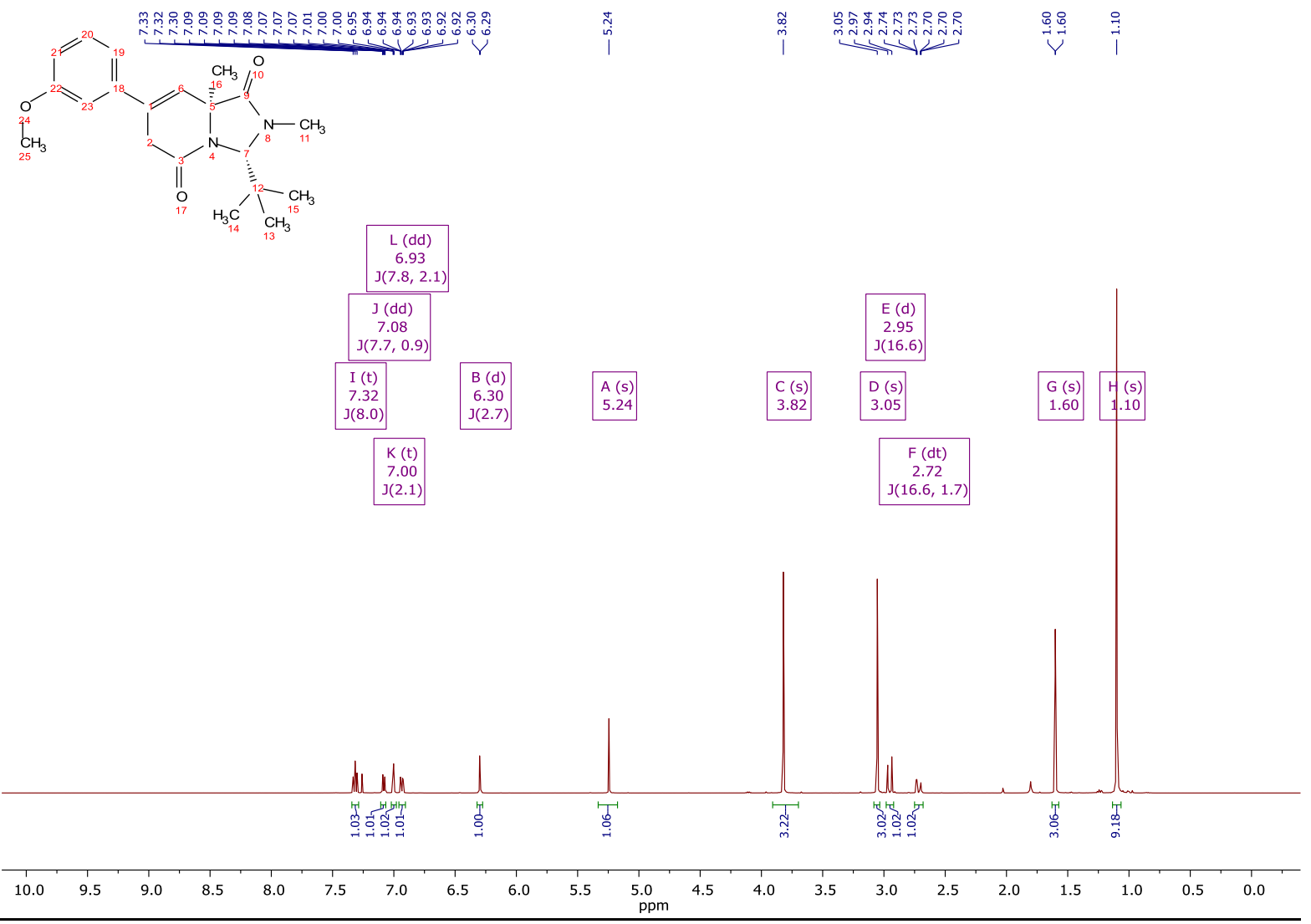

$4 \mathrm{~g}$

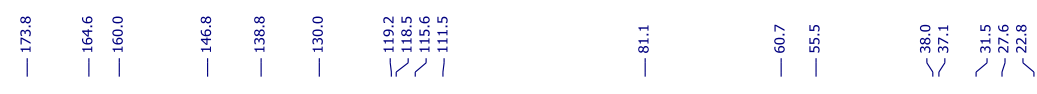

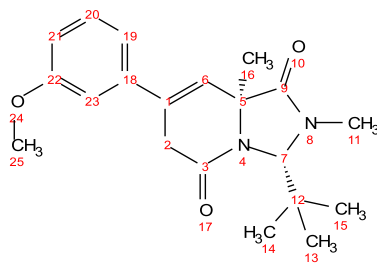

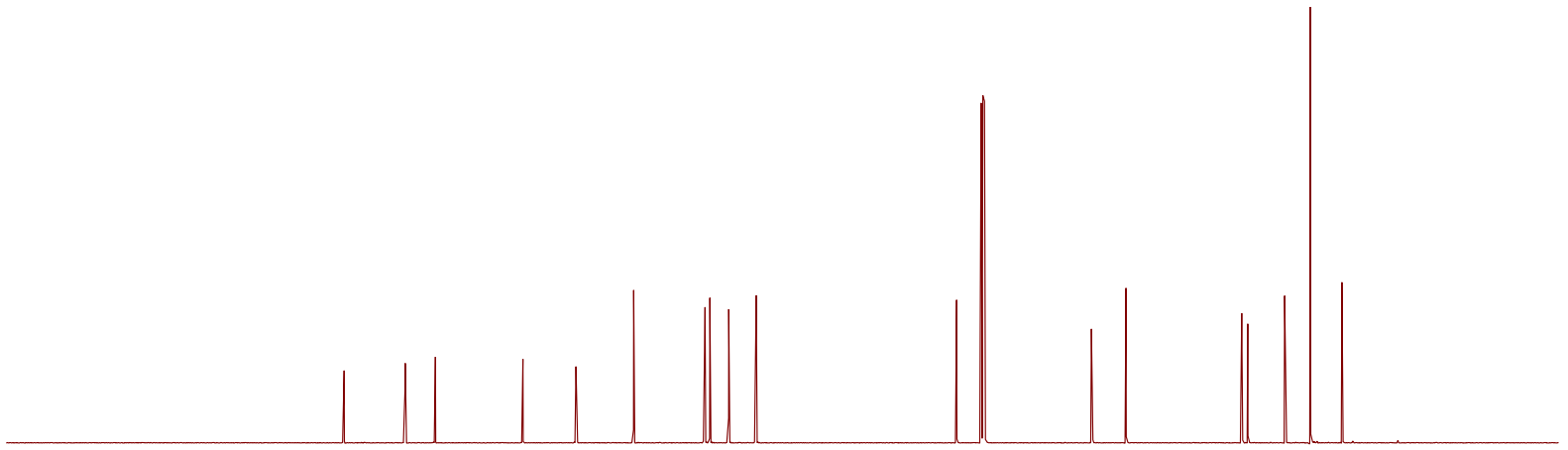

$\begin{array}{lllllllllllllllllllllllllllllllllll}220 & 210 & 200 & 190 & 180 & 170 & 160 & 150 & 140 & 130 & 120 & 110 & 100 & 90 & 80 & 70 & 60 & 50 & 40 & 30 & 20 & 10 & 0 & \end{array}$ 
3j

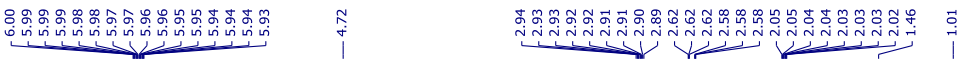
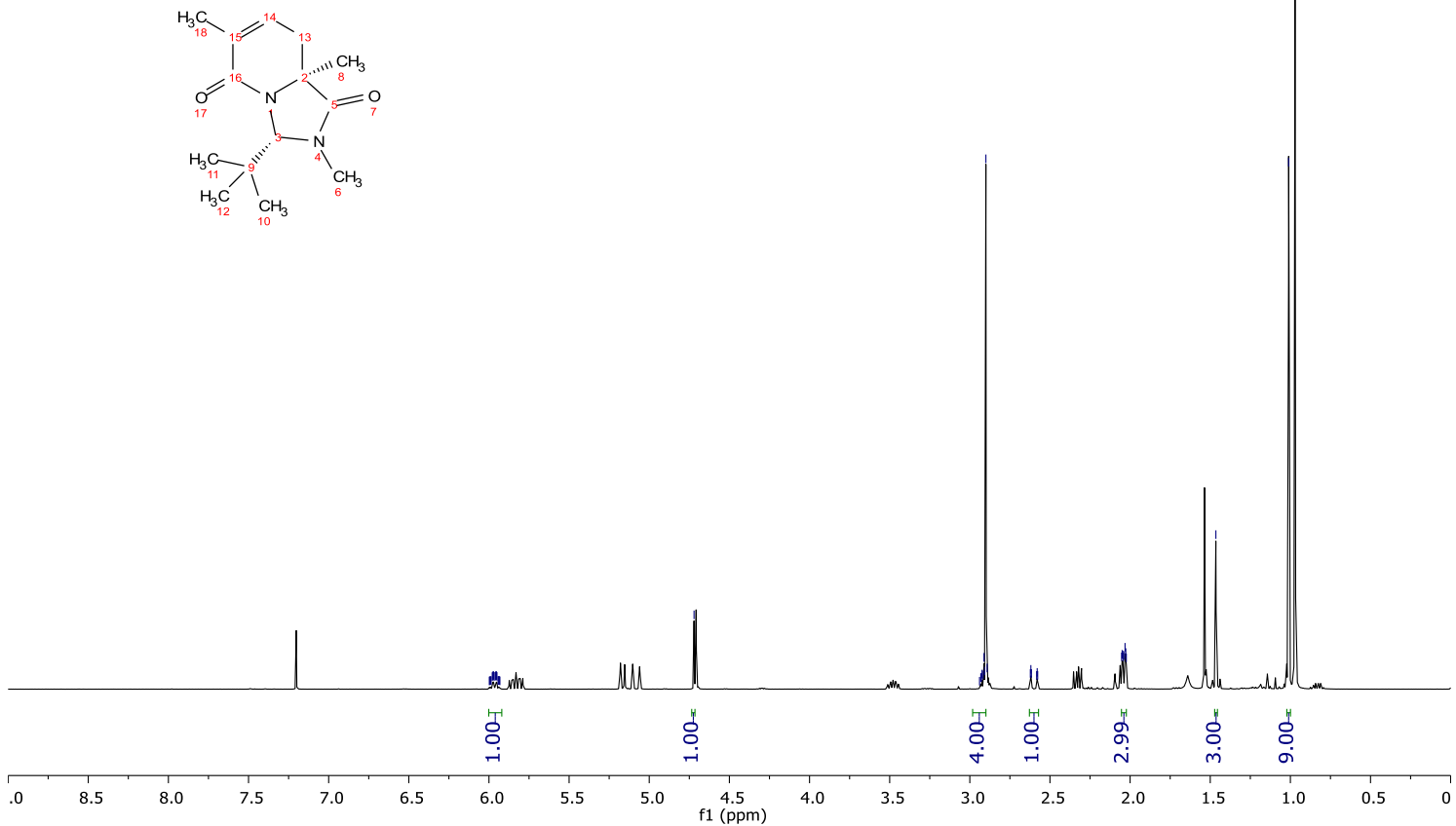

3j

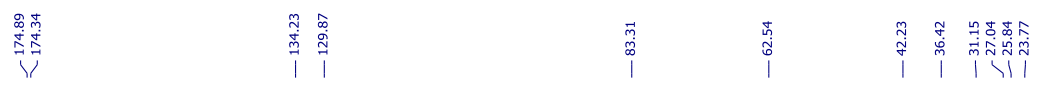

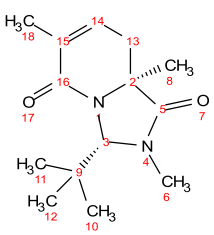

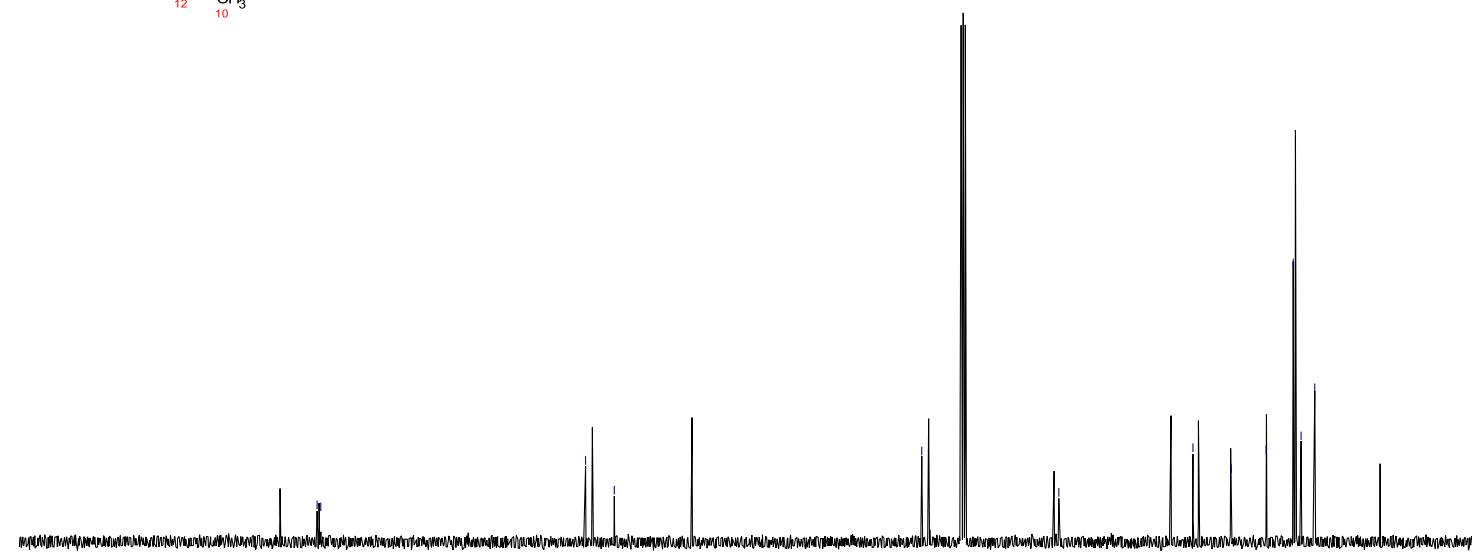

$\begin{array}{llllllllllllllllllllllllllll}210 & 200 & 190 & 180 & 170 & 160 & 150 & 140 & 130 & 120 & 110 & 100 & 90 & 80 & 70 & 60 & 50 & 40 & 30 & 20 & 10 & 1\end{array}$ 
3h

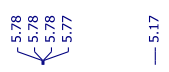

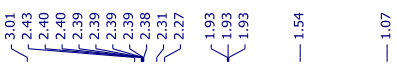

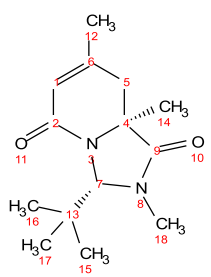

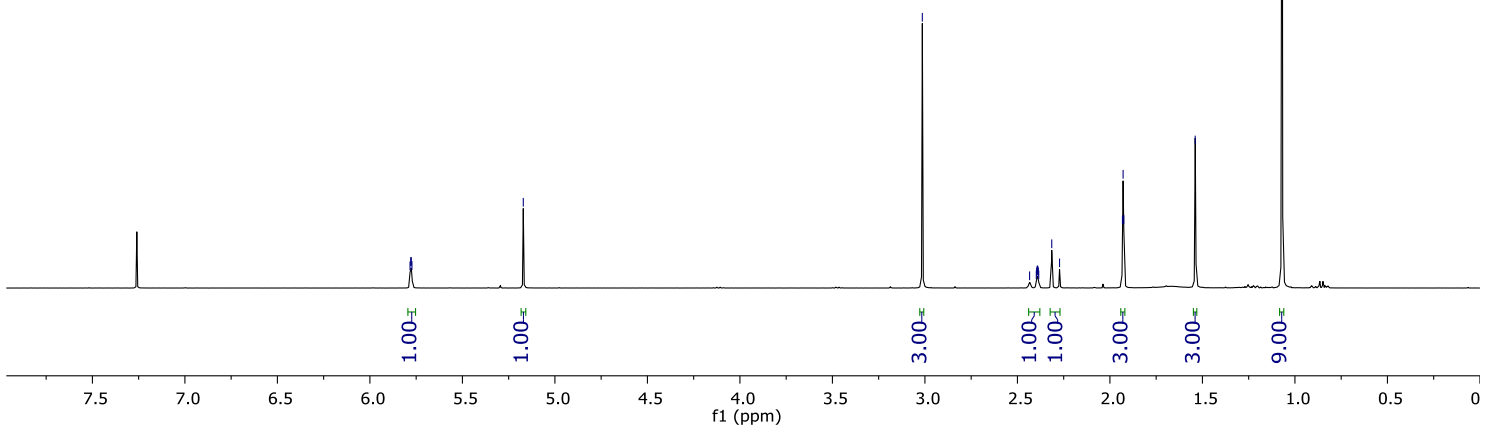

3h

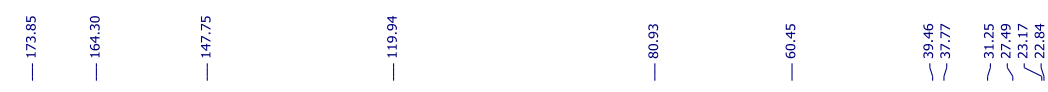

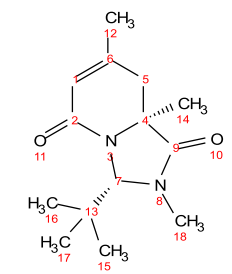

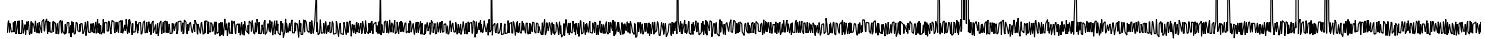

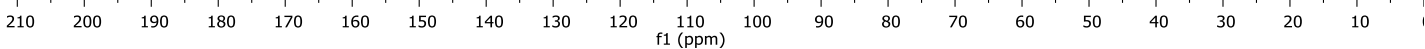


$4 h$

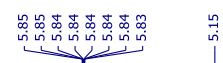

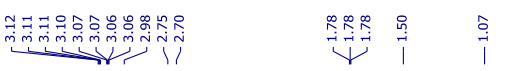<smiles>C=C1CC(O)CC2(C)N1C(=O)C(=O)N2C1CC1</smiles>

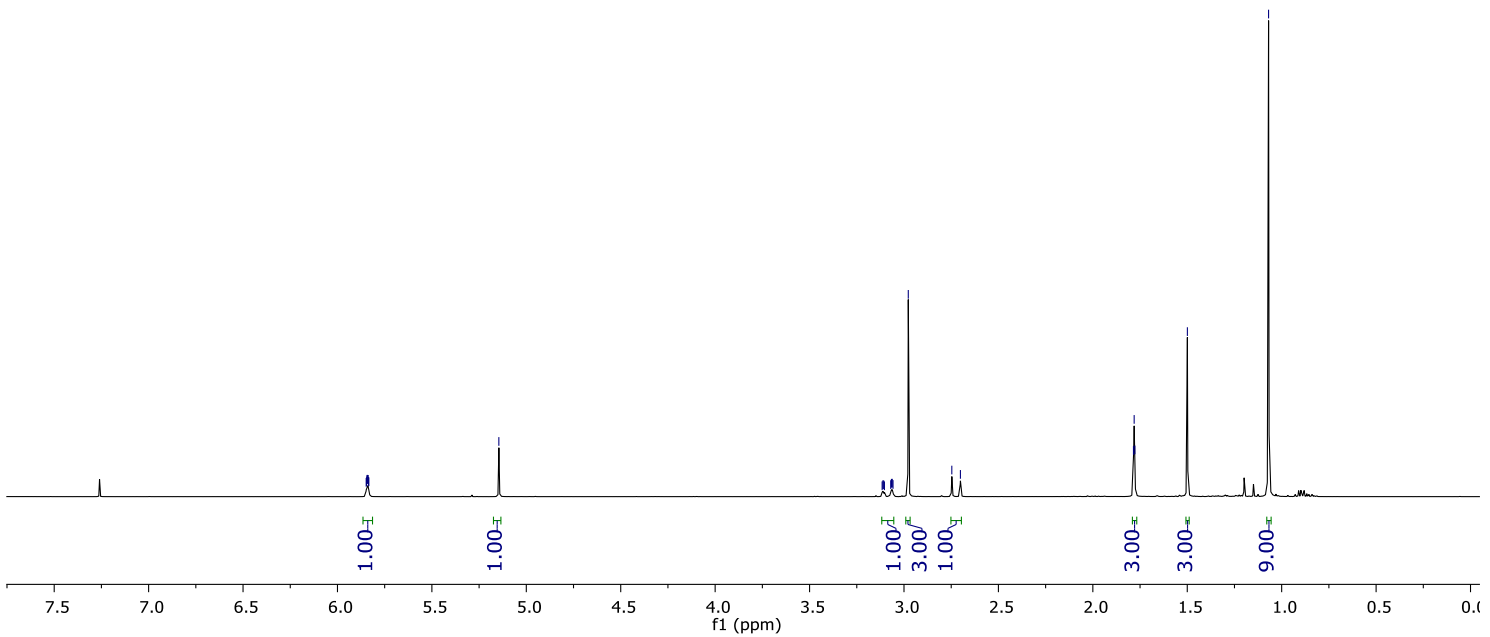

$4 h$

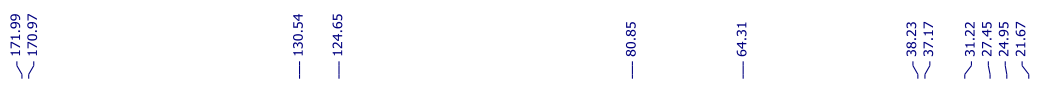<smiles>C=C1CC(O)CC2(C)C1N(C1CC1)C(=O)N2C</smiles>

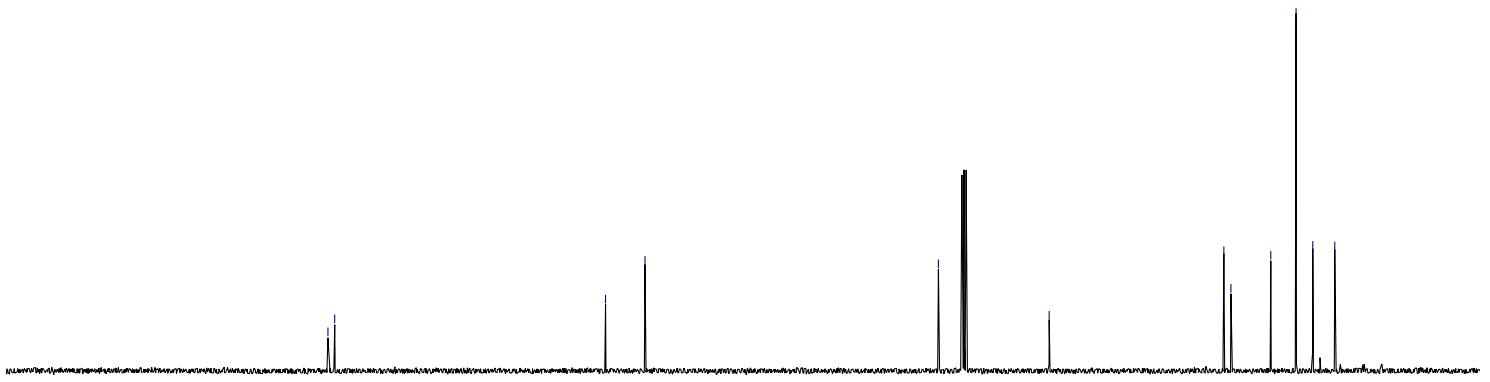

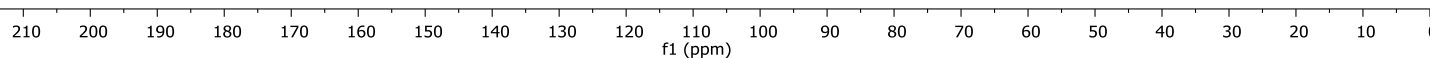


$6 h$
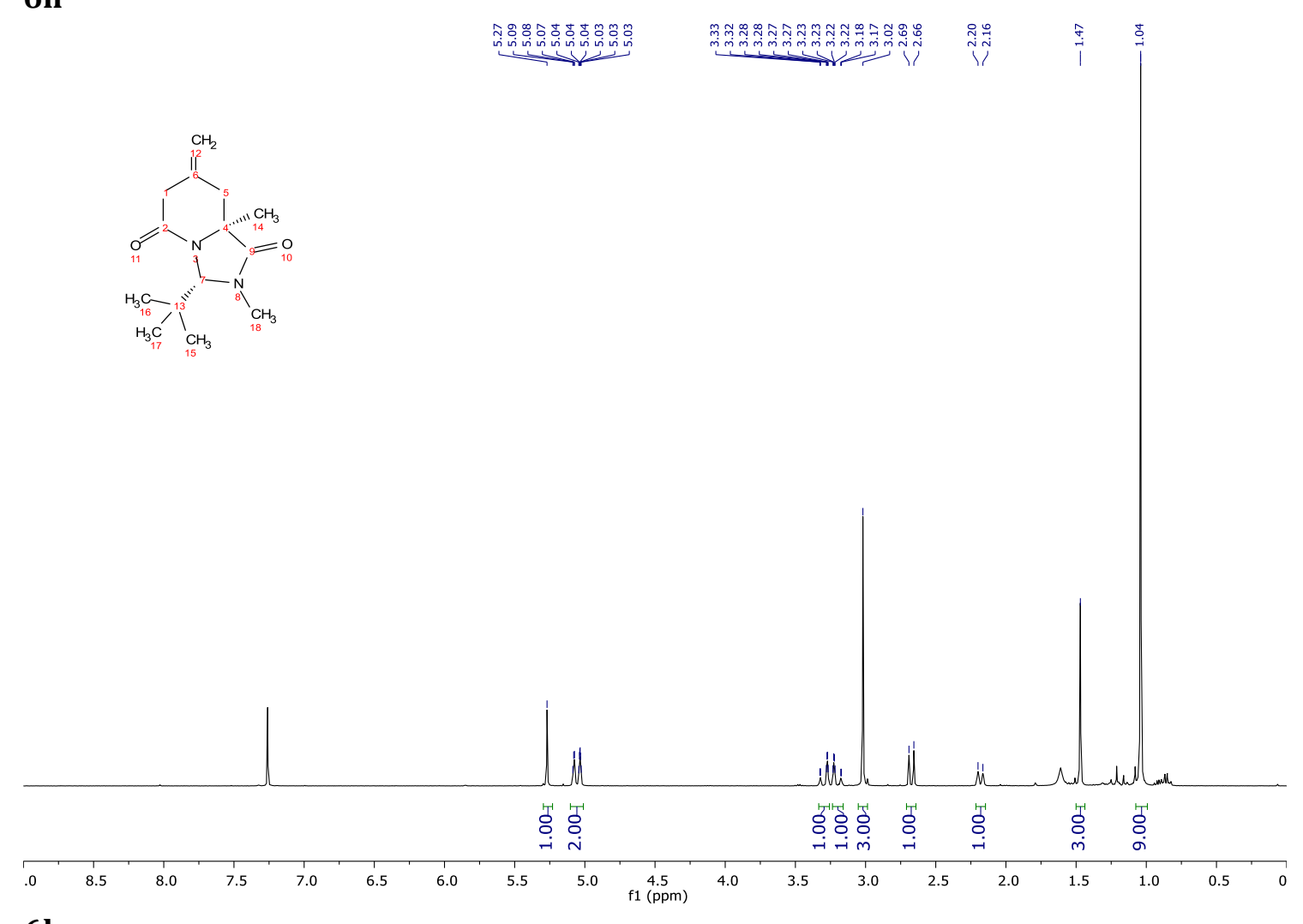

$6 h$

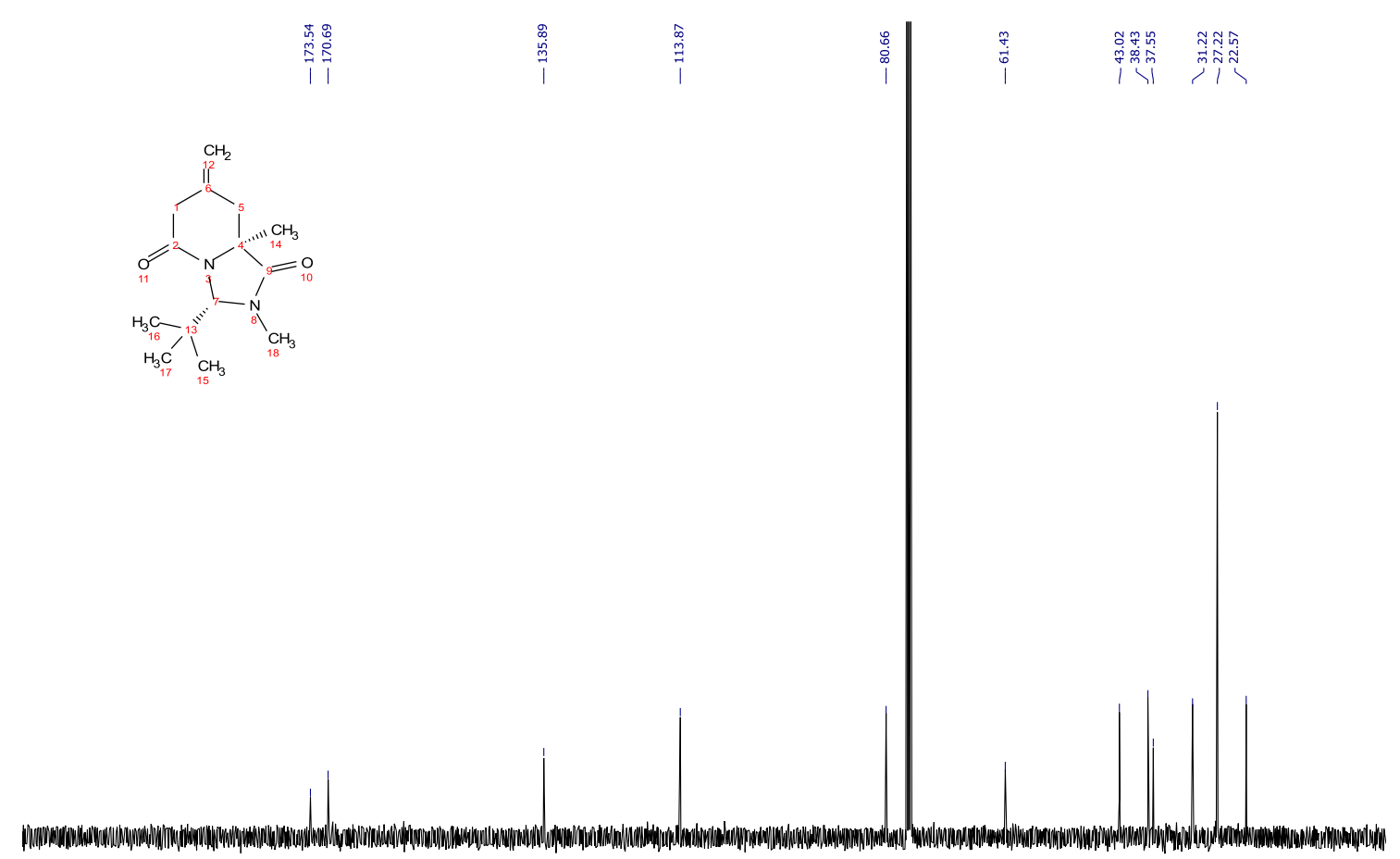

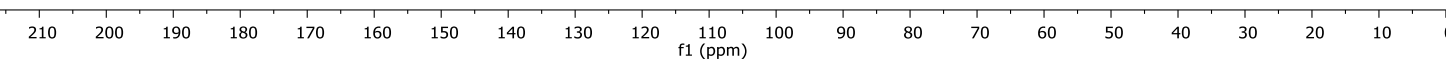


5

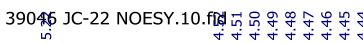

in

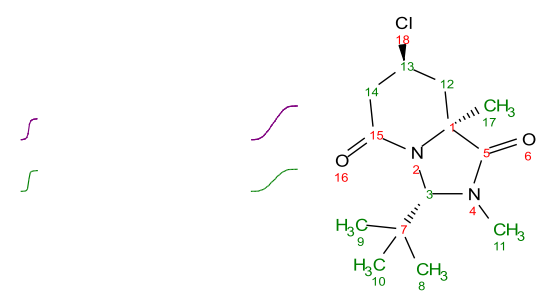

كمر

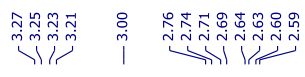

\begin{tabular}{|c|c|}
\hline$A(s)$ & $B(d t d)$ \\
5.22 & 4.48 \\
\hline
\end{tabular}

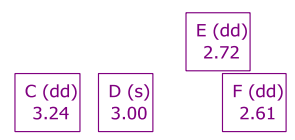

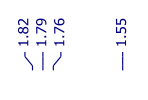

\begin{tabular}{l|l|}
$\mathrm{G}(\mathrm{t})$ & $\mathrm{H}(\mathrm{s})$ \\
1.79 & 1.55 \\
\hline
\end{tabular}

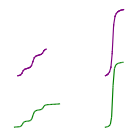

$-45000$

$-40000$

35000

30000

I (s) -25000 1.04
$3,9,10$

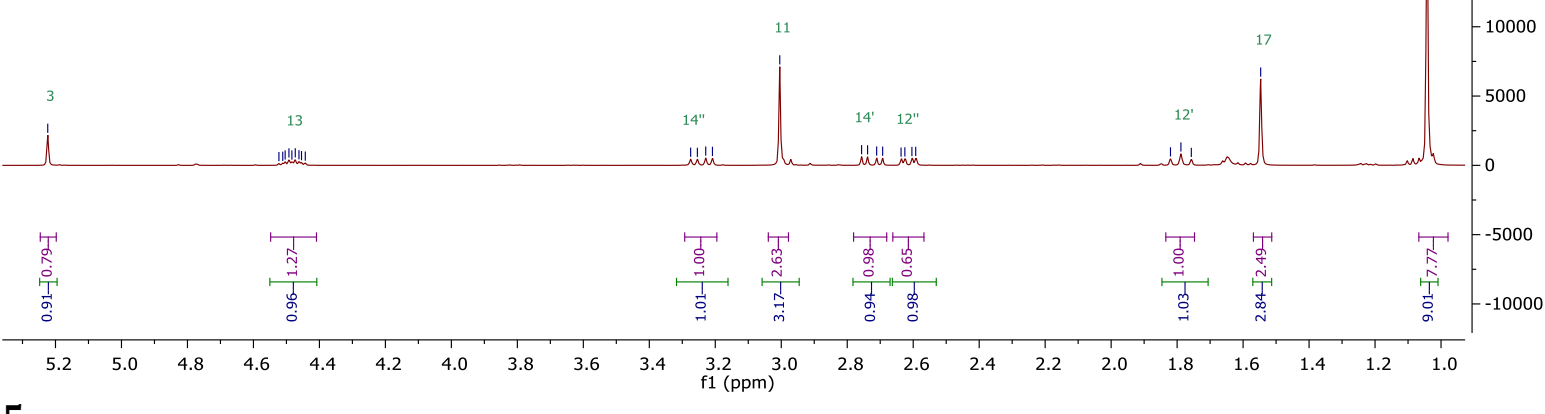

5

38765 JC 22 fr5.14.fid

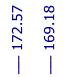

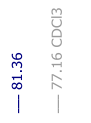

l $\quad$ l

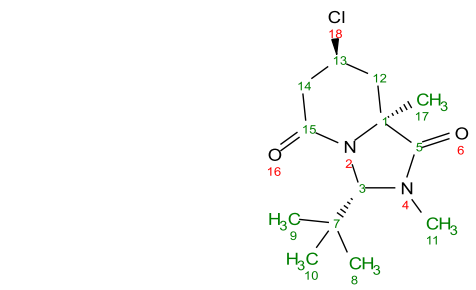

4500

$-4000$

$-3500$

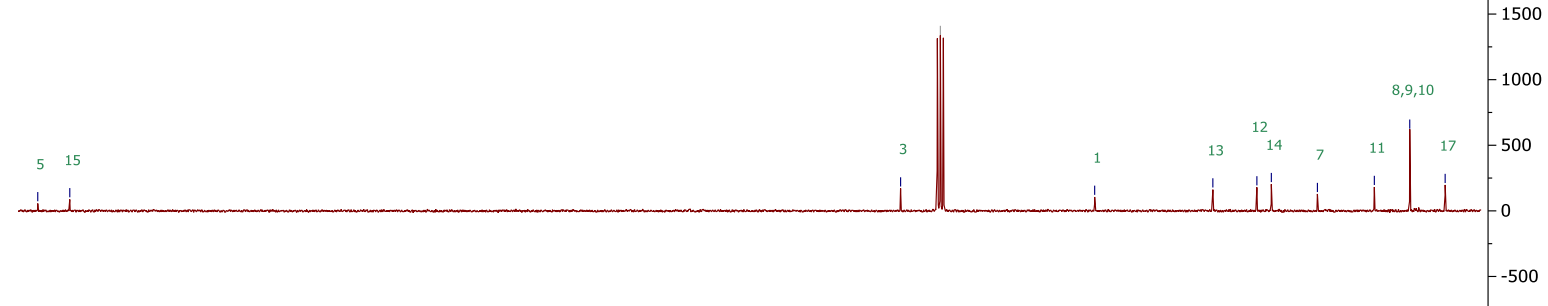

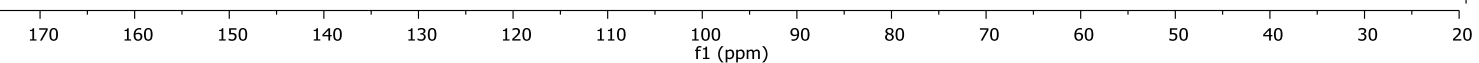




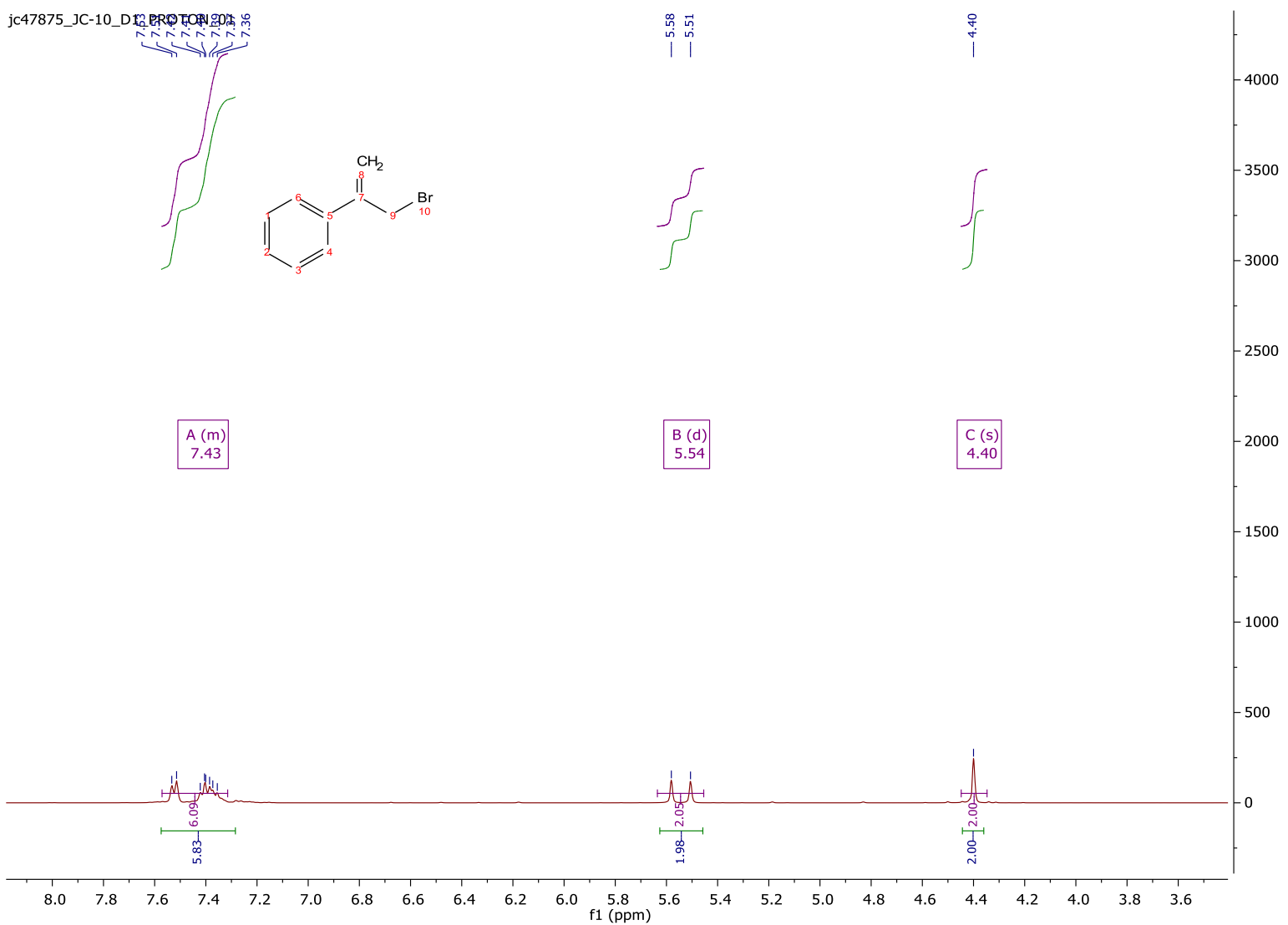


S4
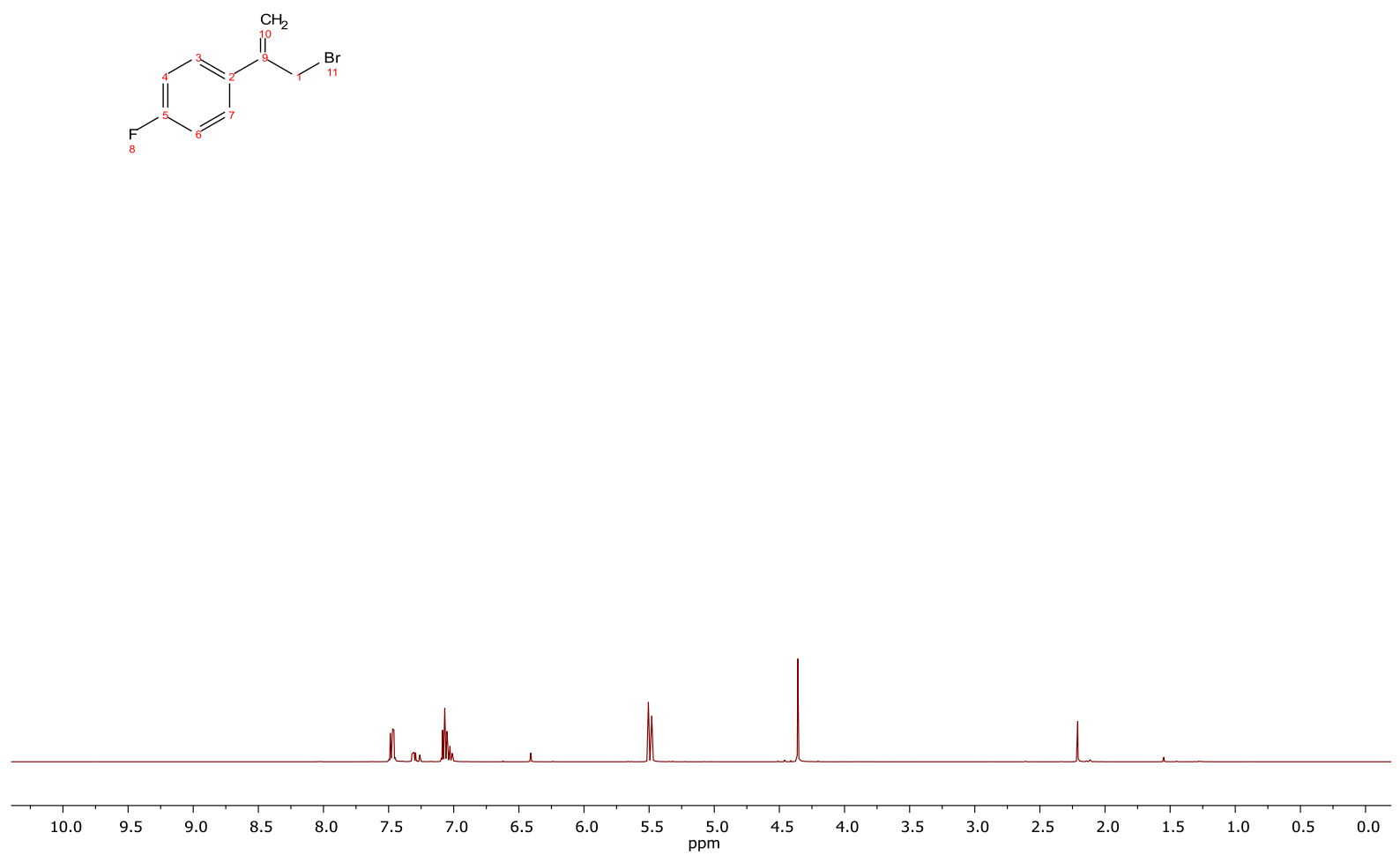

S4
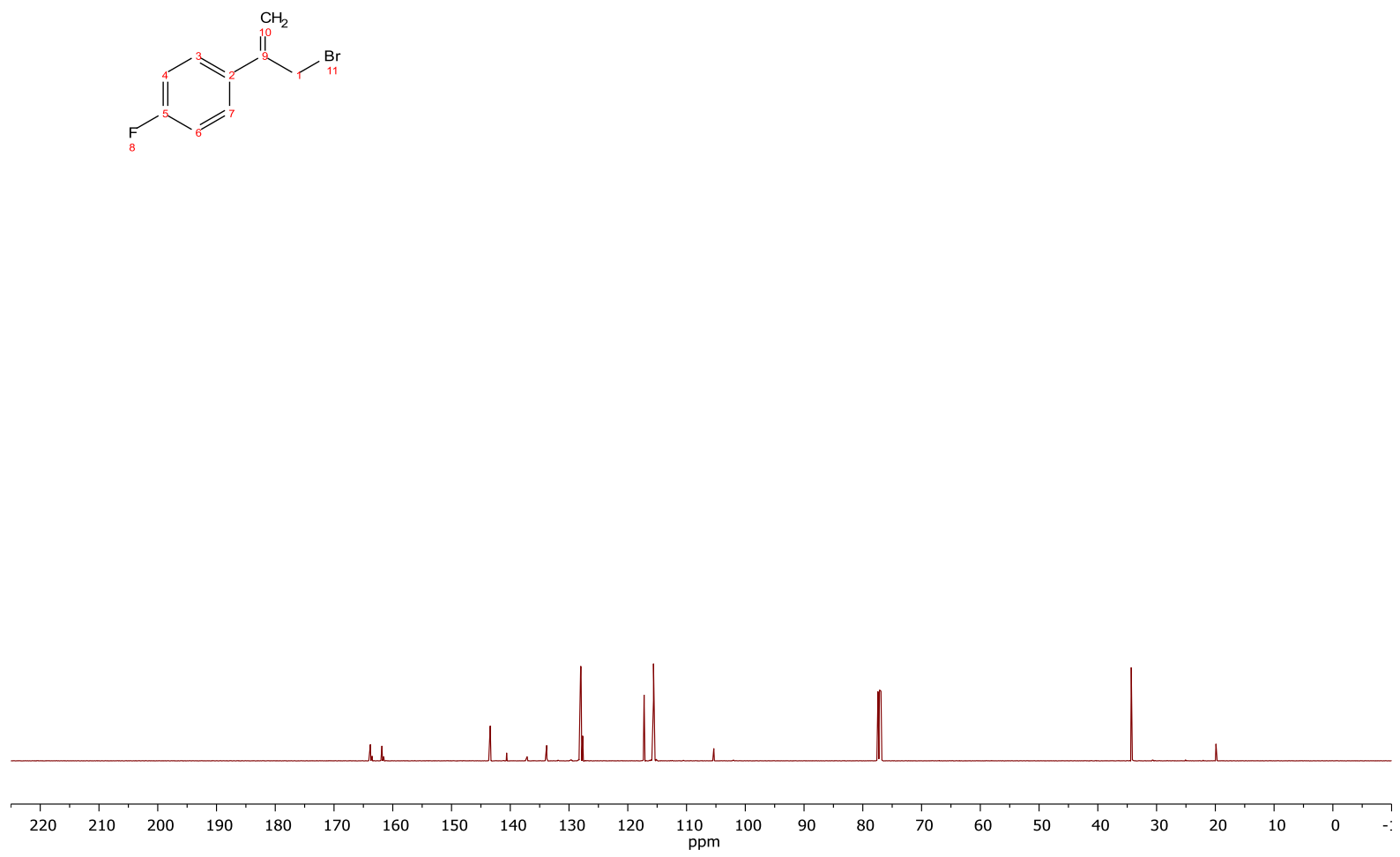

78 
S5
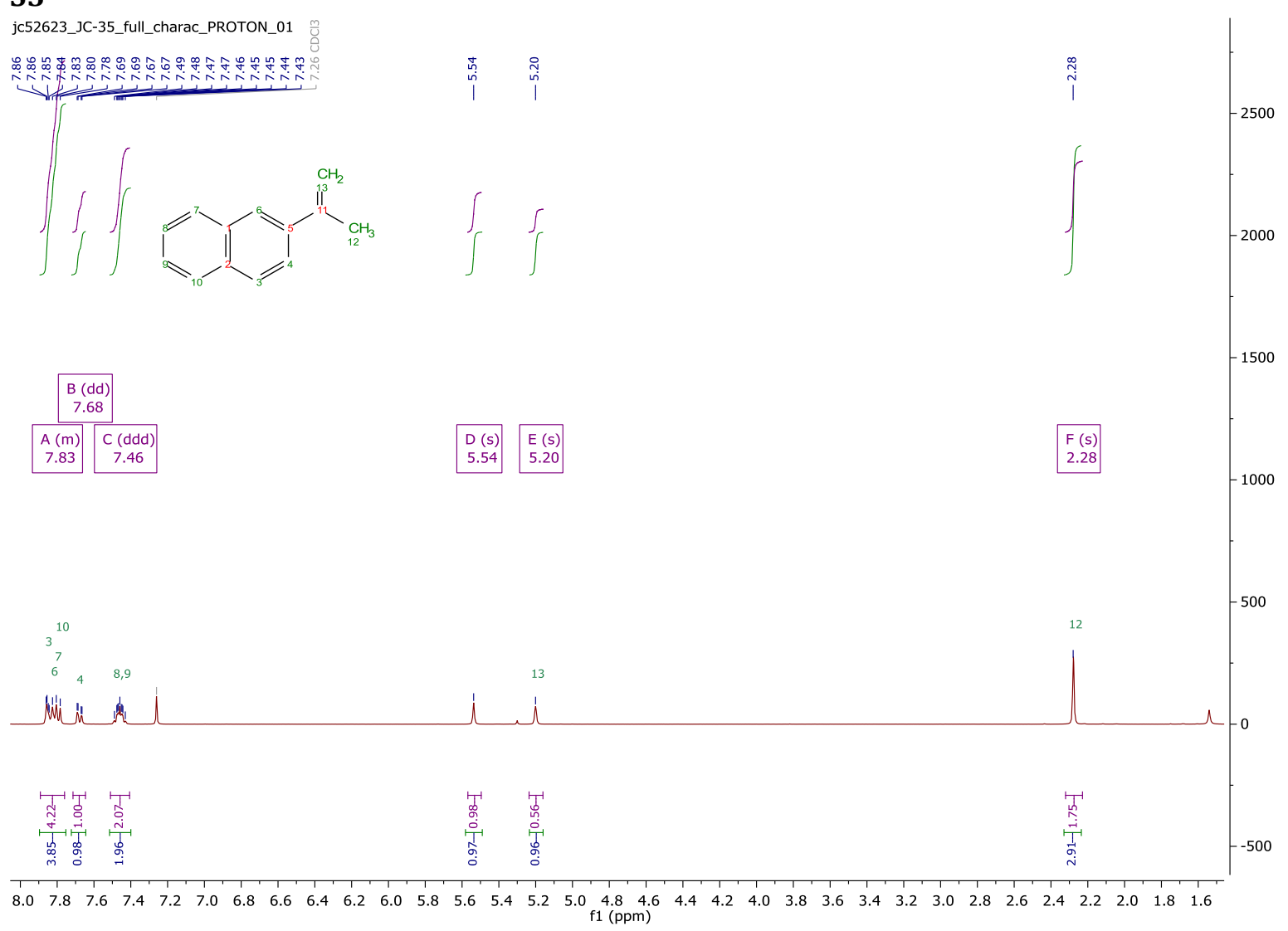

S5

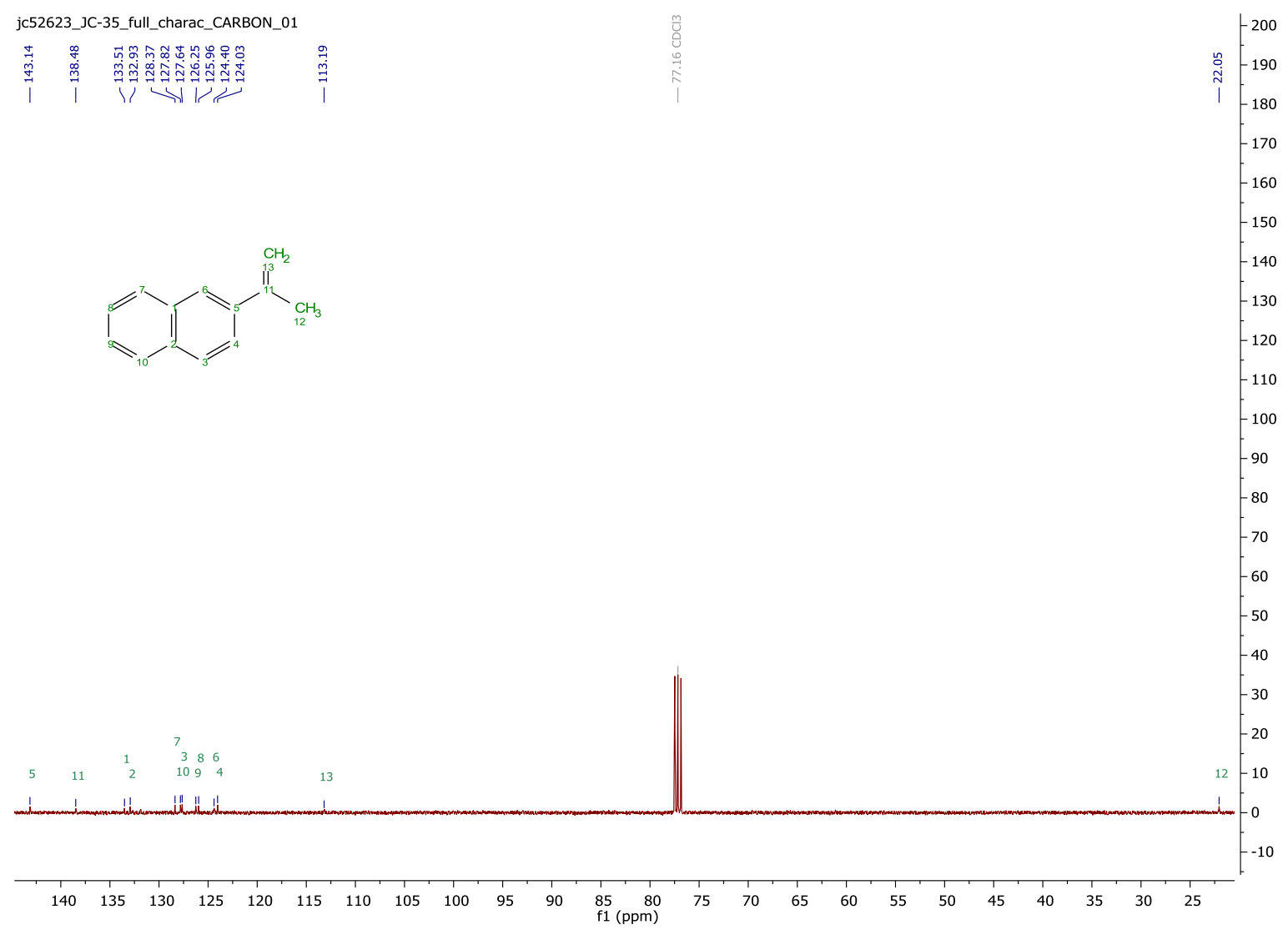

79 
S6

jcljc15571 JC-37 fr6-14

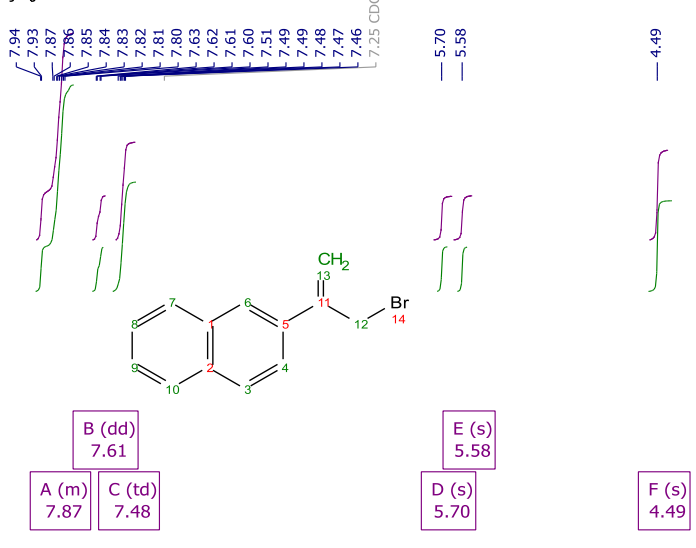

$-3.0$

(2)

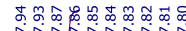

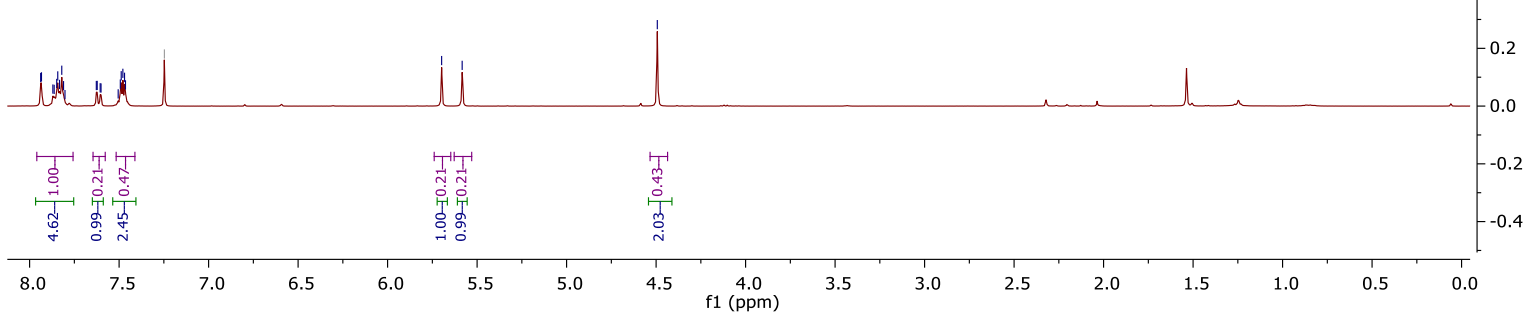




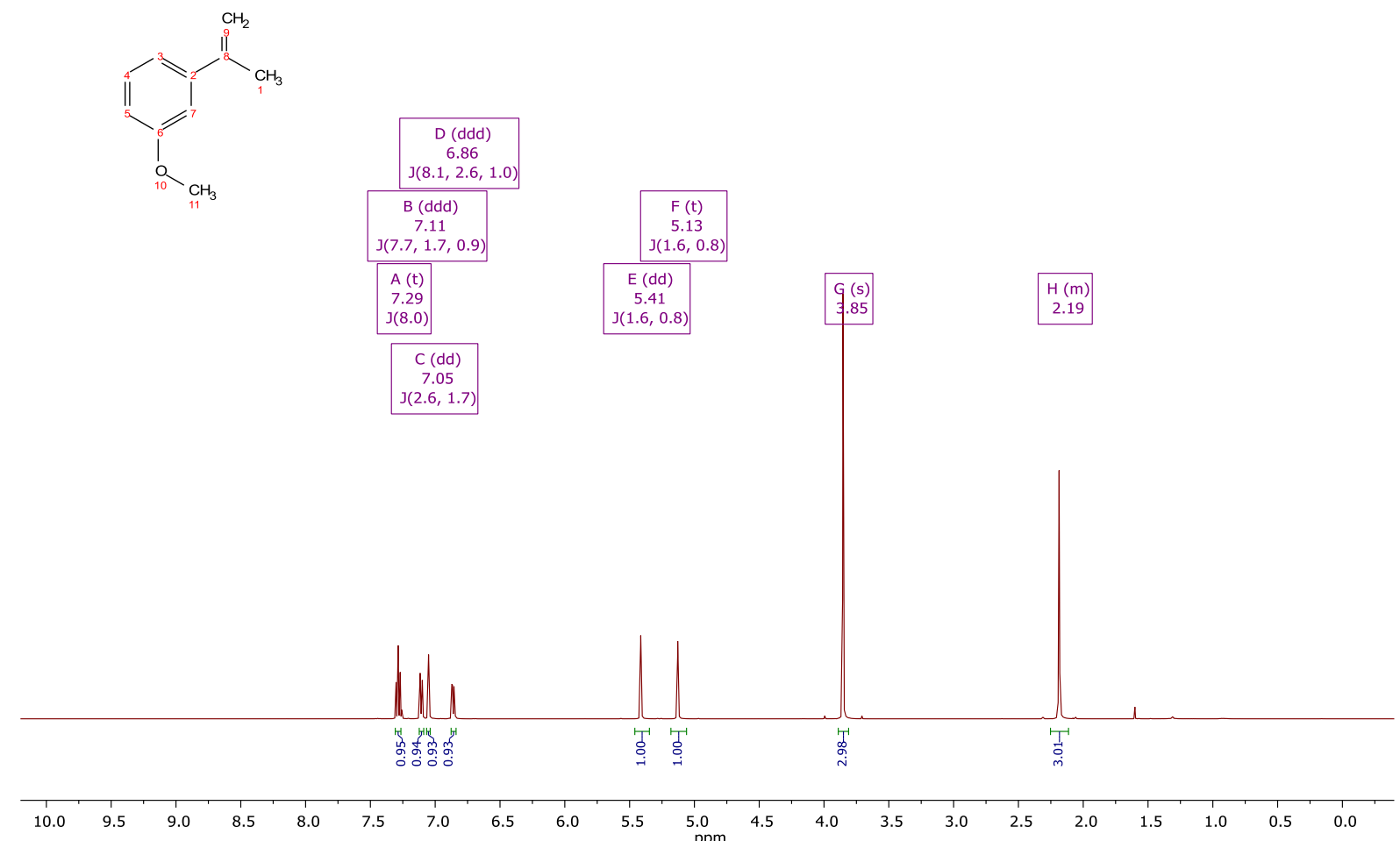

S7

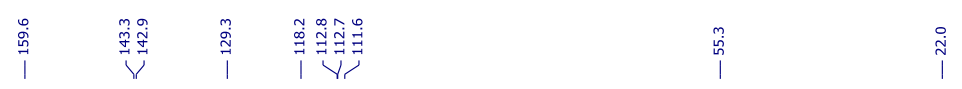<smiles>CC(C)c1cccc(OS)c1</smiles>

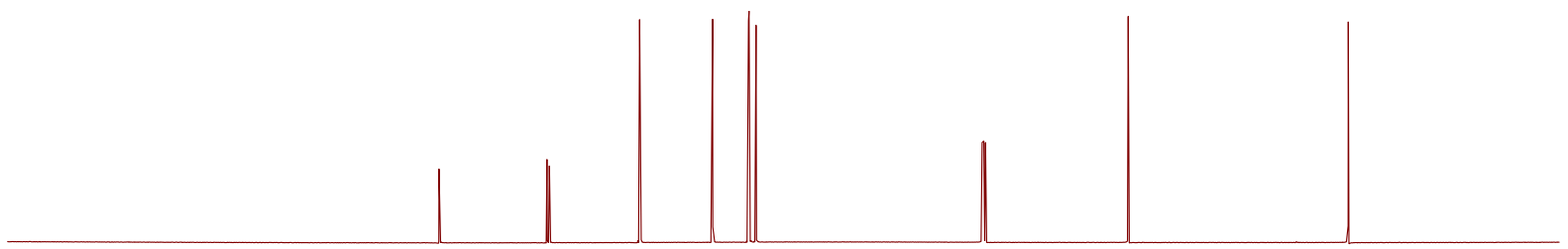

$\begin{array}{lllllllllllllllllllllllllllllllll}220 & 210 & 200 & 190 & 180 & 170 & 160 & 150 & 140 & 130 & 120 & 110 & 100 & 90 & 80 & 70 & 60 & 50 & 40 & 30 & 20 & 10 & 0 & -\end{array}$ 
S8

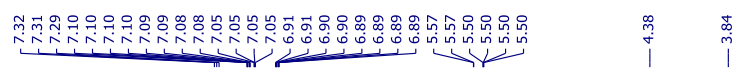<smiles>C=C(CBr)c1cccc(OC)c1</smiles>

\begin{tabular}{|c|}
\hline $\begin{array}{c}\text { (ddd) } \\
6.90 \\
\mathrm{~J}(8.2,2.6,0 . \\
\end{array}$ \\
\hline $\begin{array}{c}B(\text { ddd }) \\
7.09 \\
J(7.7,1.7,0.9)\end{array}$ \\
\hline $\begin{array}{c}A(t) \\
7.31 \\
J(8.0)\end{array}$ \\
\hline $\begin{array}{c}\mathrm{C}(\mathrm{dd}) \\
7.05 \\
\mathrm{~J}(2.6,1.7)\end{array}$ \\
\hline
\end{tabular}

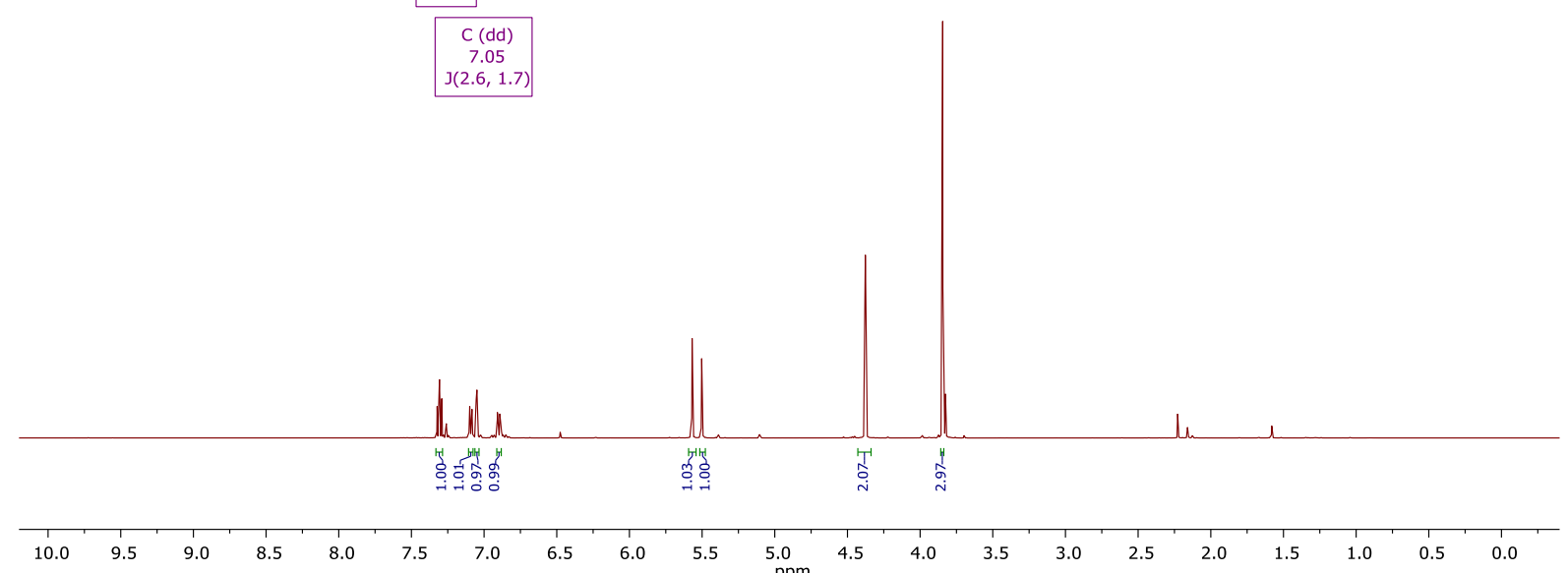

S8

l li<smiles>C=C(CBr)c1cccc(OC)c1</smiles>

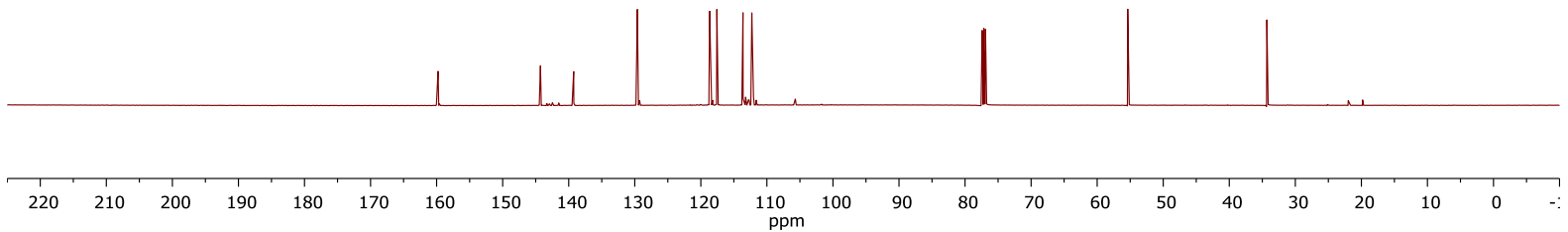


S9

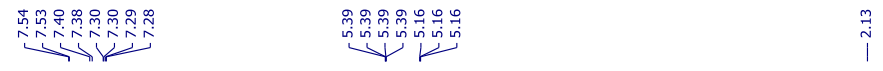<smiles>C=C(C)c1ccc(Cl)c(Cl)c1</smiles>

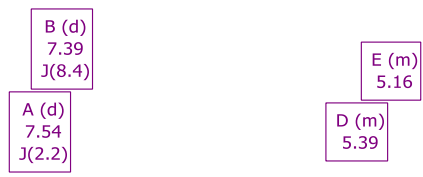

$F(s)$
2.13

C (dd)

$\mathrm{J}(8.4,2.1)$

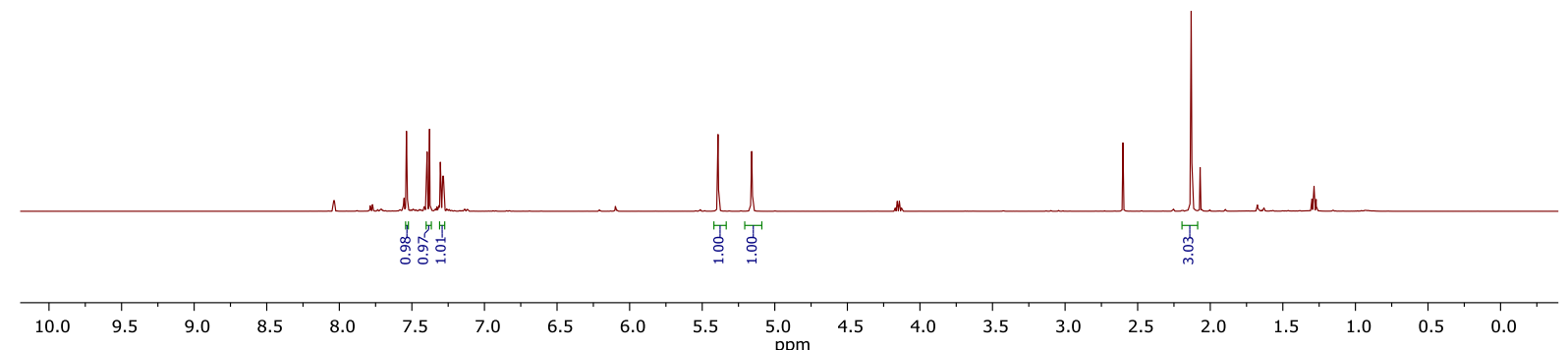

S9

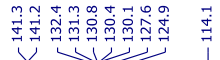<smiles>CC(C)c1ccc(Cl)c(Cl)c1</smiles>

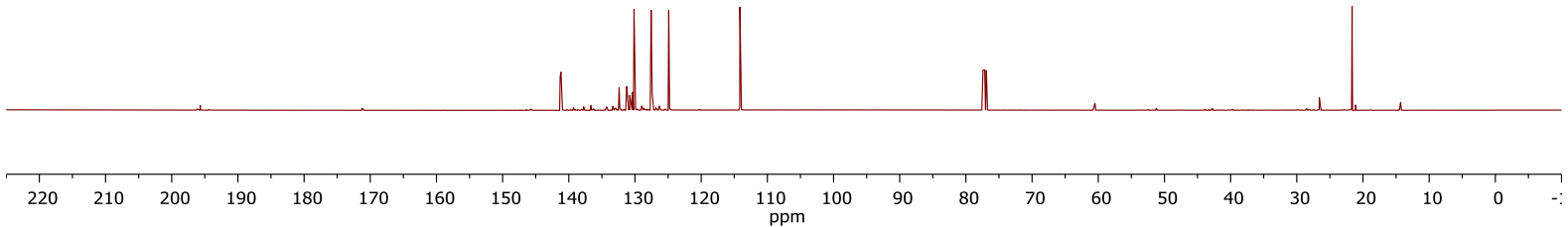


S10

$\underbrace{.0}$

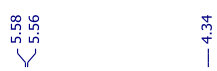

(2)
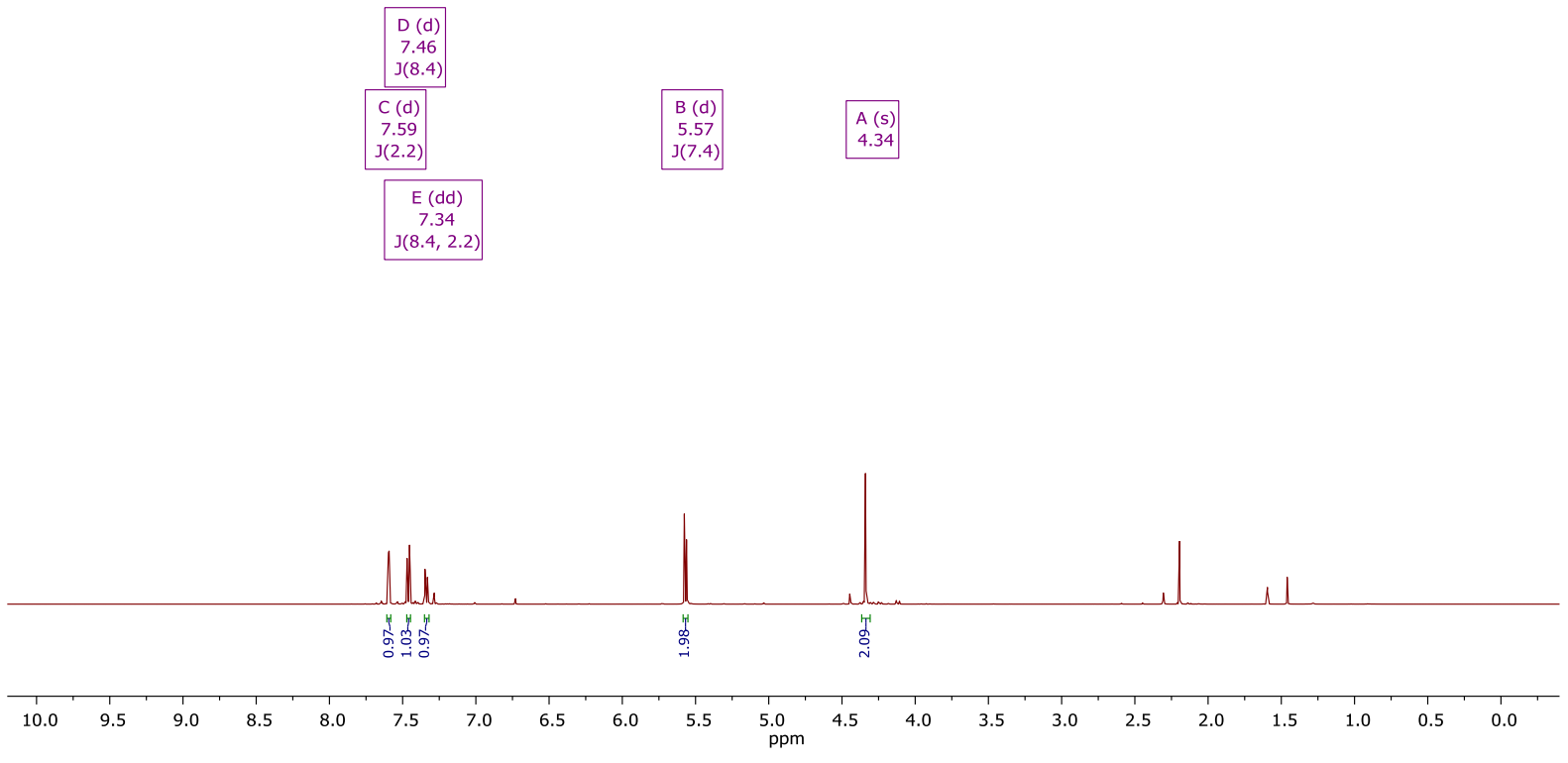

S10

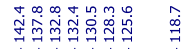
$\stackrel{\substack{m \\ m}}{i}$<smiles>C#CCc1ccc(Cl)c(Cl)c1</smiles>

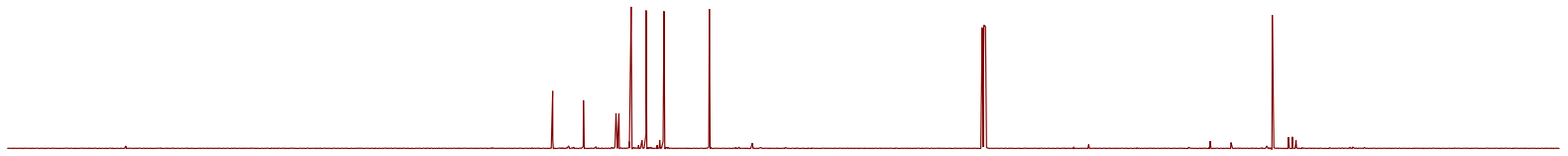

$\begin{array}{lllllllllllllllllllllllllllllllllllll}220 & 210 & 200 & 190 & 180 & 170 & 160 & 150 & 140 & 130 & 120 & 110 & 100 & 90 & 80 & 70 & 60 & 50 & 40 & 30 & 20 & 10 & 0 & -\therefore\end{array}$ 
S11

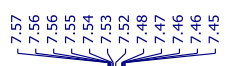
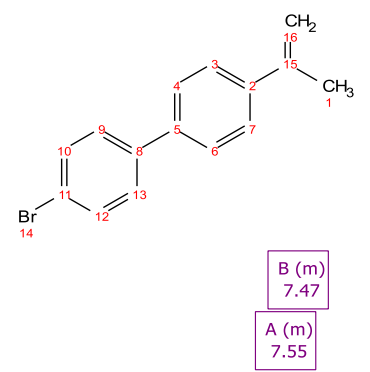
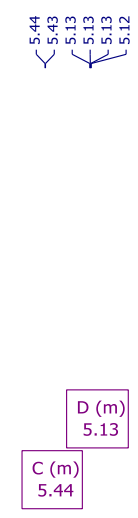

$\stackrel{9}{i}$

\begin{tabular}{|l|}
\hline$E(s)$ \\
2.19
\end{tabular}
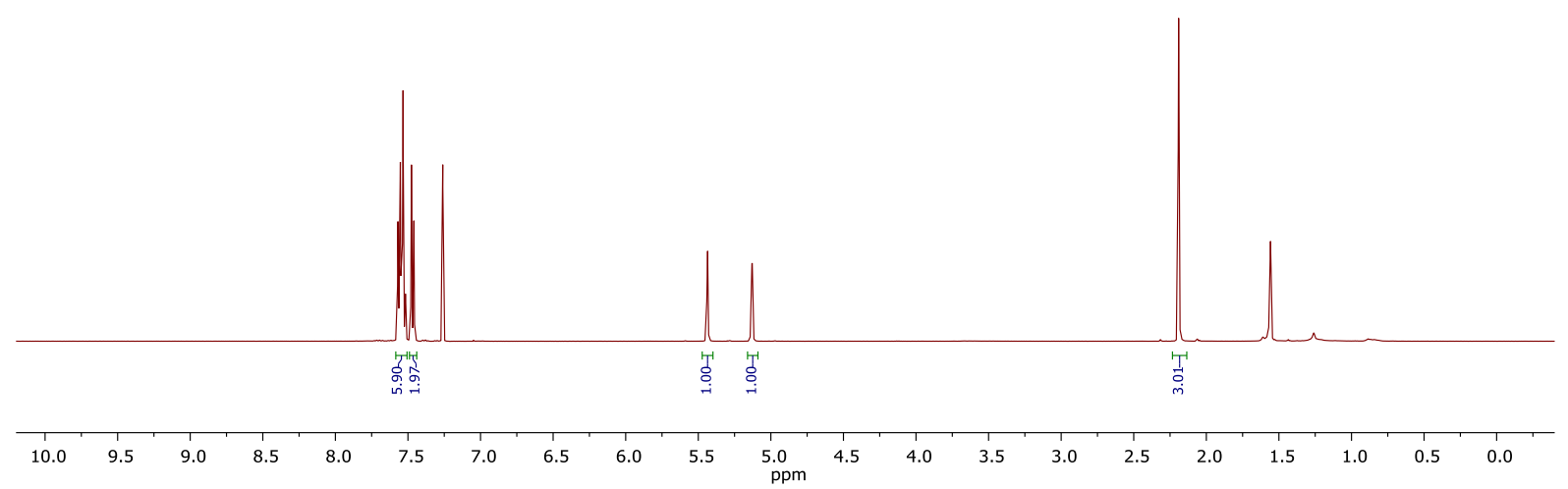

S11

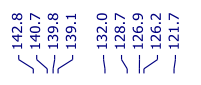

$\stackrel{\vec{i}}{1}$
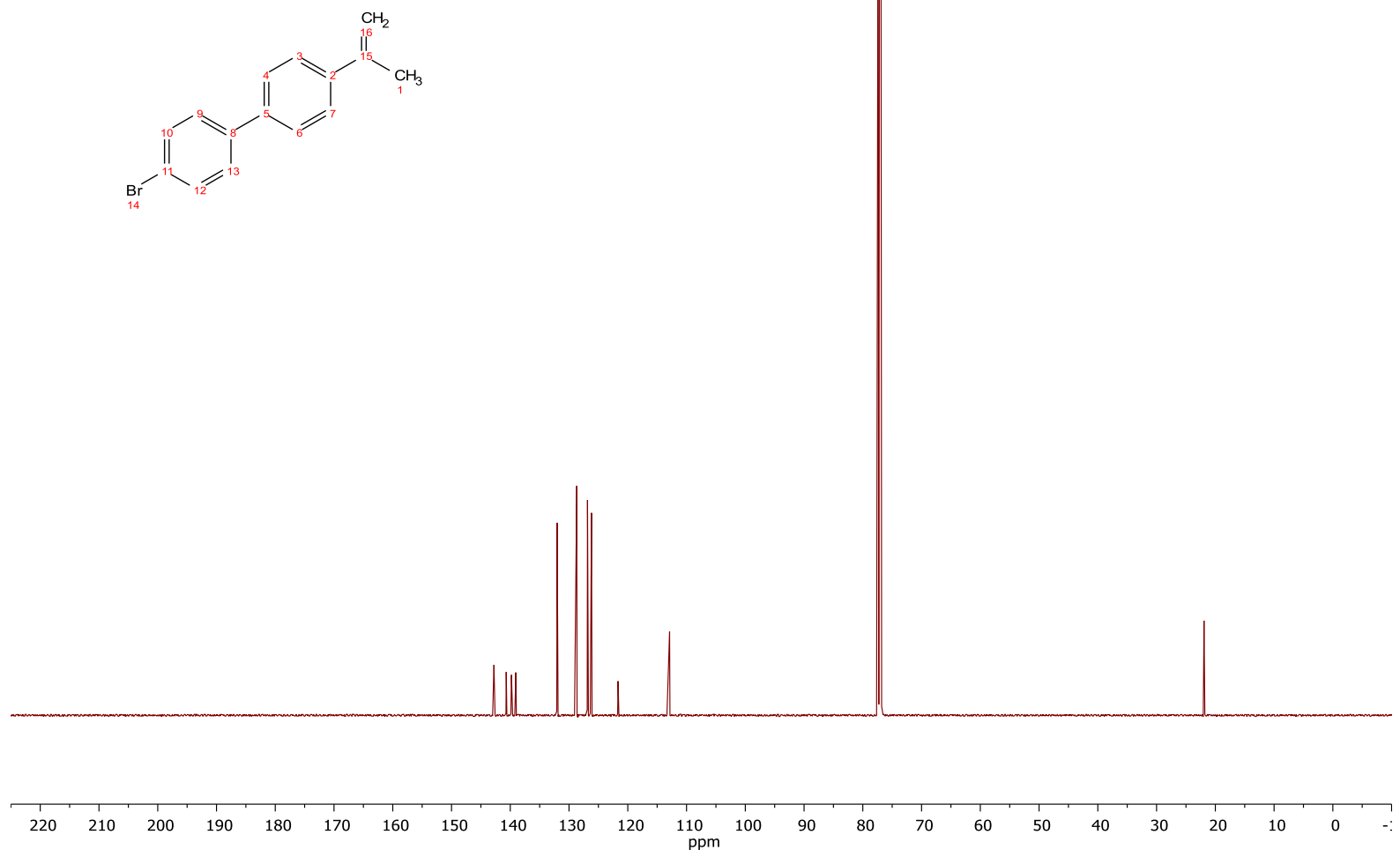

85 
S12

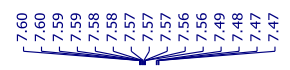

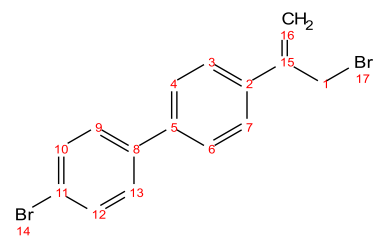

B (m)
7.58

A (m)
7.48

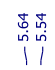

$C(d)$
5.59
$J(48.5)$ $\stackrel{9}{+}$

\begin{tabular}{|l|}
\hline $\mathrm{D}(\mathrm{s})$ \\
4.43 \\
\hline
\end{tabular}

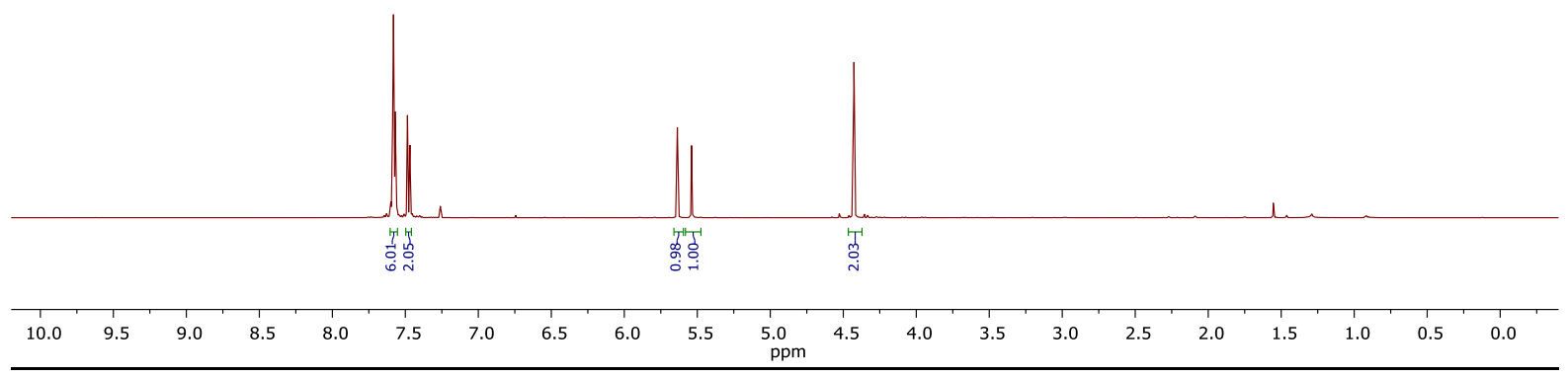

S12

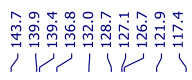

$\stackrel{7}{+}$
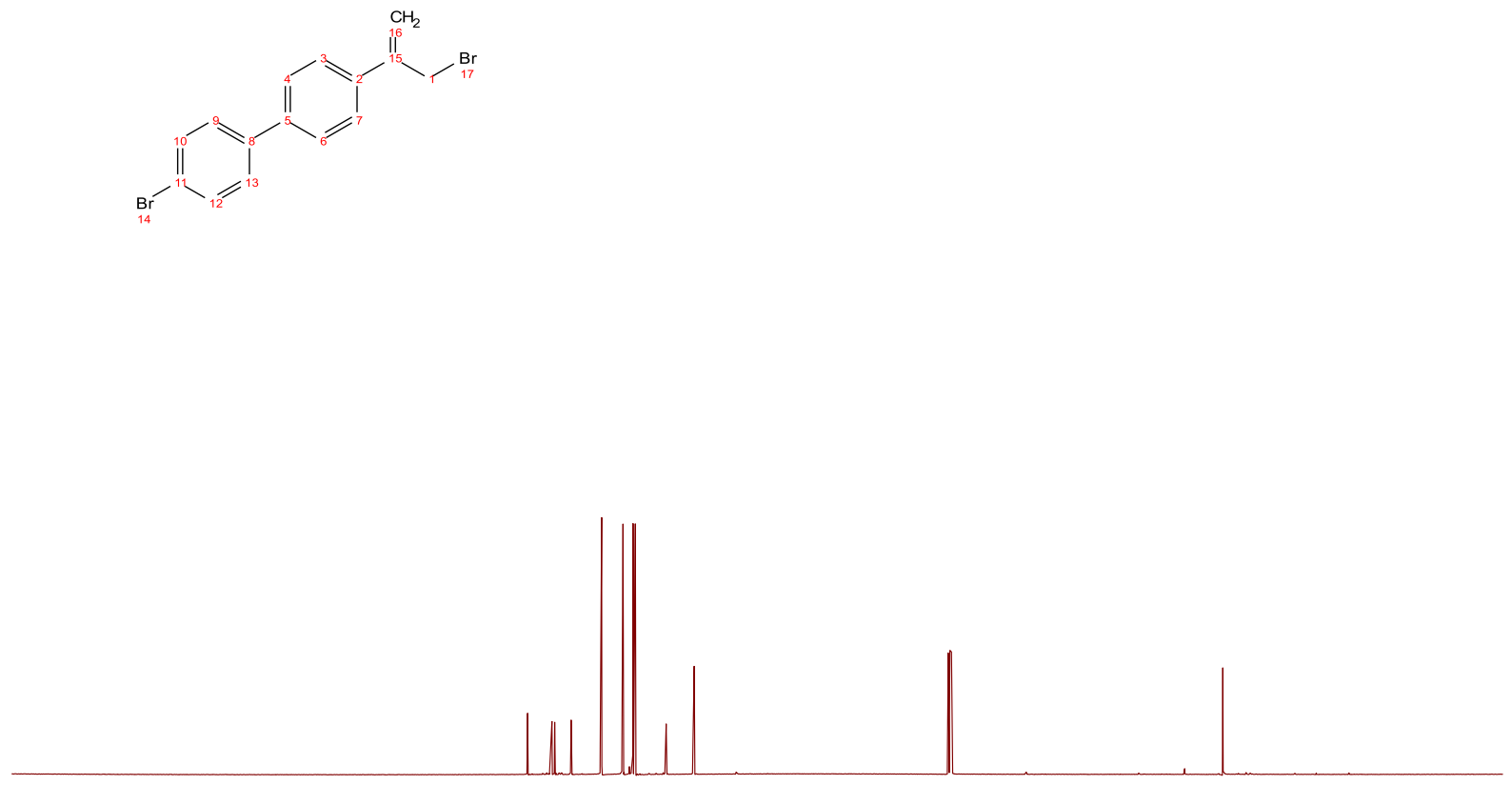

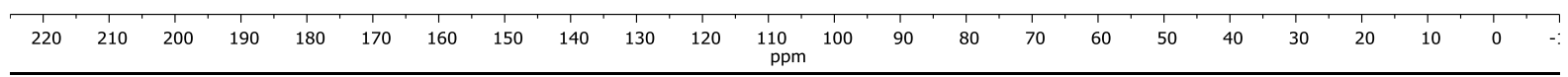

86 
S13

jc47003_jc_jc06crude_PROTON_01

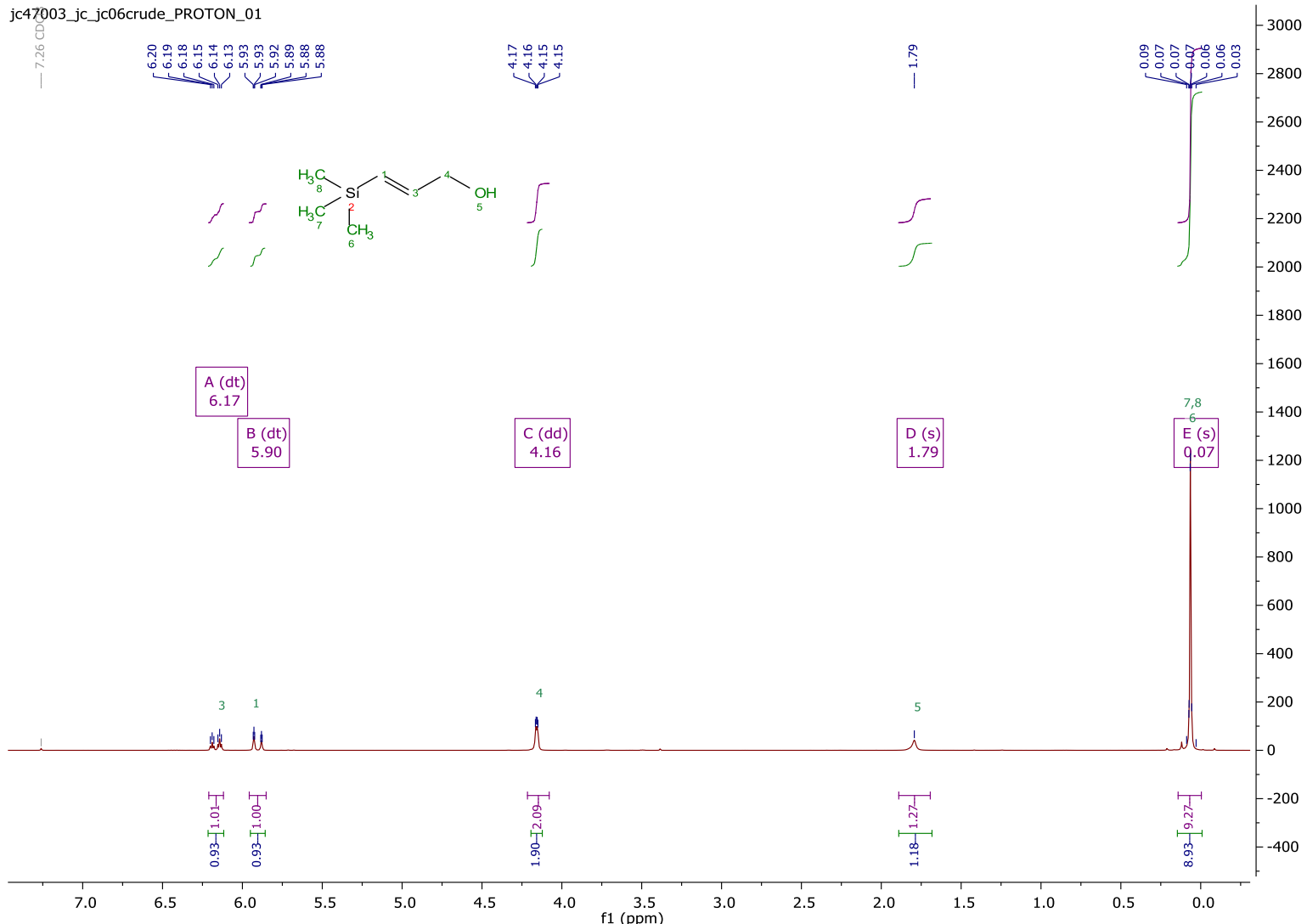


S14

38778 JC-23 charac.10.fid

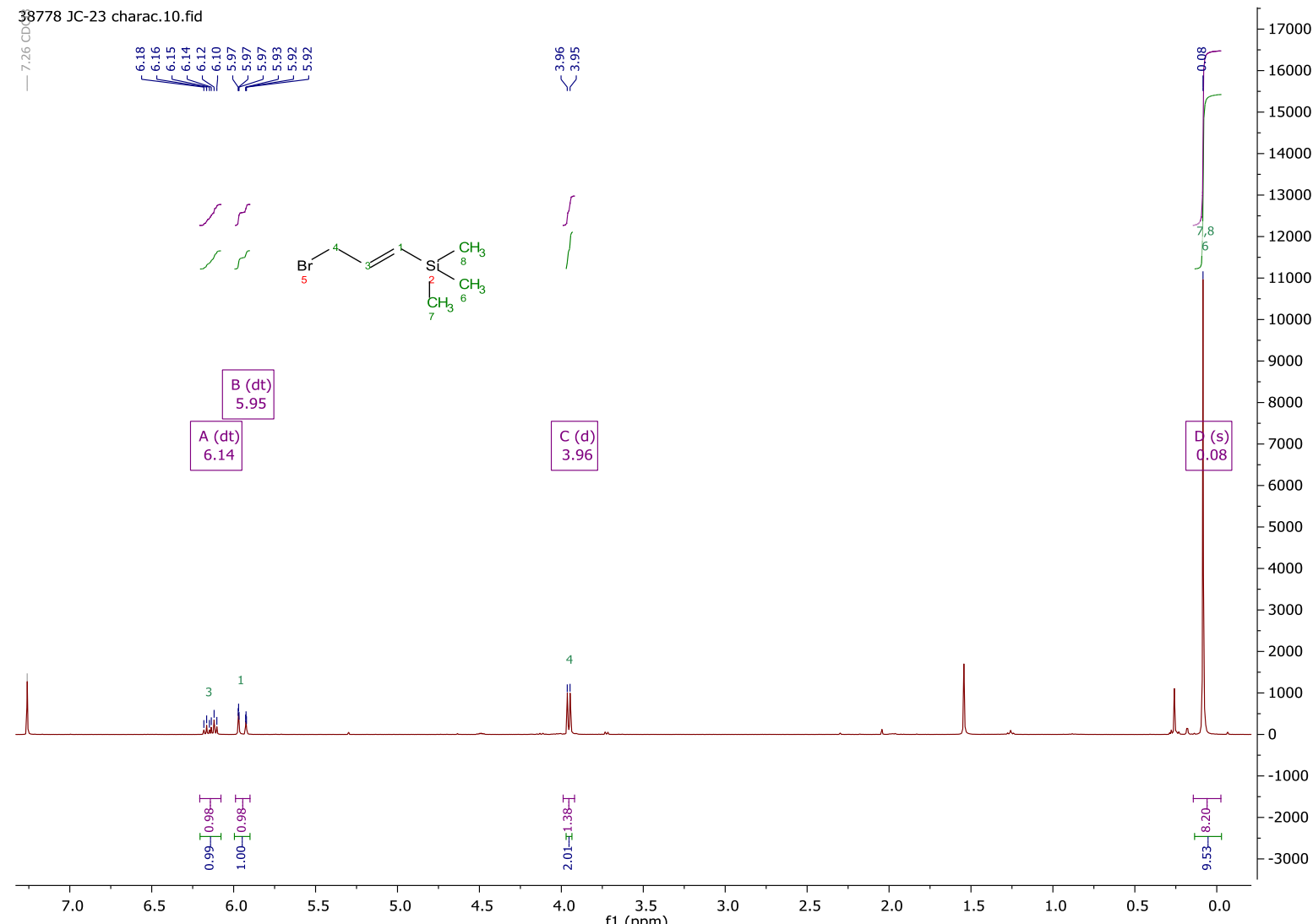

S14

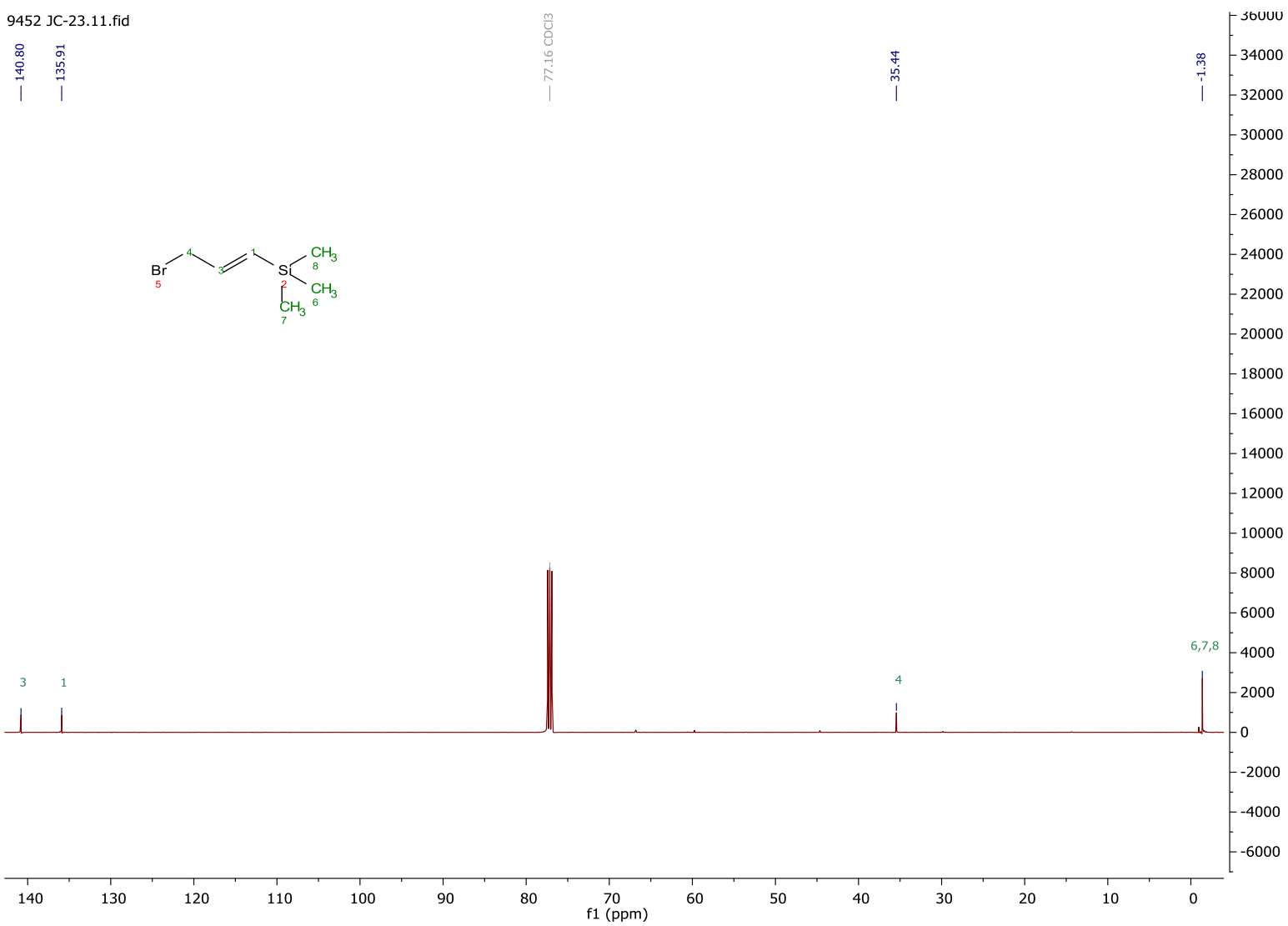




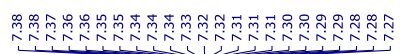

F

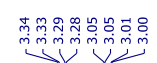

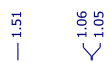

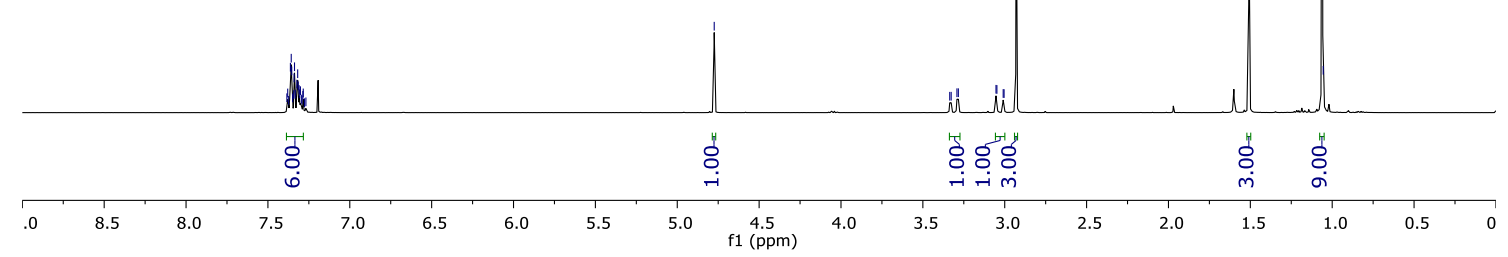

$E-7 \mathbf{i}$
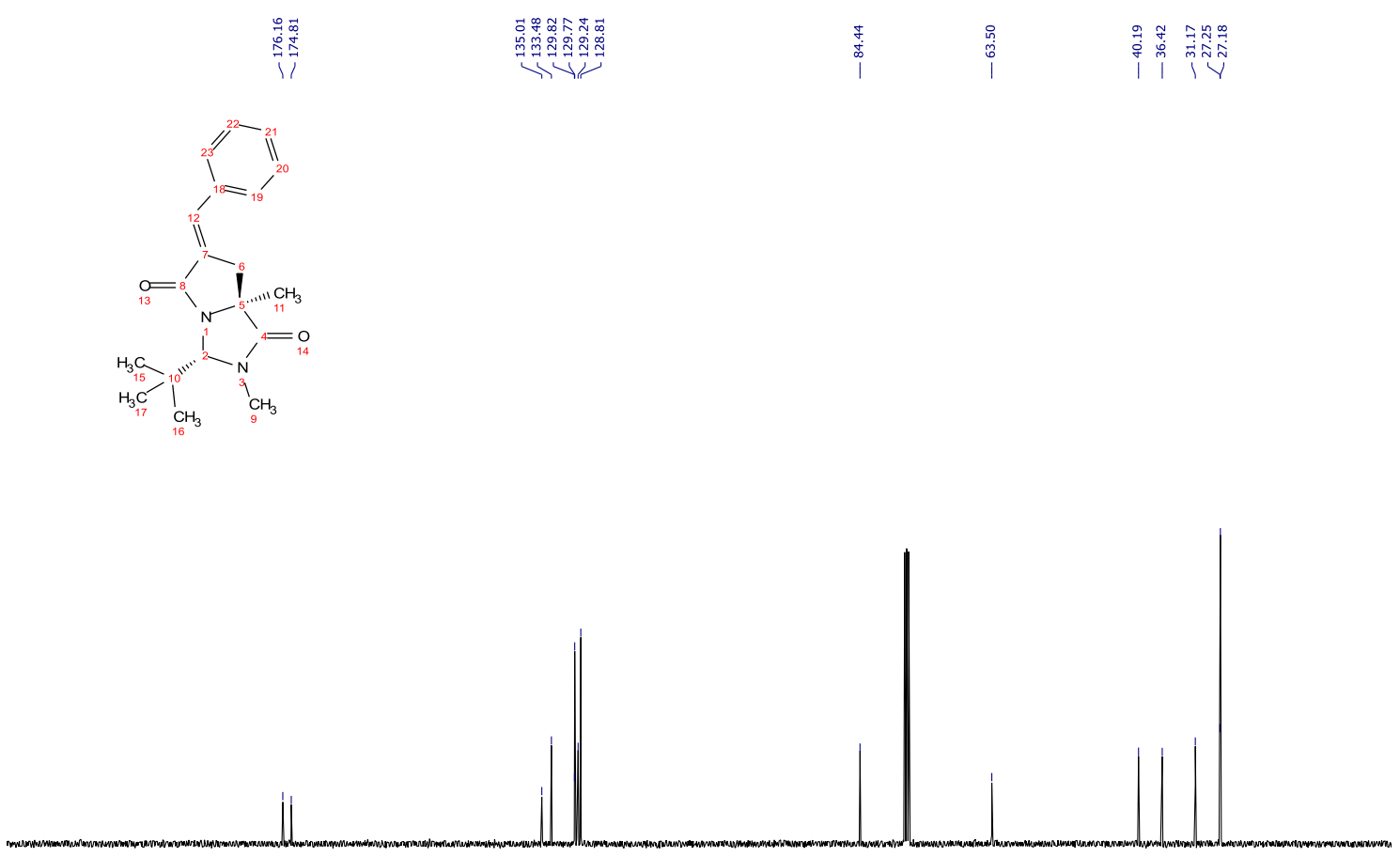

$\begin{array}{lllllllllllllllllllllll} & 210 & 200 & 190 & 180 & 170 & 160 & 150 & 140 & 130 & 120 & 110 & 100 & 90 & 80 & 70 & 60 & 50 & 40 & 30 & 20 & 10 & 1\end{array}$ 

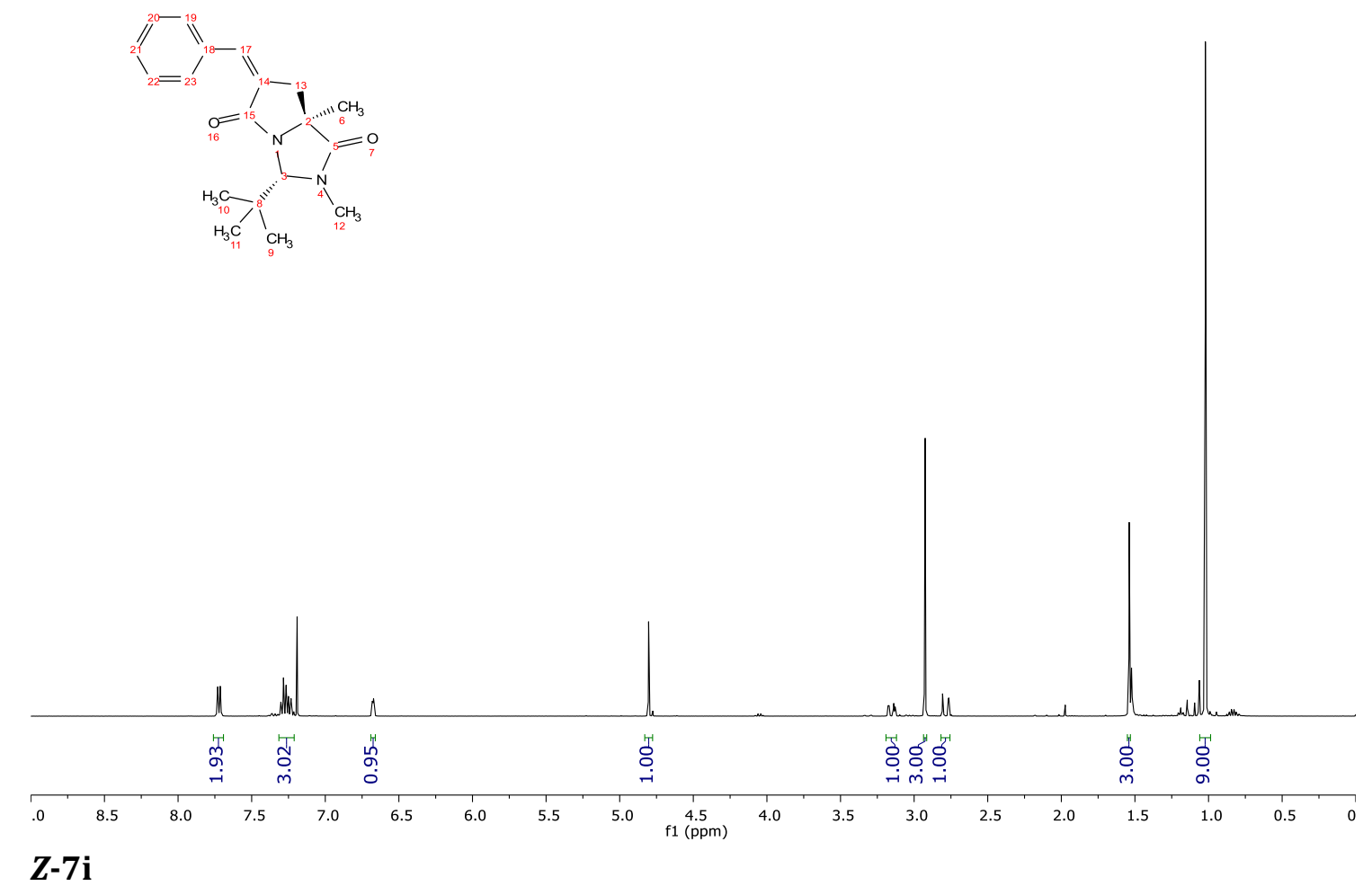

Z-7i

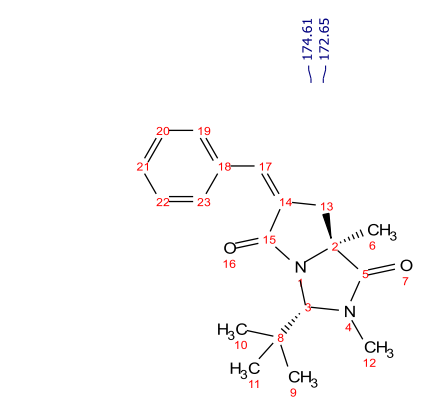

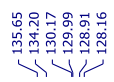

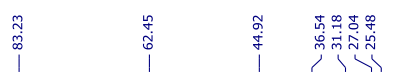
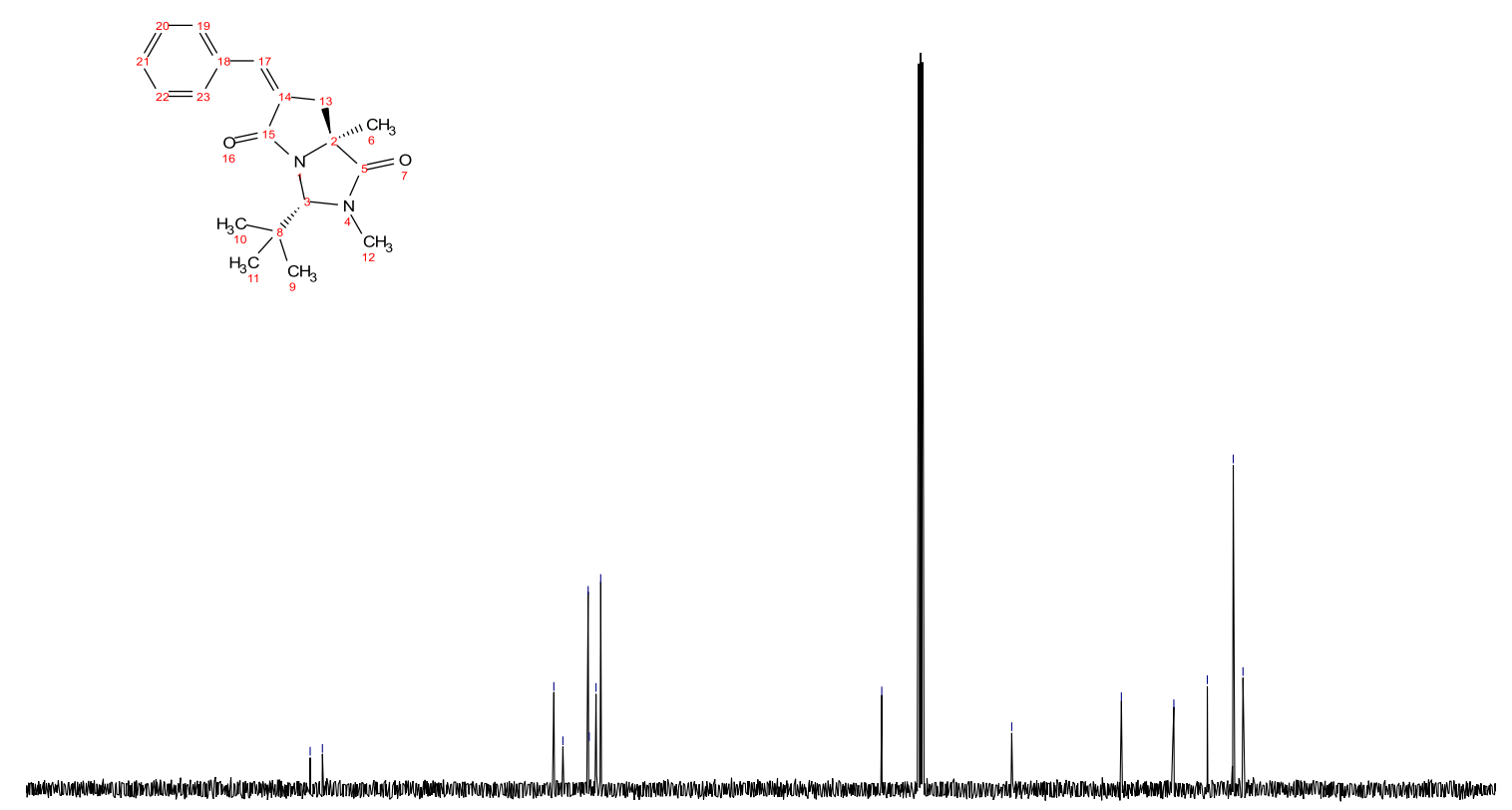

$\begin{array}{llllllllllll}210 & 200 & 190 & 180 & 170 & 160 & 150 & 140 & 130 & 120 & 110 & 100 \\ \mathrm{f} 1(\mathrm{ppm})\end{array}$ 
7j

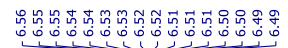

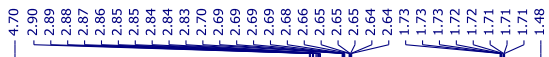

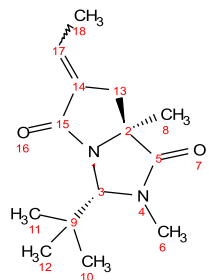

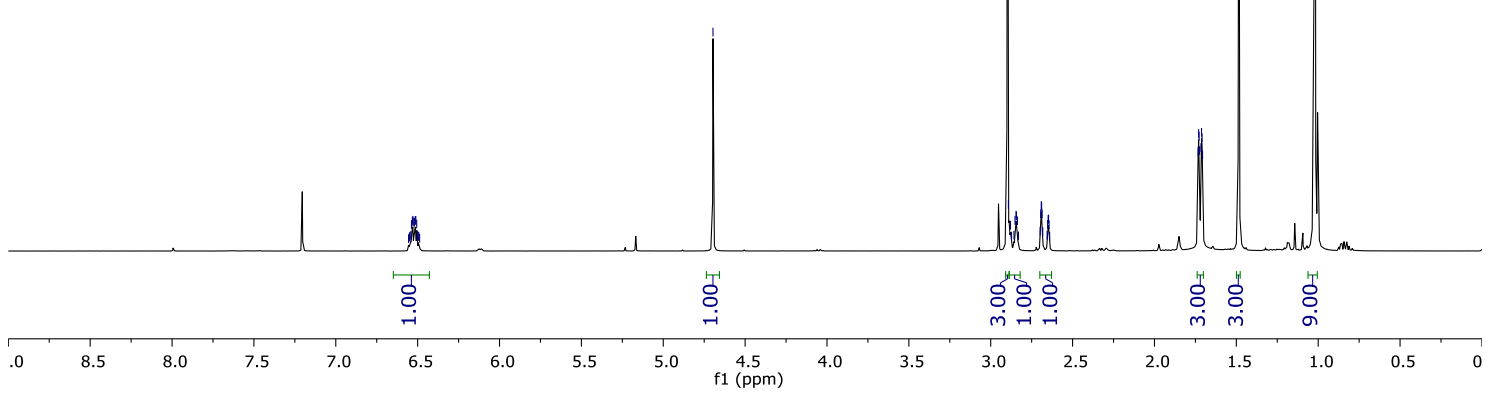

$7 \mathbf{j}$
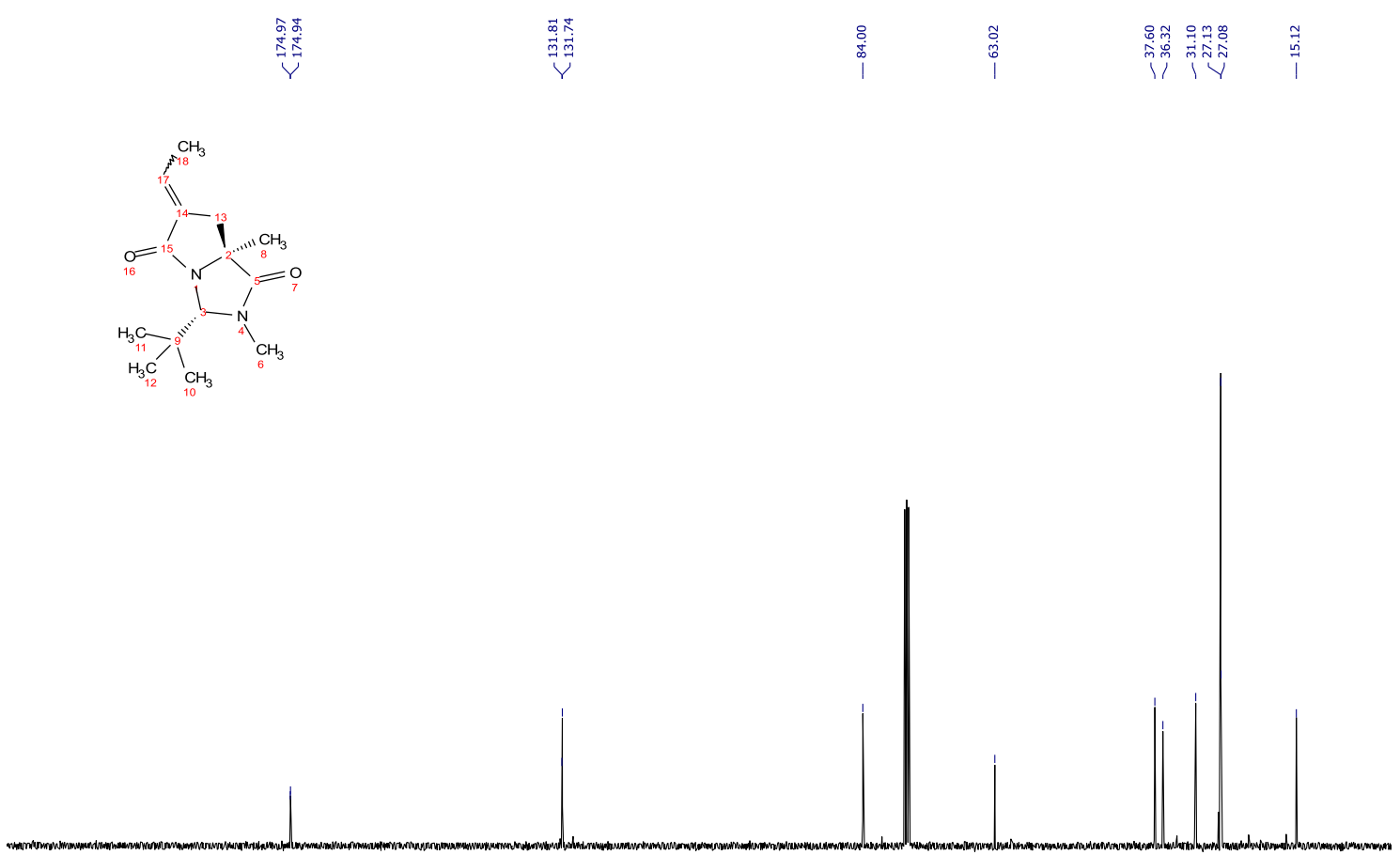

$\begin{array}{lllllllllllllllllllll}210 & 200 & 190 & 180 & 170 & 160 & 150 & 140 & 130 & 120 & 110 & 100 & 90 & 80 & 70 & 60 & 50 & 40 & 30 & 20 & 10\end{array}$ 
8j

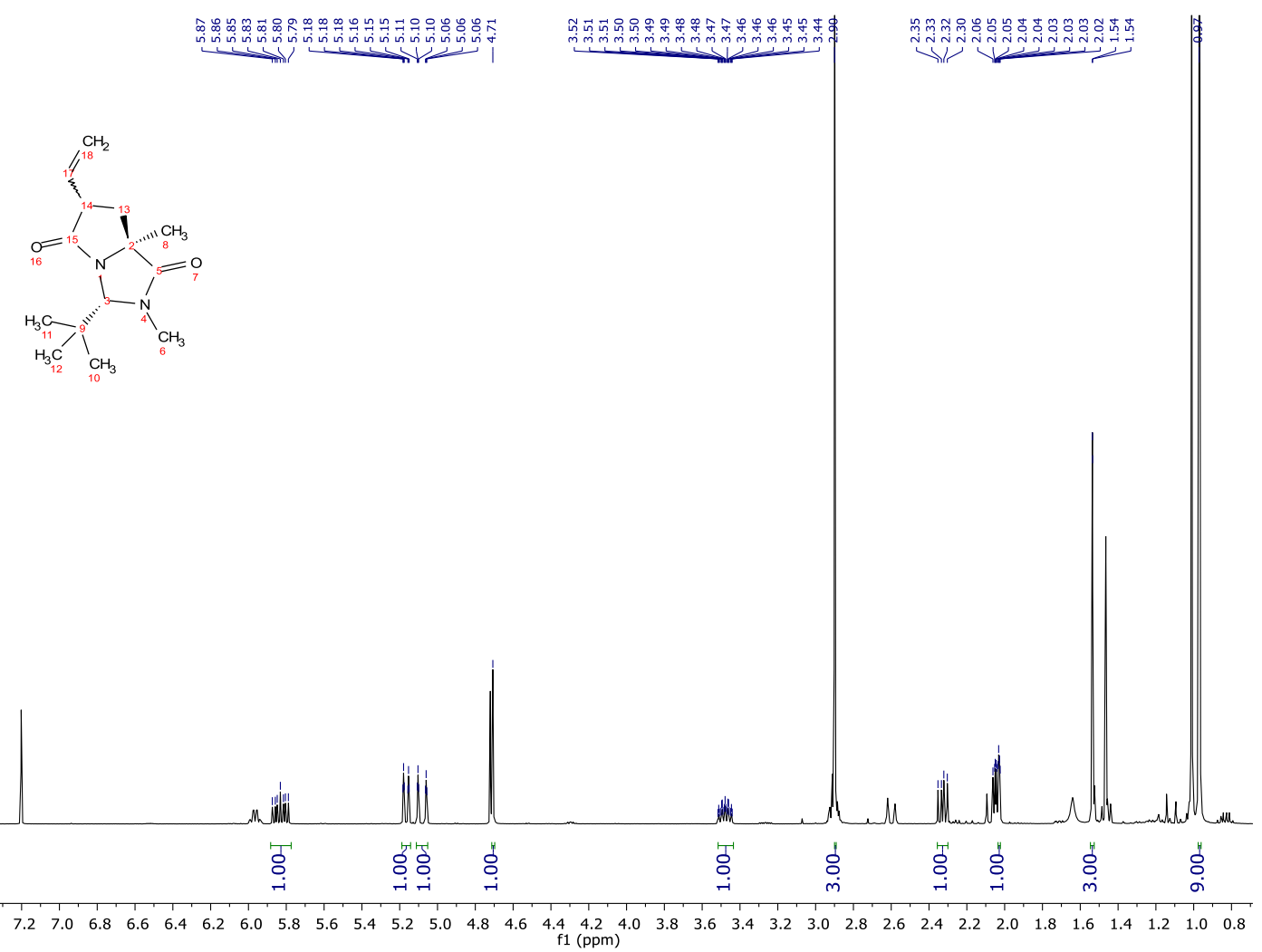

8j

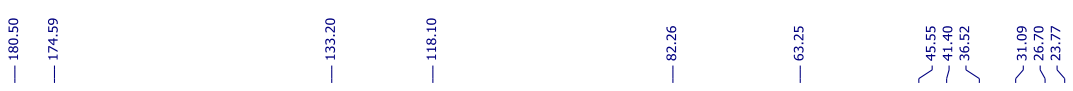<smiles>C=CN1NC(C)(C)C(=O)N(CC)C1C(C)(C)C</smiles>

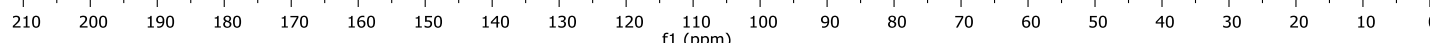


9a

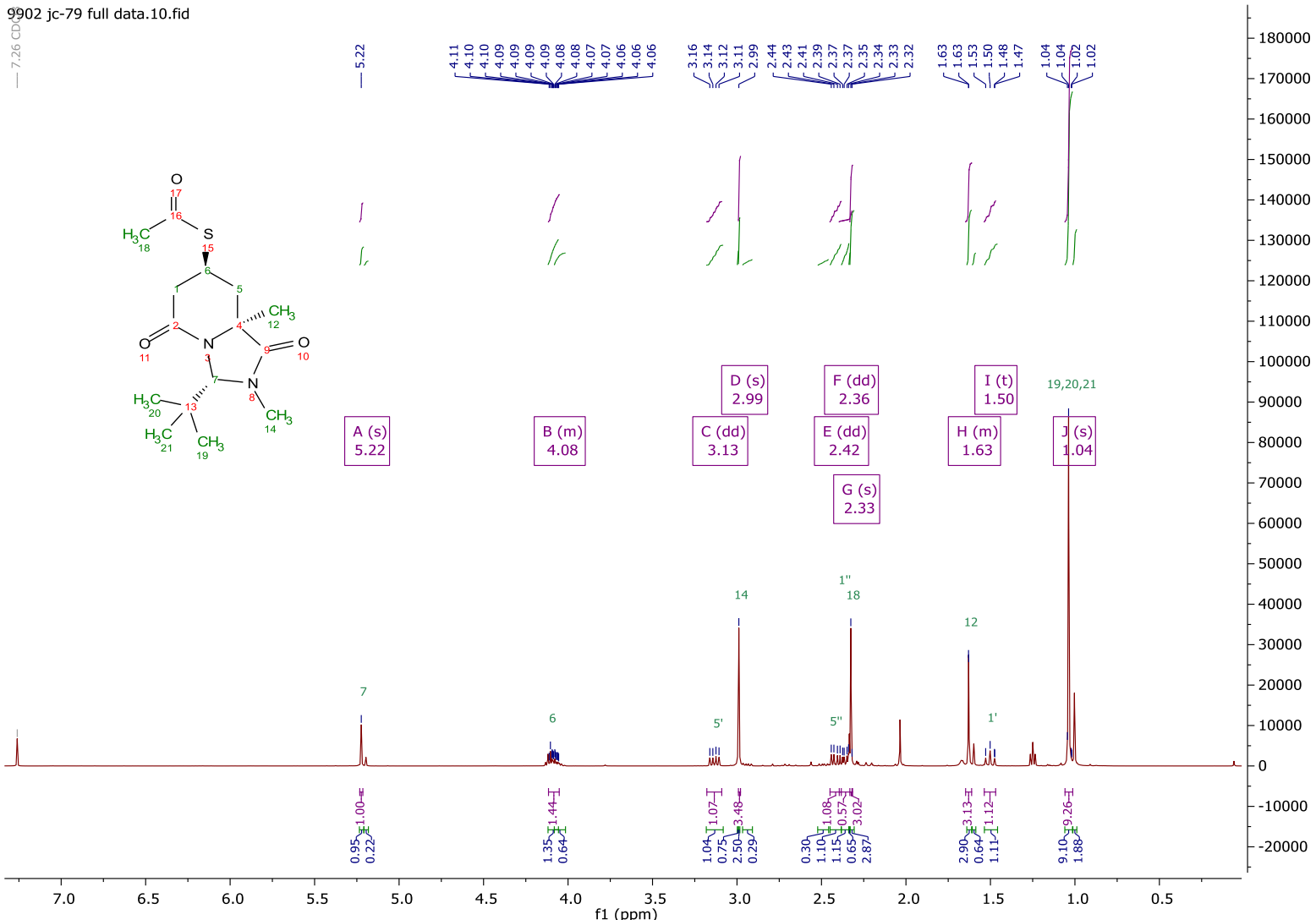

9a

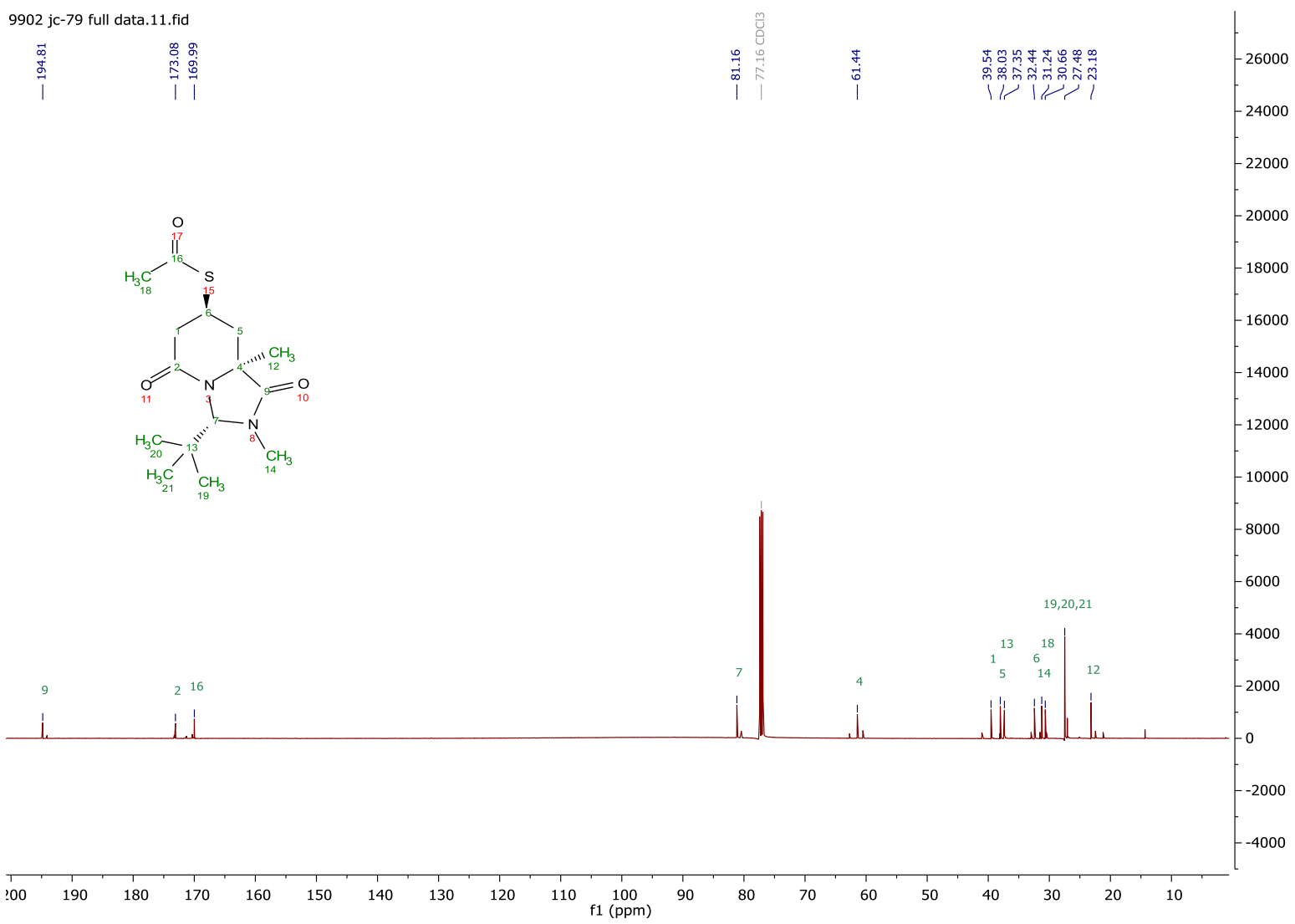


9b

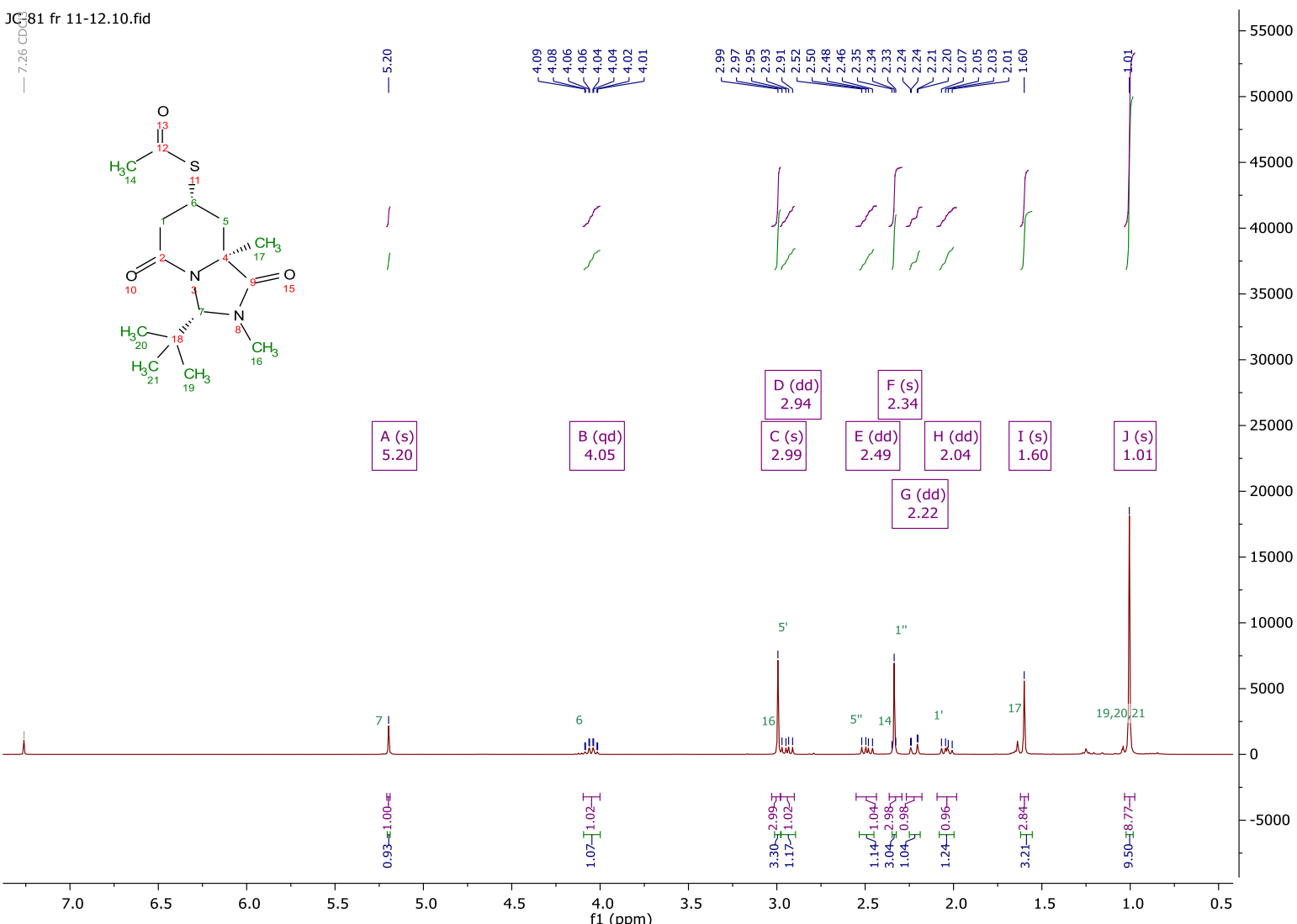

9b

JC-81 full charac.14.fid

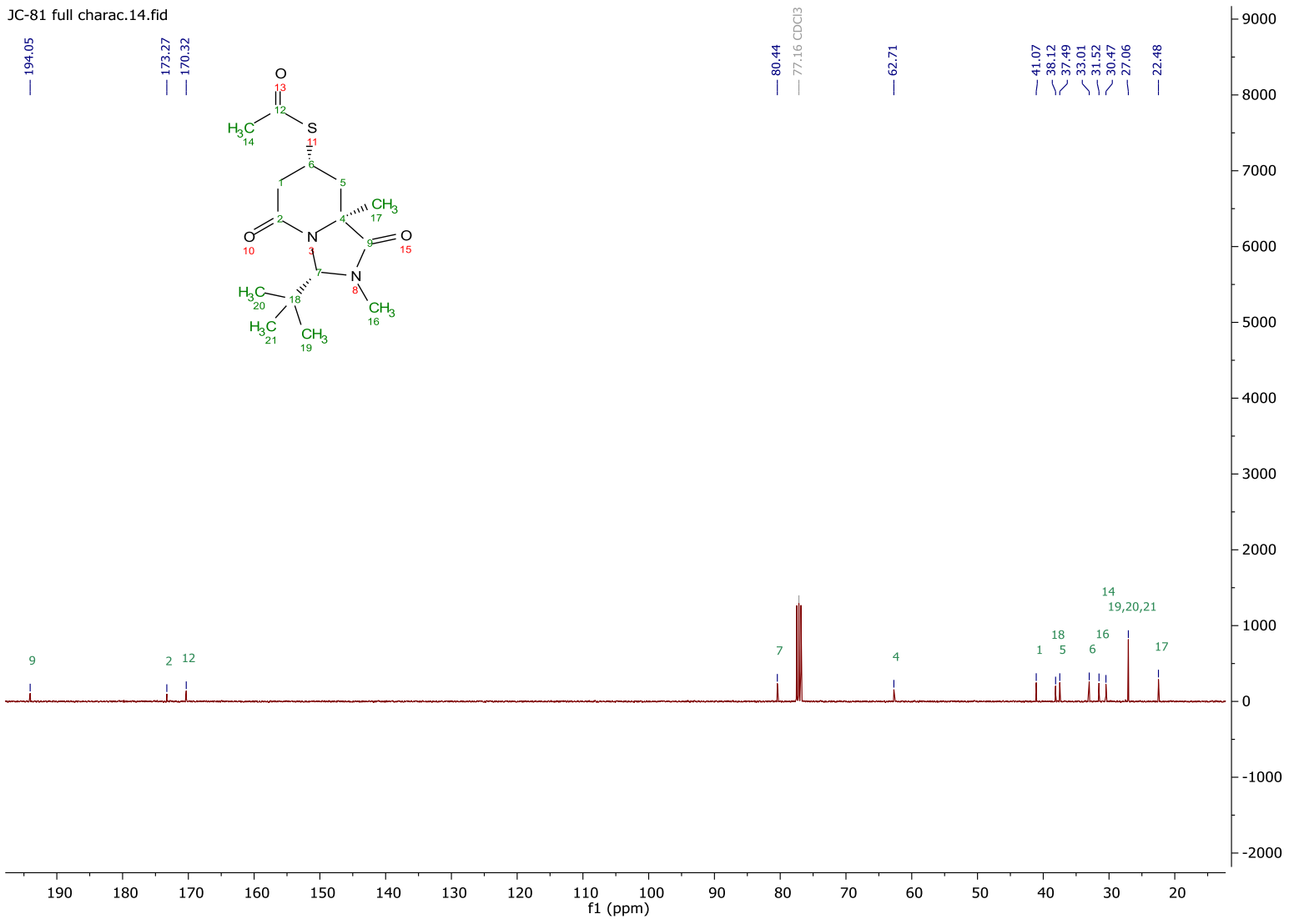


$10 a$

9903 jc-77 full data.10.fid

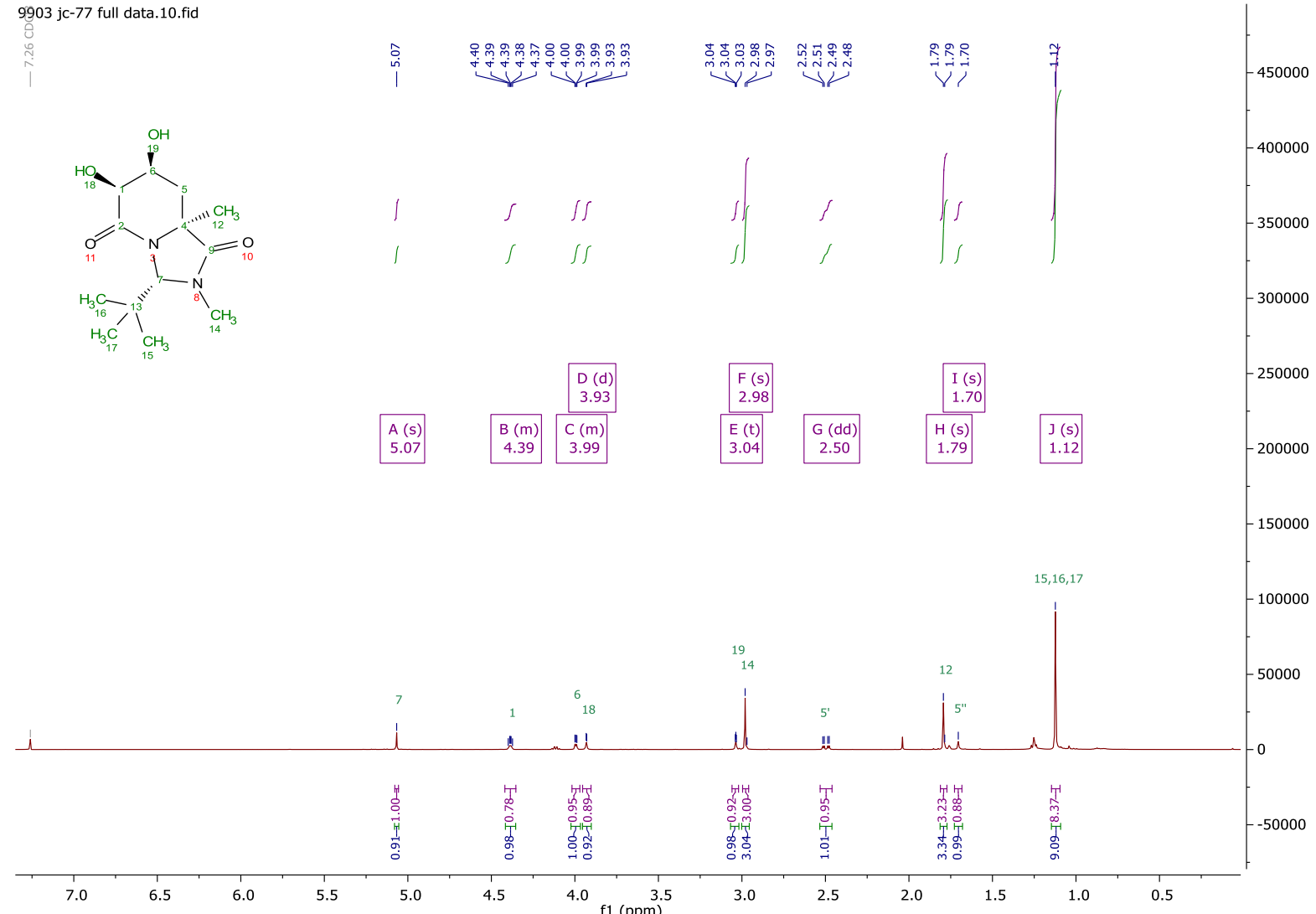

$10 a$

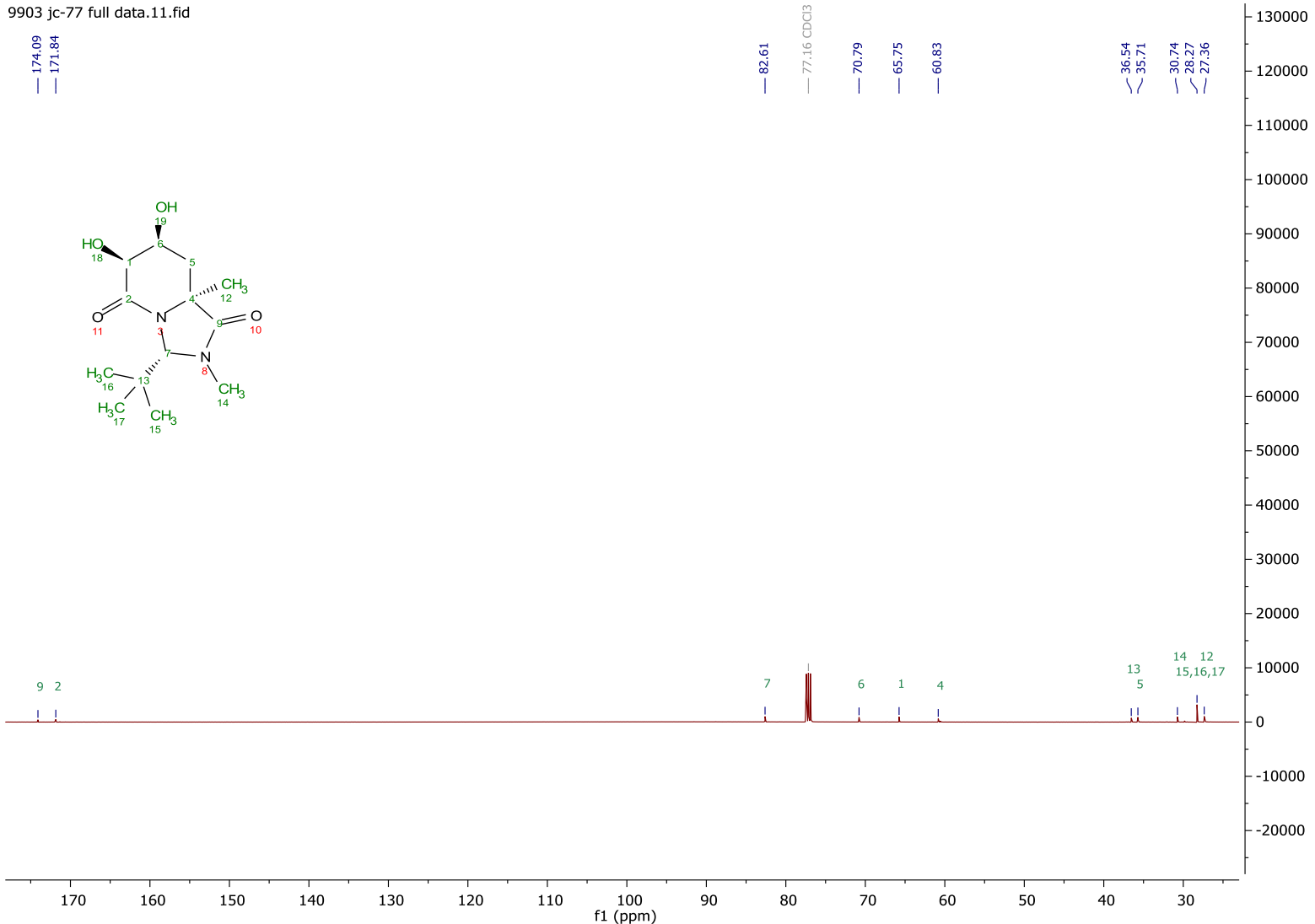


$11 a$

$9901 \mathrm{jc-80}$ pd postwash.10.fid

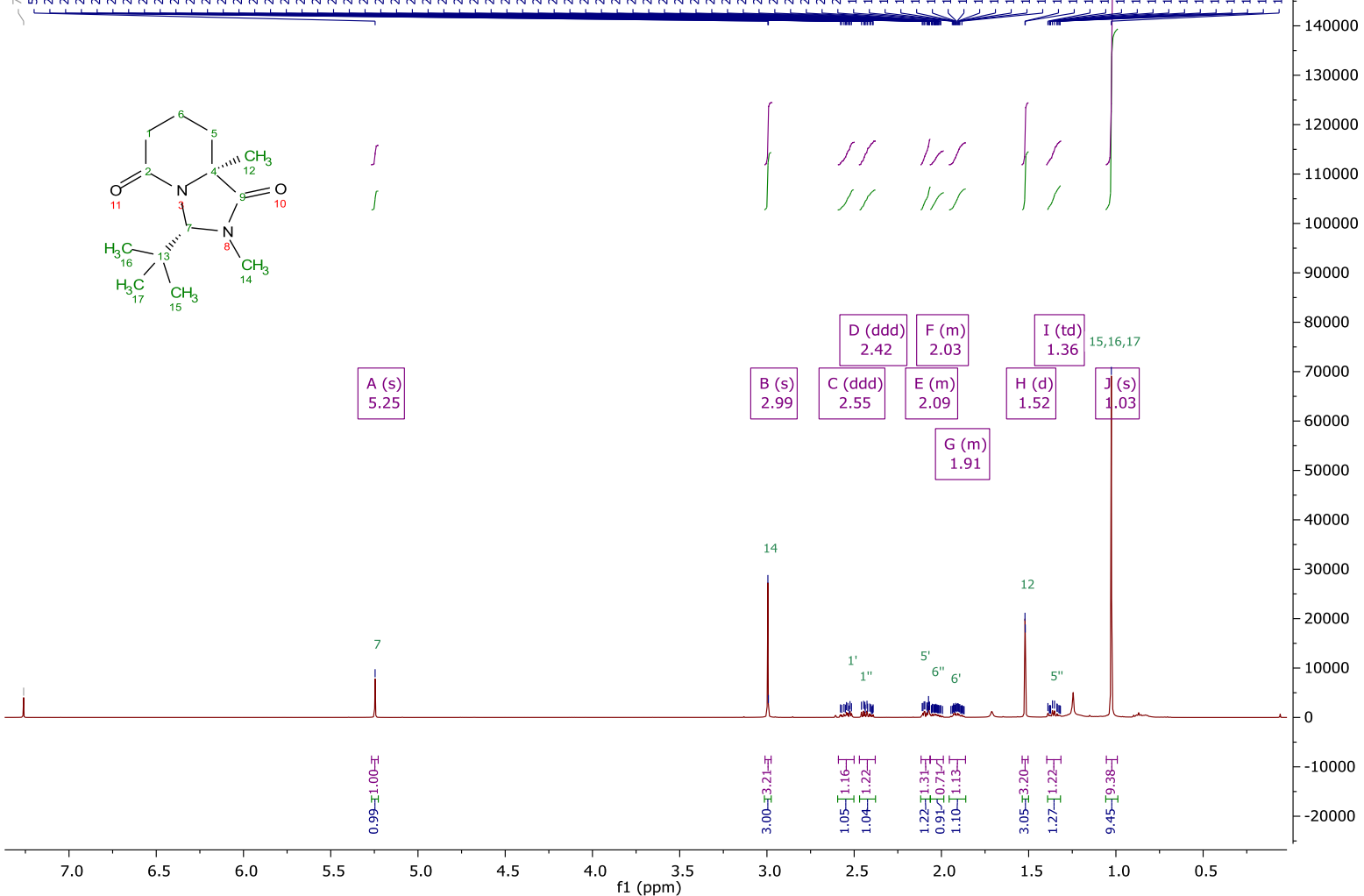

11a

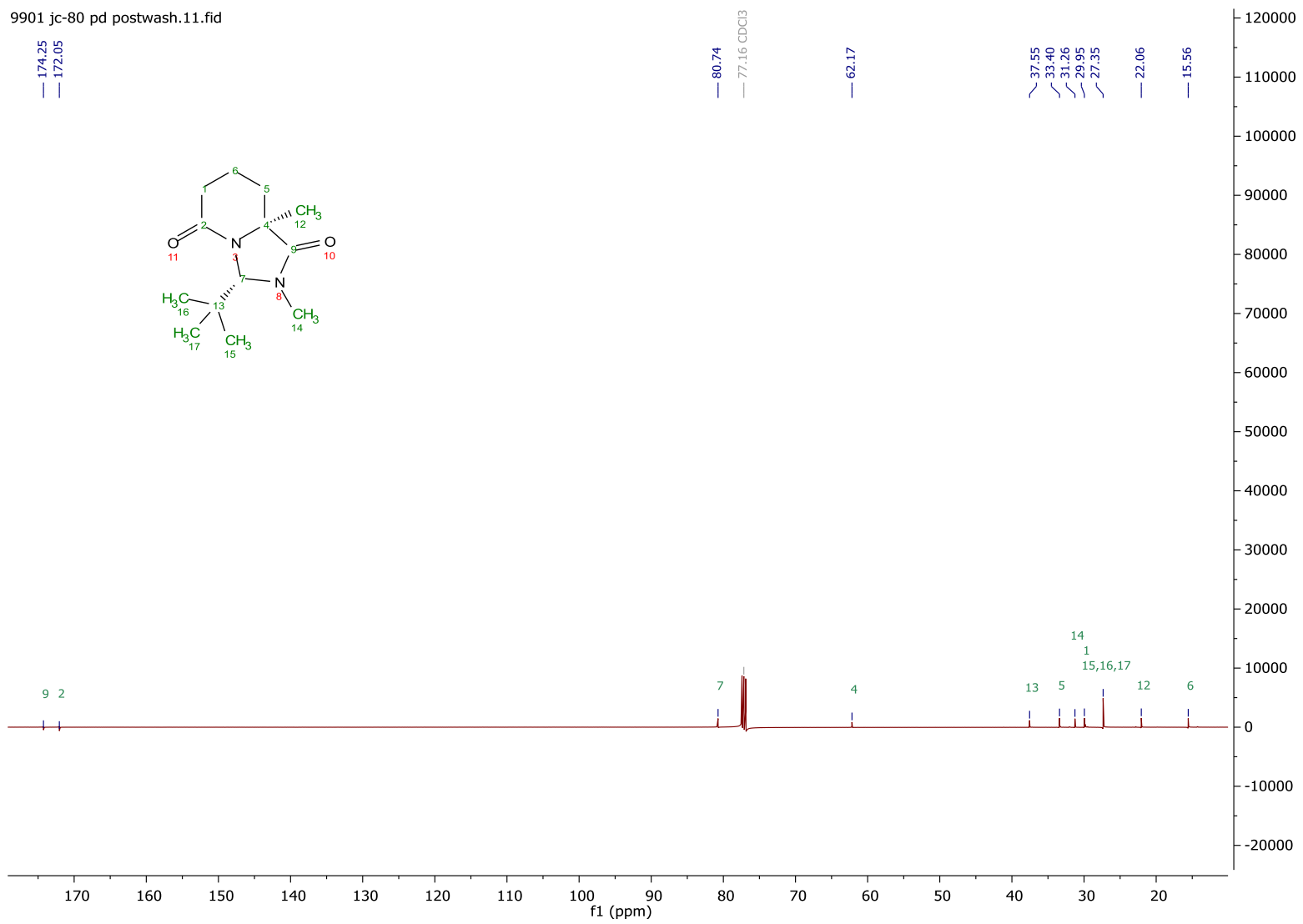


$11 \mathrm{c}$

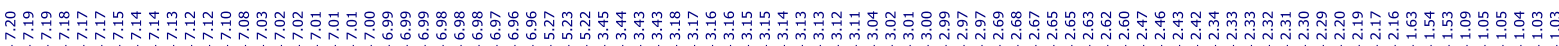

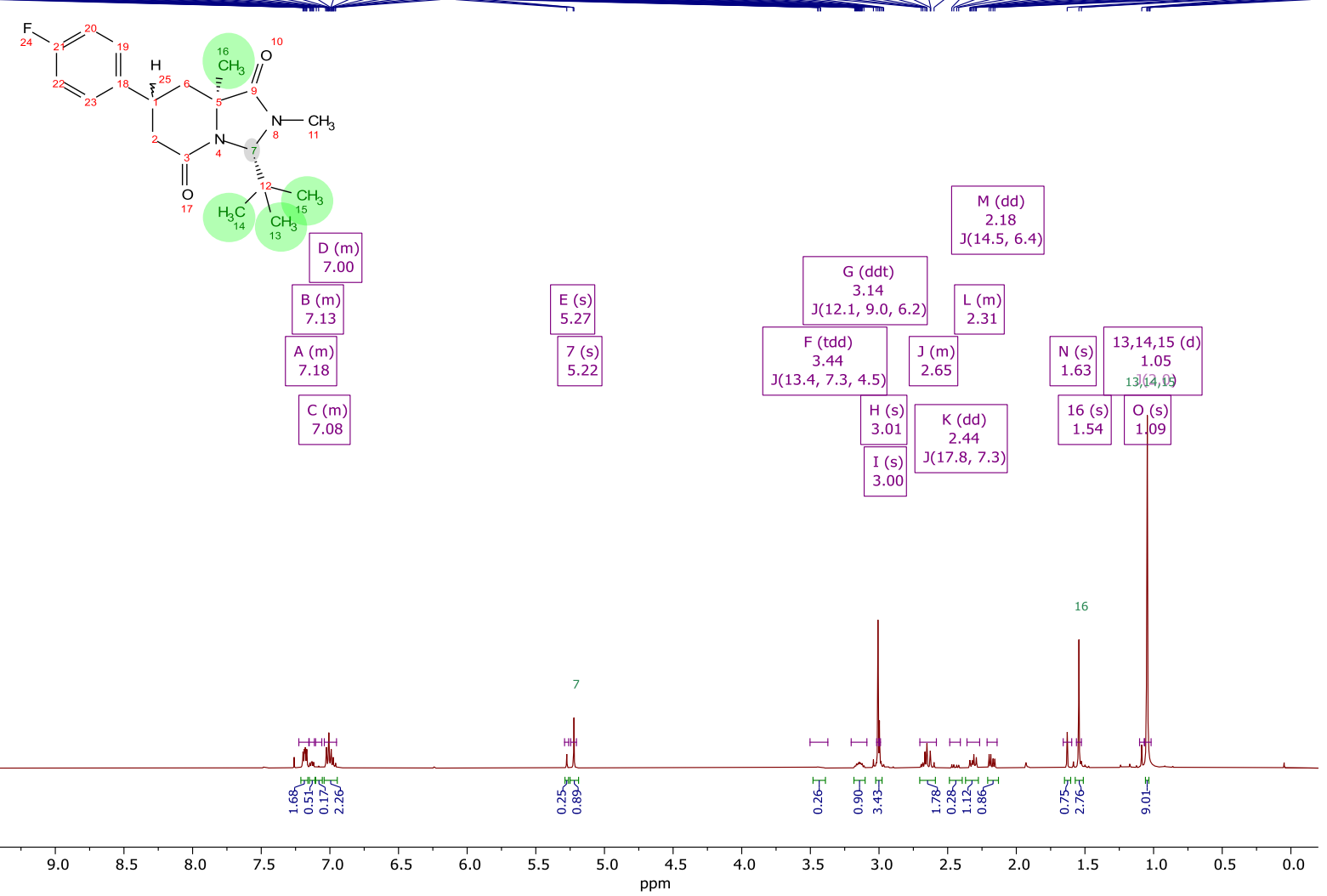

11c

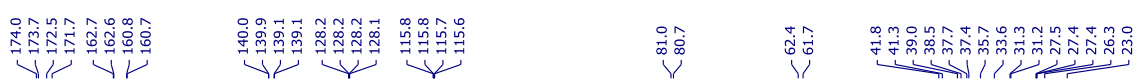
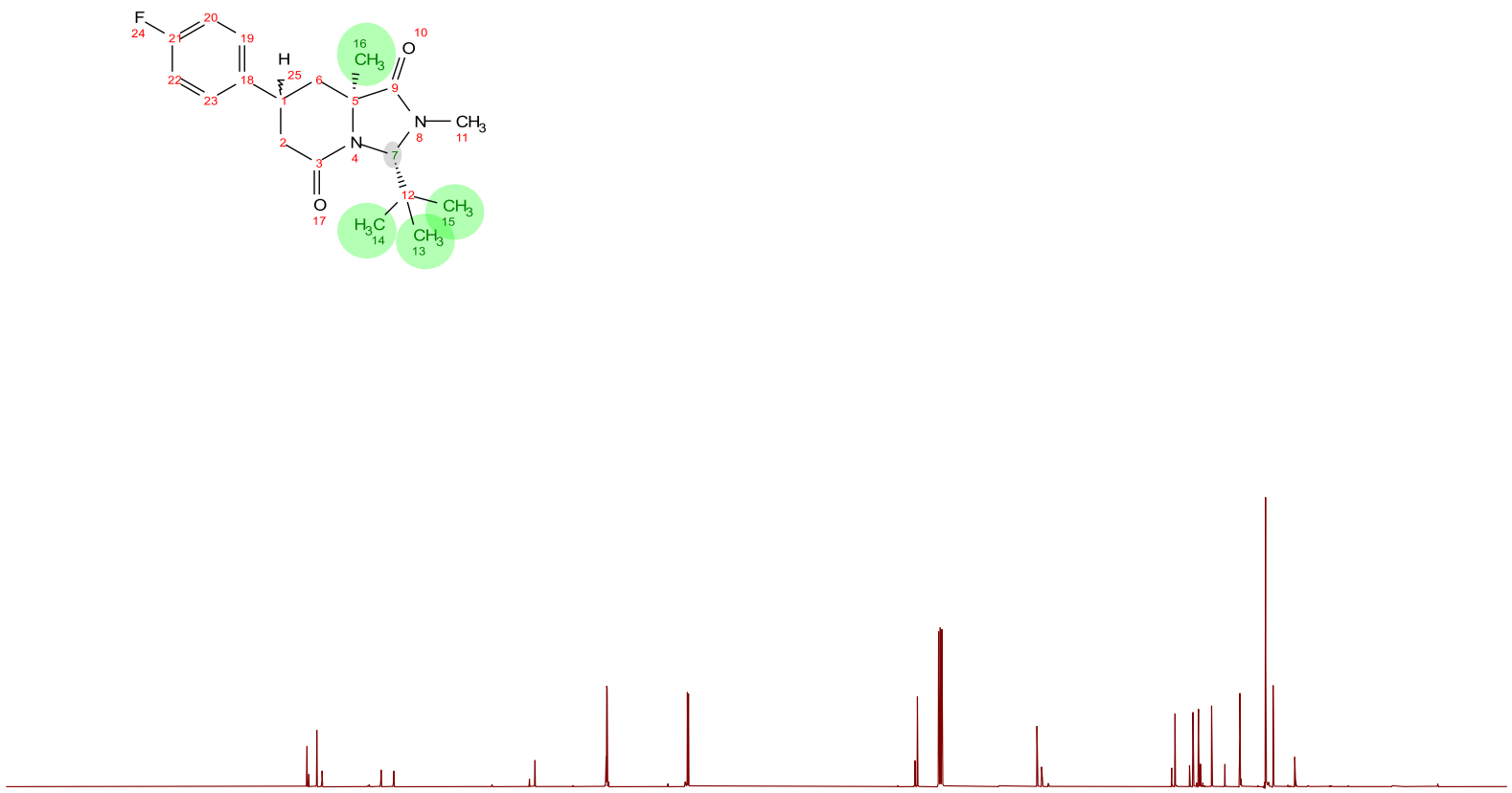

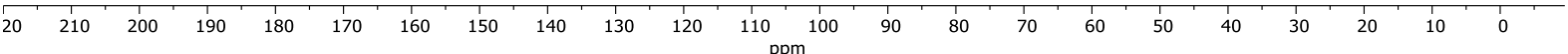


10c

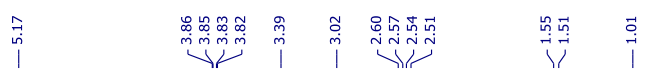

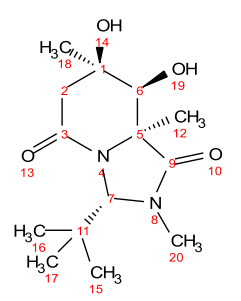

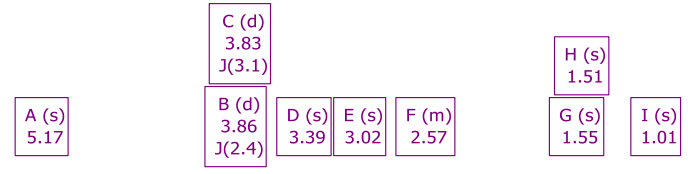

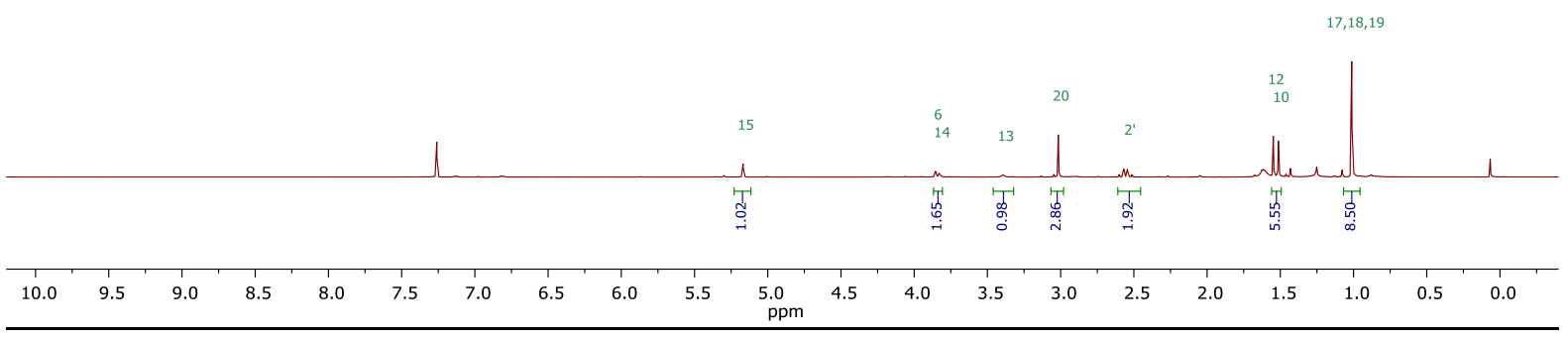

10c

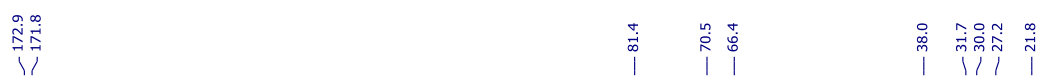

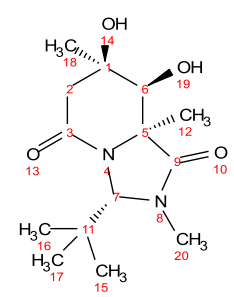

\begin{tabular}{ll|l}
16 & 10 & 12 \\
& &
\end{tabular} 
10b

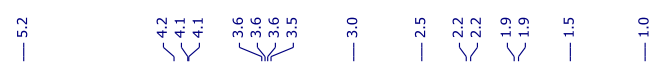

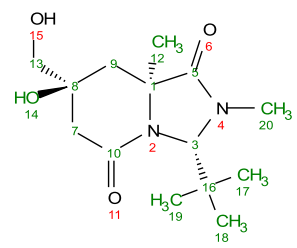

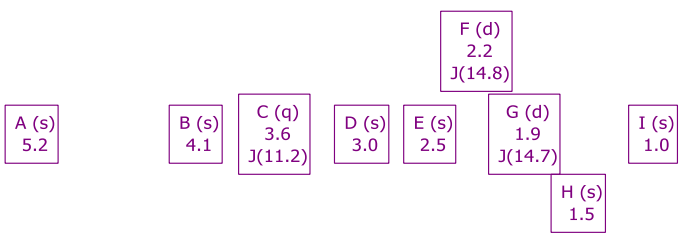

$\underset{18}{17,19}$

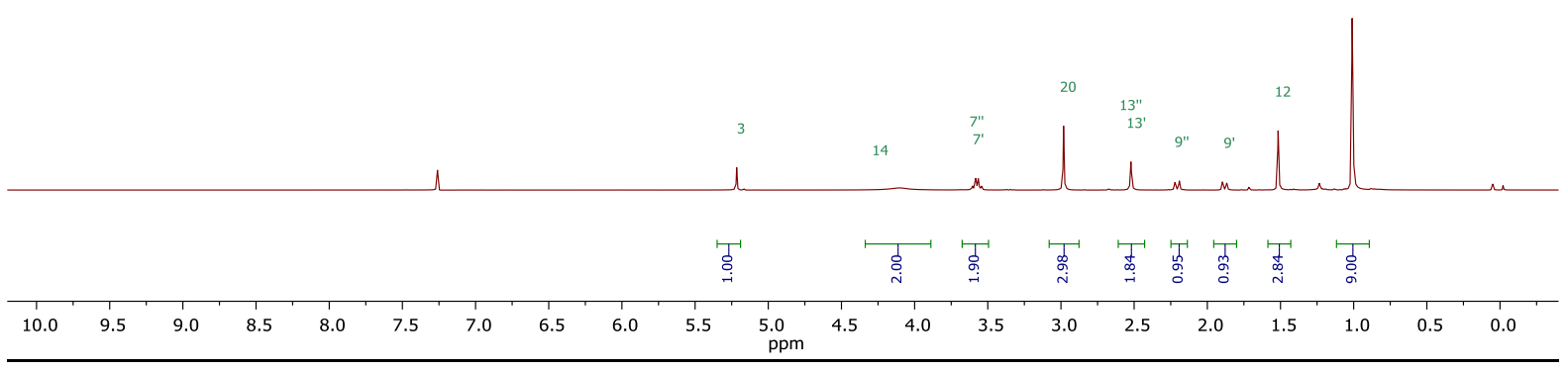

$10 \mathrm{~b}$

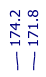

I li li

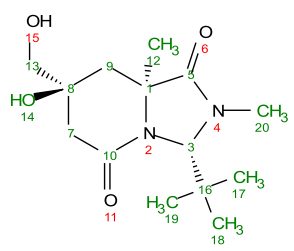

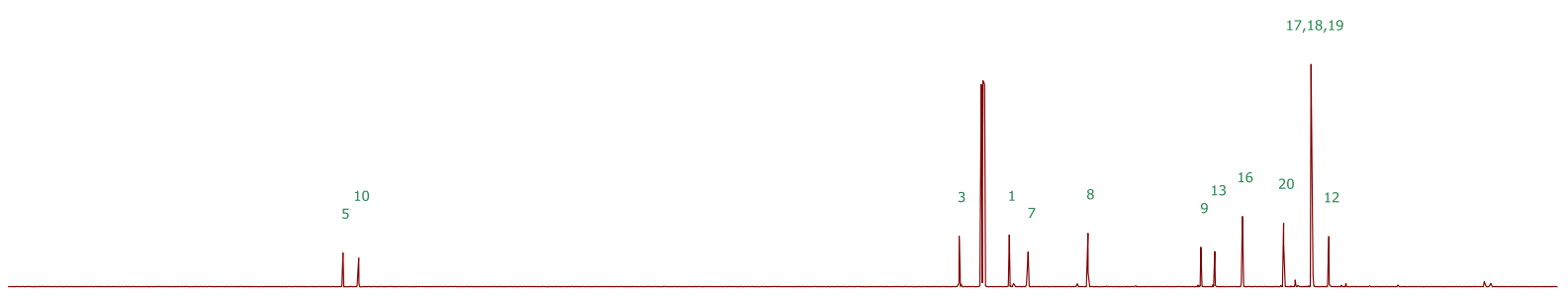

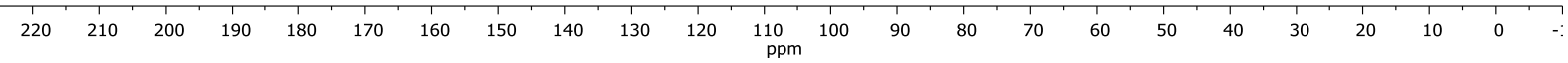


11h
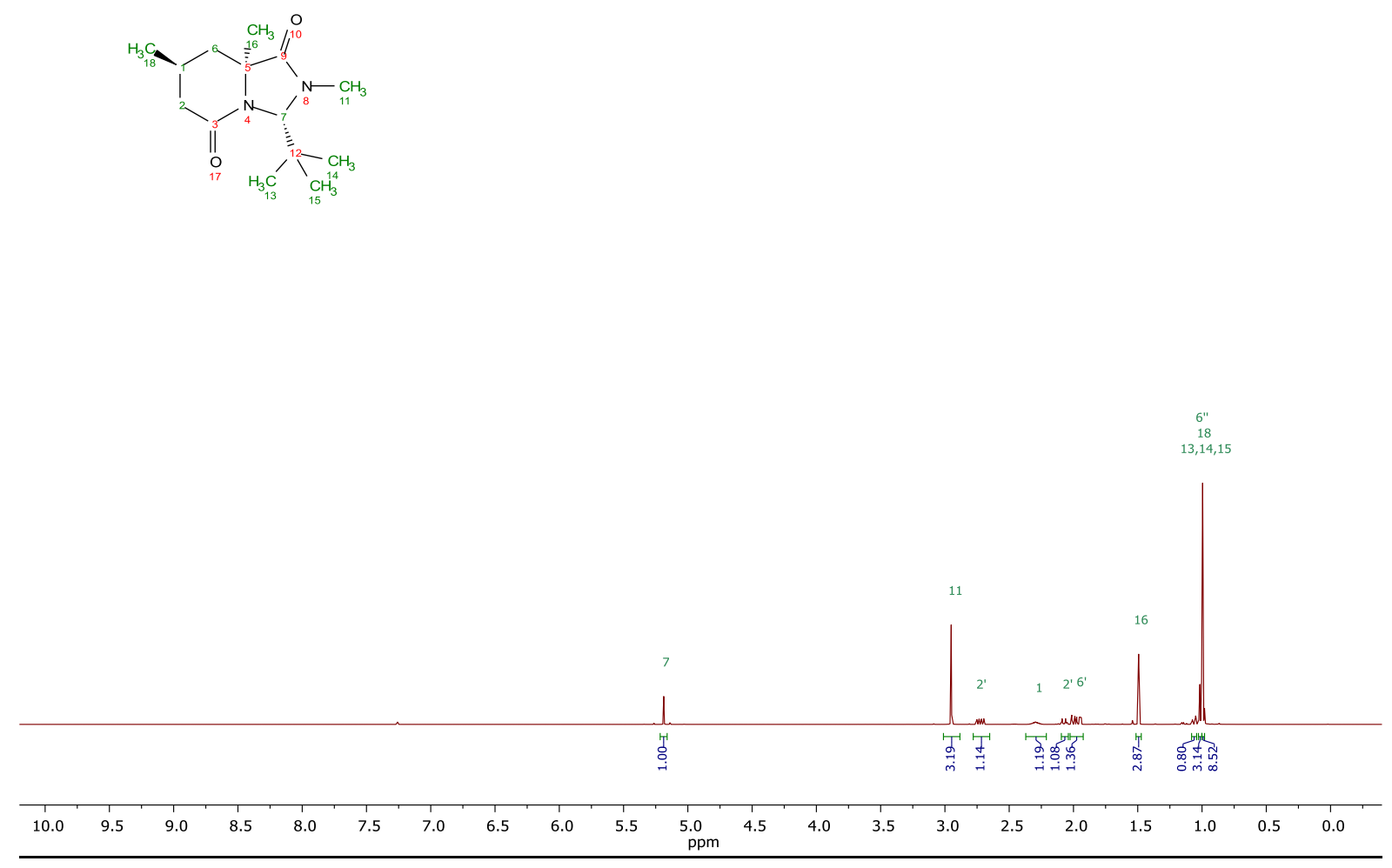

11h
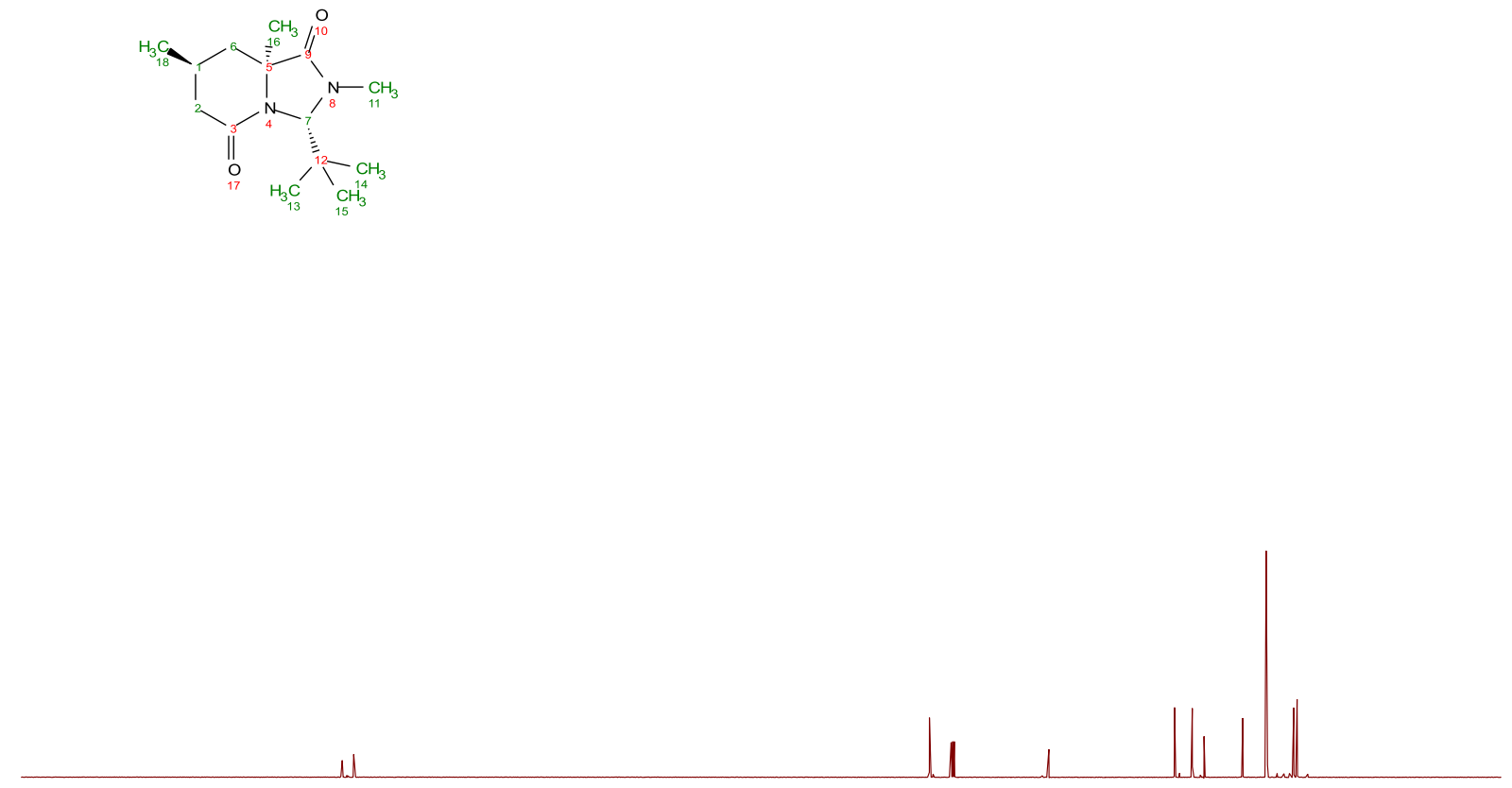

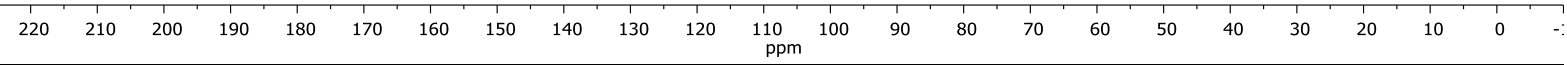


13a

$\underset{\substack{\infty \\ \infty \\ \infty}}{\substack{\infty \\ \mid}}$

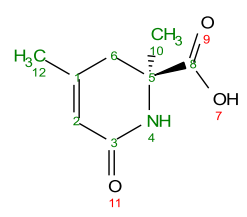

\begin{tabular}{|l|}
\hline A (s) \\
8.89 \\
\hline
\end{tabular}

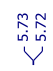

\begin{tabular}{|l|}
\hline$B(s)$ \\
5.72 \\
\hline
\end{tabular}

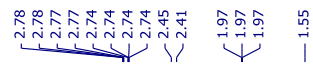

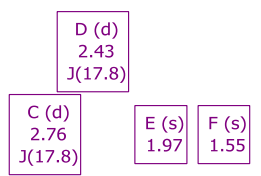

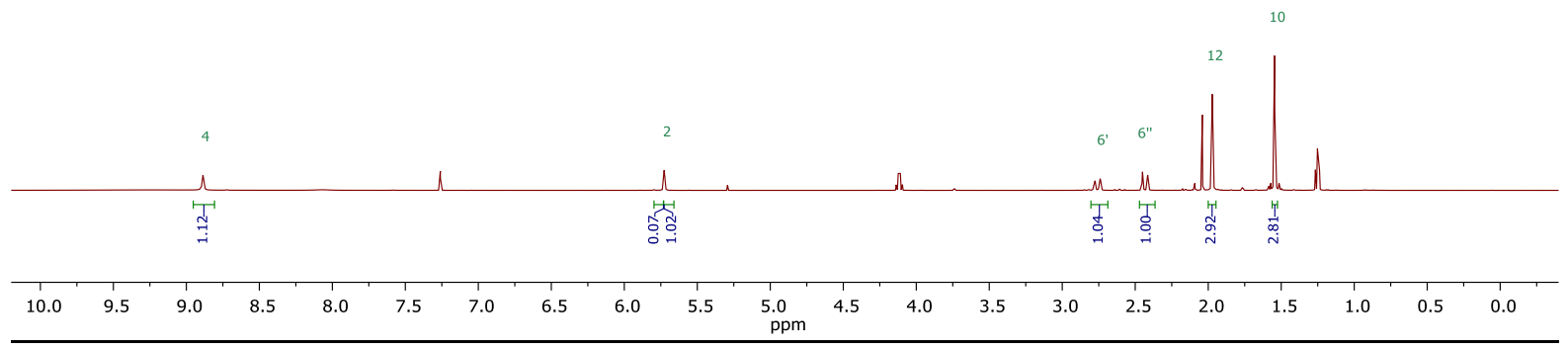

13a
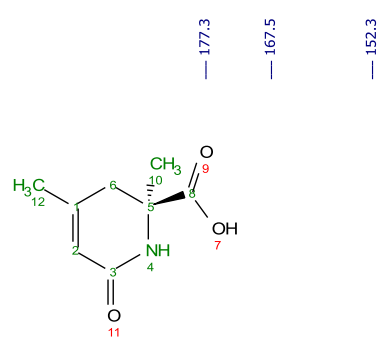

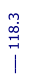

$\hat{i}$

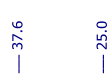

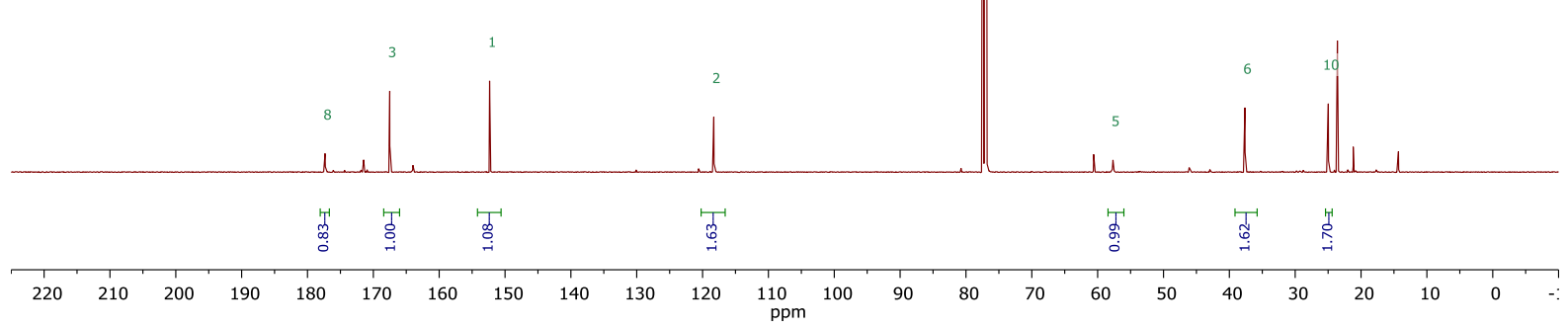

101 
13b

足<smiles>C[C@@H]1CC(=O)N[C@](C)(C(=O)O)C1</smiles>

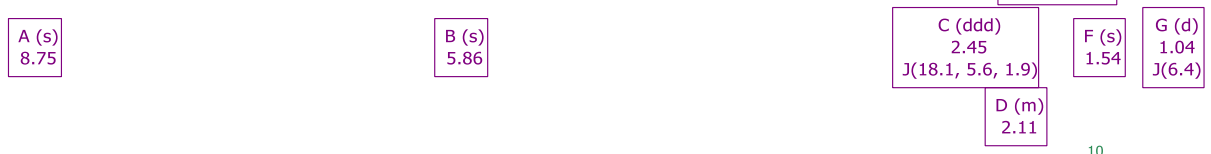
${ }_{60}^{10}$

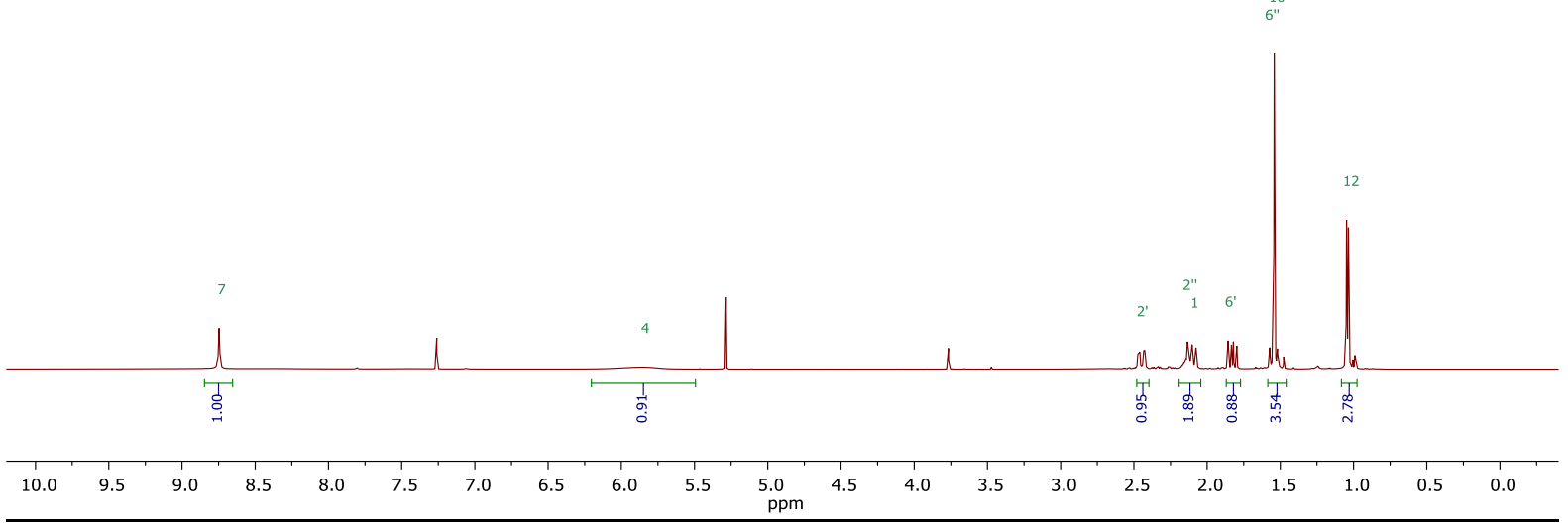

13b

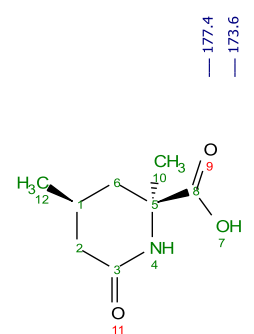

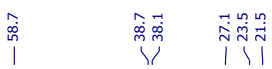

$\stackrel{1}{\stackrel{1}{\sim}}$ 
13c

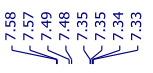

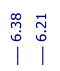

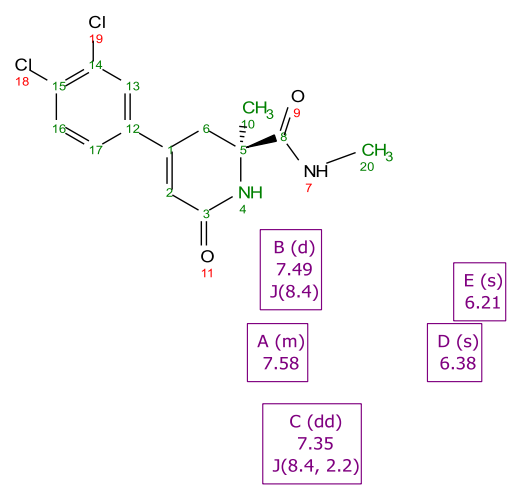

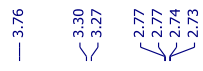

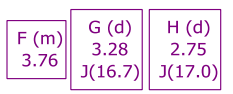

I (s)

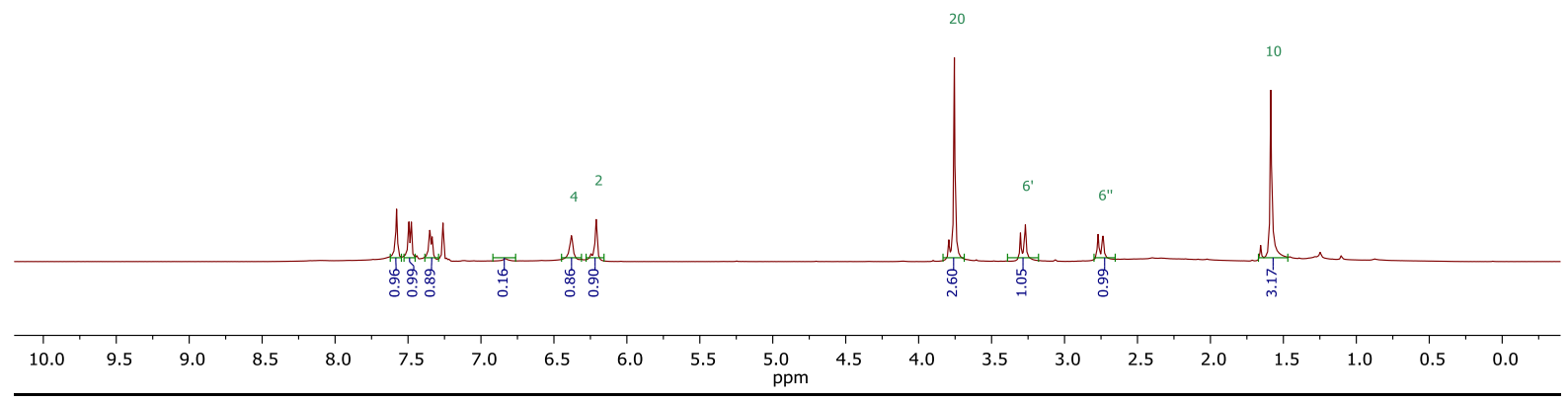

13c
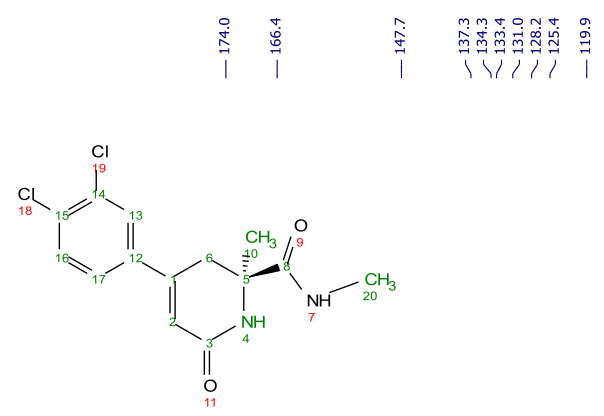

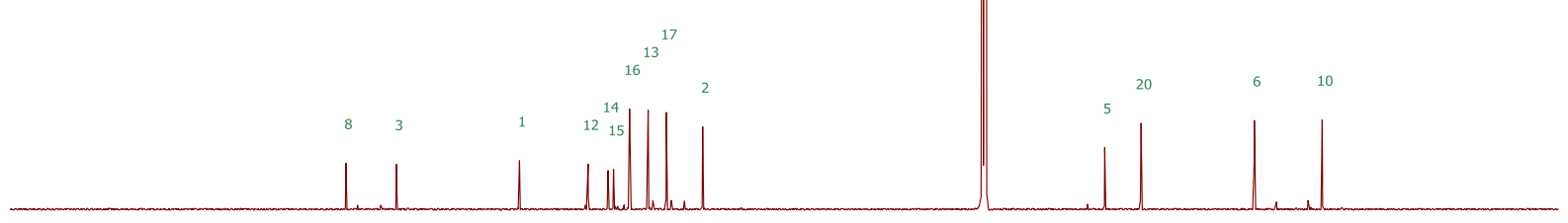

\begin{tabular}{|llllllllllllllllllllllllllllllll}
\hline 220 & 210 & 200 & 190 & 180 & 170 & 160 & 150 & 140 & 130 & 120 & 110 & 100 & 90 & 80 & 70 & 60 & 50 & 40 & 30 & 20 & 10 & 0 & $-:$ \\
\hline
\end{tabular}

103 
13d
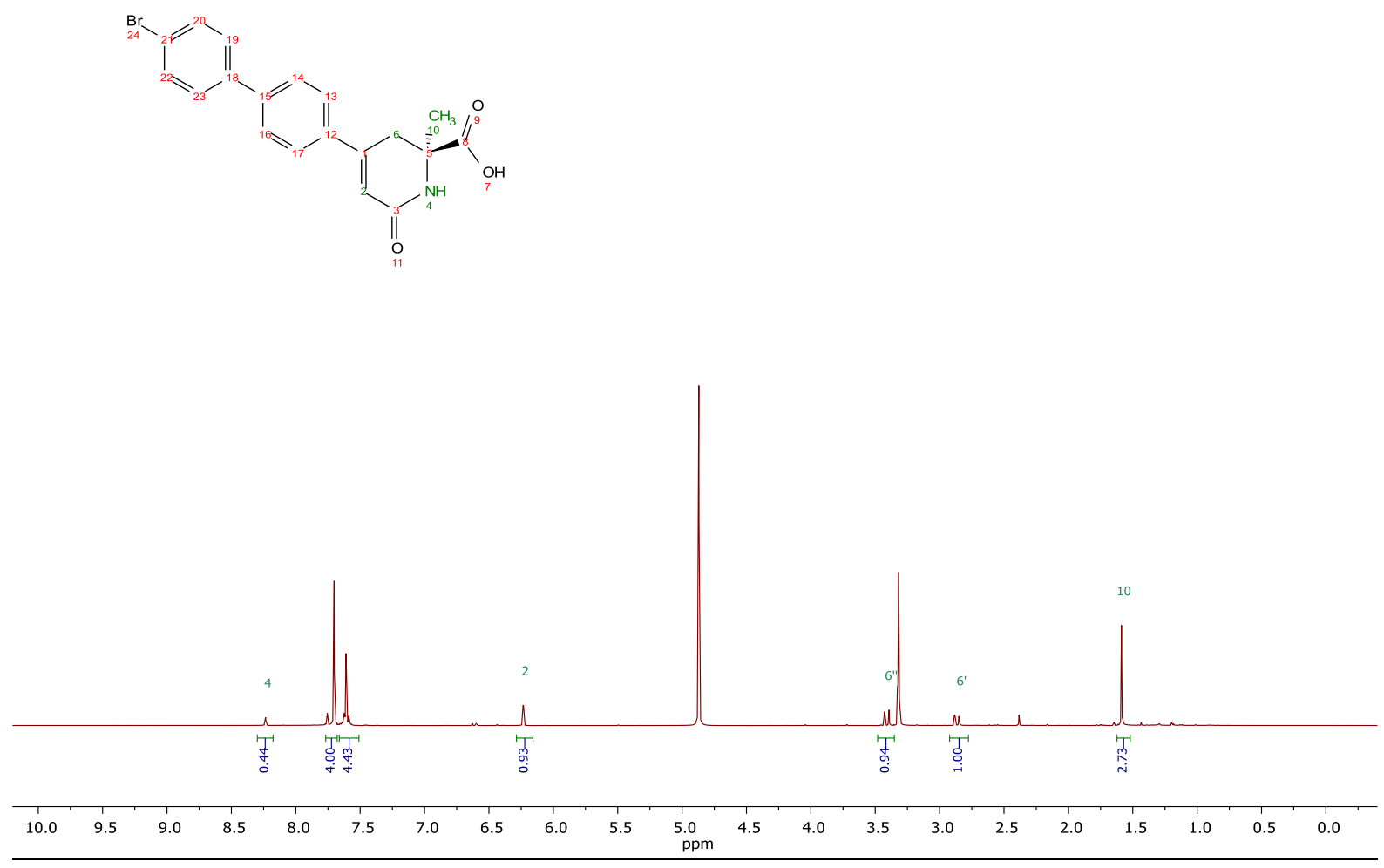

13d
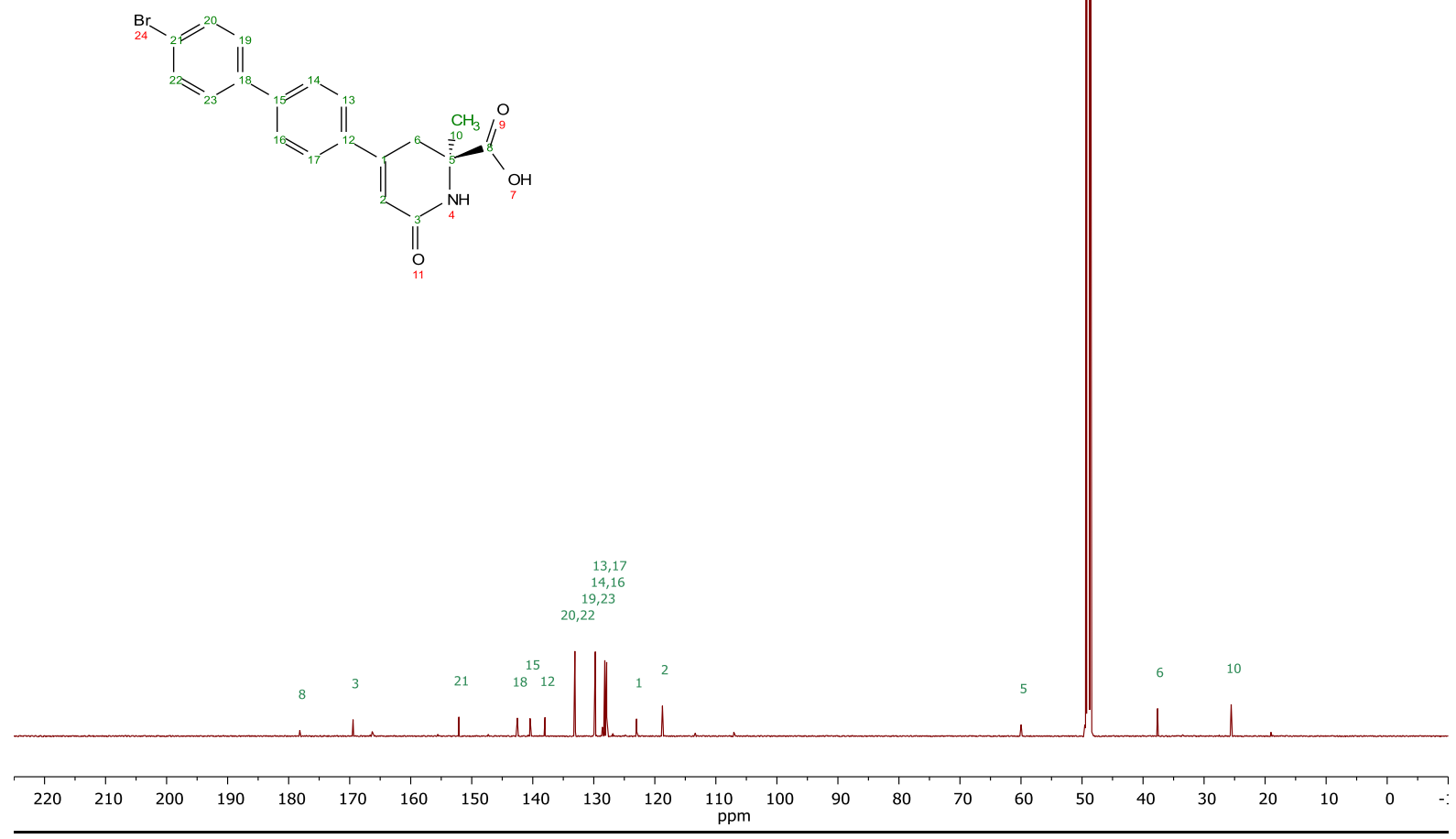

104 
$13 \mathrm{e}$

Y

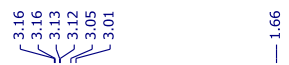

每)

\begin{tabular}{|l|l|l|l|}
\hline A (s) & B (m) & C (m) & D (s) \\
9.09 & 7.56 & 7.13 & 6.23 \\
\hline
\end{tabular}

\begin{tabular}{|c|}
\hline$G(d)$ \\
3.03 \\
$J(17.4)$ \\
\hline$E(d d)$ \\
3.14 \\
$J(17.4,2.4)$ \\
\hline
\end{tabular}

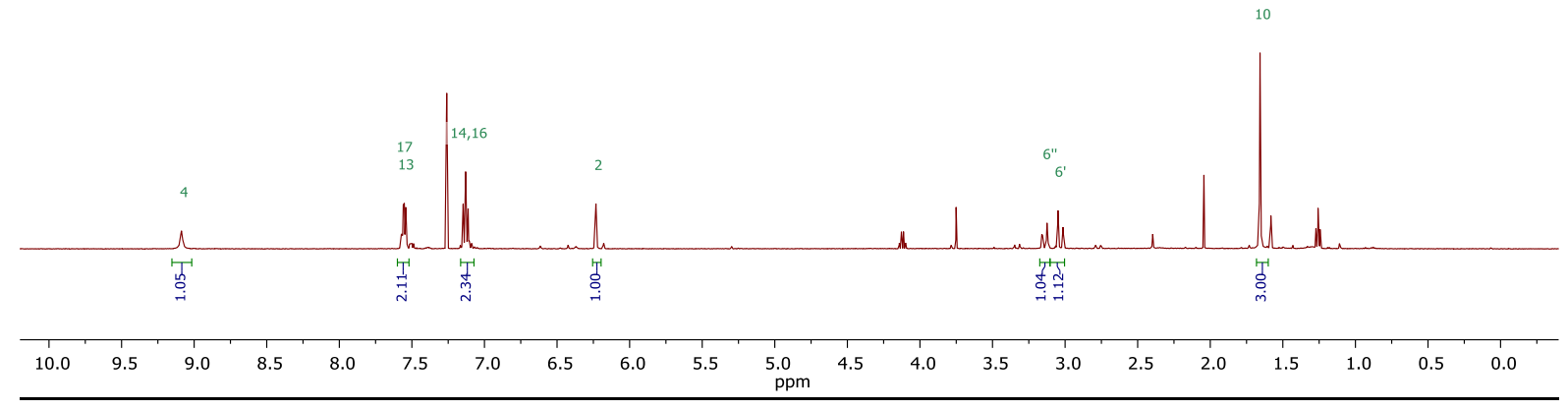

$13 e$<smiles>O=C1C=C(n2ccc(F)n2)CC(C(=O)O)(C(=O)O)N1</smiles>

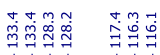

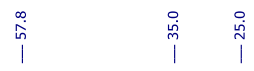

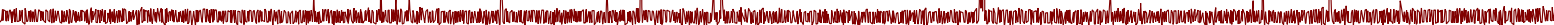

$\begin{array}{llllllllllllllllllllllllll}220 & 210 & 200 & 190 & 180 & 170 & 160 & 150 & 140 & 130 & 120 & 110 & 100 & 90 & 80 & 70 & 60 & 50 & 40 & 30 & 20 & 10 & 0 & -\end{array}$


NOE Analysis

\section{Compound 2a}

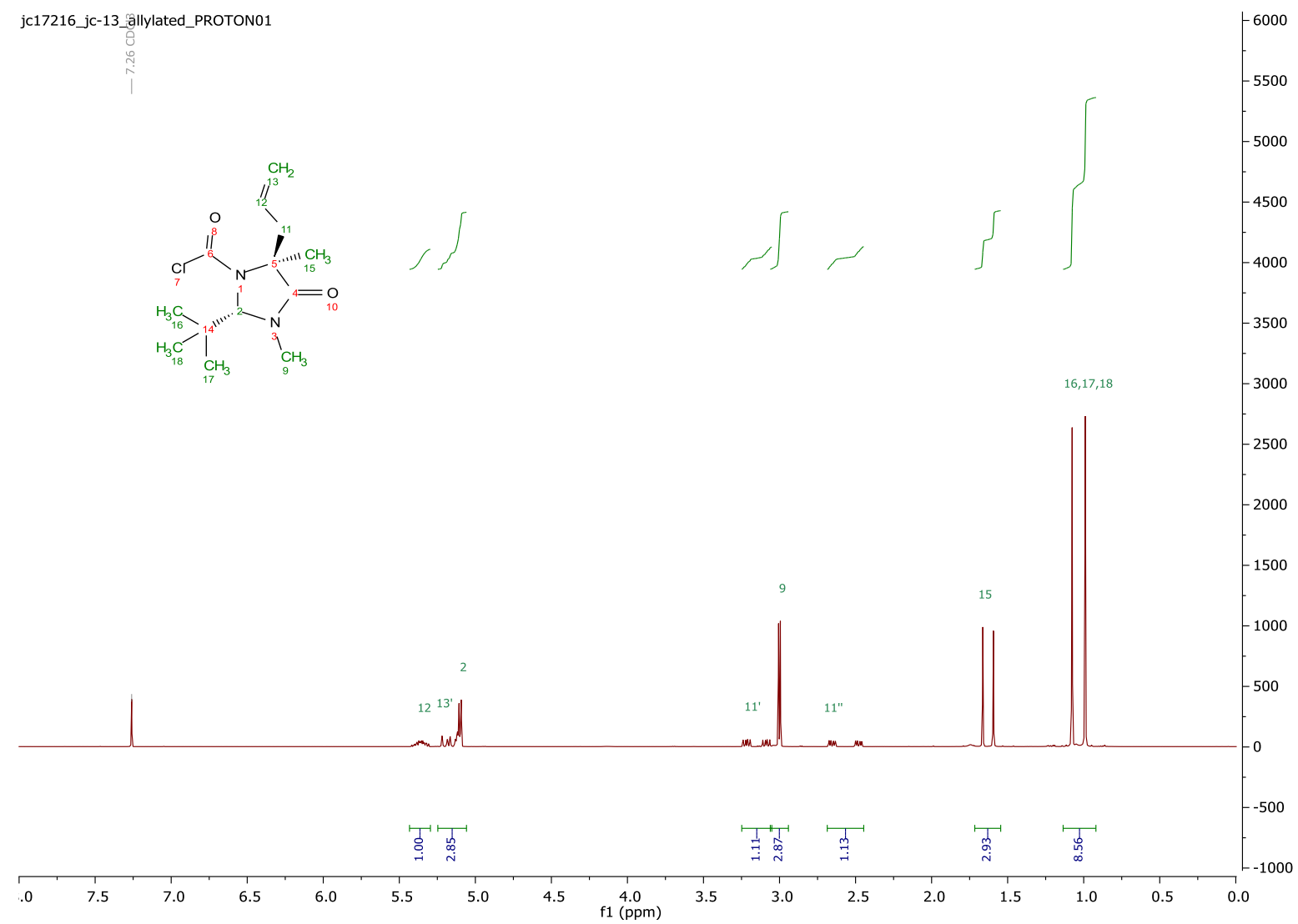

jc17216_jc-13_allylated_NOESY1D01

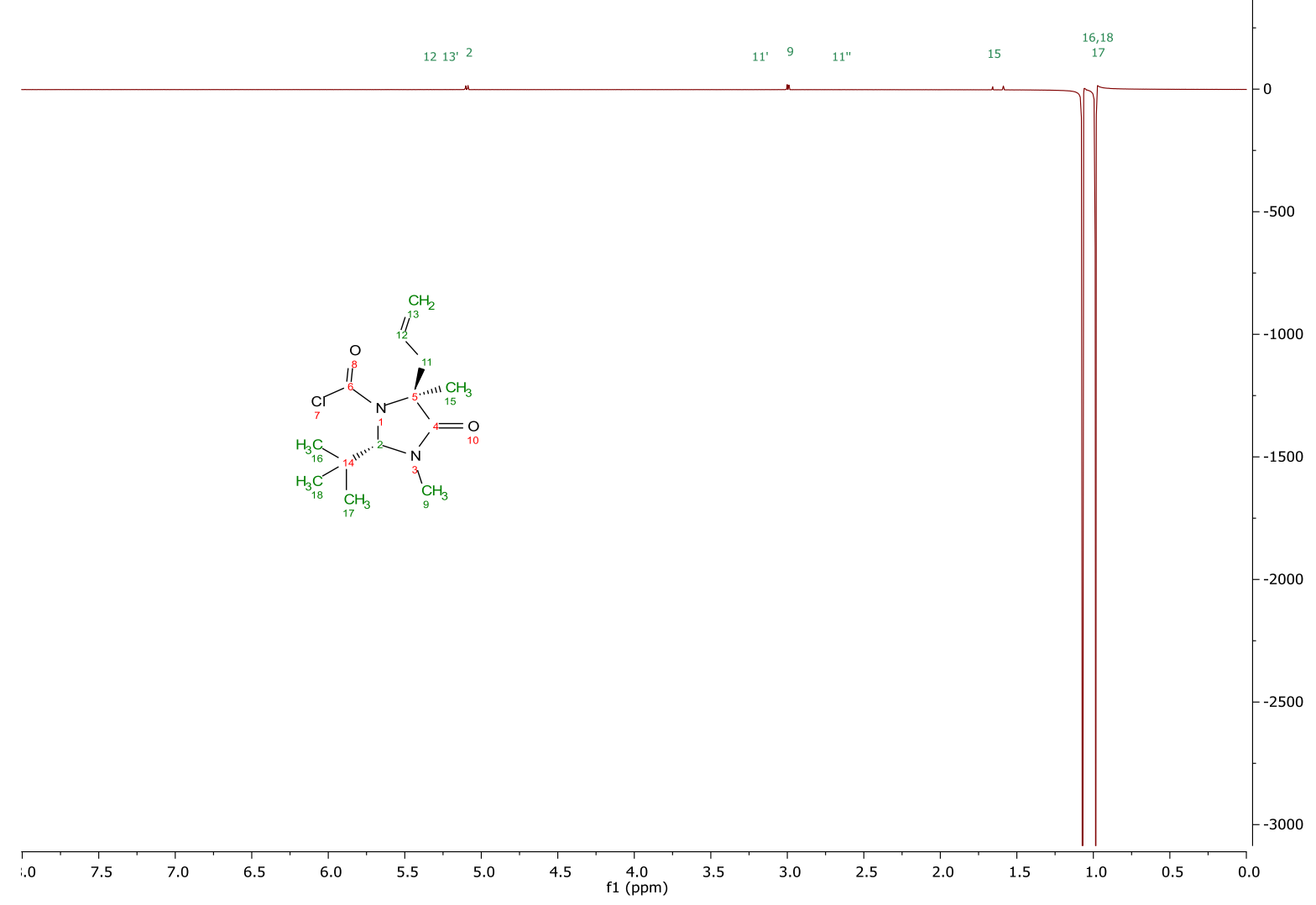




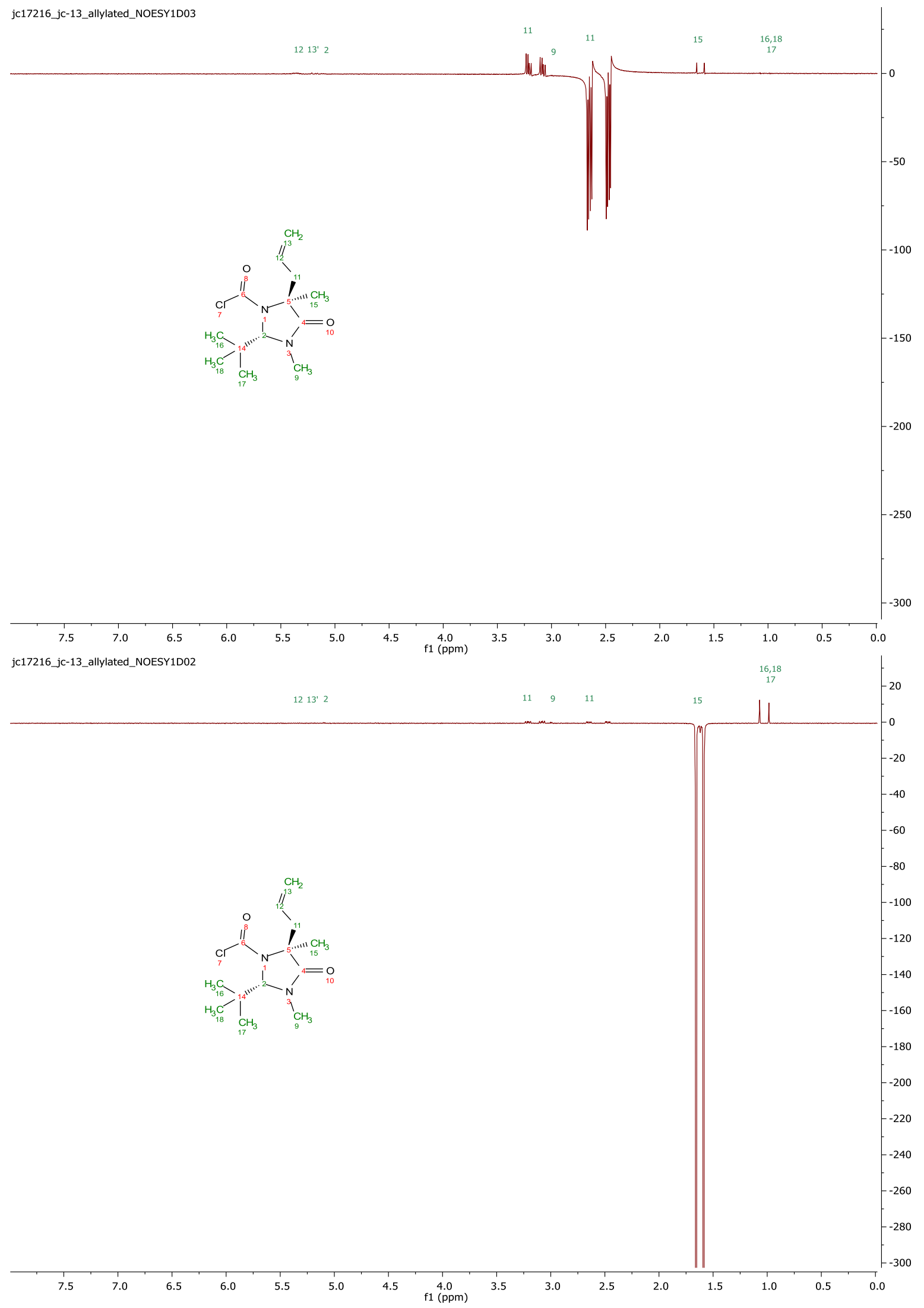

107 


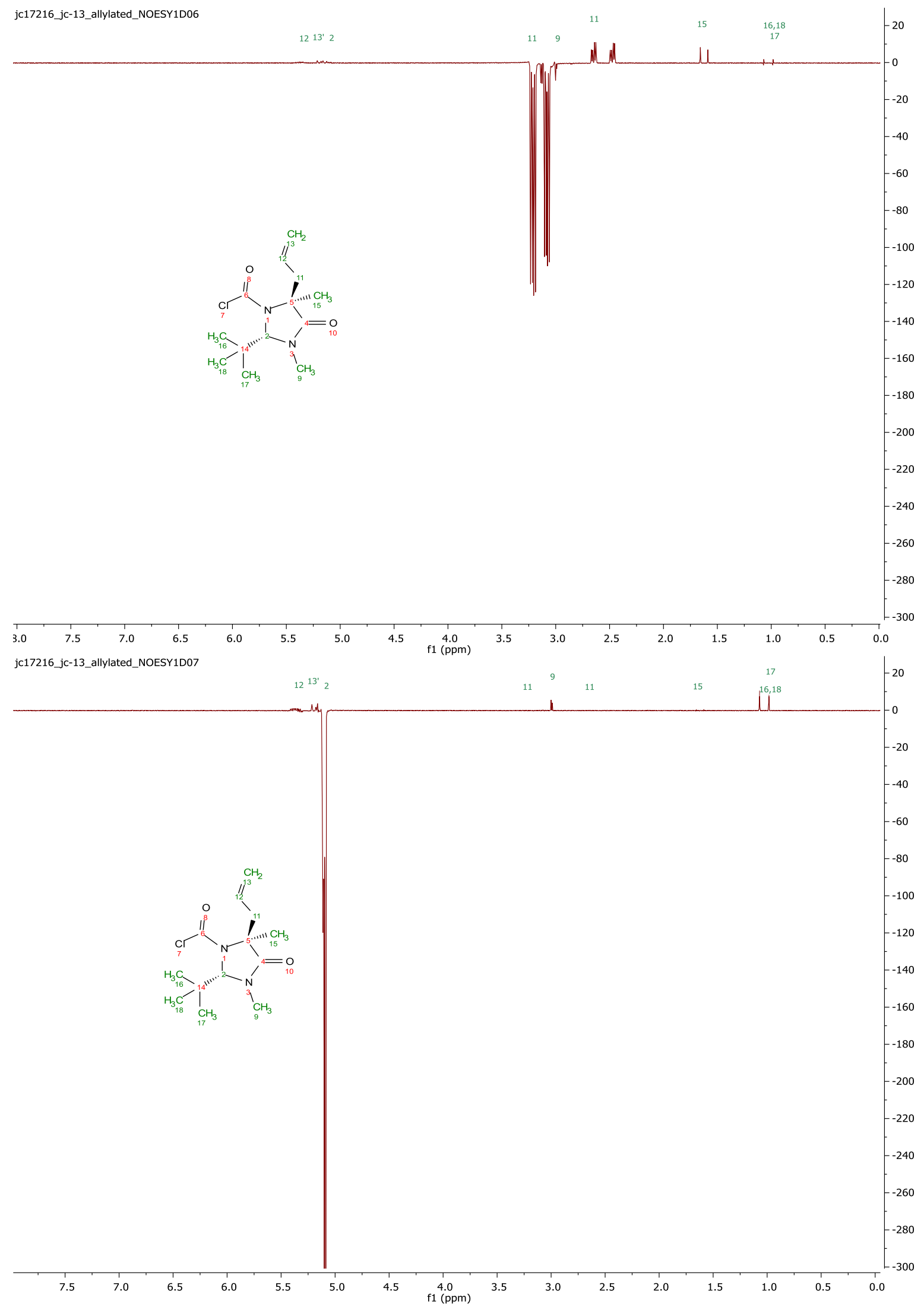




\section{Compound 3b}
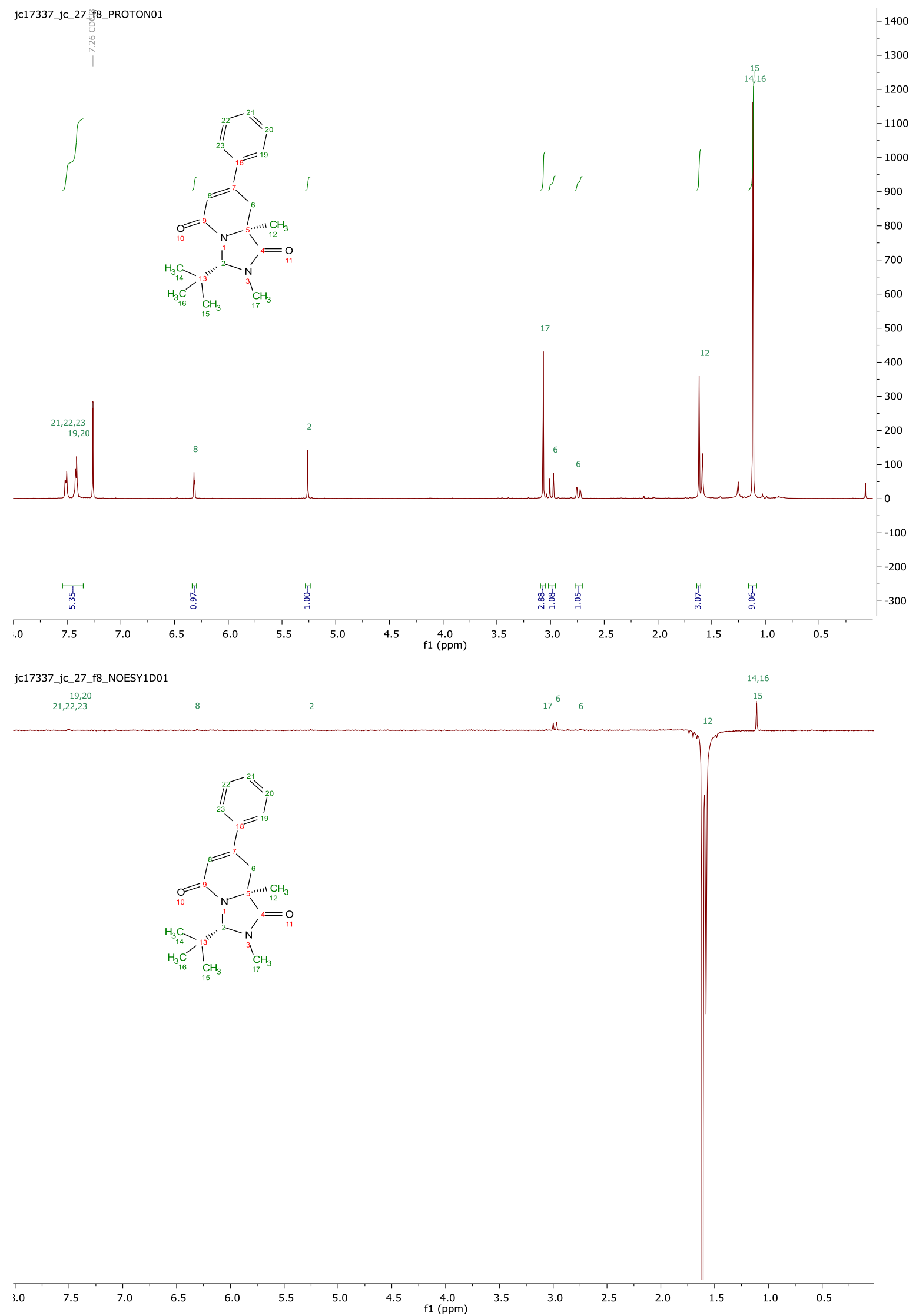


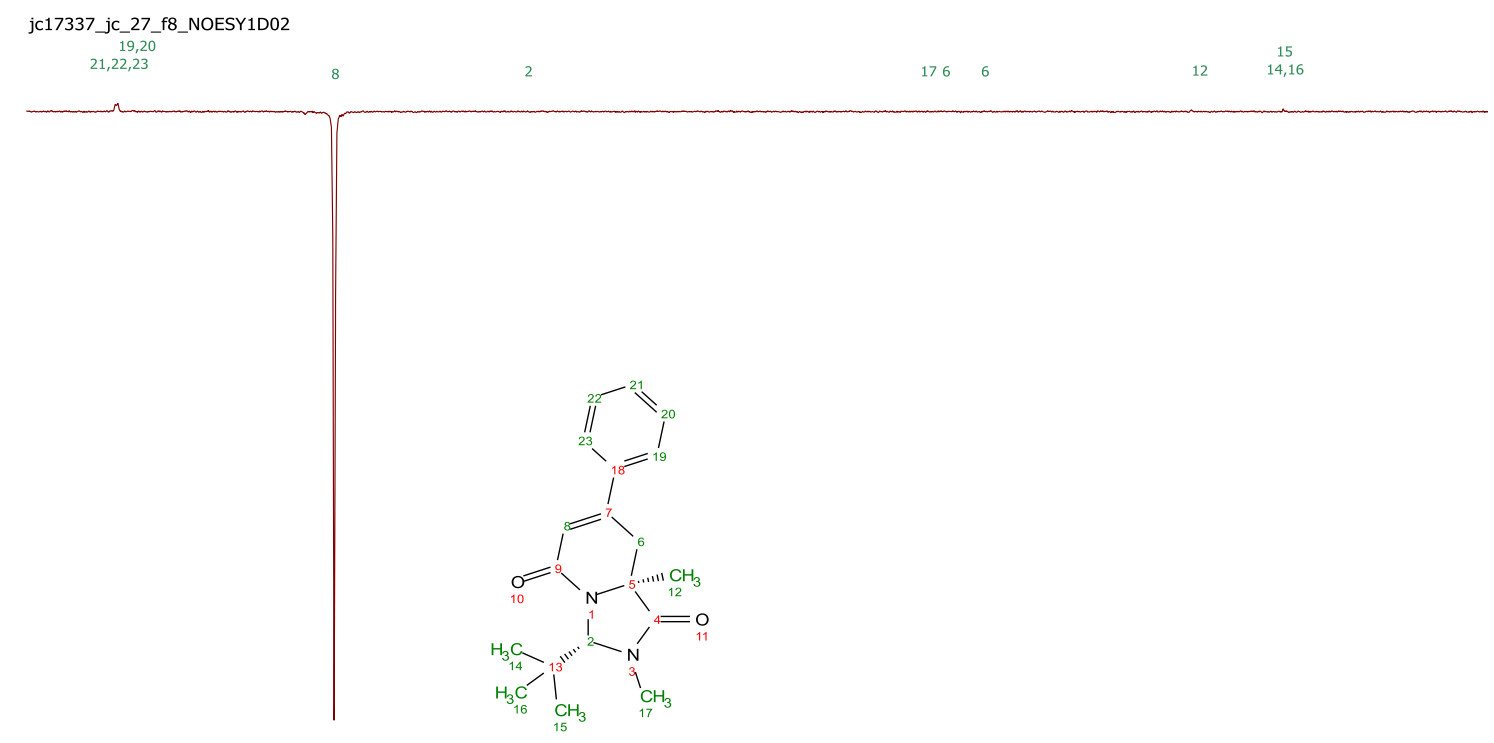

\begin{tabular}{|c|c|c|c|c|c|c|c|c|c|c|c|c|c|c|c|}
\hline .0 & 7.5 & 7.0 & 6.5 & 6.0 & 5.5 & 5.0 & 4.5 & $\begin{array}{c}4.0 \\
\mathrm{f} 1(\mathrm{ppm})\end{array}$ & 3.5 & 3.0 & 2.5 & 2.0 & 1.5 & 1.0 & 0.5 \\
\hline
\end{tabular}

jc17337_jc_27_f8_NOESY1D03

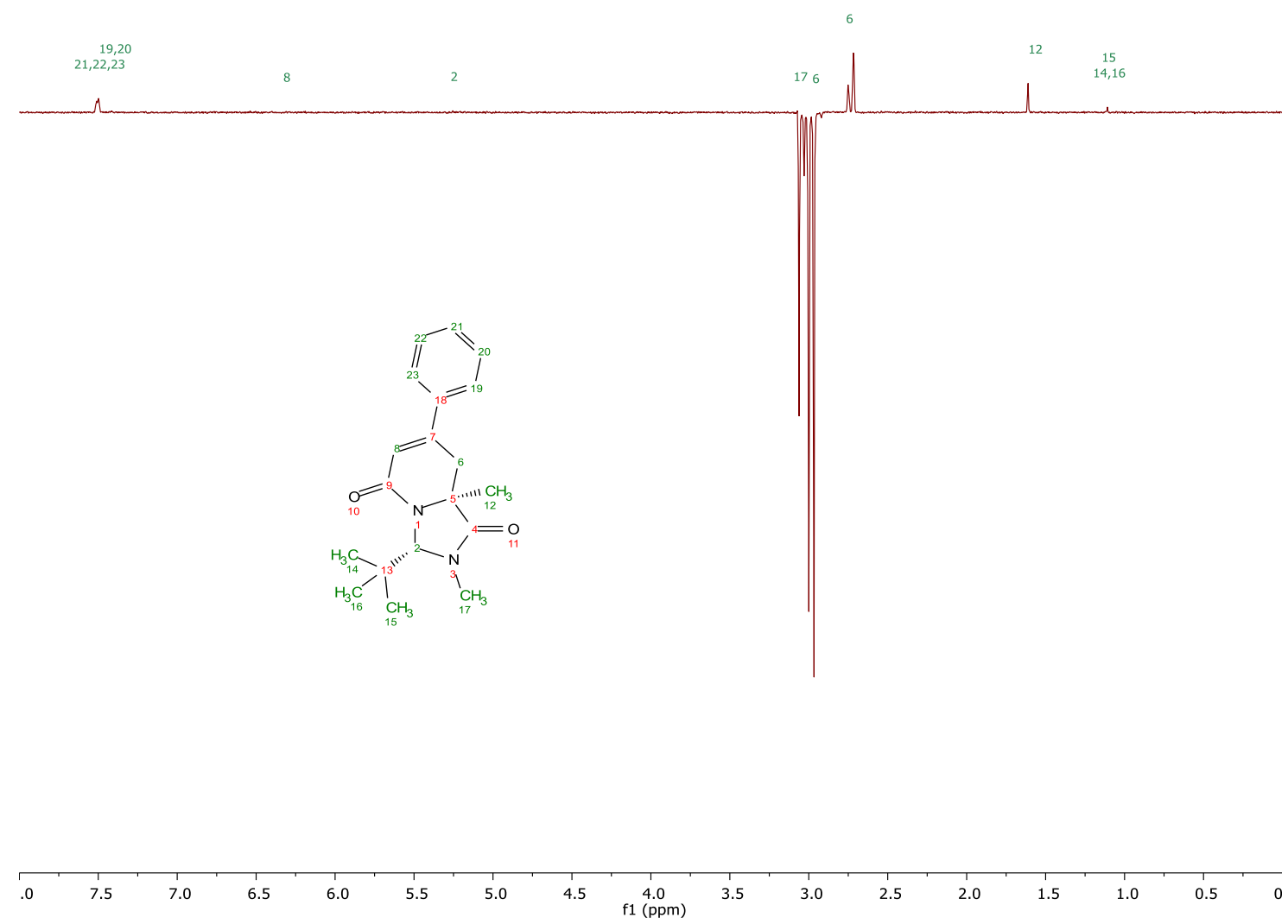




\section{Compound 4b}

JC17336_JC-27ZFF_PROTON01

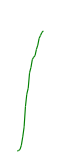<smiles>CC1(C)C=C(c2ccncn2)CC(=O)N1C(C(=O)O)C(=O)O</smiles>

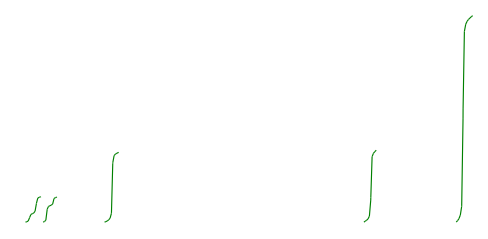

$14,15,16$

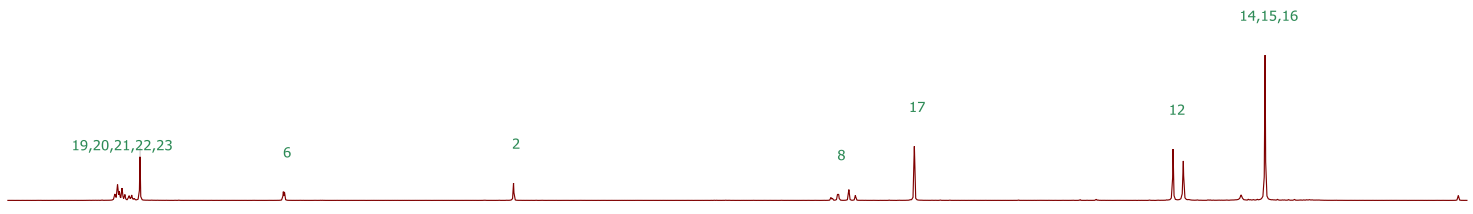

\begin{tabular}{|c|c|c|c|c|c|c|c|c|c|c|c|c|c|c|}
\hline 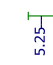 & & 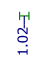 & & & & & & 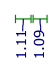 & 岱 & & & & 岱 & \\
\hline 7.5 & 7.0 & 6.5 & 6.0 & 5.5 & 5.0 & 4.5 & $\begin{array}{c}4.0 \\
\mathrm{f} 1(\mathrm{ppm})\end{array}$ & 3.5 & 3.0 & 2.5 & 2.0 & 1.5 & 1.0 & 0.5 \\
\hline
\end{tabular}
JC17336_JC-27-F7_NOESY1D02
6
$14,15,16$

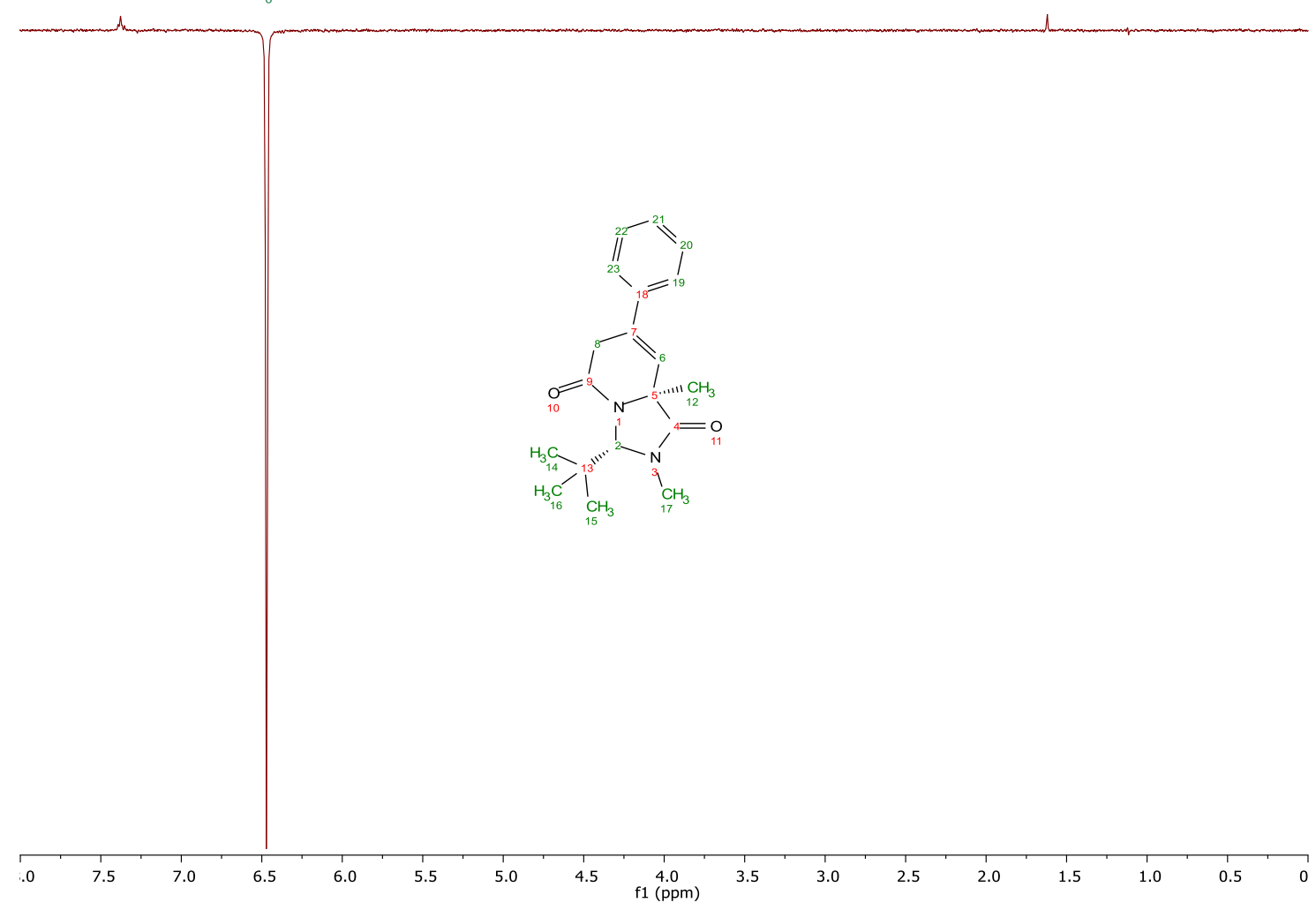




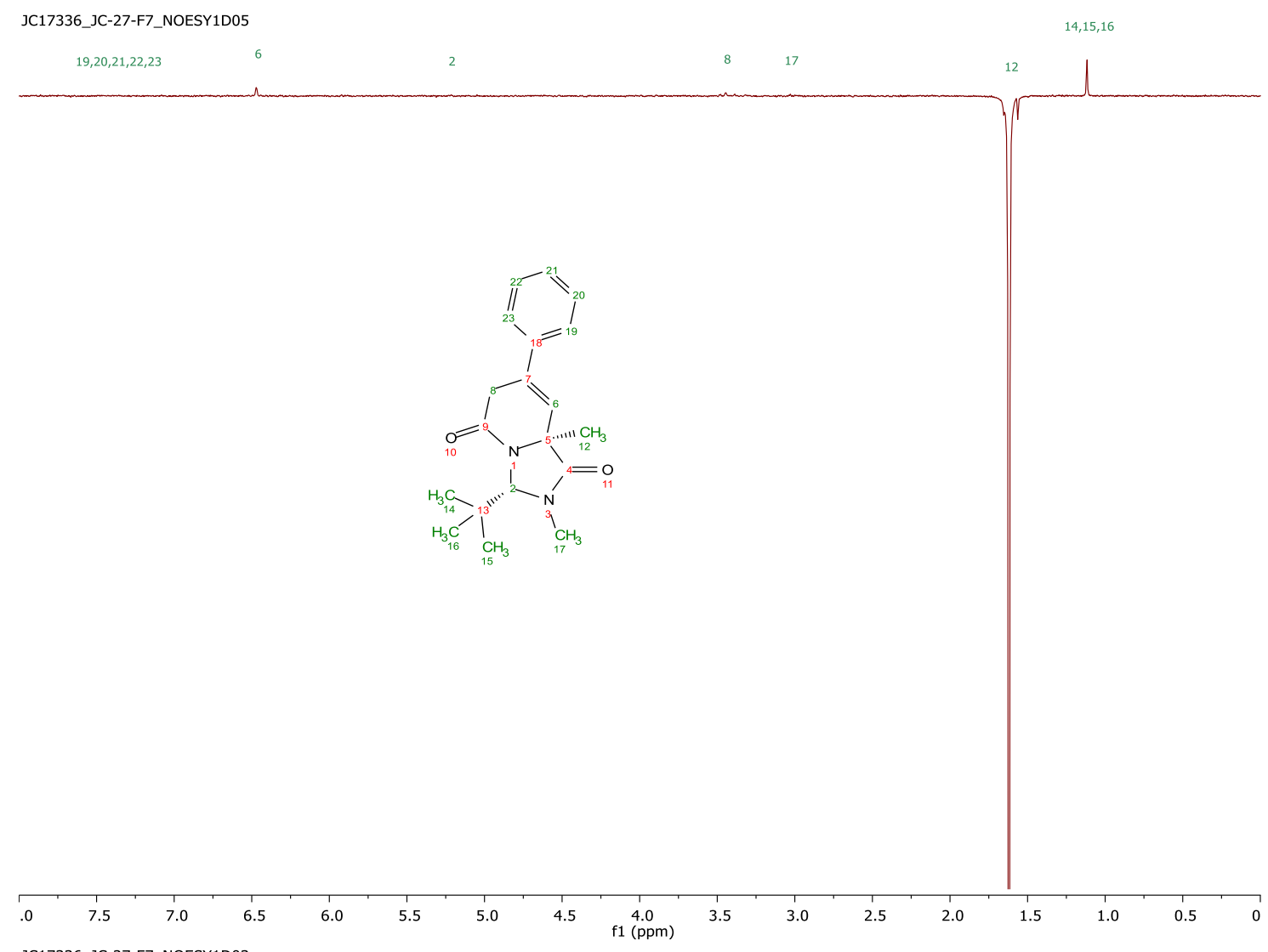
JC17336_JC-27-F7_NOESY1D03

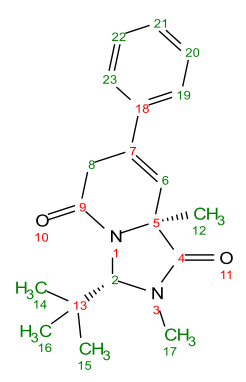

\begin{tabular}{|c|c|c|c|c|c|c|c|c|c|c|c|c|c|c|}
\hline 3.0 & 7.5 & 7.0 & 6.5 & 6.0 & 5.5 & 5.0 & 4.5 & $\begin{array}{r}4.0 \\
\mathrm{f} 1(\mathrm{ppm})\end{array}$ & 3.5 & 3.0 & 2.5 & 2.0 & 1.5 & 1.0 \\
\hline
\end{tabular}




\section{Compound 5}

jc17351_jc-22_PROTON01

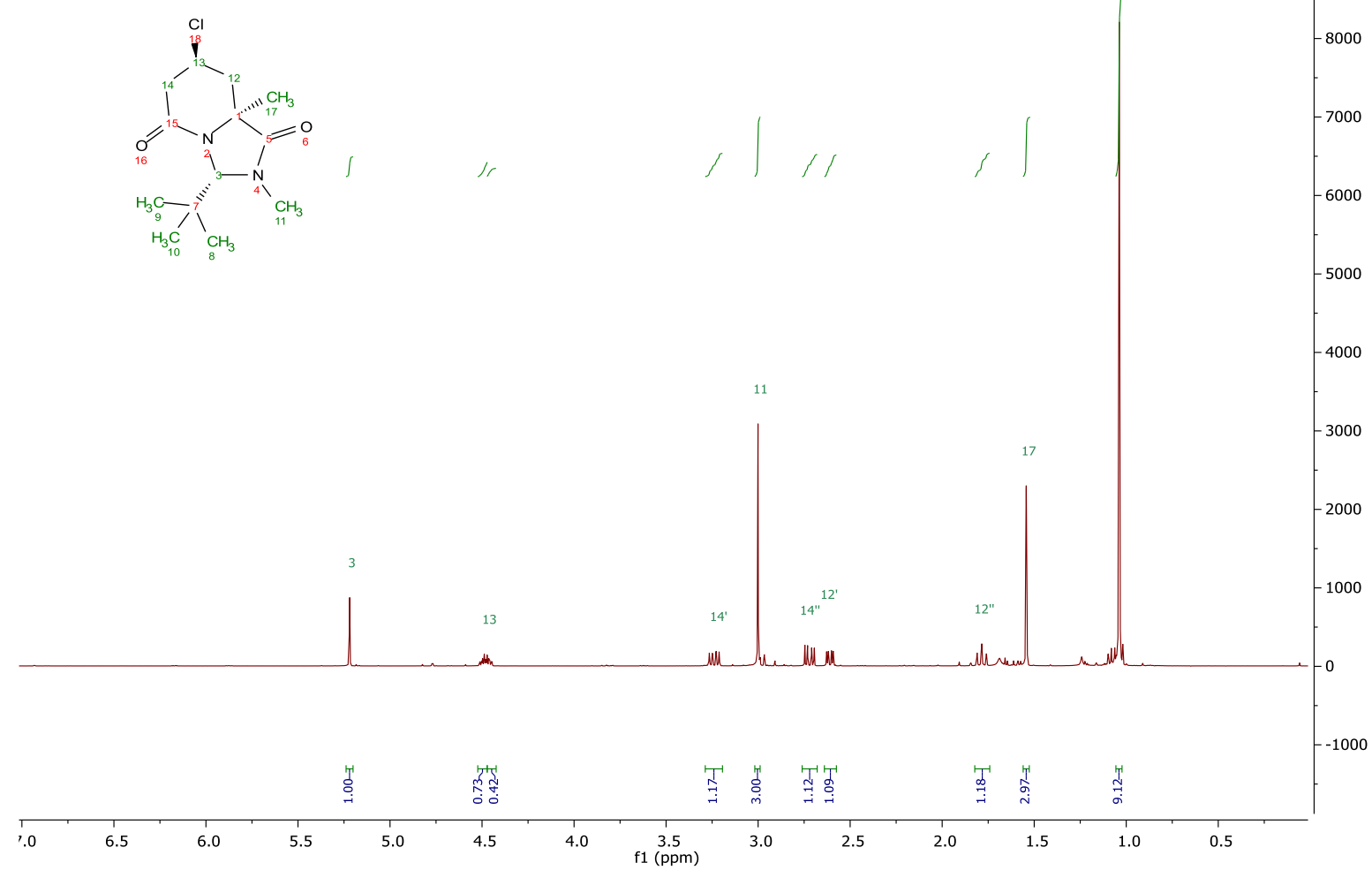

jc17351_jC-22_NOESY1D01

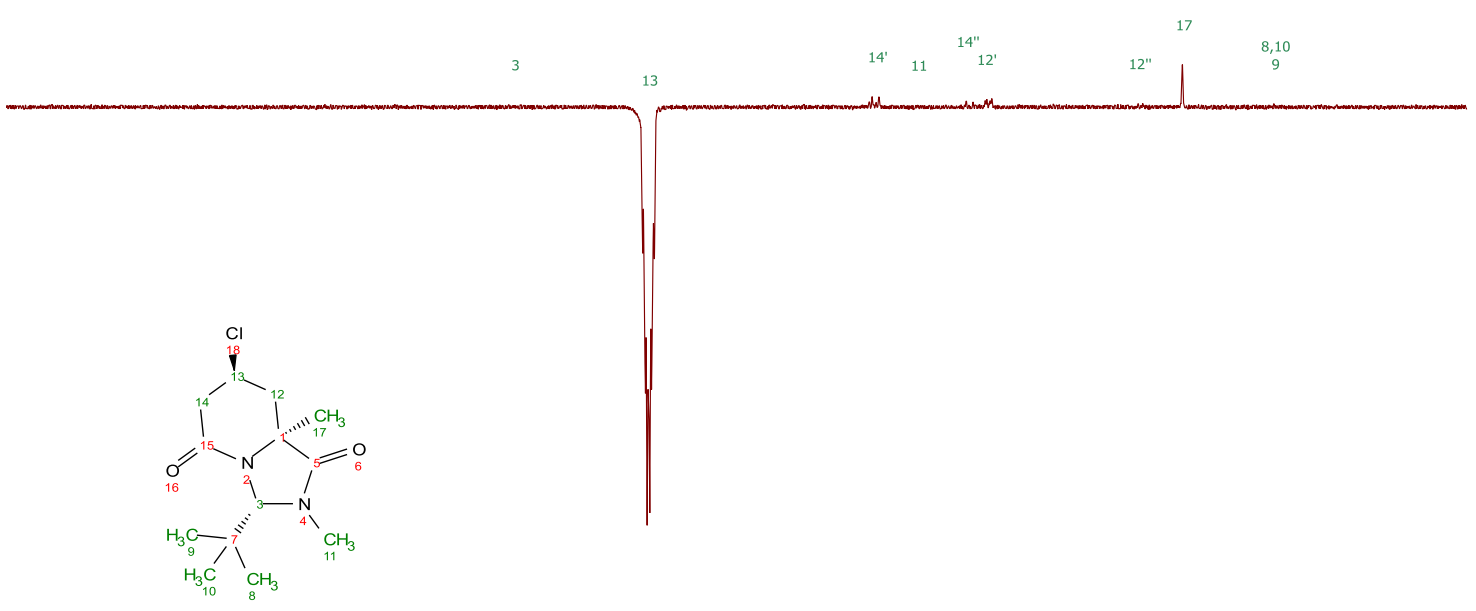

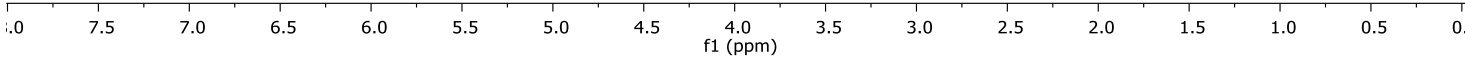




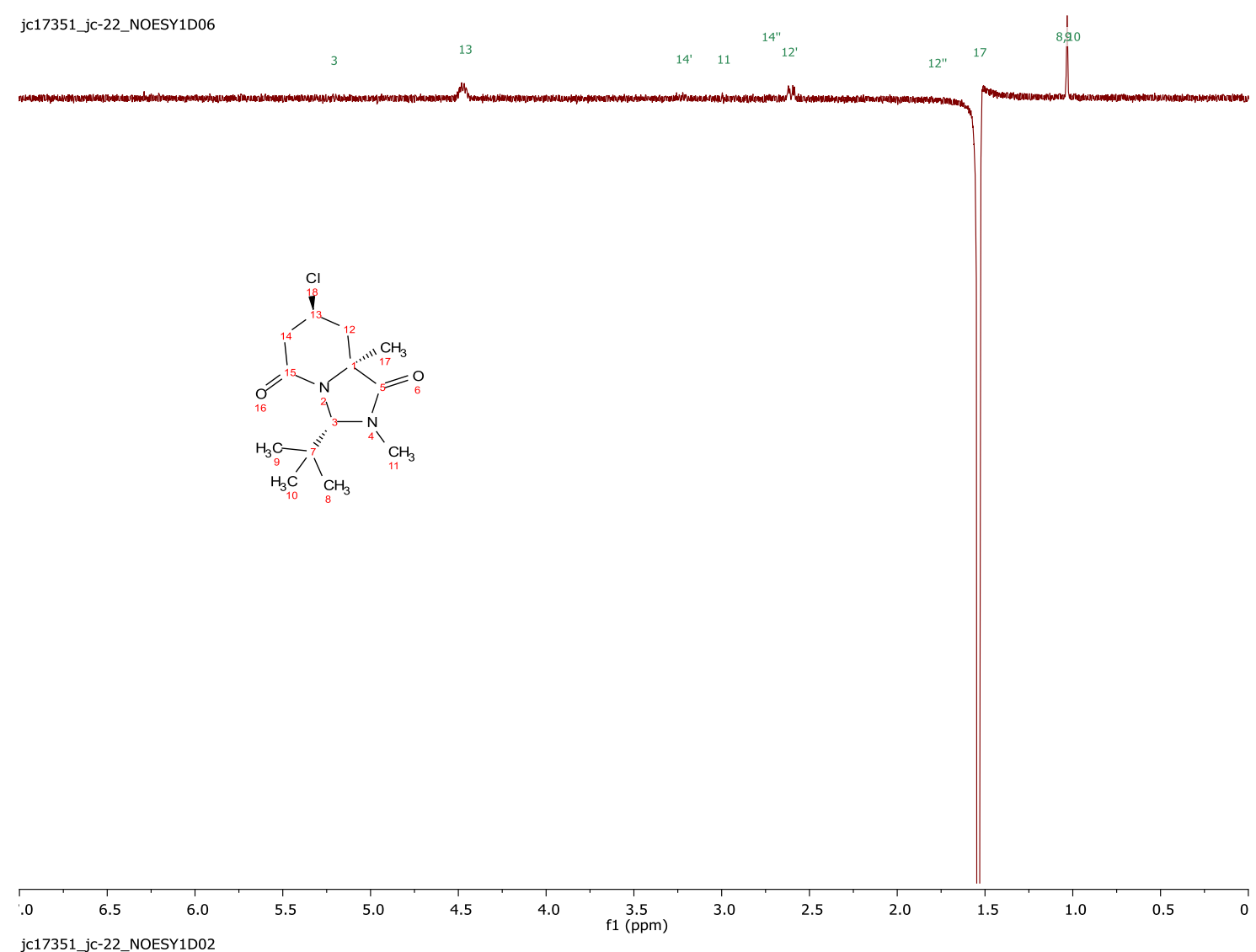
jc17351_jc-22_NOESY1D02

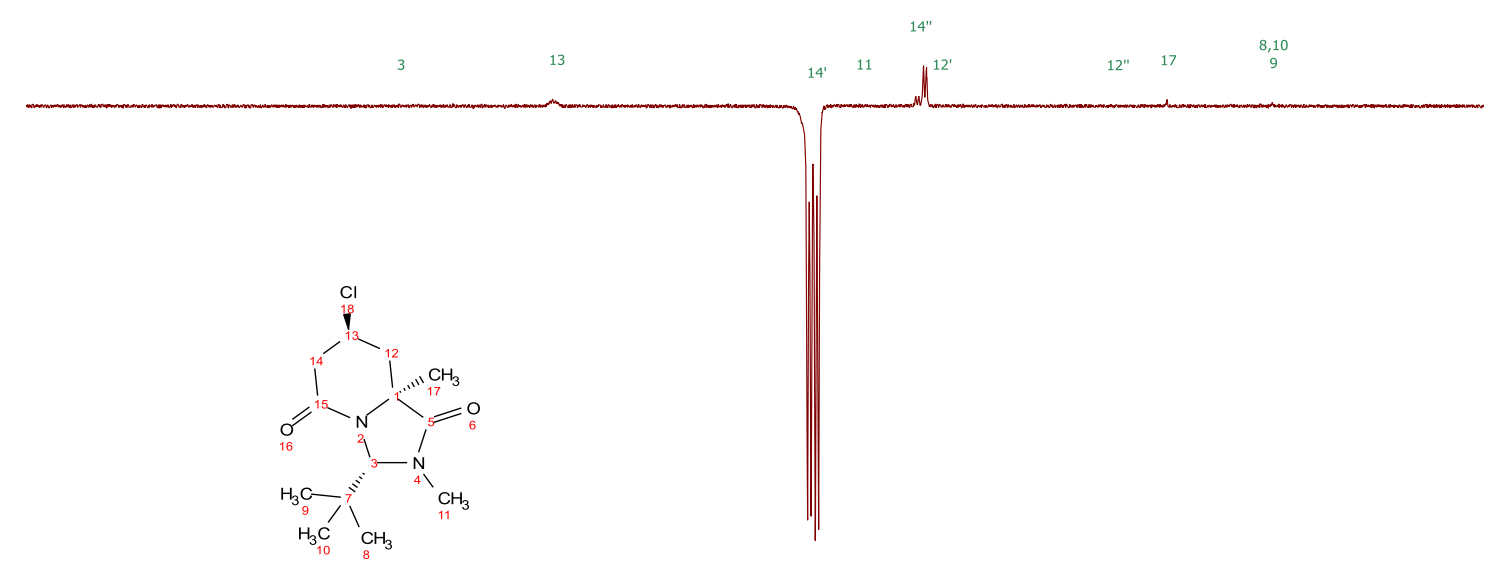

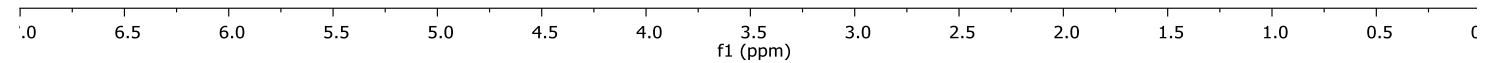



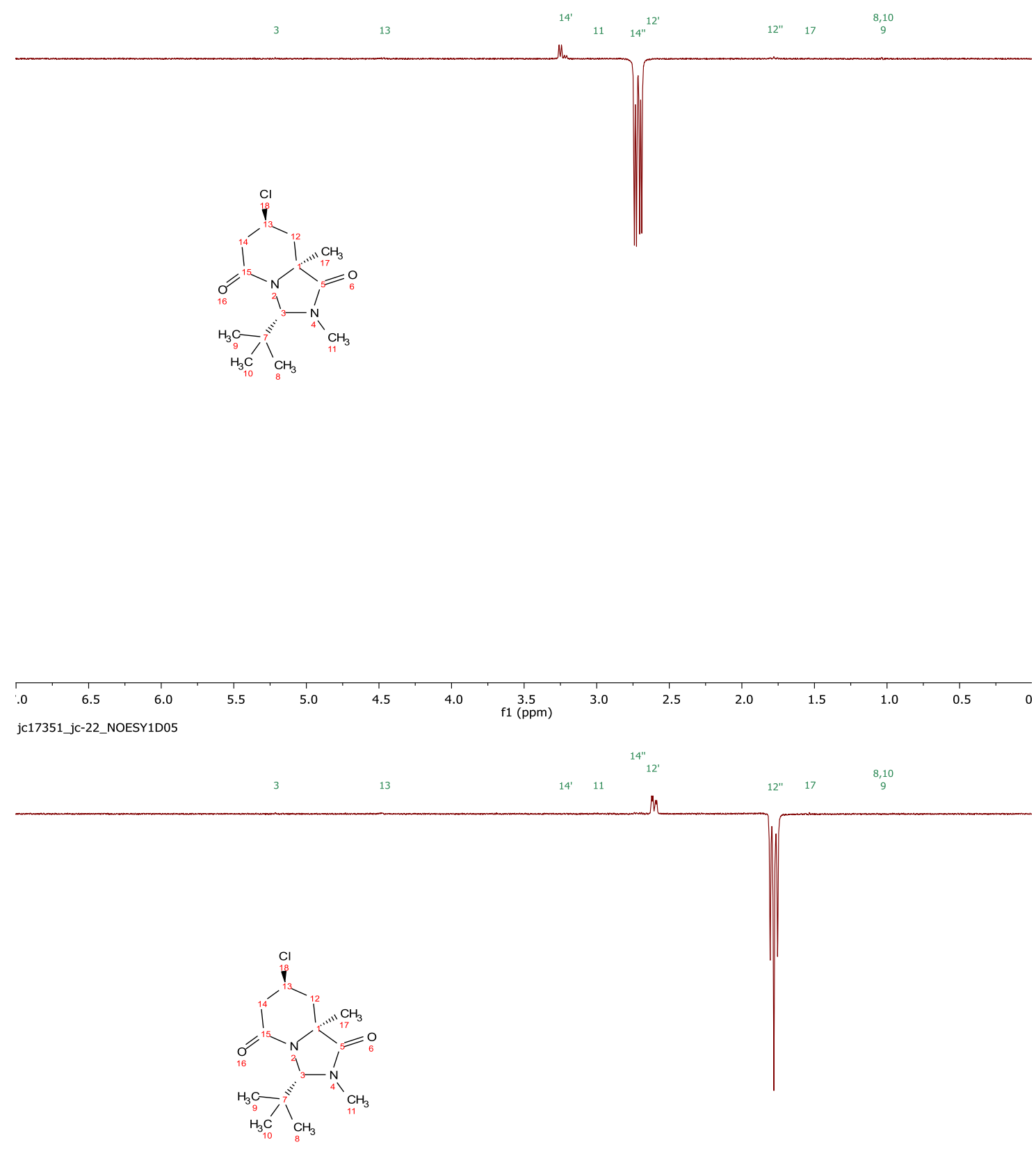

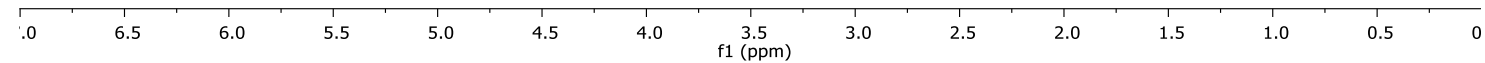



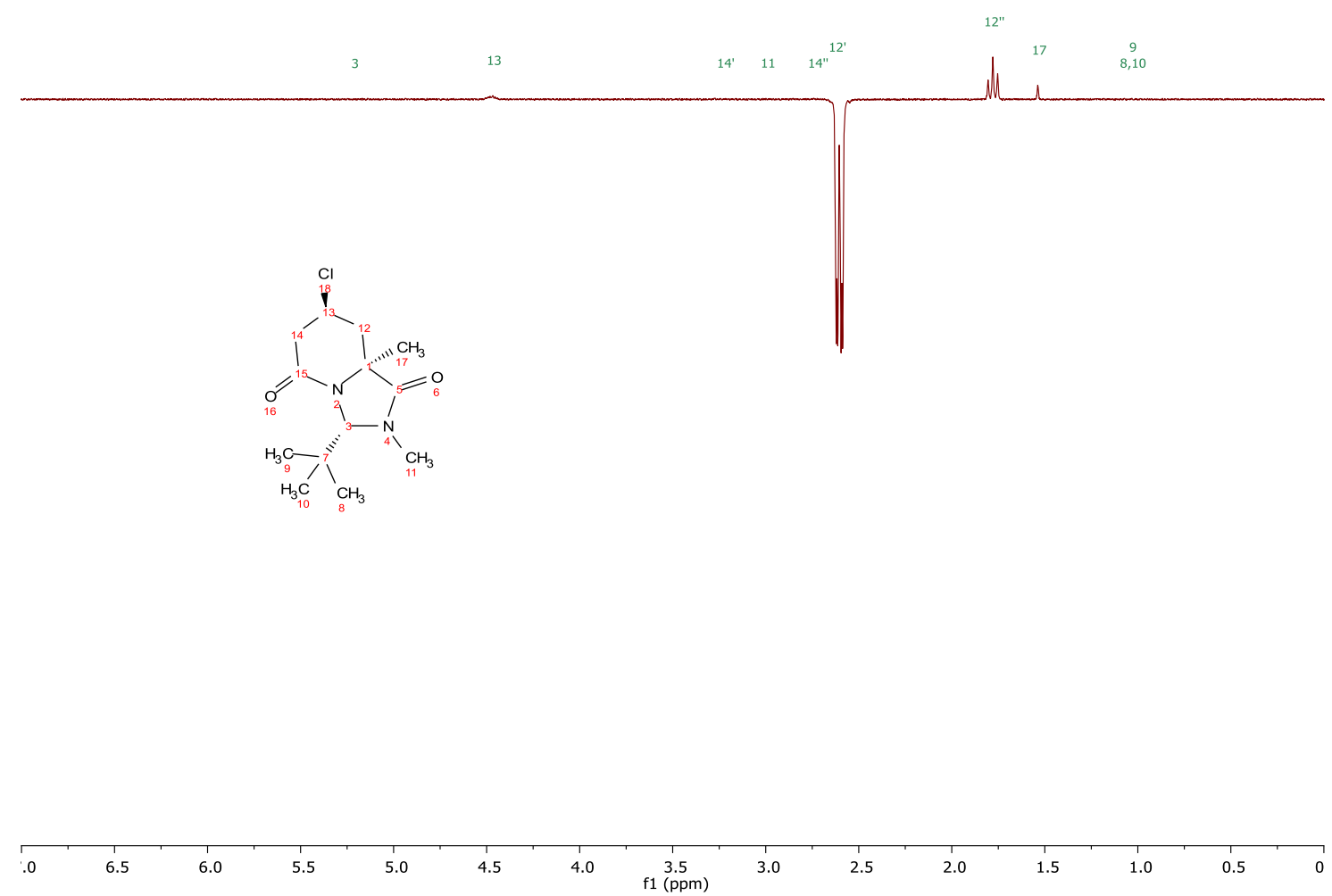

\section{Compound 10a}

$\underset{\substack{\overrightarrow{0} \\ \stackrel{0}{0}}}{\stackrel{\ddot{i}}{0}}$

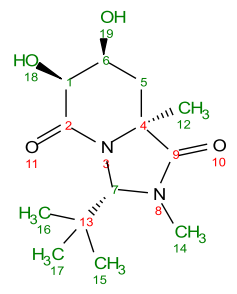

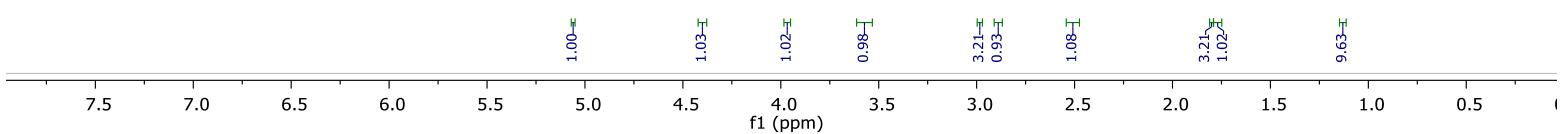




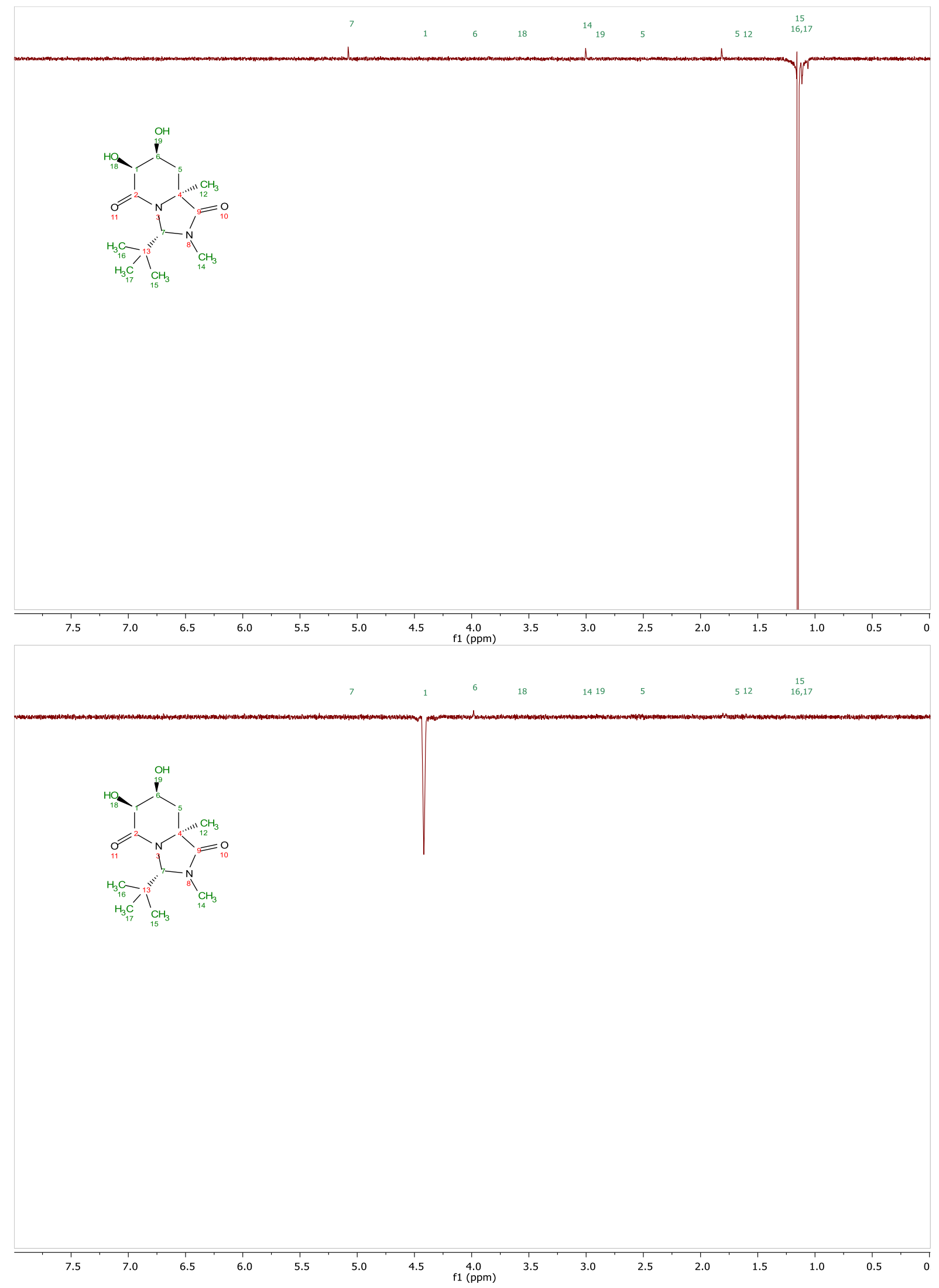




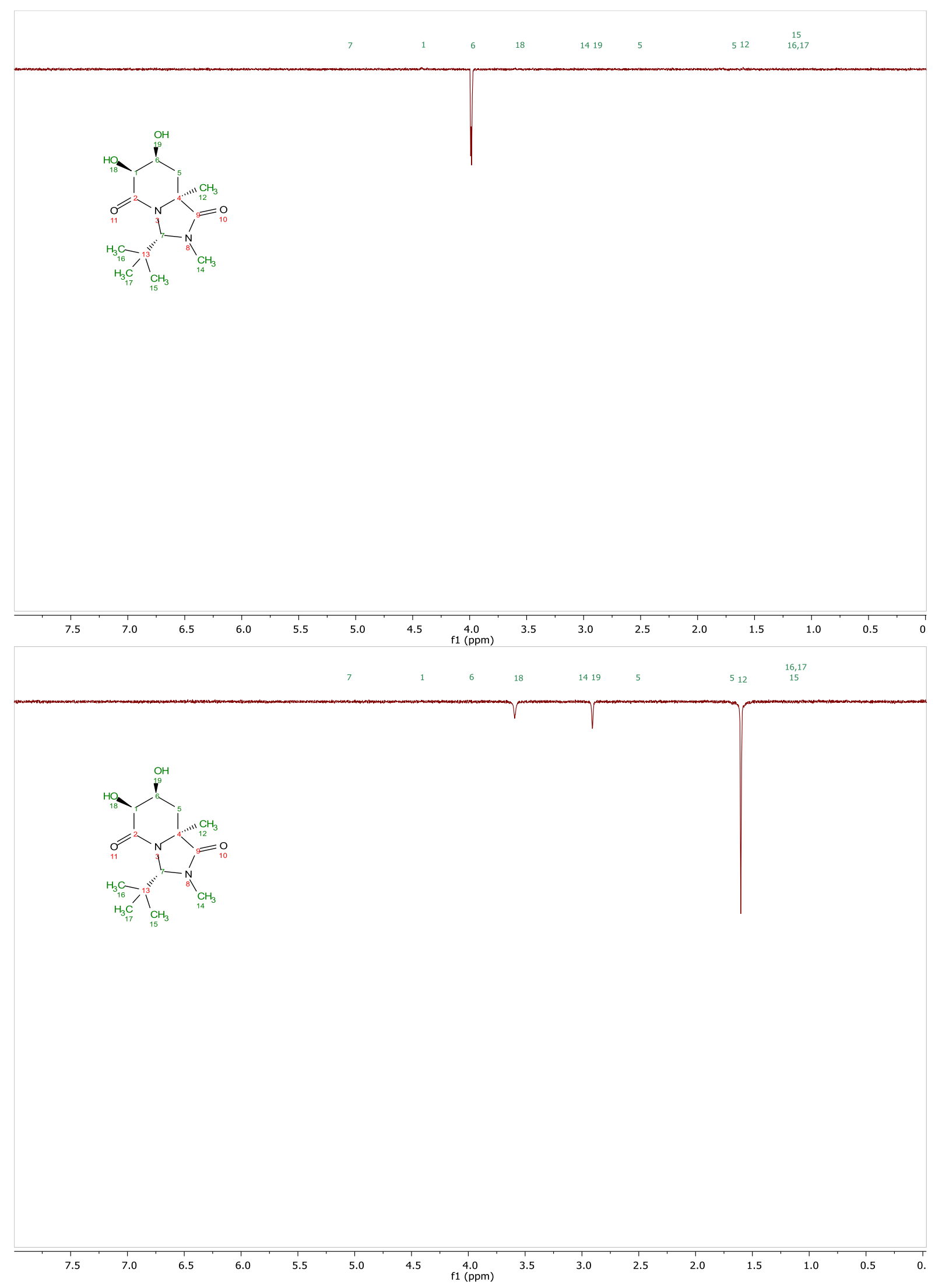




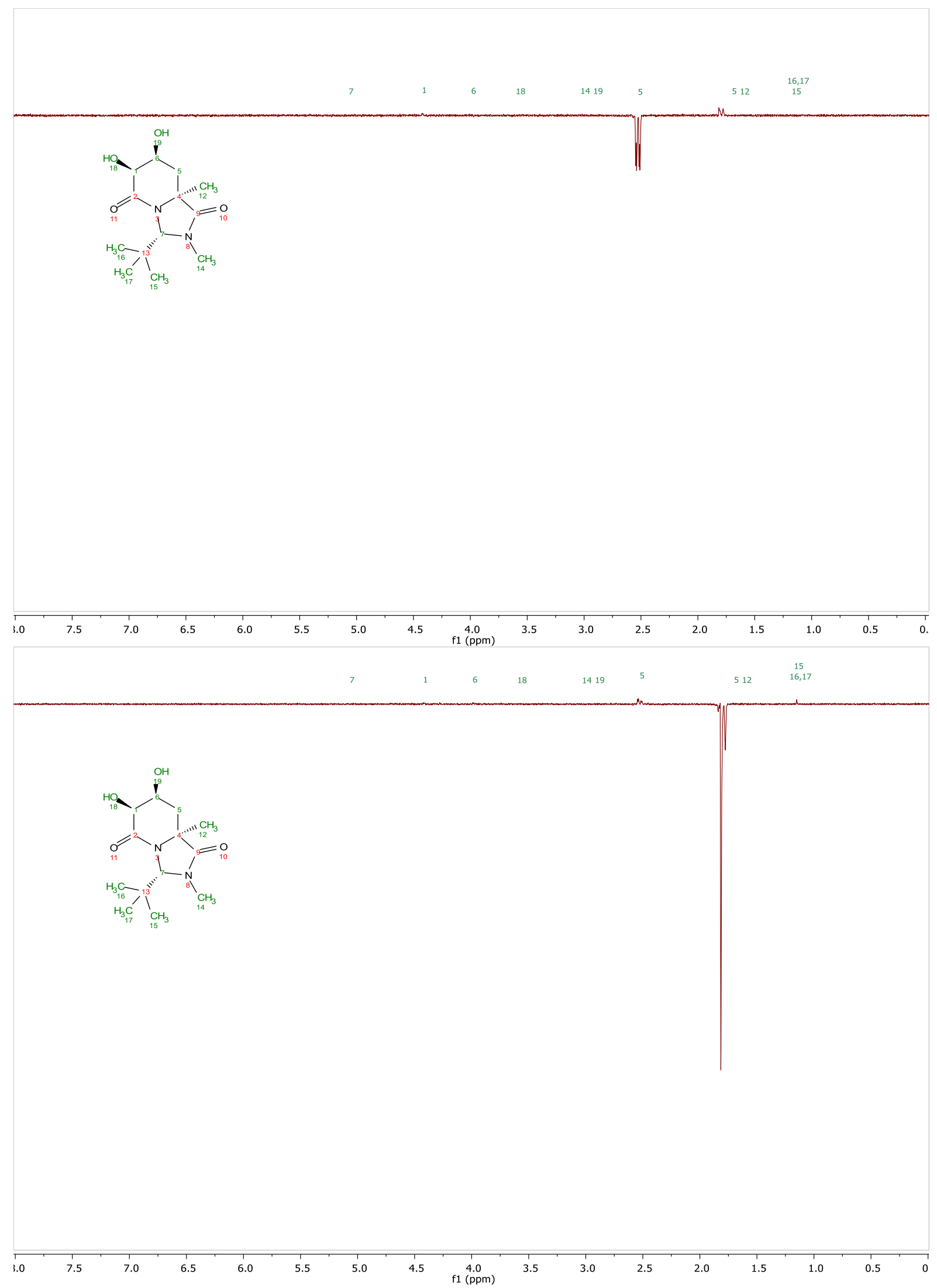




\section{Compound 9a}

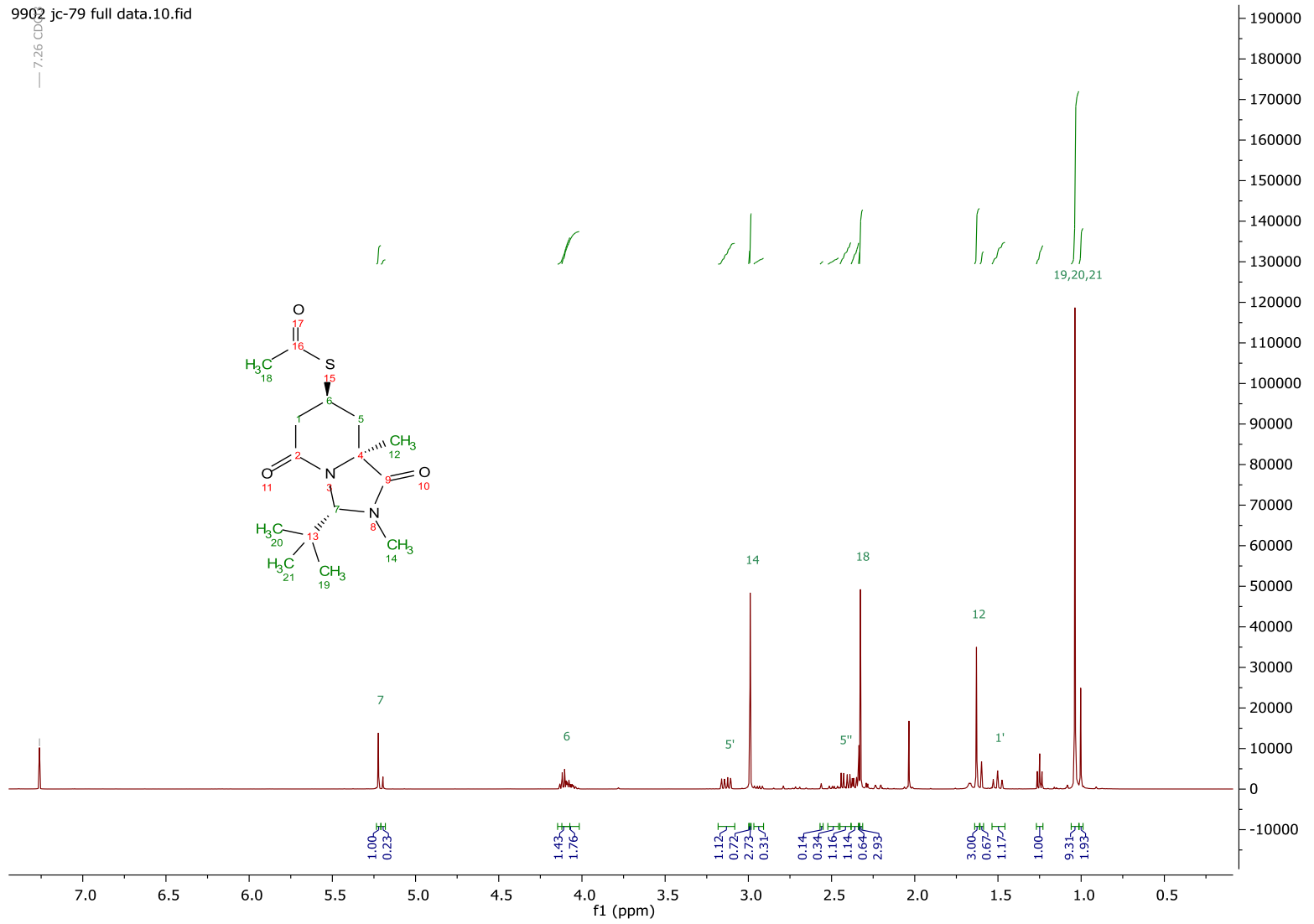

jc22508_jc-79_maddn_NOESY1D_001

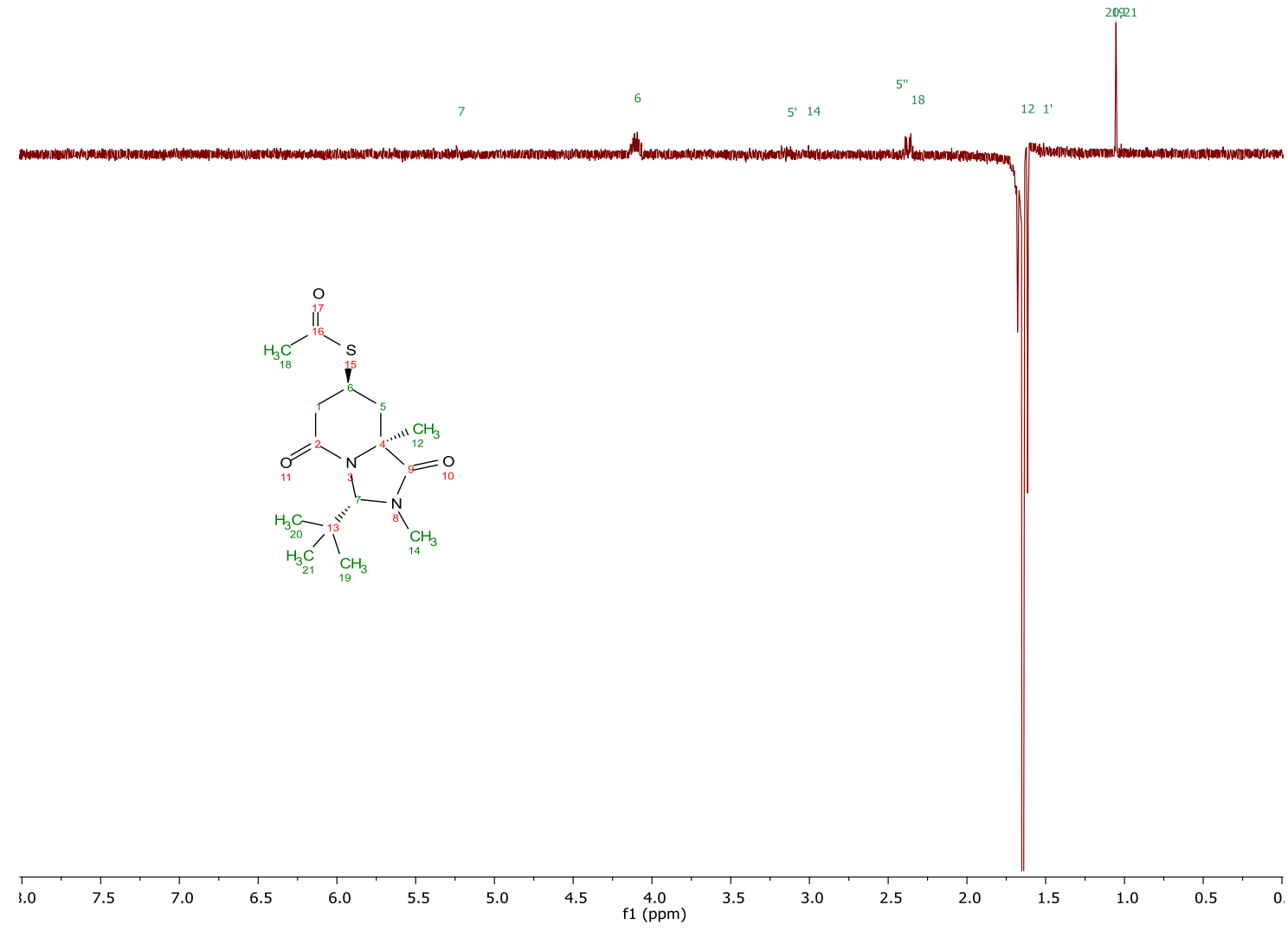




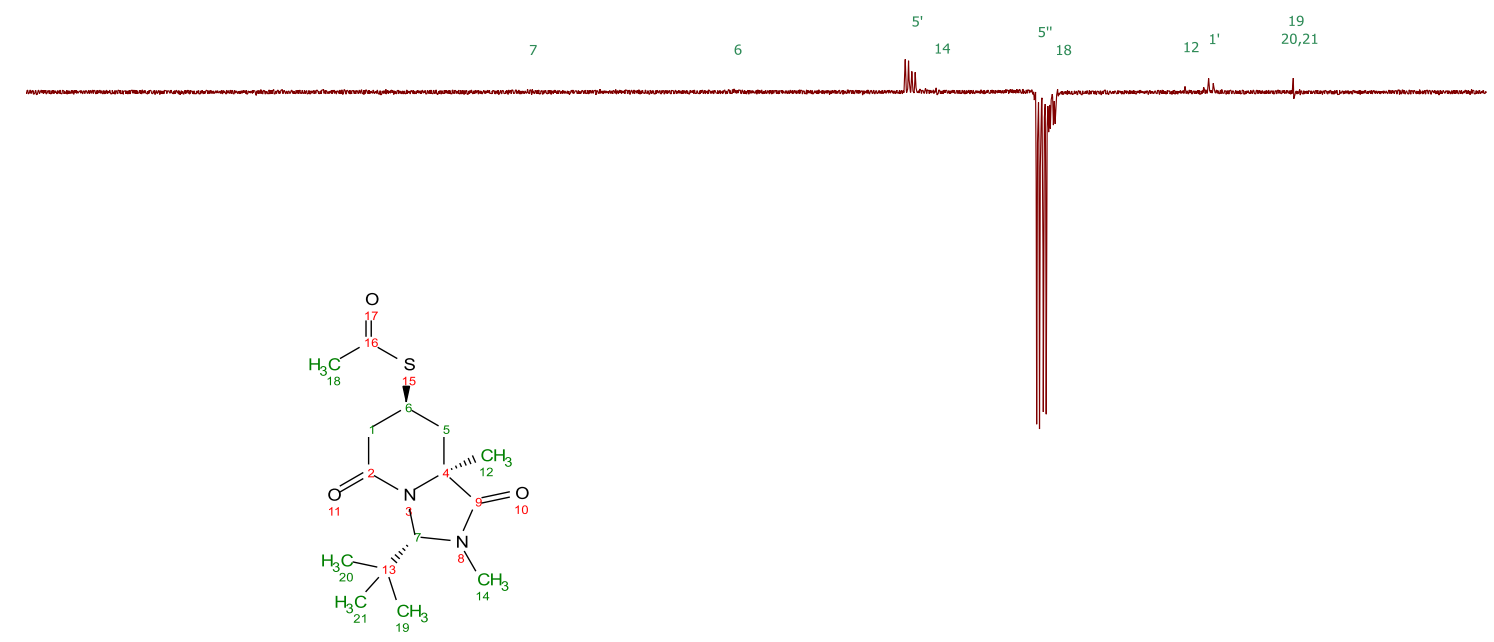

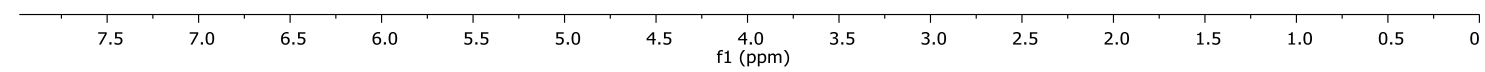

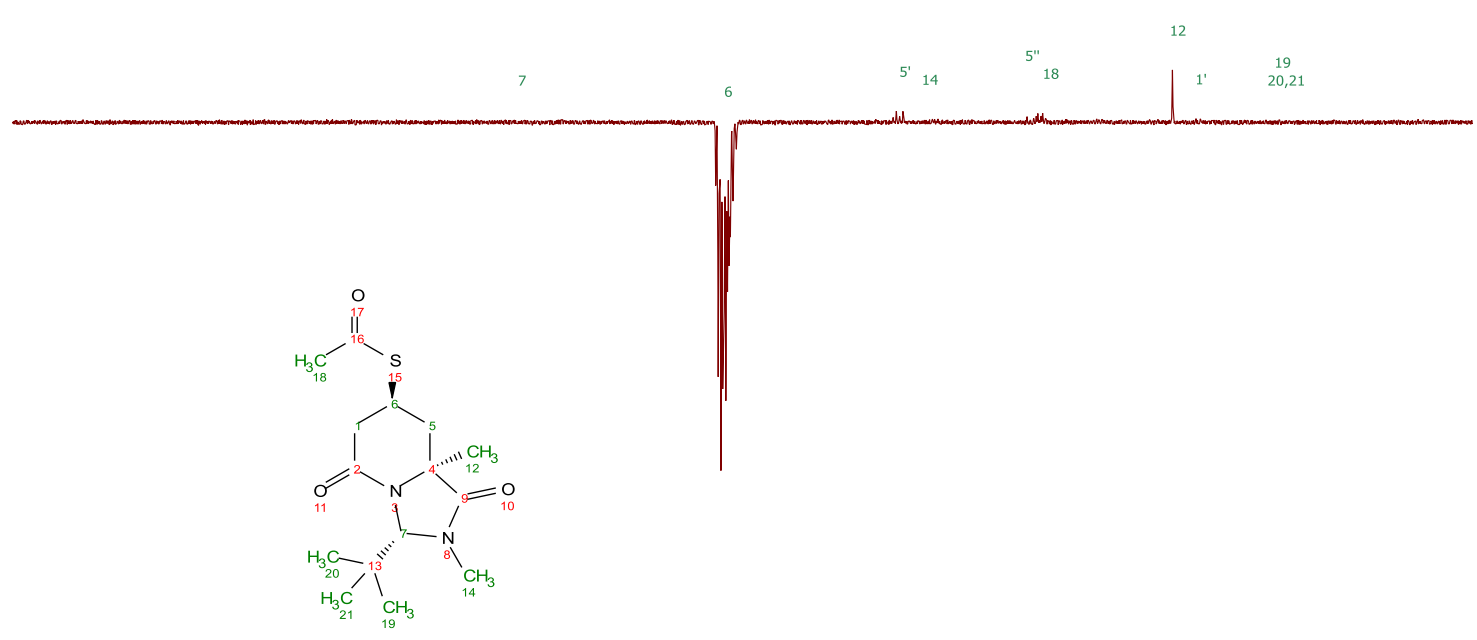

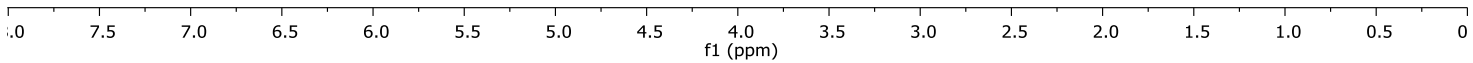




\section{Compound 9b}

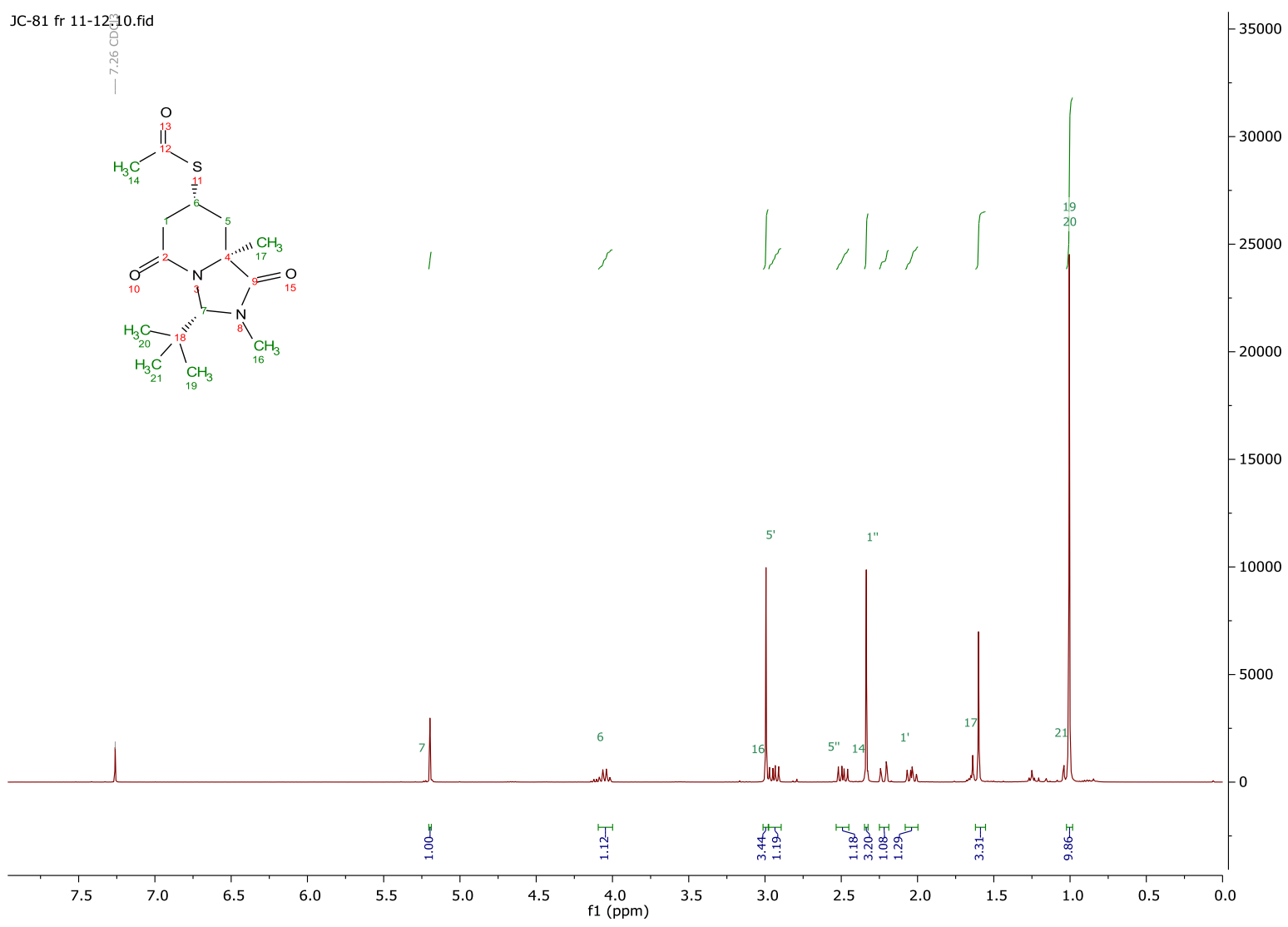




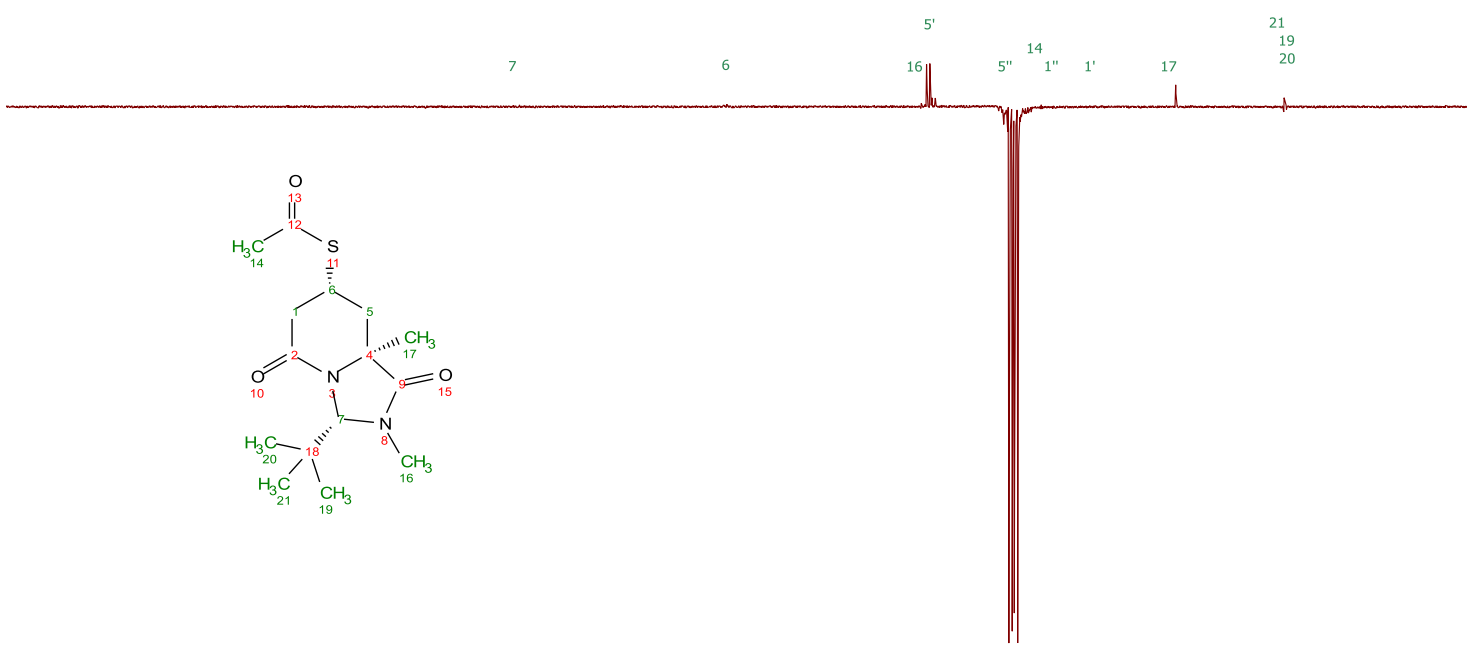

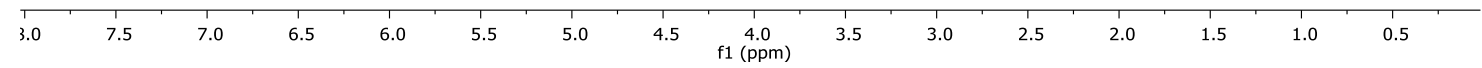
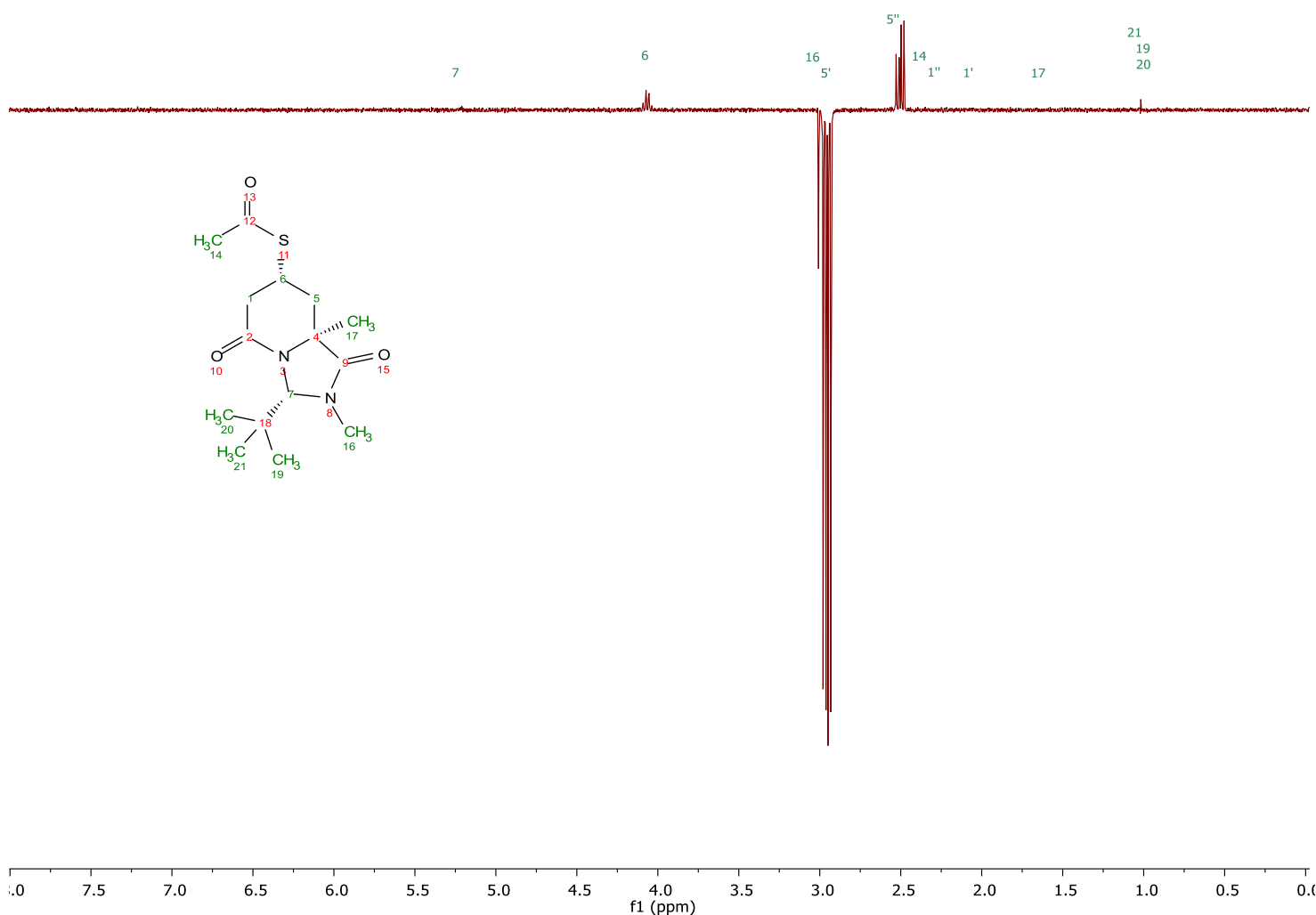

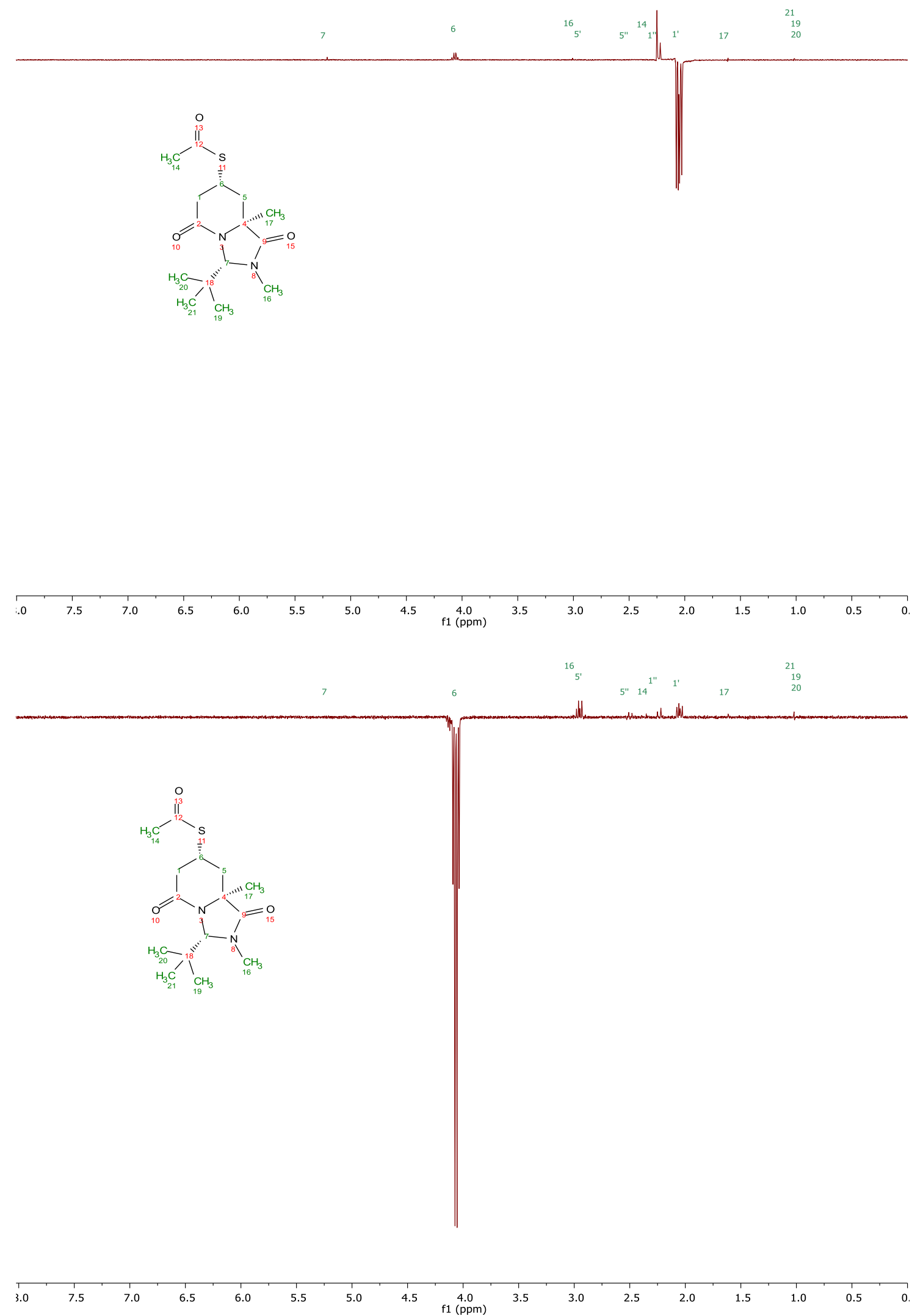


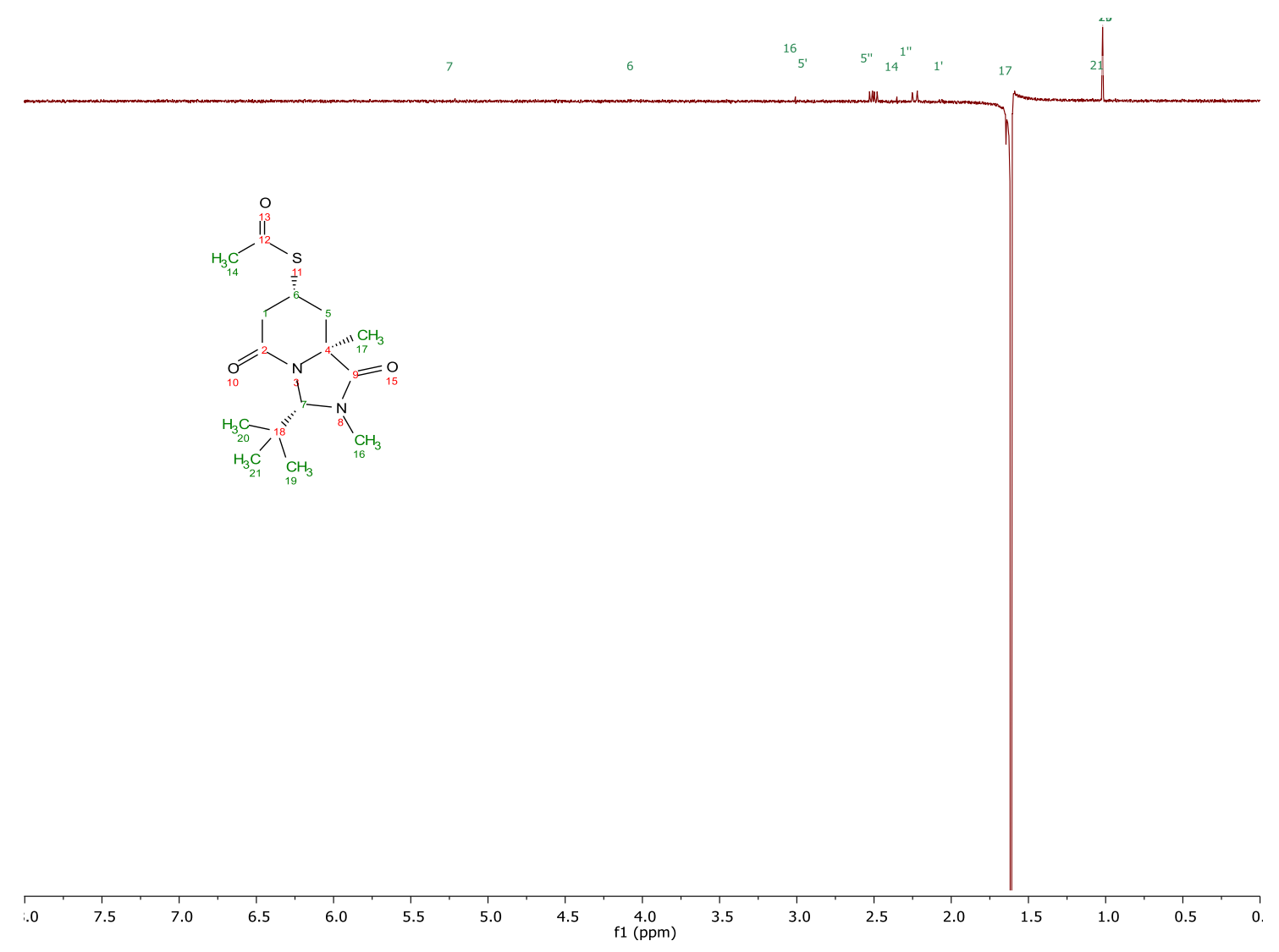

\section{References}

1 D. J. Leonard, J. W. Ward and J. Clayden, Nature, 2018, 562, 105-109.

2 H. Abas, M. M. Amer, O. Olaizola and J. Clayden, Org. Lett., 2019, 21, 1908-1911.

3 Isis Innovation Limited, 20070142632, 2005.

4 D. J. Vyas and M. Oestreich, Chem. Commun., 2010, 46, 568-570.

5 T. J. Donohoe, L. P. Fishlock and P. A. Procopiou, Org. Lett., 2008, 10, 285-288.

6 A. Cabré, G. Sciortino, G. Ujaque, X. Verdaguer, A. Lledós and A. Riera, Org. Lett., 2018, 20, 5747-5751.

7 Q. J. Liang, C. Yang, F. F. Meng, B. Jiang, Y. H. Xu and T. P. Loh, Angew. Chemie - Int. Ed., 2017, 56, 5091-5095.

8 W. R. Roush and B. B. Brown, J. Org. Chem., 1992, 57, 3380-3387. 